\title{
OPERATIONAL OPTIMIZATION OF RESIDENTIAL HVAC SYSTEM USING MODEL PREDICTIVE CONTROL STRATEGY PLANNING
}

\author{
by
}

\author{
Nima Alibabaei \\ Master of Science in Operating Research, \\ Tarbiat Modares University, Iran, 2008 \\ Bachelor of Engineering in Power Systems, \\ Tehran University, Iran, 2003 \\ A dissertation \\ presented to Ryerson University \\ in partial fulfillment of the \\ requirements for the degree of \\ Doctor of Philosophy \\ in the Program of \\ Mechanical and Industrial Engineering
}

Toronto, Ontario, Canada, 2017

(C) Nima Alibabaei, 2017 


\section{Author's Declaration}

I hereby declare that I am the sole author of this dissertation. This is a true copy of the dissertation, including any required final revisions, as accepted by my examiners.

I authorize Ryerson University to lend this dissertation to other institutions or individuals for the purpose of scholarly research.

I further authorize Ryerson University to reproduce this dissertation by photocopying or by other means, in total or in part, at the request of other institutions or individuals for the purpose of scholarly research.

I understand that my dissertation may be made electronically available to the public. 


\section{Acknowledgements}

I would like to thank my supervisor, Dr. Alan S. Fung, for providing me with the opportunity to conduct Ph.D. level research in Canada. His continuous guidance along with his profound technical insight kept me focused on the problem at hand during the course of my research. He also provided me with abundant opportunities to write the internship/assistantship proposals and technical journal and conference articles and prepare posters. In addition, I would like to thank him for his immense assistance in employing and field-testing the developed controller in the TRCA Archetype Sustainable House (ASH). I am thankful to Dr. Kaamran Raahemifar for cosupervising me by providing feedback during my research and allocating significant time to review my research. I also thank him for allotting me valuable human resources such as visiting students and connecting me to technical and professional resources. Dr. Fung and Dr. Raahemifar supported me in my fight with brain cancer, for which I am grateful to them both.

Special thanks go to Mr. David Nixon and Mr. Gil Amdurski of TRCA for their help during the implementation of the HVAC system fuel-switching controller. Without their help, the implementation of this project would not have been possible.

I am also thankful to my friends at Ryerson University, Abdul Afram, Danilo Yu, Arash Moghimi, and all the others that I forgot to mention for their cooperation and making my time enjoyable. I have spent quite a few memorable moments with them and made long-term friends during the course of my Ph.D. study.

This research was financially supported by the Natural Sciences and Engineering Research Council (NSERC) of Canada, Smart Net-Zero Energy Buildings Research Network (SNEBRN), and the Toronto Hydro and Mitacs-Accelerate Program. The resources such as the HVAC system, monitoring system, and data were provided by the Toronto and Region Conservation Authority (TRCA). I am also thankful to Toronto Hydro for internship assistance, which helped me both from financial and research points of view. I am thankful to Ryerson University for providing me with several awards to complete my Ph.D. study. These awards included the Mechanical Engineering Graduate Award, the Mechanical Engineering Graduate Scholarship, and teaching assistantships (TA) in various courses. 


\section{Dedication}

To my family including my wife Sara, who supported me in my fight with brain cancer, for which I am grateful to her, mother, father, brother, sister and my uncle Parviz Saadat. Without their continued love, assistance, encouragement and confidence I may not have succeeded in this journey. 


\section{Abstract \\ OPERATIONAL OPTIMIZATION OF RESIDENTIAL HVAC SYSTEM USING MODEL PREDICTIVE CONTROL STRATEGY PLANNING MODEL}

Doctor of Philosophy, 2017

Nima Alibabaei

Department of Mechanical and Industrial Engineering, Ryerson University

To date, the residential sector accounts for a major portion of consumption by consuming more than $40 \%$ of the entire world's energy and producing $33 \%$ of the carbon dioxide emissions. In North America, the residential sector energy consumptions are mainly related to heating, ventilation, and air conditioning (HVAC) systems, which are not operating in the most efficient ways due to existing on/off and conventional controllers. In Ontario, due to the variable price of electricity, variation in outdoor disturbances, and new Ontario Government sweeping mandate in overhauling the energy use in residential sector, there is an opportunity to develop intelligent control systems to employ energy conservation strategy planning model (ECSPM) in existing HVAC systems for reducing their operating cost, energy consumption, and GHG emission.

In order to take advantage of these opportunities, two model-based predictive controllers (MPCs) were developed in this Ph.D. research. In the first MPC controller, a Matlab-TRNSYS co-simulator was developed to fill the lack of advanced controllers in building energy simulators. This cosimulator investigated the effectiveness of different novel ECSPMs on an HVAC system's energy cost saving during winter and summer seasons. This co-simulator offered $23.8 \%$ saving in the HVAC system's energy costs in the heating season. Regardless of the strong capabilities, employing this co-simulator for implementing comprehensive/complex optimization methods resulted in an unacceptably long optimization time due to the of TRNSYS simulation engine. Therefore, in the second PMC controller, simplified house thermal and HVAC system models were developed in Matlab. To design a grid-friendly house, this model was enhanced by integrating on-site renewable energy generation and storage systems. A novel algorithm was developed to reduce the MPC controller optimization time. The effectiveness of the novel MPC model in the HVAC system's energy cost saving was compared with a Simple Rule-based (SRB) controller, which itself is an efficient HVAC controller, while this controller offered $12.28 \%$ additional savings in the heating season. 
Table of Contents

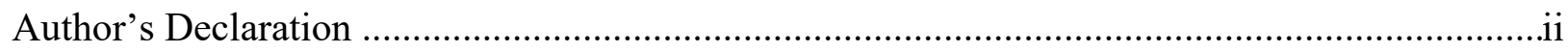

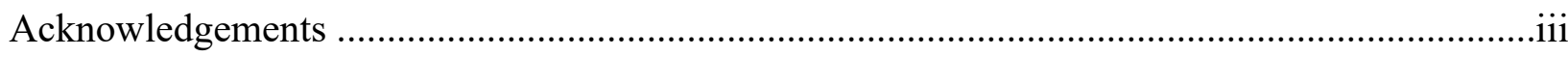

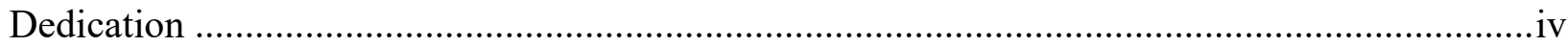

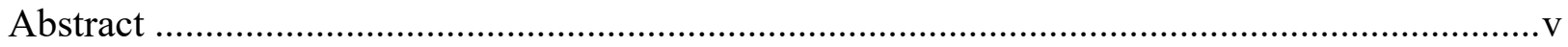

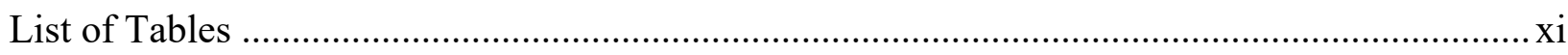

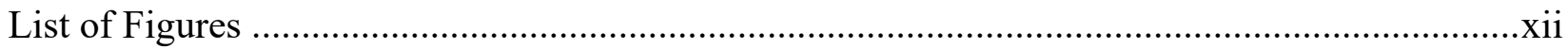

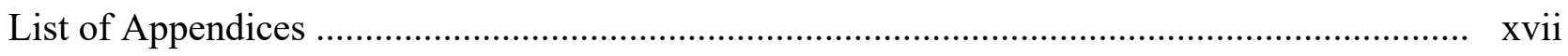

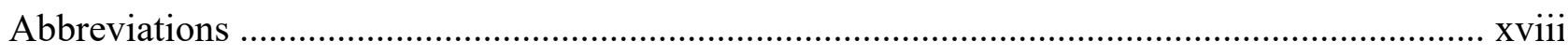

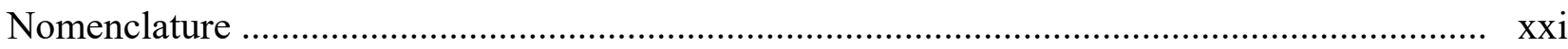

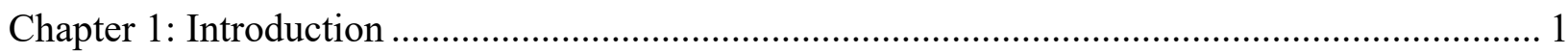

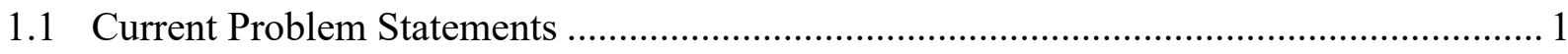

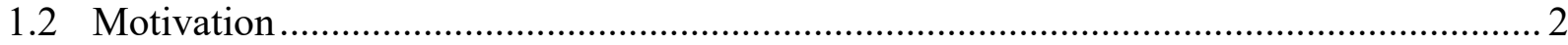

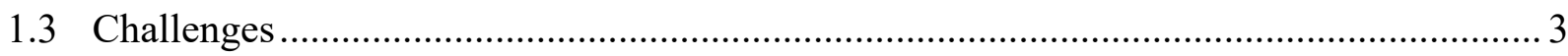

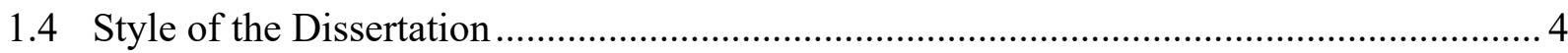

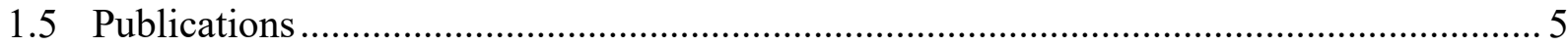

1.6 Structure of the Dissertation ................................................................................. 5

Chapter 2: Review of Control Methods and Energy Conservation Strategy Planning Models in Residential Houses and HVAC Systems ......................................................... 7

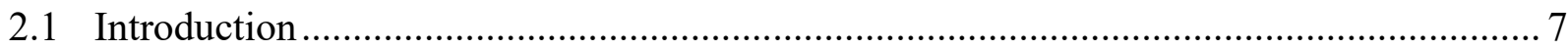

2.2 House and HVAC System Control Approaches ….................................................... 9

2.2.1 Articles Reviewed the HVAC Control Techniques .............................................. 9

2.2.2 Classification of Process Control Methods ............................................................ 10

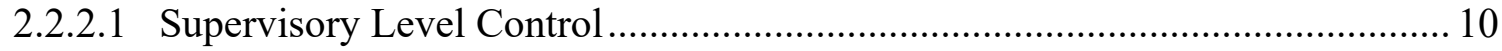

2.2.2.2 Local Level Controls for HVAC System...................................................... 10

2.2.2.3 Classical/Conventional Control .................................................................. 10

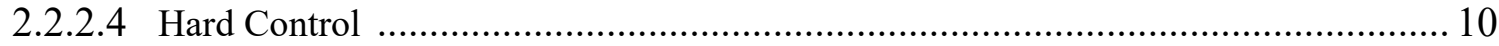

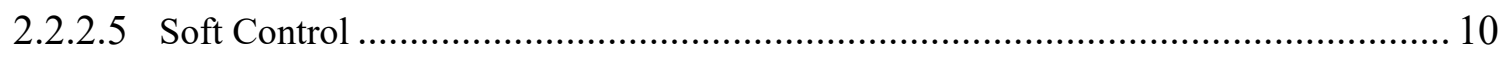

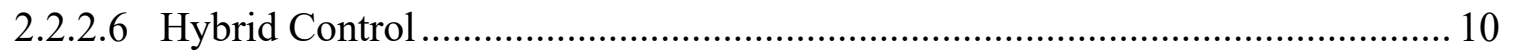


2.2.2.7 Other Control Techniques............................................................................. 10

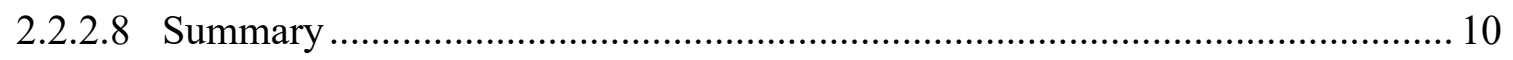

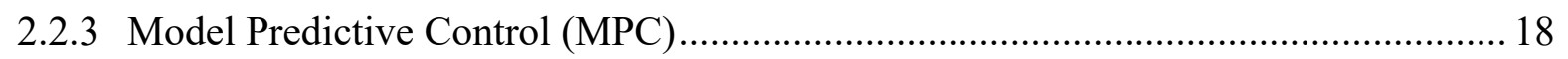

2.2.3.1 Comparing MPC with other Control Technique and Approaches...................... 21

2.2.3.2 Comparing Various MPC Methods and Approaches........................................... 23

2.3 HVAC System Energy Conservation Strategy Planning Models ......................................30

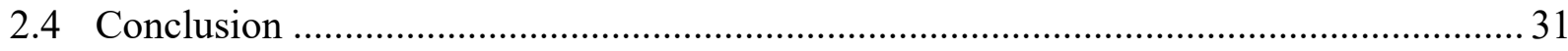

Chapter 3: Development of First MPC Controller Using Matlab-TRNSYS Co-simulator for Applying Predictive Strategy Planning Models ........................................................................... 33

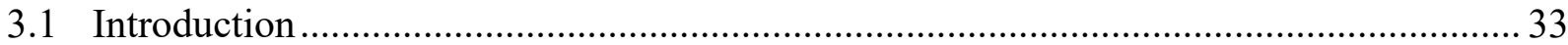

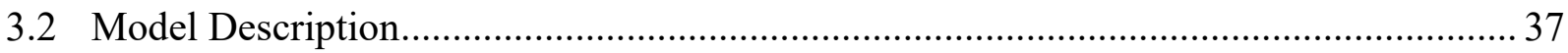

3.2.1 Historical and Forecast Weather Information ................................................................. 37

3.2.2 Estimation of 24 Hours-ahead HVAC System Electrical Demand Based on Weather

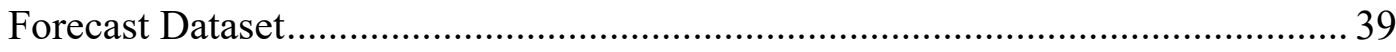

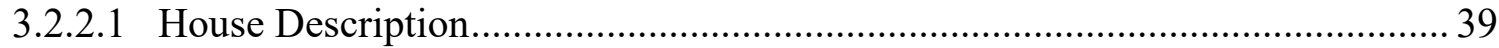

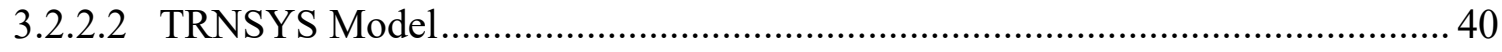

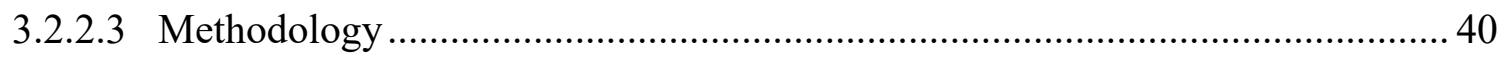

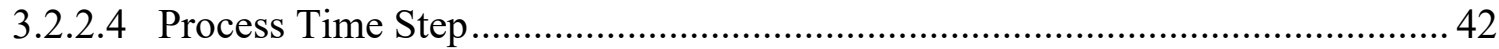

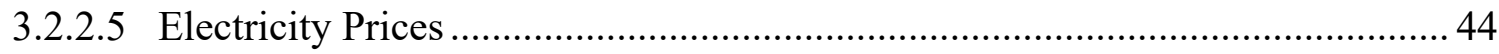

3.2.3 HVAC System Energy Cost Reduction with Smart Dual Fuel Switching System Strategy

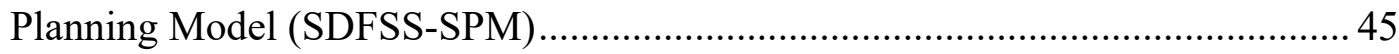

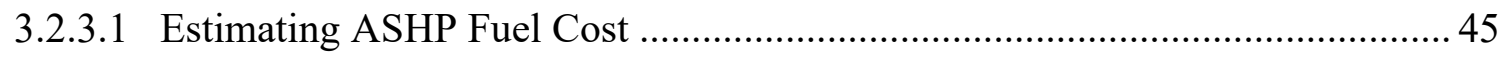

3.2.3.2 Estimating Mini Boiler Fuel Cost ................................................................... 46

3.2.3.3 Using SDFS System as a Strategy Planning Model........................................... 47

3.2.3.4 Calculating the Impact of SDFSS-SPM on HVAC System Daily Fuel Cost (DFC) at Different Outdoor Temperatures

3.2.4 HVAC System Demand Management Using Load Shifting Strategy Planning Model

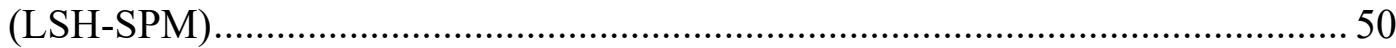

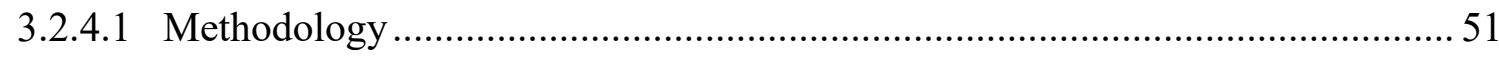

3.2.4.2 Pre-heating Starting Time (Winter Operation) ………………………............ 51

3.2.4.3 Calculating the Impact of LSH-SPM on HVAC System Daily Electricity Cost (DEC) at Different Outdoor Temperatures............................................................5 55 
3.2.5 LSH-SPM Development by Integrating Smart Dual Fuel Switching System Strategy

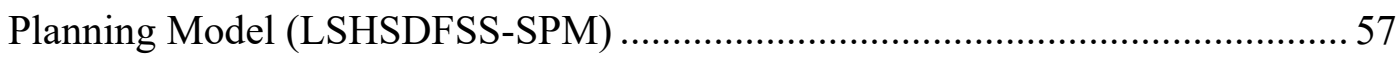

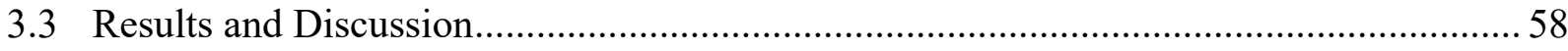

3.3.1 First Sample Day (January $\left.4^{\text {th }}, 2015\right)$ Simulation Result ........................................ 58

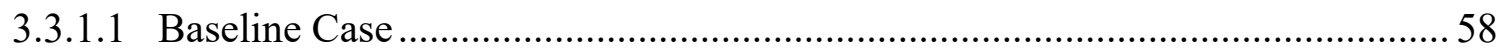

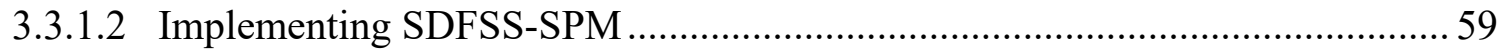

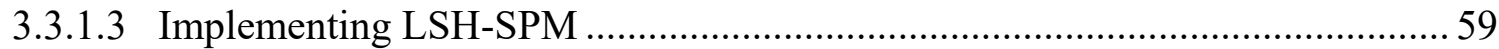

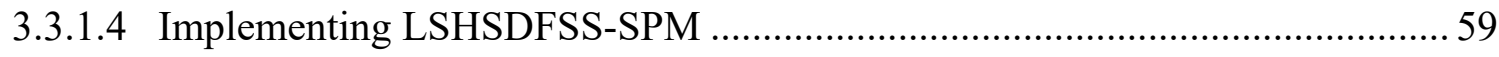

3.3.2 Comparing the Results of Different SPMs - First Sample Day .............................. 60

3.3.3 Comparing the Results of Different SPMs - Second Sample Day, January $5^{\text {th }} \ldots \ldots . . .60$

3.3.4 Comparing the Results of Different SPMs - Third Sample Day, January $6^{\text {th }} \ldots \ldots \ldots . . . .62$

3.3.5 Effectiveness of Various SPMs During Different Outdoor Temperatures ................ 63

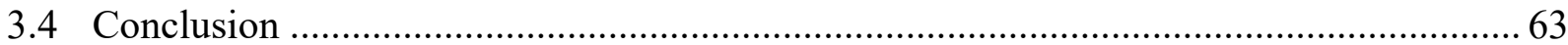

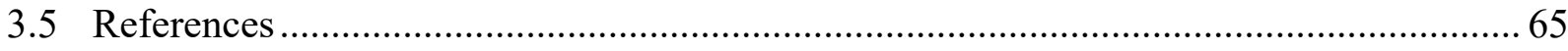

Chapter 4: Effects of Novel Energy Conservation Strategy Planning Models on HVAC System Energy Demand/Cost in the Heating and Cooling Seasons ..................................... 69

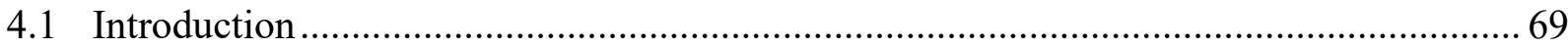

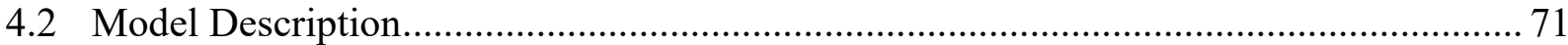

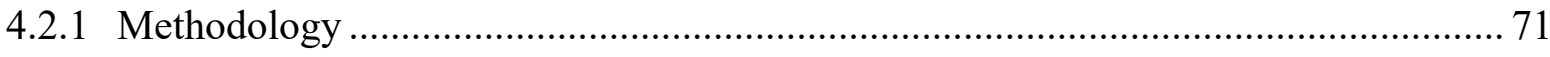

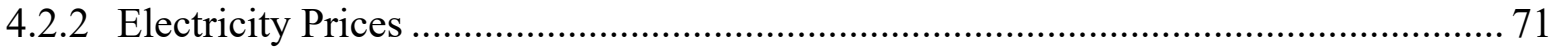

4.2.3 Using Load Shifting Strategy Planning Model during Summer (the cooling) Season 73

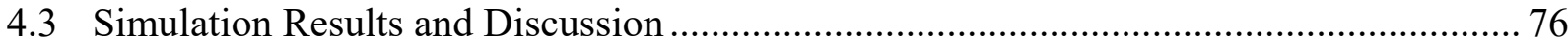

4.3.1 Analysis of the Impact of Intelligent Strategy Planning Models on HVAC System Demand and Energy Cost During Winter (the heating) Season ........................... 76

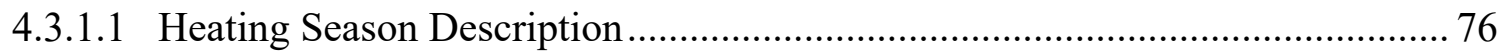

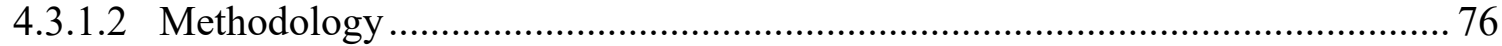

4.3.1.3 Impact of Different SPMs on the HVAC System Demand and Energy Cost on Three

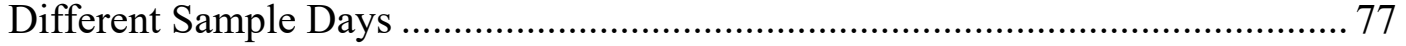

4.3.1.4 Energy Cost Saving Analysis by Utilizing Different SPMs in the Heating Season 81

4.3.1.5 Optimal Strategy Planning Model ............................................................. 83 
4.3.2 Impact of the Load Shifting Strategy Planning Model on HVAC System During Summer

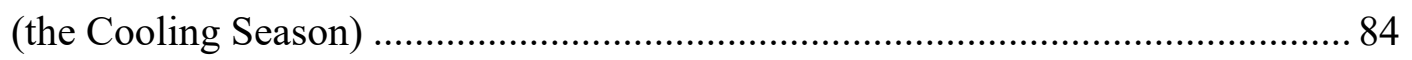

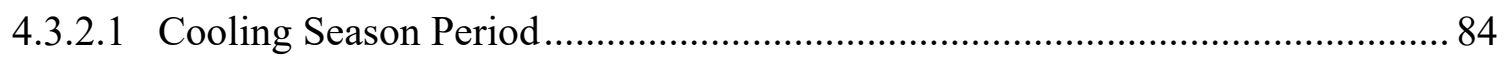

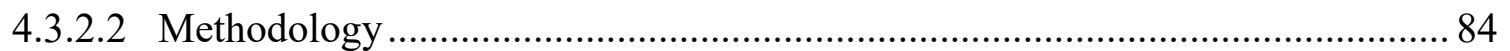

4.3.2.3 Impact of the LSH-SPM on HVAC System Demand and Energy Cost in the Cooling Season 84

4.3.2.4 Energy Cost Saving Analysis by Utilizing LSH-SPM in the Cooling Season .. 85

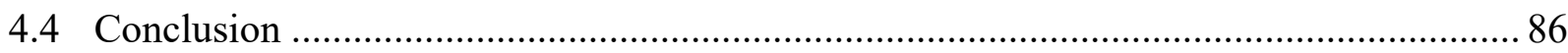

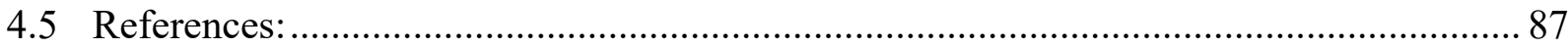

Chapter 5: Development of the Second MPC Controller to Determine Optimal HVAC Set Point

Profile Using On-site Renewable Energy Generation and Storage ............................ 89

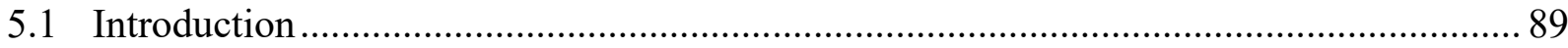

5.2 Model-based Predictive Controller Development........................................................... 93

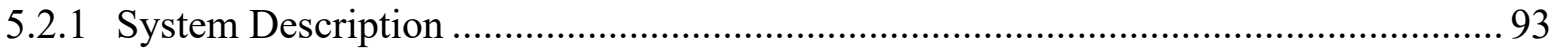

5.2.1.1 House Thermal Model Function ........................................................................ 93

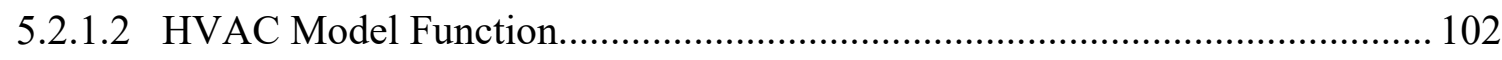

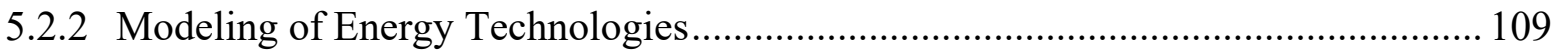

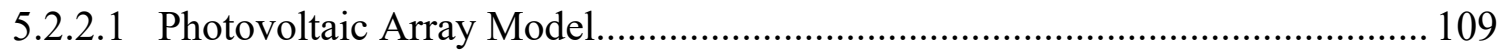

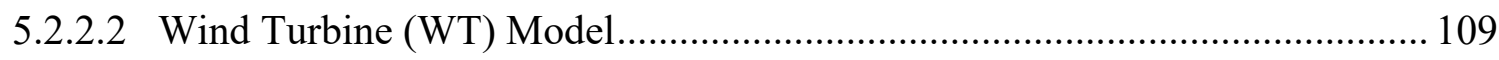

5.2.2.3 Battery Bank Model.................................................................................. 110

5.2.3 Model-based Predictive Controller Construction/Formulation.................................. 111

5.2.3.1 Overview on the Methodology of MPC Controller .......................................... 111

5.2.3.2 Executive Functions ....................................................................................... 116

5.2.3.2.1 House-HVAC Integrated Model Function............................................ 116

5.2.3.2.2 HVAC Smart Controller Function ....................................................... 116

5.2.3.2.3 Arranged Indoor Set Point Generator (AISPG) Function..................... 123

5.2.3.2.4 Comprehensive Set Point Matrix Generator (CSPMG) Function......... 126

5.2.3.2.5 Linear Optimization Function.............................................................. 131

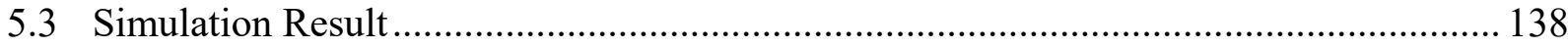

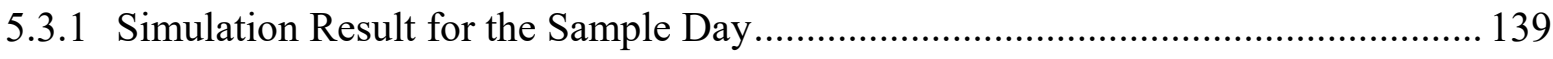

5.3.1.1 Conventional On-Off Controller................................................................... 140 


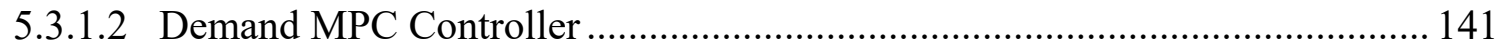

5.3.2 Simulation Result for the Heating Season ............................................................ 146

5.3.2.1 Base Case and Simple Rule-based Scenarios .................................................. 146

5.3.2.2 Employing Demand MPC Control Using PV, WT, and Battery Bank Systems 148

5.3.2.3 Employing Demand MPC Control without Using PV, WT, and Battery Bank

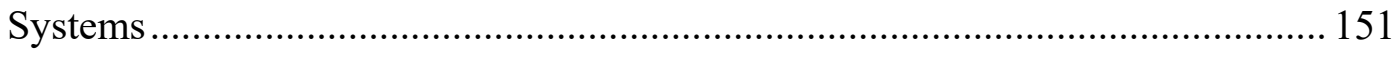

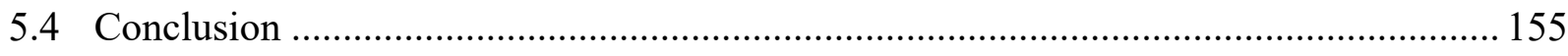

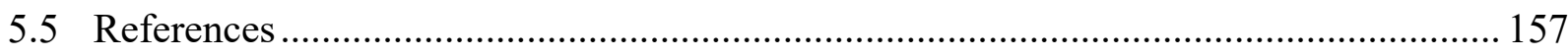

Chapter 6: Conclusions and Future Works ............................................................................. 161

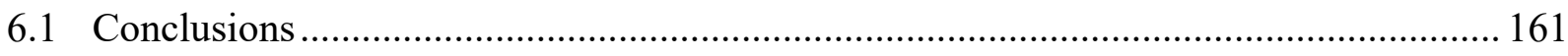

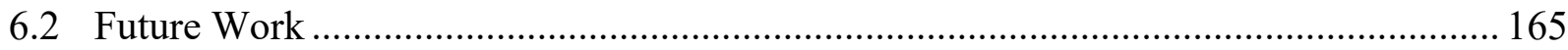

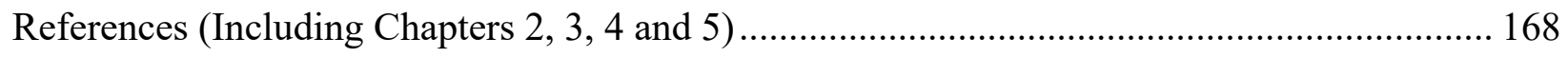




\section{List of Tables}

Table 3-1: Comparison between DFC before and after running SDFSS-SPM ........................ 49

Table 3-2: TRNSYS simulation results - Calculating TC factor ........................................... 52

Table 3-3: Impact of LSH-SPM on House A energy system in different outdoor temperatures.. 56

Table 3-4: TRNSYS simulation result after using weather forecast dataset-baseline................58

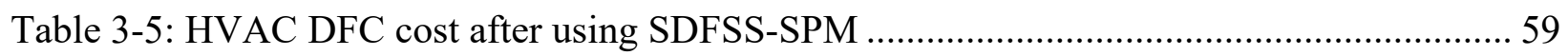

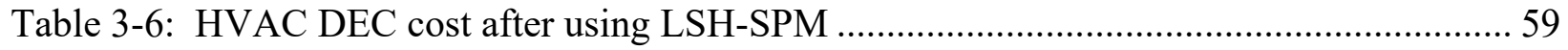

Table 3-7: HVAC DFC cost after using LSHSDFSS-SPM................................................ 59

Table 4-1: Compering the increment rates of TOU electricity prices ...................................... 72

Table 4-2: Incremental percentages of electricity prices between off-peak and peak hours ........ 72

Table 5-1: Azimuth angles for different walls of the house ............................................... 95

Table 5-2: Rates of correlation factors based on house properties/characteristics .................... 97

Table 5-3: House thermal model parameters/coefficients description ................................... 99

Table 5-4: Maximum capacity of renewable sources and total capacity of battery bank system111

Table 5-5: Optimization problem-weighting factor vector .............................................. 134

Table 5-6: Demand MPC controller set up information .................................................... 141

Table 5-7: Summary of the MPC controller energy cost saving with and without using PV, WT

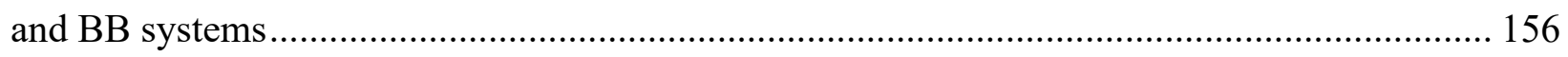

Table 6-1: Summary of simulation results for the first and the second MPC controllers ......... 164 


\section{List of Figures}

Figure 2-1: Different controlling techniques and functions used for constructing various system

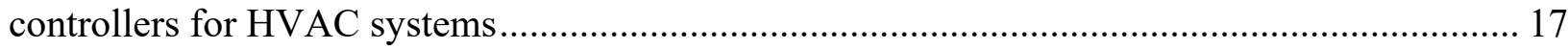

Figure 2-2: Different components of a HVAC system MPC controller ……………………….... 21

Figure 3-1: The overall view of various available weather datasets............................................. 38

Figure 3-2: The grid format of the GRIB2 data - Eastern Ontario............................................... 39

Figure 3-3: Framework of the house energy simulation system.................................................. 41

Figure 3-4: Framework of Matlab Controller Program .............................................................. 41

Figure 3-5: Operational command matrix generated with Matlab program after using weather

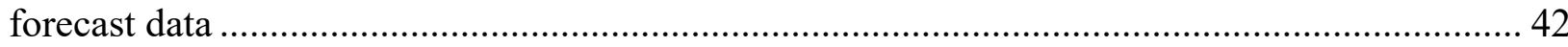

Figure 3-6: Outdoor temperature on Jan $4^{\text {th }}, 2015$ based on forecast weather dataset ................. 43

Figure 3-7: Operational command matrix graph - TRNSYS program.......................................... 43

Figure 3-8: Zone (1st floor) Temperature after running TRNSYS program with weather forecast data

Figure 3-9: Price of electricity in Ontario since November 1 ${ }^{\text {st }}$, 2014 for winter and summer ..... 44

Figure 3-10: House A ASHP COP validated with outdoor temperature ……………………..... 45

Figure 3-11: VIESMAN Co Technical Data Manual - Mini boiler efficiency curve ..................... 46

Figure 3-12: Switching points on different TOU prices ............................................................. 47

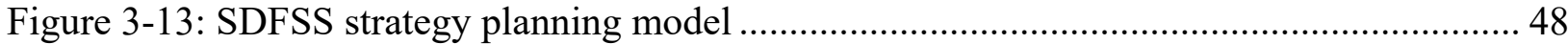

Figure 3-14: Developed operational command matrix after using SDFSS-SPM .......................... 48

Figure 3-15: Energy cost saved by using SDFSS-SPM at House A at different outdoor

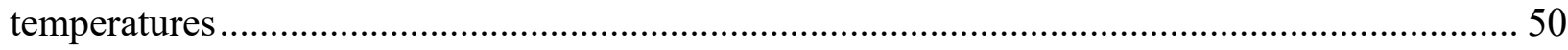

Figure 3-16: The operational mechanism of LSH-SPM model .................................................. 53

Figure 3-17: First operational command matrix generated based on BCS strategic plan ............. 54

Figure 3-18: Zone Temperature after running BCS operational command matrix ....................... 54

Figure 3-19: Final operational command matrix generated with Matlab program........................ 55

Figure 3-20: Energy cost saving using LSH-SPM at House A in different outdoor temperatures56

Figure 3-21: LSH-SPM additional/saving demand during different outdoor temperatures ......... 57

Figure 3-22: LSHSDFSS-SPM framework ……………….................................................... 57

Figure 3-23 : Developed operational command matrix after using LSHSDFSS-SPM ................. 58 
Figure 3-24: Comparing the potential energy cost savings of different SPMs on first sample day 60

Figure 3-25: Outdoor temperature on January $5^{\text {th }}, 2015$.

Figure 3-26: Comparing the potential energy cost savings of different SPMs on the second sample day 61

Figure 3-27: Outdoor temperature on January $6^{\text {th }}, 2015$. 62

Figure 3-28: Comparing the potential energy cost savings of different SPMs on the third sample day. 62

Figure 4-1: Price of electricity in Ontario as of November 1st, 2015 for winter and summer seasons 72

Figure 4-2: Zone temperature before implementing LSH-SPM. 74

Figure 4-3: Operational command matrix before implementing LSH-SPM .......................... 74

Figure 4-4: Zone temperature at the first iteration of LSH-SPM....................................... 75

Figure 4-5: Operational command matrix at the first iteration of LSH-SPM .......................... 75

Figure 4-6: Winter (the heating) season - outdoor temperature ............................................... 76

Figure 4-7: Outdoor temperature on December $1^{\text {st }}-$ TRNSYS metropolitan weather data ......... 77

Figure 4-8: HVAC system daily energy cost (DEC) and energy cost saving based on each

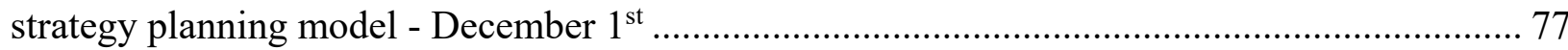

Figure 4-9: Outdoor temperature on January $1^{\text {st }}$ - TRNSYS metropolitan weather data ............ 78

Figure 4-10: HVAC system daily energy cost (DEC) and energy cost saving based on each

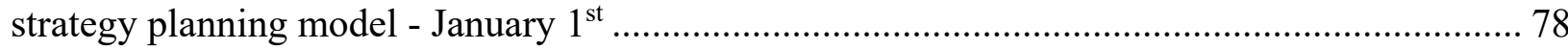

Figure 4-11: Outdoor temperature on February $1^{\text {st }}$ - TRNSYS metropolitan weather data ........ 79

Figure 4-12: HVAC system daily energy cost (DEC) and energy cost saving based on each strategy planning model - February $1^{\text {st }}$ 79

Figure 4-13: The outdoor temperature on the coldest day of year - TRNSYS metropolitan weather data. 80

Figure 4-14: HVAC system daily energy cost (DEC) and energy cost saving based on each strategy planning model on the coldest day of the year..... 80 Figure 4-15: DEC of HVAC system based on different strategy planning models in the heating season. 81

Figure 4-16: Energy cost saving with different strategy planning models in the heating season. 82 
Figure 4-17: Minimum daily operating cost of HVAC system - heating season days ............... 83

Figure 4-18: Summer (the cooling) season - outdoor temperature ......................................... 84

Figure 4-19: DEC of HVAC system before and after taking advantage of LSH-SPM in the

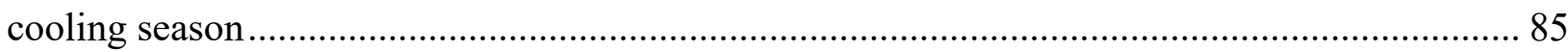

Figure 4-20: Energy cost saving implementing LSH strategy planning model in the cooling

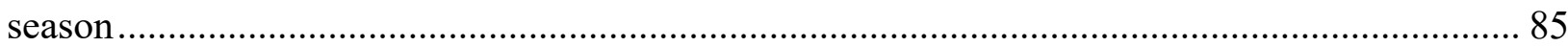

Figure 5-1: Solar radiation (SR) on different orientations of House A during heating season .. 100

Figure 5-2: Outdoor air temperature and wind speed during the heating season .................... 101

Figure 5-3: House thermal demand (Qz) during the heating season .................................... 102

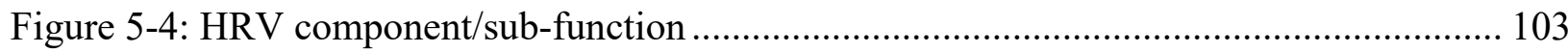

Figure 5-5: Trendline between cold outdoor air temperatures and pre-heated fresh air temperature

$-\mathrm{HRV}$ 104

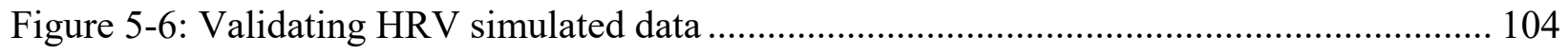

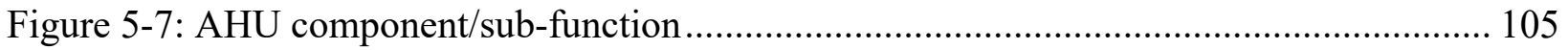

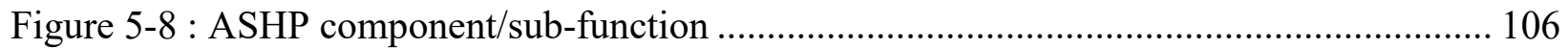

Figure 5-9: Mini boiler efficiency curves provided by the manufacturer .............................. 107

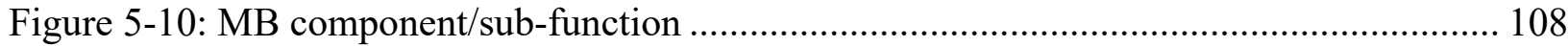

Figure 5-11: House-HVAC integrated model schematic diagram...................................... 108

Figure 5-12: Framework of Demand MPC controller ....................................................... 115

Figure 5-13: Zone temperature oscillation during 3 hours control time horizon...................... 117

Figure 5-14: Zone temperatures by choosing 1-minute and 30-minute as the time steps ......... 118

Figure 5-15: Switching times of HVAC system after selecting 1-minute and 30-minute time steps 118

Figure 5-16: Framework/structure of the exhaustive optimization core ................................ 120

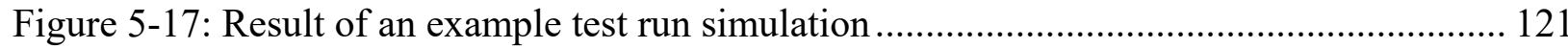

Figure 5-18: Framework of HVAC smart controller function.......................................... 122

Figure 5-19: A sample of HVAC system optimum demand profile with 24-hour control time horizon

Figure 5-20: Arranged indoor set point vs. HVAC system demand .................................... 125

Figure 5-21: Three different TOU price switching points in winter season in Ontario............. 127

Figure 5-22: Degrees of freedom in first zone hours ....................................................... 128 
Figure 5-23: Generated set point patterns matrix containing nine states..... 128

Figure 5-24: Rearranging the indoor set points in the first state of comprehensive matrix........ 130

Figure 5-25: HVAC system energy demand using arranged and first state of comprehensive matrix set point patterns. 130

Figure 5-26: Zone temperature in entire day under the effects of last state indoor set point pattern

Figure 5-27: Input and output variables of the linear optimization function. 132

Figure 5-28: Energy flow management diagram of the case study house 135

Figure 5-29: Collecting all the optimum results to prepare the optimum vector..... 138

Figure 5-30: Solar irradiation on different orientations of the case study house. 139

Figure 5-31: Outdoor temperature and wind speed during the sample day.... 139

Figure 5-32: Case study house thermal demand during the sample day. 140

Figure 5-33: ASHP energy demand during the sample day ..... 140

Figure 5-34: HVAC system minimum energy cost and optimum operating time step 141

Figure 5-35: Average of HVAC system energy cost in each control TH 142

Figure 5-36: Optimum indoor set point pattern along with corresponding HVAC system energy

demand and TOU energy price scheme 143

Figure 5-37: Renewable energy generated by PV and WT during the sample day..... 144

Figure 5-38: Optimum powers drawn from different energy sources during the sample day .... 144

Figure 5-39: Contribution of each energy source in supplying the HVAC system energy demand

Figure 5-40: Savings on the HVAC system energy cost after implementing the Demand MPC controller 146

Figure 5-41: Indoor set point profiles base case different four scenarios 147

Figure 5-42: Energy cost of HVAC system by using different base case scenarios in heating season. 148

Figure 5-43: Comparing MPC result with different base case and SRB scenarios for the heating season 148

Figure 5-44: Comparing HVAC system energy cost based on SRB scenario and MPC controller during the heating season 149 
Figure 5-45: MPC additional saving on the HVAC system energy cost (comparing SRB scenario) during different days of heating season

Figure 5-46: HVAC system energy demand based on SRB scenario and after employing MPC controller during the heating season 150

Figure 5-47: Saving on the HVAC system energy demand (comparing SRB scenario) after employing MPC controller during the heating season

Figure 5-48: Optimum HVAC system operating time steps during the heating season.

Figure 5-49: Comparing MPC result with SRB scenario in the heating season 152 Figure 5-50: Comparing HVAC system energy cost based on MPC controller and SRB scenario in the heating season 152

Figure 5-51: Additional daily energy cost saving of MPC controller in comparison with SRB scenario during the different days of the heating season

Figure 5-52: Outdoor temperature and wind speed in day141

Figure 5-53: Solar radiation on the house different orientations in day 141 154

Figure 5-54: HVAC system energy demand based on SRB scenario 154 Figure 5-55: Optimum indoor set point profile and corresponding HVAC energy demand after employing the MPC controller 155 


\section{List of Appendices}

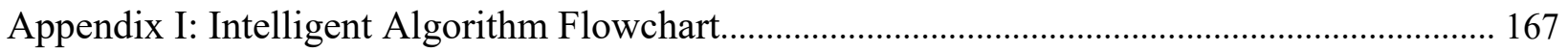




\section{Abbreviations}

\begin{tabular}{|c|c|}
\hline $\mathrm{AE}$ & Absolute Error \\
\hline AISPG & Arranged Indoor Set Point Generator \\
\hline $\mathrm{AHU}$ & Air Handling Unit \\
\hline ANN & Artificial Neural Network \\
\hline ARIMA & Autoregressive Integrated Moving Average \\
\hline ARMA & Autoregressive Moving Average \\
\hline ARMAX & Autoregressive Moving Average Exogenous \\
\hline AR & Autoregressive \\
\hline ARI & Autoregressive Integrated \\
\hline ARX & Autoregressive Exogenous \\
\hline ASH & Archetype Sustainable House \\
\hline ASHP & Air Source Heat Pump \\
\hline ASHRAE & $\begin{array}{l}\text { American Society of Heating, Refrigerating and Air-Conditioning } \\
\text { Engineers }\end{array}$ \\
\hline $\mathrm{BB}$ & Battery Bank \\
\hline BCS & Best Case Scenario \\
\hline $\mathrm{CC}$ & Correlation Coefficient \\
\hline $\mathrm{CMC}$ & Canadian Meteorological Centre \\
\hline COP & Coefficient of Performance \\
\hline CSPMG & Comprehensive Set Point Matrix Generator \\
\hline DAQ & Data Acquisition System \\
\hline $\mathrm{DEC}$ & Daily Electricity Cost \\
\hline DFC & Daily Fuel Cost \\
\hline DHW & Domestic Hot Water \\
\hline DMPC & Deterministic Model-based Predictive Controller \\
\hline DSO & Distribution System Operators \\
\hline ECSPM & Energy Conservation Strategy Planning Model \\
\hline FL & Fuzzy Logic \\
\hline FWD & Forecast Weather Dataset \\
\hline GA & Genetic Algorithm \\
\hline HRDPS & High Resolution Deterministic Prediction System \\
\hline HRV & Heat Recovery Ventilator \\
\hline HVAC & Heating, Ventilating and Air Conditioning \\
\hline IAG & Indoor Air Quality \\
\hline
\end{tabular}




\begin{tabular}{|c|c|}
\hline LEED & Leadership in Energy and Environmental Design \\
\hline LO & Linear Optimization \\
\hline LSH & Load Shifting \\
\hline LSHSDFSS & Load Shifting and Smart Dual Fuel Switching System \\
\hline MB & Mini Boiler \\
\hline $\mathrm{ME}$ & Maximum Exploitation \\
\hline MIMO & Multi Input Multi Output \\
\hline MPC & Model-based Predictive Controller \\
\hline MSC & Meteorological Service of Canada \\
\hline NCOT & Nominal Cell Operating Temperature \\
\hline NRCan & Natural Resource Canada \\
\hline NZEB & Net Zero Energy Building \\
\hline OD & Outdoor Disturbance \\
\hline OEB & Ontario Energy Board \\
\hline OT & Outdoor Temperatures \\
\hline PI & Proportional Integral \\
\hline PID & Proportional Integral Derivative \\
\hline PHEV & Plug-in Hybrid Electric Vehicle \\
\hline PMOC & Pulse Modulation Adaptive Controller \\
\hline PMV & Predicted Mean Vote \\
\hline PV & Photovoltaic Panel \\
\hline $\mathrm{RBC}$ & Rule-based Control \\
\hline $\mathrm{RH}$ & Residential House \\
\hline RPM & Revolutions Per Minute \\
\hline SDFSS & Smart Dual Fuel Switching System \\
\hline SMPC & Stochastic Model-based Predictive Controller \\
\hline SP & Set Point \\
\hline SPM & Strategy Planning Model \\
\hline $\mathrm{SOC}$ & State of Charge \\
\hline SRB & Simple Rule-based \\
\hline STD & Standard Deviation \\
\hline STC & Standard Test Condition \\
\hline $\mathrm{TC}$ & Time Constant \\
\hline $\mathrm{TH}$ & Time Horizon \\
\hline TM & Thermal Mass \\
\hline
\end{tabular}




$\begin{array}{ll}\text { TOU } & \text { Time of Use } \\ \text { TRCA } & \text { Toronto and Region Conservation Authority } \\ \text { TS } & \text { Time Step } \\ \text { VAV } & \text { Variable Air Volume } \\ \text { WF } & \text { Weighting Factor } \\ \text { WFD } & \text { Weather Forecast Data } \\ \text { WT } & \text { Wind Turbine } \\ \text { ZT } & \text { Zone Temperature }\end{array}$




\section{Nomenclature}

Alphabets

\begin{tabular}{|c|c|}
\hline$A$ & Un-shaded area of windows' glass $\left(\mathrm{m}^{2}\right)$ \\
\hline$A_{e q}$ & $\begin{array}{l}\text { Coefficient matrix of decision making variables in equality } \\
\text { equations }\end{array}$ \\
\hline$A C H_{\text {avg }}$ & Annual average air exchange rate \\
\hline $\mathrm{ACH}_{50}$ & Hourly air exchange rate at 50 Pascal \\
\hline$A F_{A H U}$ & AHU supplied air flow rate $(\mathrm{kg} / \mathrm{s})$ \\
\hline$A F_{H R V}$ & HRV supply and return air flow rate $(\mathrm{kg} / \mathrm{s})$ \\
\hline$A F_{r z}$ & Return (zone) air flow rate $(\mathrm{kg} / \mathrm{s})$ \\
\hline$A T$ & Dry-bulb air temperature $\left({ }^{\circ} \mathrm{C}\right)$ \\
\hline$A T_{f o}$ & Fresh outdoor air temperature $\left({ }^{\circ} \mathrm{C}\right)$ \\
\hline$A T_{m a}$ & Mixed box air temperature $\left({ }^{\circ} \mathrm{C}\right)$ \\
\hline$A T_{p f}$ & Pre-heated fresh air temperature $\left({ }^{\circ} \mathrm{C}\right)$ \\
\hline$A T_{r z}$ & Return zone air temperature $\left({ }^{\circ} \mathrm{C}\right)$ \\
\hline$A T_{\text {su }}$ & AHU supplied air temperature $\left({ }^{\circ} \mathrm{C}\right)$ \\
\hline$A_{w l_{i}}$ & Area of the $i^{\text {th }}$ wall $\left(\mathrm{m}^{2}\right)$ \\
\hline$A_{w d_{i}}$ & Area of the $i^{t h}$ windows $\left(\mathrm{m}^{2}\right)$ \\
\hline$b$ & Vector of inequality equations \\
\hline$b_{e q}$ & Vector of equality equations \\
\hline$C_{\text {air }}$ & Specific heat of air at constant pressure (kJ/kg.K) \\
\hline$C_{n}$ & Nominal capacity of battery bank (Ah) \\
\hline$C L F$ & Cooling load factor \\
\hline COV & Covariance function \\
\hline$D_{i}$ & Total house electricity demand at $i^{\text {th }}$ time step $(\mathrm{kW})$ \\
\hline Density & Density of thermal mass material $\left(\mathrm{kg} / \mathrm{m}^{3}\right)$ \\
\hline$F F$ & PV module fill factor \\
\hline$H_{1}$ & Building height correction factor \\
\hline$h_{o_{i}}$ & $\begin{array}{l}\text { Overall external surface coefficient of } i^{t^{h}} \text { wall/windows } \\
\left(\mathrm{W} / \mathrm{m}^{2} \cdot{ }^{\circ} \mathrm{C}\right)\end{array}$ \\
\hline$I$ & $\begin{array}{l}\text { Intensity of incident solar radiation at external surface of } i^{\text {th }} \\
\text { wall/windows }\left(\mathrm{W} / \mathrm{m}^{2}\right)\end{array}$ \\
\hline$I_{s c}$ & PV cell short circuit current $(A)$ \\
\hline$I_{S C, s t c}$ & PV cell short circuit current at standard test condition (A) \\
\hline$G_{P}$ & Solar irradiance $\left(\mathrm{W} / \mathrm{m}^{2}\right)$ \\
\hline
\end{tabular}




\begin{tabular}{|c|c|}
\hline$K_{I}$ & PV cell short circuit current temperature coefficient \\
\hline$K_{V}$ & PV cell open circuit voltage temperature coefficient \\
\hline$I b$ & Lower bounds of decision making variables \\
\hline$L W R_{i}$ & $\begin{array}{l}\text { Temperature drop on the surface of } i^{t h} \text { wall/windows due to } \\
\text { long-wave radiation }\left({ }^{\circ} \mathrm{C}\right)\end{array}$ \\
\hline$L_{3}$ & Leakiness correction factor \\
\hline $\operatorname{MaxSP}$ & User-defined maximum set point $\left({ }^{\circ} \mathrm{C}\right)$ \\
\hline MinSP & User-defined minimum set point $\left({ }^{\circ} \mathrm{C}\right)$ \\
\hline$N_{0}$ & Leakage-infiltration factor \\
\hline$N_{p}$ & Number of parallel PV modules \\
\hline$N_{s}$ & Number of series PV modules \\
\hline$P_{B b}$ & Battery bank output energy (kWh) \\
\hline$P_{B b D_{i}}$ & Energy drawn from battery bank at $i^{\text {th }}$ time step $(\mathrm{kWh})$ \\
\hline$P_{B b-I n i t i a l}$ & Initial SOC of battery bank system (kWh) \\
\hline$P_{C h B b_{i}}$ & $\begin{array}{l}\text { Energy used for charging the battery bank at } i^{\text {th }} \text { time step } \\
(\mathrm{kWh})\end{array}$ \\
\hline$P_{L p_{i}}$ & Demand of non-HVAC house loads at $i^{\text {th }}$ time step (kWh) \\
\hline$P_{L g D_{i}}$ & Energy drawn from local grid at $i^{\text {th }}$ time step (kWh) \\
\hline$P_{L G-M a x}$ & Maximum capacity of local grid ( $\mathrm{kWh})$ \\
\hline$P_{P v}$ & PV output energy (kWh) \\
\hline$P_{P V-M a x}$ & Maximum capacity of PV $(\mathrm{kW})$ \\
\hline$P_{P v D_{i}}$ & Energy drawn from PV at $i^{\text {th }}$ time step $(\mathrm{kWh})$ \\
\hline$P_{S C-\operatorname{Max}}$ & Maximum capacity of solar community $(\mathrm{kW})$ \\
\hline$P_{S c D_{i}}$ & Energy drawn from solar community at $i^{\text {th }}$ time step $(\mathrm{kWh})$ \\
\hline$P_{W t}$ & WT output energy $(\mathrm{kWh})$ \\
\hline$P_{W T-M a x}$ & Maximum capacity of WT (kW) \\
\hline$P_{W t D_{i}}$ & Energy drawn from WT at $i^{\text {th }}$ time step $(\mathrm{kWh})$ \\
\hline$Q_{a p}$ & Appliance heat gain $(\mathrm{kWh})$ \\
\hline Qele & ASHP electricity demand (kWh) \\
\hline$Q_{H R V}$ & HRV recovered thermal energy $(\mathrm{kWh})$ \\
\hline$Q_{\text {inf }}$ & Infiltration heat loss energy $(\mathrm{kWh})$ \\
\hline$Q_{n g}$ & Mini boiler natural gas demand ( $\mathrm{kWh})$ \\
\hline$Q_{s o}$ & Solar heat gain energy $(\mathrm{kWh})$ \\
\hline$Q_{s u}$ & HVAC supplied thermal energy (kWh) \\
\hline$Q_{w d}$ & Windows conductive thermal energy $(\mathrm{kWh})$ \\
\hline$Q_{w l}$ & Wall conductive thermal energy $(\mathrm{kWh})$ \\
\hline
\end{tabular}




$\begin{array}{ll}Q_{z} & \text { Zone/house thermal energy demand }(\mathrm{kWh}) \\ S H & \text { Specific heat }(\mathrm{kJ} / \mathrm{kg} . \mathrm{K}) \\ S H G C & \text { Solar heat gain coefficient } \\ S H G F_{m a x} & \text { Maximum solar heat gain factor } \\ S_{2} & \text { Site shielding factor } \\ T C & \text { Cell temperature }\left({ }^{\circ} \mathrm{C}\right) \\ T_{o} & \text { Outdoor air temperature }\left({ }^{\circ} \mathrm{C}\right) \\ T_{s a_{i}} & \text { Sol-air temperature at the surface of } i^{\text {th }} \text { wall } / \text { window }\left({ }^{\circ} \mathrm{C}\right) \\ T_{w l_{i}} & \text { Temperature of the external surface of } i^{\text {th }} \text { wall }\left({ }^{\circ} \mathrm{C}\right) \\ T_{w d_{i}} & \text { Temperature of the external surface of } i^{\text {th }} \text { window }\left({ }^{\circ} \mathrm{C}\right) \\ T_{z} & \text { Temperature of the zone }\left({ }^{\circ} \mathrm{C}\right) \\ u b & \text { Upper bounds of the decision making variables } \\ U_{w l_{i}} & \text { Overall heat convection coefficient of the } i^{t h} \text { wall }\left(\mathrm{W} / \mathrm{m}^{2} .{ }^{\circ} \mathrm{C}\right) \\ U_{w d_{i}} & \text { Overall heat convection coefficient of the } i^{t h} \text { window } \\ V_{c i} & \left.\text { (W/m }{ }^{\circ}{ }^{\circ} \mathrm{C}\right) \\ V_{c o} & \text { WT cut-in wind speed }(\mathrm{m} / \mathrm{s}) \\ V_{r} & \text { WT cut-out wind speed }(\mathrm{m} / \mathrm{s}) \\ V_{o c} & \text { Rated wind speed }(\mathrm{m} / \mathrm{s}) \\ V_{o c, s t c} & \text { PV cell open circuit voltage }(\mathrm{V}) \\ V_{W} & \text { PV cell open circuit voltage at standard test condition }(\mathrm{V}) \\ V_{Z} & \text { Wind speed at the hub height }(\mathrm{m} / \mathrm{s}) \\ W_{s} & \text { Volume of the zone }\left(\mathrm{m}^{3}\right) \\ & \text { Wind speed }(\mathrm{m} / \mathrm{s})\end{array}$

Greek Letters

$\begin{array}{ll}\alpha & \text { Power law exponent factor } \\ \alpha_{i} & \text { Absorptivity of external surface } i^{t h} \\ \rho_{a i r} & \text { Air density }\left(\mathrm{kg} / \mathrm{m}^{3}\right) \\ \rho_{m a} & \text { Air density at mixing box }\left(\mathrm{kg} / \mathrm{m}^{3}\right) \\ \rho_{s u} & \text { Air density at the outlet of AHU (supplied air) }\left(\mathrm{kg} / \mathrm{m}^{3}\right) \\ \sigma_{S_{i}} & \text { Standard deviation of } i^{\text {th }} \text { series of data } \\ \eta_{B} & \text { Battery round-trip efficiency } \\ \eta_{i n v} & \text { Inverter efficiency } \\ \eta_{s} & \text { HRV sensible thermal efficiency } \\ \eta_{m b} & \text { Mini boiler efficiency } \\ \Delta T C_{\text {wind }} & \text { Change in cell temperature under the wind effect }\left({ }^{\circ} \mathrm{C}\right)\end{array}$




\section{Chapter 1: Introduction}

\subsection{Current Problem Statements}

One of the most crucial problems for the power networks is peak load. Peak load threatens the quiddity of power networks with short-term systematic disorders, including grid instability, severe voltage fluctuation, grid failure, and system blackout. In addition to systematic error, peak load has a strong influence on the cost of the produced electricity. This can be observed in Time of Use (TOU) and real-time pricing schemes. Although peak power is used only for very short periods of time, it has to be delivered continuously by the utility, despite the high cost of delivery (since electricity suppliers and transmission lines are usually sized to correspond to peak load demand) and its destructive impact on the environment.

In addition to peak hours, off-peak hours also have destructive impacts on the power grid. During off-peak hours, power plants are forced to turn off some of their generators. Therefore, leaving efficient power plants off or having them run at less than their full capacity (maximum profit) during off-peak hours decreases their total power efficiency and wastes capital investment as well. Due to the rapid growth of infrastructure development, the destructive impacts of peak load on power networks will be even greater in the future. Hence, a considerable financial investment is necessary to develop infrastructure, i.e., new power plants and distribution networks.

In addition to peak and off-peak problems, building energy simulators such as TRNSYS, EnergyPlus, and esp-r suffer from poor control mechanism and are unable to employ load shifting strategy planning models. In other words, these software platforms do not include sub-models of advanced controllers/strategies for employing and addressing energy conservation strategy planning models (i.e., load shifting and fuel switching systems) to manage the demand (support the local grid) by reducing peak load and increasing off-peak load.

A model-based predictive controller (MPC), as one of the strongest system control mechanisms, can employ/address energy conservation strategy planning sub-models in the system model. However, since MPC models examine multiple scenarios within the defined process space to determine the optimum state of the system, they suffer from unacceptably long computational (optimization) time. 


\subsection{Motivation}

The objective of this research is to develop a grid-friendly residential building that supports the local grid during peak and off-peak hours while minimizing total energy costs. To this end, the controller should be designed with the aim of maximizing the usage of renewable energy while managing internal load in order to minimize the demand during peak hours and maximize it during off-peak hours. The thesis objectives could be achieved by developing feasible model-based predictive controllers (MPCs) for residential house HVAC systems. The MPC model should be used to consider the future state of the model into an optimization scheme. In comparison with the conventional (on/off, PI and PID) system controller, the MPC is much more powerful and adaptable. The MPC provides an anticipatory control mechanism by predicting the future state of the system based on the estimate of component behaviors and produces a constrained control matrix which drives the system towards the optimum state. The MPC finds the optimal solution for different optimization problems by using a cost function (i.e., energy consumption or energy cost). The developed MPC controller, called Demand MPC controller, controls the supplied renewable energies and the energy stored in the battery bank system; it manages the system's total energy flow and considers the effect of the TOU pricing scheme and Weather Forecast Data (WFD) on the optimization problem. Furthermore, this controller minimizes the demand and/or energy cost of the HVAC system, taking into consideration the TOU pricing and the outdoor disturbances (OD) effects on the optimization scheme. The controller examines many different possible scenarios within the defined space and finds the best control strategy (indoor set point profile) for each control time horizon without compromising thermal comfort. Using the WFD, the MPC controller predicts the potential behavior of renewable energy (PV, WT) suppliers and load consumers in advance to find the optimal operational mechanism for running the HVAC system. After designing the MPC model, different operational SPMs (such as Load Shifting (LSH) and Smart Dual Fuel Switching System (SDFSS)) could be applied in the system to achieve the thesis objectives. After solving the optimization problems, the optimal operational SPM which conserves all the objectives would be determined.

In summary, the main motivations for developing the optimal operational SPM using MPC controllers in the context of a grid-friendly house are as follows: 
- Taking advantage of MPC model on supply, storage, and demand sides

- Using load shifting SPM in order to support local grids during peak hours by incorporating the TOU pricing variable in the optimization problem and considering passive thermal storage capacitance of the house.

- Supporting the local grid during off-peak hours system in order to draw the electricity from the grid during off-peak hours.

- Minimizing the electricity consumption and total energy cost considering electricity TOU pricing scheme.

- Maximizing the usage of renewable energy in order to minimize the usage of battery bank systems, which are relatively expensive systems with high maintenance requirements.

- Prediction of the future states of the system consider the system's process model and producing control matrix which drives the system towards the optimum state.

- Solving optimization programs to find the optimal operational SPM and indoor set point profile.

- Developing the optimal model by considering the effect of outdoor disturbances

- Designing a grid-friendly residential house

\subsection{Challenges}

The main objective of this research is to design the MPC controller for residential HVAC systems to reduce the operating cost by employing energy conservation strategy planning models (i.e. load shifting and fuel switching system) without compromising the occupants' thermal comfort. Some of the main challenges in designing the MPC control for HVAC systems are listed below:

- Enhancing the poor control mechanism of building energy simulators by designing an advanced MPC co-simulator.

- Finding access to accurate WFD as feed-in variables of renewable energy suppliers and load consumer models

- Developing novel energy conservation SPMs and operational optimization methods into the simulation framework 
- Capturing appropriate system models suitable for MPC design using physics based, black and gray box modeling approaches

- Simulation framework design to cover the overall house energy systems including house model, solar energy suppliers, load consumers, energy balancing system, storage system and so on

- Considering the impacts of multiple outdoor disturbance variables on house thermal model and optimization process

- Solving the total optimization scheme considering different kinds of optimization problems with different constraints

- Solving optimization problems considering different HVAC operating time steps and MPC control time horizons

- Reducing the MPC computational (optimization) time by extracting the useful indoor set point patterns/profiles.

All of the challenges mentioned above, were overcome during the course of this research for the development of MPC controllers.

\subsection{Style of the Dissertation}

This dissertation follows the manuscript-style dissertation guidelines of the Mechanical and Industrial Engineering Department. It satisfies the following criteria of the manuscript-style dissertation:

1. Dissertation contains three journal papers each making an original and significantly different contribution.

2. The student is the principal author of the papers.

3. All the work reported in the journal papers and this dissertation is primarily contributed by the first author. The student is prepared to stand for, and defend all the work included in this dissertation. 


\subsection{Publications}

Following is a list of the published journal and conference papers, which are the part of this dissertation.

Full LeNGTH, FUlLy REFEREED JOURNAL PAPERS ( PUBLISHED/IN PRESS)

1. Nima Alibabaei, Alan S. Fung, Kaamran Raahamifar, "Optimal Residential HVAC Set Point Profile Using Outdoor Disturbances and HVAC Operating Time Step with On-site Energy Generation and Storage”, Applied Energy, 2017 (In Press).

2. Nima Alibabaei, Alan S. Fung, Kaamran Raahamifar, Arash Moghimi, "Effects of Intelligent Strategy Planning Models on Residential HVAC System Energy Demand and Cost during the Heating and Cooling Seasons”, Applied Energy, Vol 185 (1), 2017, pp: 2943

3. Nima Alibabaei, Alan S. Fung, Kaamran Raahamifar, "Development of Matlab-TRNSYS Co-simulator for Applying Predictive Strategy Planning Models on Residential House HVAC System”, Energy and Buildings, Vol 128, 2016, pp: 81-98.

\subsection{Structure of the Dissertation}

This thesis is divided into six chapters:

In Chapter 2, a review of the existing control methodologies that applied in HVAC and residential house energy systems is conducted. This chapter also reviews different kinds of model-based predictive controllers (MPCs) including their infrastructures and internal components. HVAC modeling methods highlighting several modeling techniques such as the black-box, white-box, and gray-box models have also been reviewed in this chapter.

Chapter 3 describes the development of first MPC controller using Matlab-TRNSYS Co-simulator. In this MPC model, comprehensive process/plant models have been used for house thermal energy and HVAC system using TRNSYS program. This chapter also discusses the development of novel predictive energy conservation strategy planning models (SPMs) including Load Shifting (LSH), Smart Dual Fuel Switching System (SDFSS), and LSHSDFSS, as the integration of fuel switching and load shifting strategy planning models. This chapter investigates the effectiveness of 
developed predictive SPMs on three sample days of the winter season. Chapter 3 covers the contents of the first journal paper that was published in the Journal of Energy and Buildings.

Development of the Matlab-TRNSYS Co-simulator for including/covering the cooling (summer) season is discussed in Chapter 4. This chapter also investigates the effects of novel energy conservation strategy planning models on HVAC system energy demand/cost in heating and cooling seasons using Ontario 2015 time of use pricing scheme. Chapter 4 covers the contents of the second journal paper that was published in the Journal of Applied Energy. Arash Moghimi, as one of the journal paper co-authors, has assisted in the development of a mechanism for connecting Matlab to TRNSYS program.

Chapter 5 discusses the development of second MPC controller as a novel model-based predictive control (MPC) scheme, which is developed to determine the optimal HVAC system set point profile. This novel MPC controller is developed with the objective of reducing residential HVAC systems energy demand and energy cost while maximizing the use of renewable energies. In this MPC controller, simplified process/plant models were developed and used for house thermal energy and HVAC system. Chapter 5 also compares the result of developed (second) MPC controller on energy demand and cost savings with base case and Simple Rule-based (SRB) logics/controllers. Chapter 5 covers the contents of third journal paper that has been submitted to the Journal of Applied Energy.

Conclusions and future works are highlighted in Chapter 6. 


\section{Chapter 2: Review of Control Methods and Energy Conservation Strategy Planning Models in Residential Houses and HVAC Systems}

This chapter presents the literature review of the methods used to control and implement energy conservation strategy planning models (ECSPMs) in the residential houses/buildings and their HVAC systems. The development of control methods and ECSPM control strategies are required to: improve the HVAC system energy efficiency, reduce the HVAC system energy demand and cost, support the local grid/utility, and protect the environment. Controlling the performance and operation of HVAC systems is very momentous and vital since they must provide secure and uninterruptible thermal comfort for the occupants. Controlling the HVAC systems is also complex because of the complexity of house thermal energy system, HVAC system structures, and the effects of various parameters like indoor and outdoor disturbances on the house thermal energy system. To build a process/plant model (i.e., for predicting the behavior of HVAC system), one needs to model the house thermal energy system as well as HVAC components. This can be performed either by using house thermal energy simulators like TRNSYS, EnergyPlus, and esp-r or by taking advantage of simplified house thermal energy and HVAC system models using different classes of physics-based, data driven, and gray-box models. In order to control the process/plant model (i.e., controlling the house thermal comfort), a control platform/infrastructure should be developed to manage HVAC system performance while facilitating the implementation of different ECSPM control strategies in the HVAC system. In this chapter, major control platforms/techniques and energy conservation strategy planning models applied in residential HVAC systems and reported in the recent literature have been reviewed.

\subsection{Introduction}

Controlling a building's HVAC system is vital for providing thermal comfort for occupants (by regulating the quality of indoor environment). It is also necessary for regulation of energy consumption and optimization of energy cost. Generally, different types of controlling approaches are used for HVAC systems including supervisory level control, local level control, 
classical/conventional control, hard and soft controls, hybrid control, other control, and modelbased predictive control.

In supervisory level control, the control set points are generated in the supervisory level controllers in order to manage the lower level controllers. For example, in the house level, these set points can be determined for HVAC and non-HVAC loads. In local level control, the local level controllers mainly control the variables within the set point ranges determined by the supervisory control levels. However, because of the complexity of HVAC systems, local level controllers must look after indoor air quality (IAQ) variables to ensure ASHRAE indoor thermal comfort standard. In classical/conventional control, including on/off, P, PI, and PID controllers, the goal is to control the process within the boundaries. To this end, these controllers run and conduct the processes using the error dynamic function. In hard control, including robust control, nonlinear control, and gain scheduling techniques, the processes are regulated based on the theory of control systems. In other words, in hard controllers, the optimal control algorithm will find a solution for an optimization problem based on an appropriate cost function. In soft control level, including fuzzy logic and neural network based controllers; a comprehensive operational databank of the plant is used for training the network. The hybrid controllers are developed by combining the techniques of hard and soft controllers. In other control level, new control techniques including pulse modulation adaptive controller (PMAC), direct feedback linear (DFL) control, pattern recognition adaptive controller, two parameter switching control (TPSC), and reinforcement learning controller are used for controlling HVAC systems. In MPC control level, the MPC controller utilizes the system process model to estimate the future state of a system on a prediction horizon. A control vector with the purpose of minimizing a certain cost function (over the prediction horizon) is generated at the end of each iteration.

In this chapter, a review of different types of HVAC control approaches is provided. Comprehensive resources describing the HVAC system control approaches are available. In addition to the many published conference and journal articles, American Society of Heating, Refrigerating and Air-conditioning Engineers (ASHRAE) has also published different handbooks offering a wide range of information on controls ranging from fundamentals to design and application for: building controls [2.1], sequence of operation for common HVAC system [2.2], fundamentals of HVAC control systems [2.3], managing energy and comfort [2.4] and specifying 
direct digital control systems [2.5]. Furthermore, technical documentation of different building energy simulation programs (i.e., transient systems simulation program (TRNSYS) [2.6], HVAC SIMulations PLUS other systems (HVACSIM+) [2.7], and EnergyPlus energy simulation software [2.8]) provide the models of common HVAC system components. A comprehensive listing of building energy simulation software has been maintained by U.S. Department of Energy. This document includes popular simulation tools such as building loads analysis and system thermodynamics (BLAST), esp-r, BSim, and DOE-2. Different toolboxes for simulating the buildings and HVAC system components have also been developed in MATLAB ${ }^{\circledR}$ Simulink ${ }^{\circledR}$ toolkit. SIMBAD toolbox [2.9], international building physics toolbox (IBPT) [2.10], HAM-tools [2.11, 2.12], conventional and renewable energy optimization toolbox (CARNOT) [2.13], and ASTECCA toolkit $[2.14,2.15]$ are some of the available simulation toolboxes.

A few research attempts focusing on some classes of modeling/control approaches have been reported in the literature. Examples include a survey of software used for simulation of HVAC components [2.16, 2.17] and MATLAB ${ }^{\circledR}$ Simulink ${ }^{\circledR}$ used in HVAC systems research [2.18]. While significant progresses have been made in controlling HVAC systems, to the best of the author's knowledge, no recent comprehensive survey of HVAC control systems covering the developed ECSPMs, advanced co-simulator, and the intelligent algorithm used for reducing the MPC computational/optimization time, has been reported in the literature.

\subsection{House and HVAC System Control Approaches}

\subsubsection{Articles Reviewed the HVAC Control Techniques}

Different methods of controlling residential HVAC systems have been reviewed in previous researches. An overview of supervisory and local control of HVAC systems is presented in [2.19]. Naidu and Craig [2.20] reviewed the hard control techniques including gain scheduling, robust control, optimal control, nonlinear and adaptive control and model-based predictive controller (MPC). A comprehensive review of model predictive controllers has been presented by Afram and Sharifi [2.21]. Adaptive control theory and its applications for HVAC systems as hard control techniques are described in $[2.21,2.22]$. The soft control techniques such as fuzzy logic (FL), ANN, and genetic algorithm (GA) have been described and reviewed in [2.23]. Some application 
of ANN and GA for managing energy conservation in HVAC systems have been comprehensively presented in [2.24, 2.25]. Jagdev and Nirmal [2.26] also presented a review of fuzzy modeling and HVAC control systems as soft control techniques. Smart soft control techniques including neuro and genetic-fuzzy are reviewed in [2.27]. Hybrid controllers that are founded by combining hard and soft control techniques have been described in [2.28] survey. A technical review of multiagent control systems (MACs) along with computational intelligence techniques (for energy management) have been presented and reviewed in $[2.24,2.28]$ as other control techniques. The methods used for forecasting the HVAC system loads are reviewed in [2.29, 2.30]. Finally, HVAC simulation approaches, which take into account different models of HVAC components, are presented in [2.31].

\subsubsection{Classification of Process Control Methods}

Classification for HVAC systems control methods is described in this section. The control methods are divided into supervisory control, local control, classical control, hard control, soft control, hybrid control, and other control techniques. Brief details of each method are provided in the following sections.

\subsubsection{Supervisory Level Control}

The set points of the lower level controllers are generated in the supervisory level controllers. In house level, these set points can be determined for HVAC and non-HVAC loads. For example in non-HVAC loads, following set points/profiles could be determined using supervisory level controllers:

- The profile of lighting loads

The supervisory level controller can manage the lighting load based on a scheduled lighting profile. This supervisory level controller can also regulate indoor illuminance according to the outdoor irradiation.

- The profile of specific appliances

Some methodologies can be used to shift the demand of specific appliances (e.g. refrigerators) to off-peak hours using pre heating/cooling process. 
- Charging/discharging levels of battery or PHEV systems during different hours

To prolong the lifetime of the battery bank systems (particularly lead acid batteries), they should be recharged when their State of Charge (SOC) falls below $50 \%$ of their maximum capacity. A supervisory level controller can derive the charging process to charge the batteries when there is surplus solar power or during off-peak hours to minimize the energy cost.

- Solar energy priority on supplying the loads

The supervisory level controllers can use the solar energy as the first priority for supplying the loads in order to minimize both the energy cost and $\mathrm{CO}_{2}$ emission.

- Demand level during the peak hours

Scheduled load profiles can be used by supervisory level controllers to reduce the house internal loads during peak hours. For scheduling the energy in a local area, a supervisory level controller schedules the energy supplied by different sources and the energy consumed by the consumers.

For the HVAC system, following set points/profiles could be determined using supervisory level controllers:

- Pre-cooling/pre-heating profiles

The main objective of using these profiles is shifting the HVAC system load from the peak to midor off-peak hours to minimize the energy cost. House characteristics, outdoor temperature and TOU pricing scheme are the most important parameters that should be considered in the supervisory level controller optimization scheme to optimize the pre-cooling/pre-heating process and related profiles.

- The set point of supply water temperature in radiant floor heating system

The supervisory level controller can reduce the HVAC system cost, by regulating the temperature of supply water in radiant floor heating system. 
- The set point of tank water storage for Domestic Hot Water (DHW) system

The supervisory level controller can decrease the HVAC system cost of regulating the DHW tank temperature during different hours.

- Charging and discharging rate of ice storage system

Thermal storage systems present a great opportunity for building owners to substantially reduce the operating cost by using off-peak electricity to produce chilled water or ice. The supervisory level controllers can take advantage of these systems determining charging and discharging rates.

- Evaporator pressure and damper angle set point generation

The supervisory level controllers can reduce the HVAC system cost of regulating the set points of the damper angles or evaporator pressure.

- The profile of zone temperature set point

This profile can be used to imply the pre-cooling/pre-heating process in order to minimize the HVAC system demand cost.

\subsubsection{Local Level Controls for HVAC System}

The local level controllers usually control variables within the set point ranges determined on supervisory control levels. The controlling process of the HVAC system is complicated because of the complexity of its components. The HVAC system comprises of several heat and mass transfer processes. The zone temperature, humidity and ventilation control are the most prominent applications for the local loop controllers, which directly relate to indoor air quality (IAQ) variables. The following is the list of the most common local loop controller applications in the HVAC systems:

- Zone temperature control

- Supply air temperature control :

- With cooling coils

- With heating coils 
- With reheat coils

- Supply water temperature control on radiant floor heating system

- Supplementary electric heater control in the zone model

- VAV damper position regulation for ZT and ventilation control

- The control of compressor RPM for variable capacity cooling (evaporator pressure control)

- Zone humidity control

\subsubsection{Classical/Conventional Control}

Classical controllers such as on/off, P, PI, and PID controllers are the most commonly used control techniques. The on/off controllers use upper/lower thresholds to regulate HVAC system within given boundaries. However, P, PI, and PID controllers modulate the controlled variable and using error dynamics to achieve accurate control of the HVAC performance.

Classical controllers have been used in many studies for controlling HVAC system. For example, $[2.32,2.33]$ used classical controllers for dynamic control of cooling coil units. Classical controllers have been used for controlling the zone temperature in [2.34, 2.35] surveys. Supply air temperature control [2.36, 2.37], supply air pressure control [2.33, 2.38], damper gap rate control $[2.33,2.39]$, variable air volume unit temperature control [2.40], heater control [2.33], and evaporator supply heat control [2.40] are all designed based on classical controllers. However, these studies have mostly focused on PID controllers using auto-tuning and optimal tuning methods.

Although the on/off controller is the most intuitive and one of the easiest controllers to implement, it cannot control the moving processes that have time delays. Many processes of HVAC and house thermal systems have high thermal inertia. As a result, the processes that are controlled with an on/off controller experience large oscillation from the set points. On the other hand, the PID controllers produce promising results; however, tuning the parameters of controller is cumbersome. Furthermore, the performance of the controller declines if tuning conditions vary from the operating conditions. In addition, auto-tuning and retuning approaches of the PID controller can be time-consuming [2.41]. Based on [2.42] survey, auto-tuning might be unacceptable, in certain applications, because of its uninvited nature relative to the normal operation. 


\subsubsection{Hard Control}

Hard controllers are designed based on a theory for control system and consists of nonlinear control, robust control, optimal control, gain-scheduling control, and MPC control. Nonlinear controllers take advantage of both nonlinear process models and control techniques to find the system's stable states and derive the control law to lead the system toward a stable state. The robust control works well under varying disturbances imposed to the system and also with changes in the parameters. In gain scheduling control technique, piece wise linear regions are generated from a nonlinear system and the PID controller's gains are determined in each region. The optimal control algorithm will find a solution for an optimization problem based on an appropriate cost function in form of minimization of demand and/or energy cost.

Hard controllers have broad applications in controlling HVAC systems. For example, in [2.43], two PI controllers were tuned to meet high/low heat demand conditions in a radiator-based HVAC system. For supply air pressure control, a PI controller is used in [2.38]. This controller gains based on the error between the measured supply air pressure and the defined set points. For designing nonlinear controllers in hard control techniques, the control laws can be derived using feedback linearization, Lyapunov's stability theory, and adaptive control methods. The control laws using for driving nonlinear systems toward stable states while achieving the control goals. Nonlinear controllers have a wide range of applications in regulating the HVAC system performance. For example, a nonlinear controller has been applied in an air handling unit (AHU) control [2.44], control of greenhouse gas emission [2.45], and water to air heat exchanger cross flow control $[2.46]$.

The purpose of designing a robust control is to develop a controller that performs well under timevarying disturbances as well as variation in parameters. Supply air temperature control [2.47], zone temperature control [2.48] and supply airflow rate control [2.47] are examples of robust control techniques. In optimal control algorithms like MPC, an optimization problem is solved to minimize a certain cost function. The objectives of optimization processes in HVAC systems are mainly minimization of energy demand and energy cost without sacrificing thermal comfort.

Model-based predictive controllers (MPCs) are considered an advanced type of hard controllers. MPCs consider the future state of the model into optimization scheme. In comparison with the conventional (on/off, PI and PID) controllers and other types of hard controllers, MPC is much more powerful. MPC provides an anticipatory control mechanism by predicting the future state of 
the system based on the estimate of components behavior and produces a constrained control matrix, which drives the system towards the optimum state. MPC finds the optimal solution for different optimization problems by using a cost function (i.e., energy consumption). The operating mechanism of MPC controllers will be discussed later in Section 2.2.3.

\subsubsection{Soft Control}

Soft control techniques comprise of fuzzy logic (FL) [2.49, 2.50], artificial neural network (ANN) $[2.51,2.52]$, and GA based controllers. New control techniques can be established by incorporating these techniques into digital controllers. In a fuzzy logic controller, the control actions are determined based on "if-then-else" statements. FL can be used for auto-tuning of PID controller gains where PID controls represent local scope of the control, and the FL supervisor is usually used to optimize the system response on the global scale. The fuzzy logic supervisor also behaves as an arbiter to resolve conflicting objectives of local level controllers. This can be conducted by prioritizing specific controllers over others to achieve the common goals like reducing energy consumption and/or maintaining thermal comfort. Alternatively, the fuzzy logic controller can be implemented in both the supervisory and the local levels of control. For example, in [2.53] a thermal comfort controller designed using predicted mean vote (PMV) method to control humidity, temperature, and air velocity of an AHU. Another example of fuzzy logic controller is the development of a three-level hierarchical supervisory controller designed to regulate/manage the operating modes of the air and water subsystems and to set the set points for the lower level controllers [2.54].

The artificial neural networks are trained based on the system performance data to fit a nonlinear mathematical model with the measured data. The concept is to use black box modeling techniques that do not require an understanding of the process underlying physics. The ANNs have broad applications in feed-forward control, and can be trained using the controller input/output in order to be replaced with a conventional/classical controller in that application. Examples of neural networks design include a zone temperature controller using PMV method [2.55], controlling the fan speed of an air cooled chiller [2.56], and the optimization of air conditioning setback times based on the outdoor temperature [2.57]. 
A comprehensive knowledge of the plant operation is required for running the fuzzy logic controllers. Comprehensive operational databank is required for training a neural network. This data may not be practically available for some systems.

\subsubsection{Hybrid Control}

Hybrid controllers are founded by combination of hard and soft control techniques. Soft and hard control techniques supplement each other, and when combined they can solve problems that may not be solved by either of them. Several controllers for HVAC systems including adaptive-neuro control [2.52], quasi-adaptive fuzzy control [2.58], and fuzzy-PID control [2.50], have been presented in the literature. Hybrid controllers consist of soft control techniques including ANN at higher levels and hard control techniques like adaptive controllers at the lower control levels. For example, in [2.38] a fuzzy self-tuning PI controller was used for supply air pressure control. In [2.58], the consumption of a convector-radiator heating system was controlled using a quasiadaptive fuzzy controller. Although hybrid controllers benefit from both soft and hard control techniques, they also inherit problems. For example, designing and training a soft controller requires user expertise and big amounts of data. On the other hand, a hard controller may be difficult to design using the wide range of performance conditions often observed in the typical HVAC systems.

\subsubsection{Other Control Techniques}

The other control techniques including pulse modulation adaptive controller (PMAC), direct feedback linear (DFL) control, pattern recognition adaptive controller, and two parameter switching control (TPSC) [2.55] have been developed for controlling HVAC systems in the literature.

Figure 2-1 illustrates the flowcharts of different controlling techniques described in Section 2.2.2 and used for constructing various system controllers for HVAC systems from the classical simple ones to the developed and novel control techniques. 


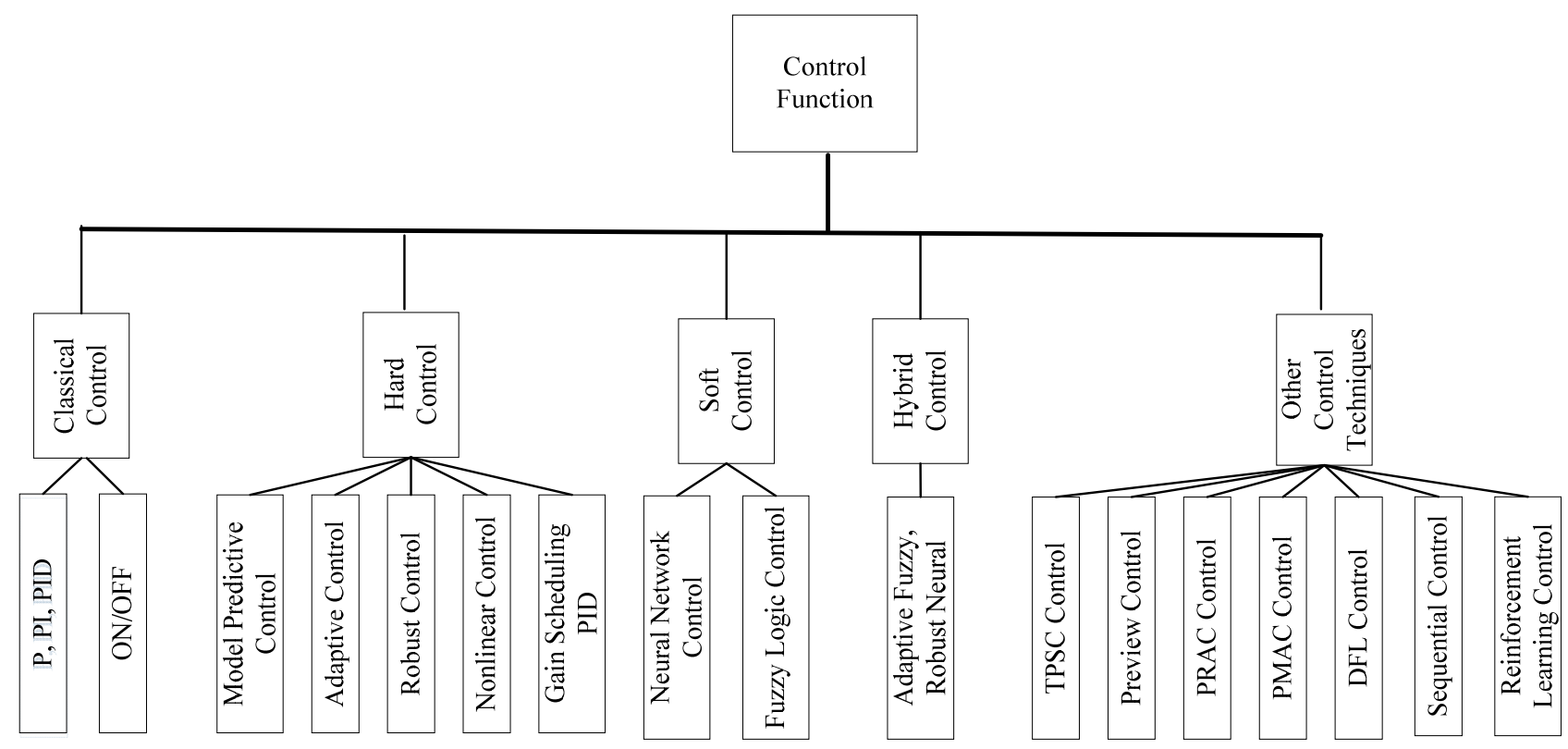

Figure 2-1: Different controlling techniques and functions used for constructing various system controllers for HVAC systems

\subsubsection{Summary}

Considering the technical requirements and characteristics of HVAC systems, the MPC controllers offer significant advantages. In HVAC systems, many processes are slow moving having time delays and are affected by temporal external and internal disturbances. Under the effects of these parameters, HVAC systems experience a wide range of operating/performance conditions. In addition, HVAC system actuators exhibit range and rate limit constraints. In many provinces, energy has a variable pricing scheme. Considering all these challenges, the ideal controller should be capable of handling: time-varying disturbances, actuator constraints, wide operating conditions, and variable pricing scheme. Apparently, many control systems suffer from different shortcomings when it comes to controlling HVAC systems. For instance, the classical/conventional controllers require manual tuning and perform too aggressively or sluggishly outside of their tuning band. Rigorous mathematical analysis is required for the hard controllers and soft control needs significant amounts of data for reinforcement and training. Furthermore, learning techniques require remarkably long time for training and tuning that renders them impractical for industrial 
implementations. Alternatively, MPC controllers provide solutions for many of the aforementioned problems/difficulties, and therefore are the focus of this research.

\subsubsection{Model Predictive Control (MPC)}

A comprehensive review of different MPC techniques is presented in this section. To show the advantages of this control method, its efficiency is compared with the efficiency of other techniques used in other projects.

An MPC controller utilizes the system process model to estimate the future state of a system on a prediction horizon. A control vector with the purpose of minimizing a certain cost function (over the prediction horizon) is generated at the end of each iteration. In order to generate the control vector which drives the system toward the optimum condition at each sampling instant, an optimization problem should be solved to minimize the cost function in the presence of constraints and indoor/outdoor disturbances. Since model inputs/outputs and indoor/outdoor disturbances will change over time, at any sampling time only the first element of the generated optimal control vector is selected and applied into the system while the rest of the elements are discarded. The cost function could be formed based on control effort, tracking error, energy and demand cost, power consumption or a combination of these functions, which should be minimized at each time instant. Plant system constraints and limitations can be determined based on the actuators' rates, range limits, and the boundaries of the system. For example, in an HVAC system, zone temperature upper/lower thresholds, DHW tanks temperature limitation, flux limitation based on the area, supply/return air flow-rate limits, damper position and speed ranges are some of the constrains that should be considered during optimization. Some of the internal and external disturbances affecting the process system are weather, internal gains due to solar passive effect, thermal mass, appliances, lighting and occupant behaviors. The predicted impacts of these disturbances are considered in the model while generating the control vector. The MPC model performance depends on various factors such as plant and process model details, optimization scheme and method, prediction and control horizons, disturbances effects, constraints and the cost/objective function, employed in the MPC models.

The MPC models have the potential to be used in both the supervisory and control levels. 


\subsubsection{Comparing MPC with other Control Technique and Approaches}

The following metrics are usually used to compare the performance of different controllers:

- Cost/energy savings $[2.59,2.60]$

Two of the most important parameters, which prove the advantage of different model controllers, are the energy cost of the whole system and the energy or demand saved using each model controller.

- The capability of shifting the peak load [2.59]

One of the most important features, which directly affect the energy cost of a system, is its ability to shift the load from peak to off- or even mid-peak hours. This feature can be used to compare the effectiveness of various model controllers.

- Controlled variable within the boundaries [2.61, 2.62]

The optimization scheme used in various controllers should assure the process system constrains. This important ability can be used to compare the performance of different controllers. The following factors can also be used to examine the pros and cons of various process system controllers:

- Set-point fluctuations (regulation)

- Efficiency and Coefficient of Performance (COP) improvement

- Robustness to disturbances

- Transient response (rise time, settling time, peak time)

- Steady state response (offset error)

- Indoor air quality

Based on the result of the previous surveys [2.63, 2.64, 2.21], higher energy and cost saving could be obtained by using a properly developed MPC controller. Furthermore, the MPC controller has 
the potential to shift the peak load to off/mid peak hours considering variable pricing scheme in order to minimize the energy cost. With this mechanism, more energy will be consumed when the cost is low, i.e., during the off/mid peak. In any house, one of the most important issues, which should be controlled perfectly, is the Indoor Air Quality (IAQ). IAQ depends on the zone temperature, humidity, and the level of $\mathrm{CO}_{2}$. These variables should be kept within desirable limits in order to ensure the acceptable IAQ. These variables could be optimally regulated using a properly designed MPC controller.

Conventional/classical controllers are tuned to work at specific loads and set points. As a result, their response to operational condition, which is different from tuning conditions, is usually too aggressive or lumpish. In contrast, considering the MPC ability to integrate the new information and disturbance prediction into the control decision system at each sampling horizon, the response of this transient system is better than the conventional controllers' response. Generally, the classical controllers regulate the system tightly around the set points, reducing fluctuation. However, the MPC controllers not only have the potential to regulate the process around a set point, they also keep the controlled process within a specific boundary in order to minimize the cost function.

The abovementioned comparison, proves the superiority of the MPC controller over classical/conventional control techniques, i.e., on/off, P, PI and PID. Researchers mostly use one or a couple of performance comparison metrics to evaluate their proposed controller's performance against others basic methods. Ma et al. [2.64] used an MPC controller to control the zone temperature. Taking advantage of the MPC model, the peak load was shifted to off-peak hours to flatten the on-peak power profile. The result of this research was compared with the result of different conventional controllers and SPMs. MPC allows greater cost savings (28\%) compared to baseline night-setup strategy (0\%), linear (17\%) and step-up (24\%) SPMs. Prívara and colleagues [2.65] described an MPC model for controlling room temperature. MPC used 29\% less energy than a finely-tuned controller that fed the weather prediction data into the model to ensure the same level of thermal comfort. Moroşan et al. [2.66] precisely managed zone temperature during occupancy period. In comparison with PI controller, a 36.7\% increase in thermal comfort was achieved by using centralized and distributed MPC controllers. Using these controllers, the energy consumption was also reduced by an additional $13.4 \%$. 
According to the literature review, it is concluded that the MPC Controller is one of the best solutions for controlling the energy consumption of a house, especially of its HVAC system. Hence, in this thesis more attention is paid to MPC approaches.

\subsubsection{Comparing Various MPC Methods and Approaches}

A model-based predictive controller is constructed of different components including system process model, constraints, disturbances, cost/objective function, optimization scheme, and control horizon. Figure 2-2 illustrates a sample of a demand side model-based predictive controller consisting of the abovementioned parts. It should be noted that the appropriate choice of each part directly affects the performance of the MPC model. Various MPC approaches can be compared considering the type of controller, building/HVAC system, the processes they are controlling, energy conservation strategy planning used, prediction/control horizon, model used to process dynamic simulation, disturbances/constrain considered, time step length, cost/objective function used, and the optimization problem solved. In the following sections, these properties would be described and compared.

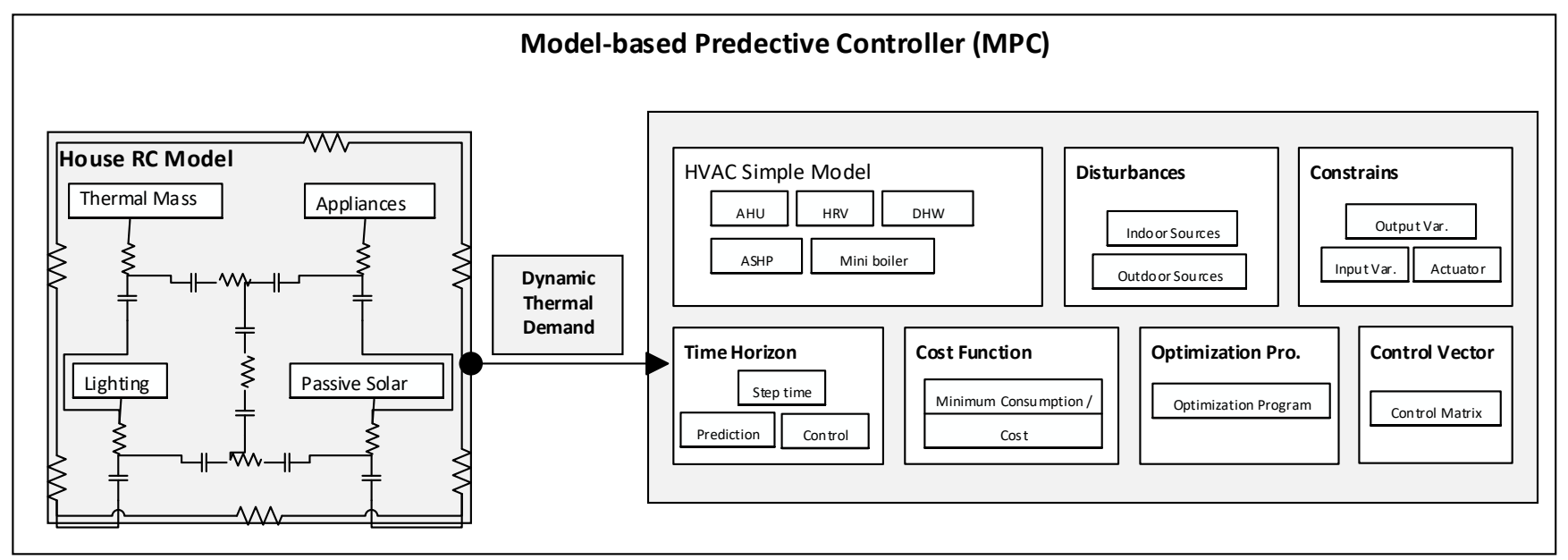

Figure 2-2: Different components of a HVAC system MPC controller 


\subsection{Control Type and Configuration}

The MPC configurations can be classified into different categories, and each category itself can be divided into cascaded, hierarchical, centralized, de-centralized, and distributed structures. The MPC can be used as a lower (local) and/or higher (supervisory) level controllers. The controllers can be used in cascaded or hierarchical design to manage both fast and slow moving disturbances. In a hierarchical structure, the MPC supervisor controller can be combined with the classical local loop PID controllers [2.67]. Wallace et al. [2.68] combined the MPC controller with a cascaded PI controller. The MPC model can also be used in hierarchical control in both upper and lower levels [2.69] and in cascaded configuration in both inner and outer loops [2.70].

Centralized, de-centralized, and distributed MPC can be used for controlling multi-zone buildings [2.71]. The de-centralized control can use the same local controller for each zone separately without any consideration of zones thermal coupling. The centralized controllers, on the other hand, consider the inputs, outputs, occupancy, and thermal coupling for all zones simultaneously. As a result, they are able to track each zone set point despite various zone temperature set points and occupancy periods. However, a centralized MPC controller results in a higher computational time as well as lower reliability since any technical problem in the central controller disables the HVAC system in the entire building. Furthermore, this system can not be used in large buildings since implementing the controller will require more MIMO models and a significant amount of computing power. The simulation result in [2.71] showed that the performance of a distributed controller is comparable with that of a centralized controller. Furthermore, similar temperature regulation and energy cost savings were gained using distributed controllers while their computational cost was lower than the cost of decentralized controllers.

\subsection{Controlled Process}

A house and its HVAC system each contains many components and subsystems, which can be controlled independently. The most important variables that directly affect the thermal comfort are zone temperature, humidity and ventilation.

The set points considered for pressure, flow rate, and temperature (in the water and refrigerant loops) can be considered as controlled variables that are regulated by pumps, fans, compressor,

valves and boiler. Similarly, the flow rate, temperature, and pressure in the air loop can be also considered as controlled variables that are controlled by the fans, dampers and heating/cooling 
water flow-rate valves. The valve and damper positions, compressor speed, consumption rate, fan speed, boiler fuel, and pump speed can be all considered as manipulated variables. Following, the examples of literature that controlled HVAC system operating mechanisms for regulating the zone temperature and minimizing the HVAC system energy cost are presented. The thermal comfort was optimized in [2.72] using a predicted mean vote (PMV) controller. Zone temperature was controlled by different MPC models in different studies [2.64, 2.66, 2.73, 2.74, 2.75]. The HVAC system energy consumption was minimized in [2.76].

\subsection{Building Single/Multi Zones MPC Structure}

MPC controllers have been implemented in a variety of building HVAC systems. For example, a MPC controller was applied in: a single-story office building to control the zone temperature [2.77], in damper process and zone temperature control of a single-zone VAV system [2.78], and in air temperature control of a large factory constant air volume (CAV) system [2.79]. HVAC systems can serve both single and multi zone buildings. In a single zone building, the set points of indoor air quality variables are the same in all rooms/areas. However, in a multi zone building, the set points of the different zones are controlled by the user(s). Therefore, designing a controller for a single zone building is easier since simplifications can be used for thermal properties and geometric of the building because of poor insulation between the zones. However, in this simplification, coupling cannot be ignored and must be modeled appropriately to control the zone temperature, humidity, and air quality factors accurately. In this case, this strategy leads to more complex MIMO controllers. Various MPC strategies have been implemented in both single zone and multi zone buildings. Examples of such building include test room [2.71, 2.80, 2.81], single story office building [2.77], multi-story office building [2.82], small studio apartment [2.83], factory building [2.79], and large university building [2.84, 2.85, 2.86].

\subsection{External/Internal Disturbances and Prediction Basis}

The MPC model needs to predict the system's future state based on the estimation of the internal and external disturbances affecting the system. Occupancy (as an internal heat gain source), passive solar energy, lighting system, and appliances are some sources of internal disturbances estimated and considered in [2.64, 2.87]. The external disturbances like outdoor temperature, wind speed and solar irradiations on different orientations can be predicted using the short-term 
deterministic weather forecast dataset. It should be noted that HVAC operation is significantly affected by outdoor disturbances. For example in [2.88], the effects of uncertainties in weather forecast system were investigated in thermal comfort violations and energy consumption of an HVAC system. In this study, the zone temperature, regulated using a rule-based control (RBC), was compared with those of stochastic MPC (SMPC) and deterministic MPC (DMPC). A theoretical benchmark, known as performance bound (PB), was also used in this study to investigate the theoretical saving potentials of DMPC, SMPC, and RBC methods. For computation of $\mathrm{PB}$, it was considered that the weather forecast had no uncertainty and was $100 \%$ accurate. With this assumption, the DMPC maximum savings potential was calculated. Apart from the external and internal disturbances discussed above, there are other internal operational disturbances like water inlet temperatures and variable air mass flow rates in the AHU [2.79] and coupling between adjacent zones [2.71] that act as disturbances in a MPC control system. Some studies used simulated disturbances (e.g., heating at an unknown rate [2.89] and random noise [2.90]) in their proof of concept.

One of the basic requirements of MPC controller is to predict the system future states. When there is no accurate process model, HVAC system developers use reference signal future value [2.91], the tracking error prediction [2.73], and the historical values of the control signals [2.74] to predict the future states of the system using an MPC controller. An MPC controller that uses a system forecast, that generated by abovementioned models, can outperform other techniques that do not use weather forecasting system/information.

\subsection{Process Model Used for Controller Development and System Dynamics Simulation}

As mentioned earlier, the MPC controller can use physics based (also known as forward or analytical first principle models), data driven (also known as inverse or black box models), and/or gray box process models (as the combination of white and black box models) to predict the future state of a system. Physics-based models are founded based on the knowledge of the physical process and parameters. Parameters can be determined based on manufacturer documentations and/or parameter estimation techniques (i.e., least square method) using measured process data. Physics-based models have been previously developed for mixing boxes [2.92], AHUs [2.93, 2.94], fans [2.95], and zones [2.96-2.97]. The physics-based models of thermal processes are similar to electrical RC networks. For simplicity, these physics-based models use lumped thermal 
capacitance/resistance instead of distributed thermal capacitance and resistance. This strategy leads to simple dynamic (first-order) models that denote the thermal process.

Data-driven/black box models fit linear and nonlinear mathematical functions based on the measured/historical data. Examples of black box models include FL [2.98, 2.99], ANN [2.51, 2.100], support vector machine (SVM) [2.101], statistical models (e.g., autoregressive (AR), first and second order time delay models [2.102, 2.103], autoregressive moving average (ARMA), autoregressive with exogenous (ARX), autoregressive moving average exogenous (ARMAX), finite impulse response (FIR), box jenkins (BJ) models [2.104], and output error (OE). The accuracy of black box models is higher than that of physics-based/white box models; however, black box models suffer from generalization abilities.

In addition to white, black and gray box models, MPCs can utilize comprehensive process models by taking advantage of house energy simulators such as TRNSYS [2.59], EnergyPlus [2.64, 2.59], and Simulink [2.66, 2.74]. These models provide very accurate results useful for performance analysis and optimization process. However, in order to solve the optimization problem, a process model should be run several times based on different constrained control matrices to find the optimum state of a system. Reprocessing the house energy simulator leads to unacceptably long optimization time. As a result, these models are not usually beneficial to the MPC process model. Hence, process models should be built based on simpler physics based and data driven approaches with reasonable simplicity and accuracy and short reprocessing time.

To develop white, black and gray box models with high level of accuracy, the data should have low noise, high accuracy, and proper temporal resolution to capture the process/plant dynamics correctly. For fast moving processes and variables in HVAC systems, like water and airflow rate measurements, the higher sampling rate should be selected compared with that of slow moving processes such as air and water temperatures. For controlling the HVAC system, data sampled in one-minute intervals are appropriate for fast moving processes. However, for slow-moving processes hourly data may be appropriate. Averaging and median filters can be applied for removing quantization noise and spike noises, respectively [2.105]. The data should also cover a wide range of HVAC system operating conditions during different weather conditions and occupancy behavior throughout the year. Considering changes in the building/house and HVAC parameters over time, the prediction of model maybe deviate from the actual output. To tackle this issue, the process models should be updated over time with new values of parameters. The model 
accuracy can be increased by clustering the data into similar outdoor weather conditions or different seasons [2.106]. Multiple models can be trained based on these data clusters in order to select the appropriate model. After developing the model, model validation is required to verify its accuracy. Model validation can be performed by comparing the outputs of model with measurements, results of other modeling software or with analytical solutions of a known problem [2.107].

Performance metrics have been previously defined in the literature to compare the prediction results of different models and to calculate their deviations from the measured data. Models can be compared using: absolute error $(A E)$, maximum absolute error $\left(M A X_{A E}\right)$, mean bias error $(M B E)$, mean absolute error (MAE), mean squared error (MSE), mean absolute percentage error (MAPE), absolute percentage error (APE), standard deviation of absolute error (Std $A E)$, standard deviation of absolute percentage error (StdA $A_{P E}$ ), root mean square error (RMSE), coefficient of determination $(D)$, coefficient of variation $(C V)$, goodness of fit $(G)$, mean absolute relative error (MARE), relative mean error $(R M E)$, coefficient of multiple determination $\left(R^{2}\right)$, and correlation coefficient (CC) $[2.51,2.99,2.106,2.108,2.109,2.110]$.

One example is reviewed from the literature and described here to obtain insight into model development and its further applications in MPC. In [2.83], physics-based models were developed for designing a MPC to control the zone temperature inside a studio apartment. The study primarily focused on parameter identification of models for zone temperature, control signals, and HVAC energy consumption. The thermal conductance and capacitance of air along with capacitance of structural nodes of the building were the estimated parameters in this study. Two different types of parameter estimation algorithms were presented/applied in this study to find estimates of the conductance and capacitance of the building. To regulate the zone temperature (based on models with estimated parameters) a rule-based MPC controller was applied in this study.

In process modeling, it should be noted that based on the simplicity that linear models offer in control development, some MPC designers attempt to linearize the obtained process models using feedback linearization [2.79] and Jacobian linearization [2.111]. Linear models can also be generated using system identification techniques [2.112] and prediction error method [2.89]. 


\subsection{Prediction Horizon, Control Horizon and Time Step/Instant}

Prediction horizon, control horizon, and time instant are very important parameters and play significant roles in performance, operation and outputs of the designed MPC controllers. The prediction horizon refers to the length of time in which MPC outputs are computed, whereas the control horizon refers to the length of time in which the control signals are computed. The time step or control sampling time is the time that during which the control signals remains unchanged. Typically, for slow moving processes in the HVAC systems the prediction horizon range can be considered between $2 \mathrm{~h}$ and $48 \mathrm{~h}$, the control horizon range can be considered between $2 \mathrm{~h}$ and 24 $\mathrm{h}$, and the time step is between 1-min and $1 \mathrm{~h}[2.79,2.84,2.112]$. The control horizon should be smaller than or even equal to the prediction horizon. The selected horizons depend on the dynamics of controlled processes. Using very small prediction horizon or a short sampling time for temperature processes could result in controller performance degradation due to delays in the these processes. However, using a longer prediction horizon could result in unacceptably long computation time without any further benefits [2.81]. When applied to fast moving dynamic processes such as superheat temperature control and compressor pressure, the prediction horizon and control horizon can be shrunk to only a few seconds only [2.89]. In some specific applications, a time-variable/temporal horizon is also employed. For example, in [2.77], for optimizing energy consumption over a $24 \mathrm{~h}$ period, a shrinking horizon scheme was implemented in which the prediction horizon reduced when the time progressed towards the end of the day.

\subsection{Constraints}

The MPC controllers are also known as constrained controllers because of their ability in finding solutions that do not violate the constraint placed in the inputs, outputs, and actuators. There are different types of constraints including equality constrains such as capacity limits of components and inequality constrains such as actuator rate and range limits. In addition to defining constraints for actuators, controlled variables can be also limited to range and rate constraints. For example, in order to maintain thermal comfort, the zone temperature may not be allowed to deviate with more than a certain amount per unit time. Furthermore, the zone temperature should be maintained within a standard certain band. To understand different types of constraints in MPC development, following examples are described from the literature. In the case of temperature process control, 
the supply air temperature [2.79] and supply airflow rate [2.78] were constrained to only operate in given ranges. In the case of controlling the room temperature in [2.80], limits were defined in indoor temperature and in the supply heat flux.

\subsection{Cost/Objective Function}

The cost functions are defined based on the desired behaviors of the designed system. The cost functions also serve to stabilize the system. In should be noted that in systems with slow dynamics/processes (i.e., temperature processes), stability is not an issue, and designer can choose any form of cost functions. The cost functions in HVAC systems also describe the performance targets such as the energy consumption minimization and the thermal comfort maximization. In a house and its HVAC system energy model, cost/objective function represents the function which would be optimized while keeping the thermal comfort at standard levels. In this thesis, optimizing the thermal comfort and the usage of renewable sources, minimizing the energy consumption, energy cost and the usage of battery bank systems are the different competing objectives, which should be achieved. To this end, varying weight factors should be incorporated into the cost/objective function. The following cost functions are typically used in a building and its HVAC controller optimization schemes:

- $\quad$ Energy/demand and operating cost $[2.59,2.64]$

As mentioned before, energy and demand costs are two important issues, which should be minimized through proper optimization.

- Integrated power consumption [2.75]

The HVAC system consists of different components with different tasks. Each component has its own consumption. On the other hand, a residential house contains various electric devices. The combination of these loads complicates the process system. In this case, optimization of integrated power consumption with its all complexities is one of the objective functions defined in MPC model optimization schemes.

- The sum of solar generation [2.91] 
The maximum usage of solar energy in supplying the loads is one of the main objectives of supervisory/administrative MPC model optimization schemes.

- The percentage/usage of battery bank/PHEV storage systems for supplying the loads [2.91]

The contribution of storage systems in supplying the loads is another objective of MPC controller. This approach will prolong the battery bank lifetime and minimize the energy cost. The tracking error function is another objective considered in MPC optimization:

- Tracking error quadratic cost function [2.65]

- Sum of tracking error [2.75]

The daily dynamic energy cost function can also be defined considering the different weights on the energy consumption function. These weights are changed based on the energy pricing scheme (TOU) during the day.

\subsection{Optimization Problem}

After constructing and formulating the system models, constraints, disturbance models, and also cost functions, MPC controllers solve a constrained optimization scheme to compute/determine the optimum control vector. Since gradient-based techniques, which are mainly developed to work with continuous functions, may not be able to find the global optimum of the function, a classification of optimization methods was previously proposed in the literature. For example, in [2.113, 2.114], a classification of linear/nonlinear optimization methods is given for HVAC control. Freire, Oliveira, and Mendes [2.72] presented four different cost functions and optimization schemes. Thermal comfort (including zone temperature and relative humidity) and energy consumption are the decision variables optimized in this project by sum of tracking error and control effort functions. Tazvinga et al. [2.91] developed a multi-objective optimization model. The model optimizes the house energy dispatch by minimizing operation cost and maximizing utilization of renewable energy while considering the battery bank lifetime. The optimization problem is solved taking advantage of "quadprog" function in Matlab. Ahmad et al. [2.115] used a typical MPC objective function for optimization. In this project, time varying 
weighting factors (which changes based on the energy price), occupancy profile and weather prediction are considered during optimization. In Ma et al. [2.64], "Linprog" Matlab function is used to minimize the demand and energy cost using a linear program solved by the weighted sum of tracking error function. Matlab MPC toolbox is used in [2.116] to optimize the formulated objective function. Quadratic programing is used to optimize zone temperature using the tracking error and control effort in [2.73]. In [2.74] a constrained nonlinear function is developed and solved by using 'fmincon' Matlab function in order to minimize the deviation of indoor temperature. The integrated heat power consumption rate is optimized on a MPC model presented in [2.75] using dynamic programing algorithm.

The principle of mixed integer programming (MIP) was used by Gregor and colleagues [2.116] to minimize the power consumption of a cooling plant. Elliott [2.117] used Wolfe-Dantzig quadratic programming $(\mathrm{QP})$ algorithm to solve the optimization problem using Matlab MPC toolbox QPDANTZ function. Xu and colleagues [2.65] used Iterative Dynamic Programming (IDP) to solve the convex quadratic optimization function. Yuan and Perez [2.60] formulated constrained (convex) optimization problem to minimize the tracking error and control effort functions. Quasi newton and dynamic programing techniques were used by Gregor and colleagues [2.59] to optimize the passive and active storage, respectively, using Matlab optimization toolbox. The operating cost was minimized using TOU electricity pricing scheme and natural gas fixed prices.

\subsection{HVAC System Energy Conservation Strategy Planning Models}

Energy can be conserved by employing different energy conservation strategy planning models including thermal storage in the building/house mass [2.77], passive solar gains [2.112], night setbacks, pre-cooling/pre-heating during off-peak periods and set point reduction during peak hours [2.119], temperature reset during unoccupied hours [2.52], optimum start and stop times [2.120], floor heating mass [2.112], thermal storage in tank water [2.81, 2.82], economizer cycle control [2.121] and ventilation control [2.122]. Some of these conservative strategies can be implemented together using MPC controller to maximize energy saving or minimize energy demand. The cost/objective function(s) of a predictive controller can be constructed using energy conservation strategies. For example, a cost function can be founded to shift the HVAC load from peak hours to off-peak hours to minimize energy consumption during peak hours. However, based on outdoor disturbances, the peak load shifting does not always lead to lower energy 
consumptions, it may also result in lower operating costs with the presence of a variable energy pricing scheme. It has been reported in [2.81] that thermal energy storage, as one of the energy conservation strategy planning models, results in a significant reduction in HVAC operating cost. Even a simple non-predictive control strategy like chiller priority strategy that uses thermal storage, resulted in greater cost savings when compare with a system without thermal storage. In [2.123], it has been shown that passive storage in building/house mass resulted in the highest savings when the buildings thermal mass material increased. As a result, passive thermal energy storage during the period in which energy price is less expensive can be considered as a powerful method for increasing energy cost saving in residential houses. Taking advantage of novel strategies like dual fuel switching systems to switch the HVAC system to a fuel with lower timevaried energy price is another powerful control strategy that can be employed in the HVAC system.

\subsection{Conclusion}

In Chapter 2, the general techniques used for controlling HVAC systems including supervisory control, local control, classical control, hard control, soft control, hybrid control, other control and MPC techniques were reviewed. In comparison with most HVAC control techniques, MPC controllers generally have superior performance in terms of better transient response, lower energy consumption, consistent performance under varying conditions, and robustness to disturbances.

The structures, foundation and the parameters affecting the performance of different types of MPC controllers were described in this chapter. The different energy conservation strategy planning models employed in residential HVAC systems were also reviewed. The important points of HVAC MPC control development can be summarized as follows:

- Weather forecasting, indoor/outdoor disturbance predictions, and accuracy of the model affect the performance of MPC controller. Updated information including measured weather variables (ambient temperature, wind speed, humidity and solar flux) should be incorporated into the MPC at each sampling time to improve controller performance.

- Most MPC models use discrete linear equation of the system obtained by either creating linear ARX models from measured data or linearizing the state-space models around a specific equilibrium point. The system identification techniques are used for deriving simple linear 
models from comprehensive models developed in house thermal energy simulators including TRNSYS and EnergyPlus. In addition, MPC can be incorporated in TRNSYS, EnergyPlus, and Simulink comprehensive models to simulate control performance for a real building/houses when no comprehensive/complex optimization scheme is required.

- Selection of the prediction/control horizons and sampling time affects computational cost, accuracy, and response time of MPC controller. Cascade and/or hierarchical MPCs are designed to handle both slow and fast moving disturbances. Supervisory-level MPC controller is used for controlling slow dynamics which operates with using longer time horizons and slower sampling times. Lower/local-level controller is used for controlling the fast moving disturbances that operates on a shorter horizons and faster sampling times.

- Even a very basic form of MPC controller (with simple disturbances, simple linear constraints and load forecasting models) outperforms the conventional control approaches that do not include any built-in predictive controllers/algorithms.

- Energy conservation strategy planning models can be integrated into MPC design. Thermal energy storage presents excellent opportunities for peak load shifting and reducing the HVAC operating costs. The MPC with thermal energy storage platform outperforms controllers that do not use thermal energy storage. Buildings with large thermal mass material could utilize passive thermal storage by pre-heating or pre-cooling the building/house during the off-peak hours. In specific outdoor disturbances, the use of thermal energy storage may result in higher energy consumption. However, lower energy costs may be achieved because of the variable time of use pricing scheme through the day.

Many MPC control techniques, structures, process models, and internal parameters reviewed in this chapter will be used in the next three chapters for both establishing novel energy conservation strategy planning models and advanced co-simulator as the first MPC controller and developing a novel intelligent algorithm for reducing the MPC computational/optimization time in the second MPC controller for TRCA-ASH House A. The performance of MPC models will be compared with that of the conventional/classical controllers to show the effectiveness of the developed MPC models. 


\section{Chapter 3: Development of First MPC Controller Using Matlab-TRNSYS Co- simulator for Applying Predictive Strategy Planning Models}

The development of first MPC controller using Matlab-TRNSYS Co-simulator is discussed in this chapter. This chapter covers the contents of the first journal paper that was published in the Journal of Energy and Buildings.

In the first MPC controller, a comprehensive process/plant model has been used for house thermal energy and HVAC system using TRNSYS program. Building energy simulators such as TRNSYS, EnergyPlus, and esp-r offer an excellent opportunity for detailed design of house thermal model and its Heating, Ventilating, and Air Conditioning (HVAC) system and provide very accurate simulation results useful for performance analysis and optimization process. In contrast, these energy simulators do not include sub-models of advanced devices/strategies for control of HVAC system operation and suffer from poor control mechanism. In addition to the lack of an advanced controller, they inherently offer no mechanism for estimating the future state of their process models based on forecast weather dataset. Hence, no predictive controller can be designed and implemented within these simulators. This chapter discusses the development of first MPC controller in order to control/manage a TRNSYS program, which was previously developed and calibrated based on the characteristics of a real case study house. This MPC controller investigates the effectiveness of novel predictive strategy planning models including Load Shifting (LSH), Smart Dual Fuel Switching System (SDFSS), and LSHSDFSS, as the integration of fuel switching and load shifting strategy planning models on 24 hours ahead energy cost saving of the case study house HVAC system. Simulation results of three consecutive sample days indicate that SDFSS could bring significant energy cost saving. However, LSH and LSHSDFSS effectiveness is sensitive to outdoor temperature.

\subsection{Introduction}

A high percentage of urban dwellings consists of residential houses (RHs). Hence, RHs can play significant roles in managing the network energy system. In order to investigate the effect of RHs on network energy system, different kinds of research have been previously conducted. For example, Mathew et al. [3.1] developed an internet-based distributed system utilizing distributed 
load shifting strategy to manage the community energy system. Liu and colleagues developed a constrained demand-side management system considering peak-to-average ratio and consumers' preferences in optimization routine for managing the energy systems of different residential houses [3.2]. Radhakrishnan and Selvan used load scheduling technique along with decentralization of power generation in various residential buildings to manage the network energy system [3-3]. In addition to their extensiveness, residential houses inherently have the potential for storing thermal energy, therefore, they present a great opportunity for managing/controlling electricity demand during peak hours utilizing various control techniques including advanced control system design [3.4] and (economic) model predictive control [3.5, 3.6]. Furthermore, RHs energy systems can take advantage of various Strategy Planning Models (SPMs) to decrease the demand and particularly the energy cost at the user demand side. Naidu and Craig [3.7] present a chronological overview of the advanced SPMs implemented on heating, ventilating, and air conditioning (HVAC) and refrigeration systems. Weiss investigates an adaptive neuro energy management SPM in order to decrease the building energy cost [3.8]. Platt and colleagues used demand response experiments in two large office buildings [3.9]. Srinivas and Ning evaluated different demand response programs in order to analyze the benefits of applied SPMs [3.10].

Management and control of RHs energy systems have been extensively researched. For instance, García-Domingo designed a building integrated PV system to analyze the electrical energy balance of the house [3.11]. Keshtkar et al. [3.12] used smart wireless sensors in a residential house in order to reduce the electrical load. Onda et al. [3.13] utilized the storage system of the smart electric vehicle for shifting the house peak load to off-peak hours. Boehm [3.14] examined various approaches, including energy-conserving design and the use of photovoltaic arrays, to reduce the peak electrical demand in residences. Castillo-Cagigal et al. [3.15] examined the use of a semidistributed demand-side management system to improve the house self-consumption capability. Fernandes et al. [3.16] developed a dynamic load management model for enhancing the participation of house in demand response events. Beizaee and colleagues [3.17] used zonal space heating controls to decrease the house demand. Naspolini et al. [3.18] investigated the benefits of solar water heating in house energy demand. Chassin et al. [3.19] examined the cost, comfort and energy impacts of a discrete-time controller in a residential house HVAC system. Li et al. [3.20] developed a dynamic zone modelling in order to reduce the HVAC system electricity cost. Nielsen and Drivsholm [3.21] developed a system in which ventilation was controlled by an intelligent 
demand controller. Among previous research, the ones that concentrate on residential HVAC load [3.17-3.21] are most useful and efficient since HVAC systems consume a significant portion of the total energy used in households. According to the Annual Energy Outlook published by the U.S. Energy Information Administration [3.4], HVAC systems consume more than 40\% of the overall energy in residential houses resulting in higher operating costs and environmental pollution according to the Annual Energy Outlook published by the U.S. Energy Information Administration [3.4]. Over the past decade, numerous strategy planning and energy conservation methods/approaches have been developed to address the planning issues related to managing RHs and their HVAC systems energy demand and associated cost. For example, Ma et al. [3.5] showed that HVAC system energy cost can be reduced using thermal storage in building mass. Candanedo and Athienitis [3.6] examined the effect of floor heating mass on reducing the energy cost. In this study, the impacts of passive solar gains on managing the HVAC system energy demand, were considered. Temperature reset during unoccupied hours [3.7, 3.8], night setback, precooling during off-peak period and set-point change during peak hours [3.9, 3.10], optimum start and stop times [3.22], ventilation control [3.23, 3.24] and economizer cycle control [3.25] are some of the SPMs implemented on HVAC system in order to decrease its energy demand and associated cost.

Many of the previous studies utilized house energy simulators such as TRNSYS [3.26], EnergyPlus [3.27, 3.28], Mathcad [3.29], and esp-r [3.30, 3.31]. These energy simulators offer an excellent opportunity for detailed design and modeling of the house and its HVAC system and provide very accurate results useful for performance analysis and optimization process. In contrast, these simulators do not include sub-models of advanced devices/strategies for controlling the HVAC system operation and suffer from poor control mechanism. As a result, only simple conventional (on/off) controllers were employed in order to control and manage the house and its HVAC system performance. Due to the large thermal inertia of the conditioned zone and dynamic disturbances, the on/off controller cannot accurately regulate the zone temperature resulting in thermal discomfort for the occupants and higher energy costs [3.4, 3.32]. In addition to the lack of advanced controllers, these energy simulators use operational/historical weather dataset (provided in a library file) for simulating the house's energy system. Hence, they inherently offer no mechanism for estimating the future state of their process models based on the forecast weather dataset. In the advanced predictive controller, a model of the system (building and its HVAC system) and the forecast weather conditions are used to determine the best set of control operations 
$[3.4,3.32]$. Hence, no predictive controller can be designed and run within such building energy simulators.

Therefore, in order to control the process models of such software with advanced and/or predictive controllers (i.e., MPC), a software/tool with advanced process control mechanism (i.e. Matlab) should be integrated/linked into these building energy simulators. This chapter discusses the development of the first MPC controller that uses a Matlab-TRNSYS co-simulator for controlling/managing the TRNSYS program. To design the first MPC controller, three novel predictive strategy planning models (SPMs) including Smart Dual Fuel Switching System (SDFSS), Load Shifting (LSH) and LSHSDFSS as the combination of SDFSS and LSH-SPM models are developed in this chapter using Matlab program. In SDFSS-SPM, a smart controller is developed to select the least expensive hot air supplier (between electrical air source heat pump (ASHP) and natural gas mini boiler), in each hour, by taking into consideration the house's thermal demand and Time of Use (TOU) pricing scheme during the decision making process. In LSHSPM, an intelligent mechanism is used to select the best pre-heating/pre-cooling starting time based on the outdoor temperature effects and the dynamic characteristics of the case study house. As the third developed SPM, LSHSDFSS-SPM takes advantage of both novel load shifting and fuel switching systems to offer maximum saving on HVAC system energy cost.

To implement these predictive SPMs on the TRNSYS model, Matlab and TRNSYS programs are linked together to found a Matlab-TRNSYS co-simulator. The comprehensive TRNSYS house and HVAC system process models, which utilized in this chapter, has been previously developed and calibrated based on the characteristics/specifications of a real case study house (Archetype House A) [3.41]. In this chapter, the advantage of the developed MPC controller is examined by investigating the effectiveness of each SPM on HVAC system energy cost saving for the next 24 hours horizon time. In this method of control, the future state of the system is predicted based on the forecast weather dataset, the system model, and control vector signals (generated as the model output) which drive the system towards the desired state. This developed MPC controller, which acts as a smart grid-friendly controller, also has the potential to be utilized as a test bed for implementing various SPMs previously developed for reducing the energy cost of HVAC systems. 


\subsection{Model Description}

\subsubsection{Historical and Forecast Weather Information}

Weather conditions play a significant role in house energy system simulation. As a result, getting access to accurate weather forecast data is vital for simulating the house energy system. Canadian Meteorological Center (CMC) forecasts weather four times a day to ensure the predicted data are highly accurate. In addition, there is a wide range of historical weather dataset on different online sources [3.33] that could be used for testing and verifying the decision making or strategy planning models $[3.34,3.35]$. Figure 3-1 provides an overall view of various available weather data used for different purposes. Deterministic and probabilistic are two separate methods used for generating predicted data. Each method has its own advantages. The deterministic forecast is based on the result of one or two predictive model(s). The data predicted using this method is highly accurate and is usually used for short-term control process [3.34, 3.35].

The probabilistic forecast is the result of a group (sometimes as many as 21) of models producing a range of forecast data. An ensemble of different models generates a dataset with an appropriate range of values. This method is suitable for planning long-term control process [3.34, 3.35].

The actual historical and weather forecast datasets are presented in different formats. Operational weather data is usually presented in spreadsheet (CSV), XML, ASCII (text) and GRIB2 formats $[3.36,3.37]$. However, GRIB2 is the most common format used for presenting the forecast dataset. To be usable in TRNSYS and Matlab, GRIB2 data format should be retrieved into a standard numerical format. To this end, two software have been used in this project. The first one is "wget" [3.38] which is a command-line program designed for retrieving files using HTTP, HTTPS, and FTP protocols. The second one is "NCToolbox" [3.39], a Matlab toolbox designed for working with the datasets generated as the output of GRIB2 retrieving programs [3.34]. 


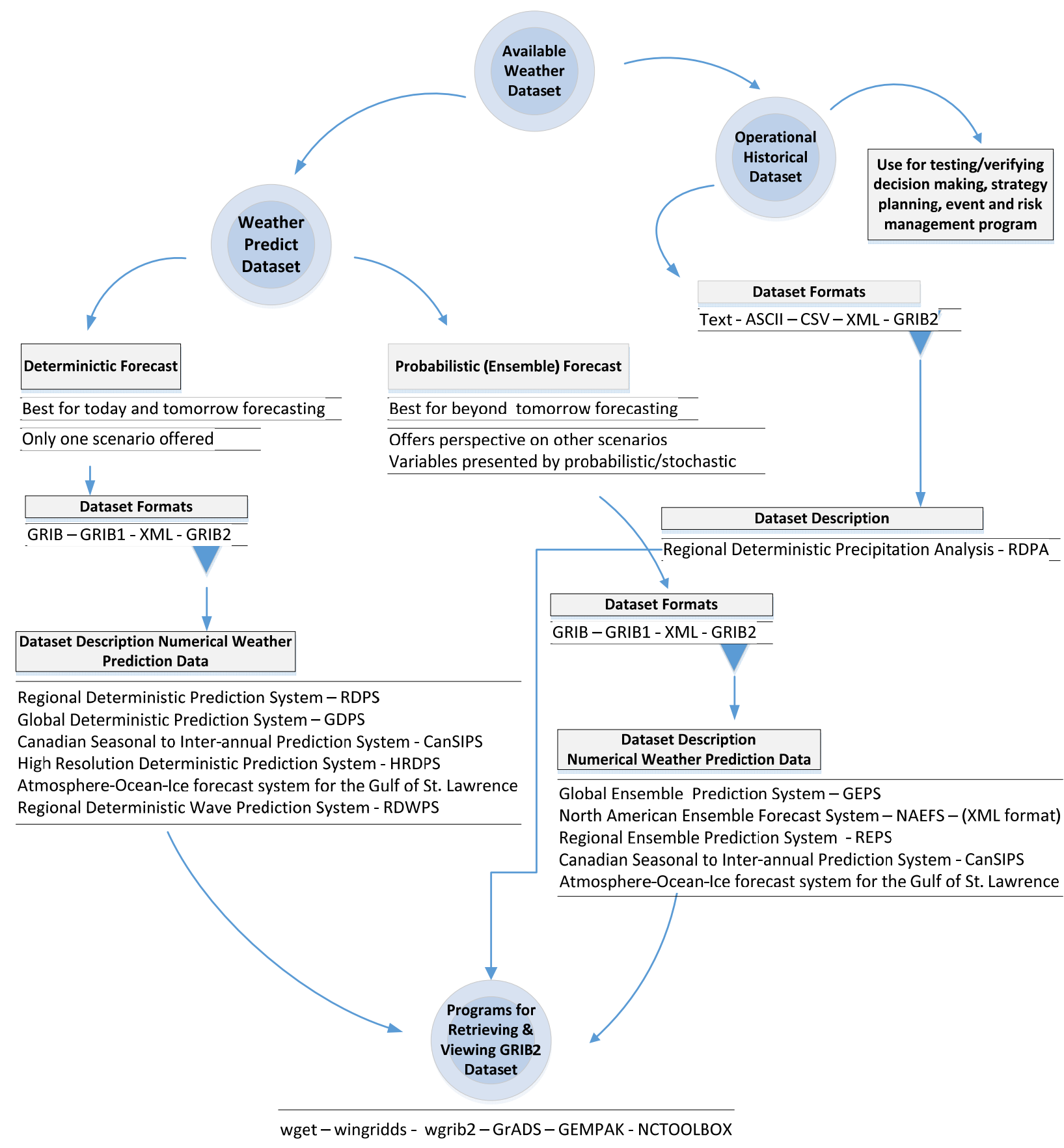

Figure 3-1: The overall view of various available weather datasets

Based on the nature of the project which requires accurate short-term prediction, high resolution deterministic prediction system (HRDPS) with bandage $2.5 \mathrm{~km}$ was selected from CMC website as the forecast dataset. In order to collect this information from the database, wget was installed on a computer and a system command was called from Matlab to collect the necessary data using 
wget $[3.34,3.35]$. This process downloaded 24 hours' worth of weather forecast data onto the system that needed to be further processed and filtered so that only the temperature data for the next 24 hours (as the most important parameter with the greatest impact on the house energy system) is remained. The information that was downloaded off the HRDPS source created a $2.5 \mathrm{~km}$ by $2.5 \mathrm{~km}$ grid across Eastern Ontario and contained the weather information for each element of the grid. Figure 3-2 illustrates the described grid format of the GRIB2 data. In order to process the data, the coordination of the grid element from which the weather forecast is collected must be determined. In this study, the TRNSYS program models thermal energy of House A [3.40, 3.41] located at Kortright Centre for Conservation in Vaughan, Ontario. Therefore, the grid element of $(210,490)$, corresponding to the location of the house, was selected for data collection.

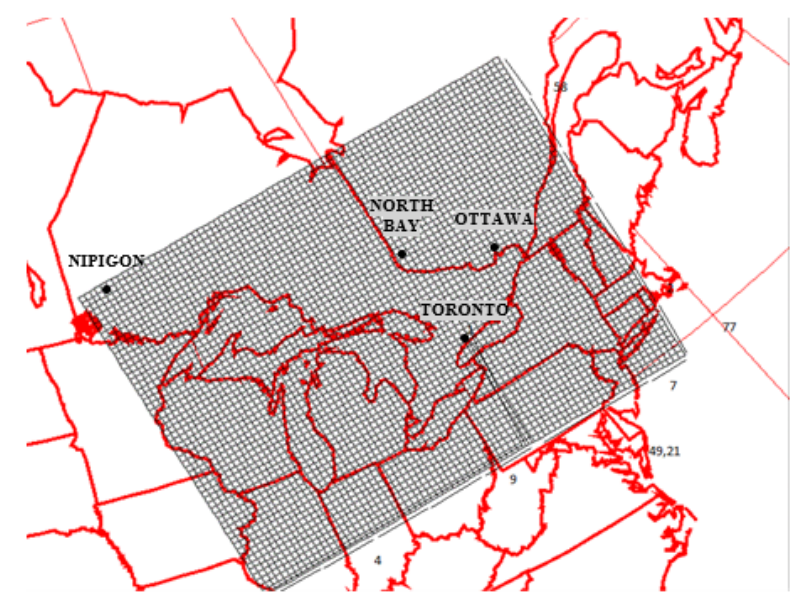

Figure 3-2: The grid format of the GRIB2 data - Eastern Ontario

In order to create an interface between Matlab and the collected GRIB2 data, the NCToolbox was installed, and the ncgeodataset function was used to present all the data stored in each of the 24 downloaded files as a multidimensional matrix. This matrix was filtered to include only the weather forecast information for each hour and then was stored into a single two-dimensional array.

\subsubsection{Estimation of 24 Hours-ahead HVAC System Electrical Demand Based on Weather} Forecast Dataset

\subsubsection{House Description}

The twin Archetype Sustainable Houses (ASH) located at the Kortright Centre for Conservation in Vaughan have been constructed by the Toronto and Region Conservation Authority (TRCA) 
[3.40-3.42]. These twin-houses demonstrate sustainable housing technologies through experimentation and research and are among the first Canadian projects to achieve a Leadership in Energy and Environmental Design (LEED) for Homes Platinum Certification [3.40]. House A uses a two-stage variable capacity air-source heat pump and a natural gas mini boiler for space heating and cooling and domestic hot water (DHW) heating, and was selected for testing different strategy planning models in this project. This house has an air-tight building envelope according to the standards of ASHRAE 90.1 [3.40-3.42].

\subsubsection{TRNSYS Model}

TRNSYS is a transient system energy modeling software designed to solve complex energy system problems [3.41, 3.49]. The House A TRNSYS model, developed by Safa et al. [3.41], is used in this project. According to a study on various building energy modeling programs [3.34, 3.48], TRNSYS is reasonably robust when it comes to HVAC system modeling.

\subsubsection{Methodology}

Different sub-programs with various operational mechanisms are run to achieve the project's objectives. The study starts with downloading and processing of forecast weather data and continues with running the House A TRNSYS model with weather forecast data, implementing strategy planning models on TRNSYS system by generating operational command matrix (control matrix), and lastly post-processing the generated data.

Figure 3-3 illustrates the framework of the house energy simulation system consisting of three different but complementary programs. Wget runs first, downloading short-term weather forecast dataset from the CMC website. The second program is Matlab, which controls the operational process and links other programs in order to transfer the data. The third program is TRNSYS, which is used for simulating the house and its energy systems.

To achieve the project goals, each sub-program should be run at a particular time to generate the inputs for the next program. To control and manage this sequence, a master director program is required to drive each process on time. In this project, Matlab plays this role by handling the process, linking different programs as well as storing the data or calling the required data. 


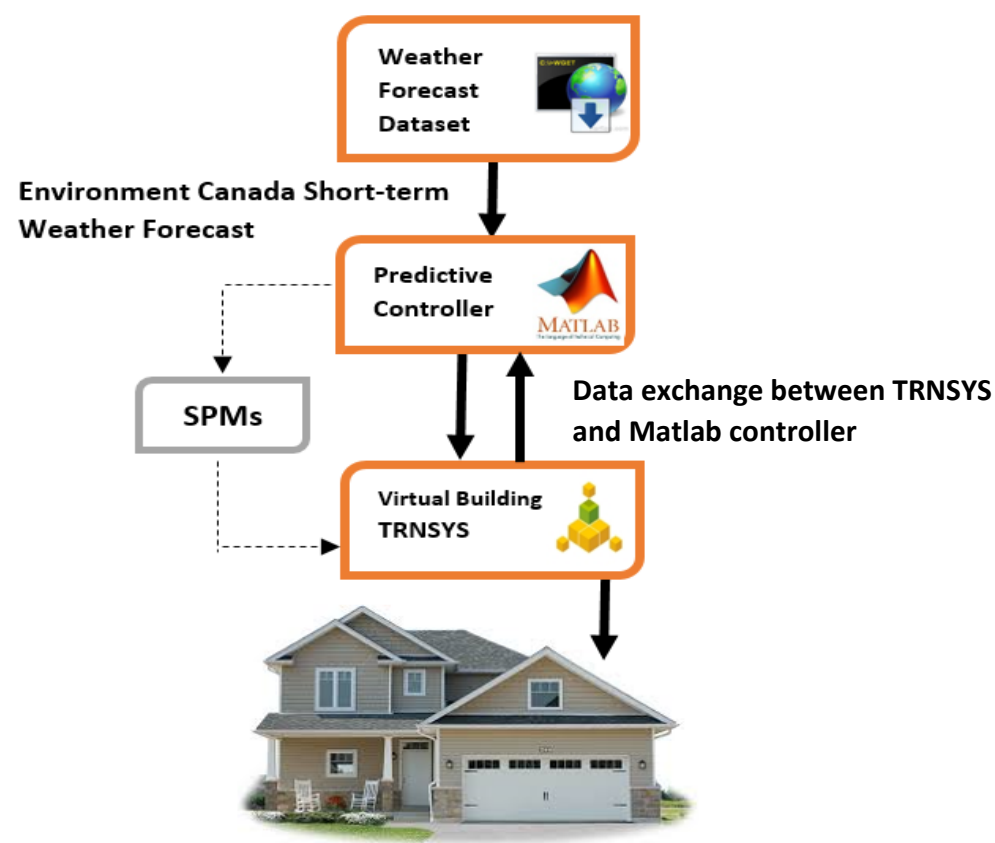

Figure 3-3: Framework of the house energy simulation system

Figure 3-4 depicts the overall process in a simple flowchart. As Figure 3-4 shows, all retrieved weather forecast data are initially recorded with Matlab on an Excel lookup table. The lookup table is the only method used for importing external data into the TRNSYS program. In the next step, TRNSYS program is called by Matlab to read the weather forecast data from Excel file lookup table and perform House A energy simulation for the next 24 hours. Then, all generated data including the hourly thermal demand of House A and air source heat pump (ASHP) electric demand are registered into another Excel file which will be used for post processing.

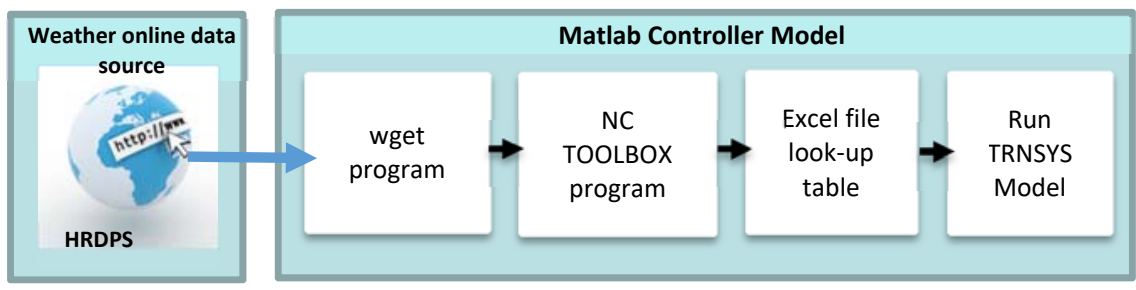

Figure 3-4: Framework of Matlab Controller Program

The operational command matrix that generated by Matlab predictive controller based on each ECSPM is written to the Excel file in a lookup table. This lookup table is then read by TRNSYS 
in order to control the ASHP operation. This control mechanism, which acts as a thermostat module in TRNSYS program, is used to take care of lower and upper comfort level temperatures. Based on the ASHRAE Standard [3.45], the indoor temperature during the occupied period of the heating season should be kept between $20^{\circ} \mathrm{C}$ and $24^{\circ} \mathrm{C}$ for thermal comfort. In this project, to ensure the ASHRAE Standard, when the zone (1st floor) temperature is lower than the minimum permitted temperature $\left(20^{\circ} \mathrm{C}\right)$, a trigger command (" 1 " signal) is sent to the ASHP to turn it on. When the zone temperature is higher than the maximum permitted temperature $\left(24^{\circ} \mathrm{C}\right)$, command " 0 " is sent to the ASHP to turn it off. This command mechanism is used for implementing different strategy planning models presented in the next section.

\subsubsection{Process Time Step}

Initially, all simulations were performed based on one-hour time step. However, the results were not very accurate since the events taking place during a given hour could not be monitored/processed by the control algorithm. After a few trials, it is found that this problem can be avoided using 5-minute time step. Although with 5-minute time step it would take longer to simulate the model, the operation of the HVAC system would be controlled and monitored more accurately and the data would be measured more precisely. With 5-minute time step, TRNSYS model is run twelve times per hour (289 times per day) to generate the result. Figure 3-5 shows the operational command matrix generated after implementing weather forecast data illustrated in Figure 3-6. Since 5-minute is selected as the time step, each hour contains 12 time steps/cells. For example, hour 0 consists of 12 cells and each cell determines the on/off condition of HVAC system

\begin{tabular}{|c|c|c|c|c|c|c|}
\hline First 6 Hours & 0 & 1 & 2 & 3 & 4 & 5 \\
\hline Command Signals & 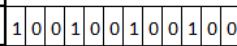 & $\begin{array}{lllllllllllllll}1 & 1 & 1 & 1 & 1 & 0 & 1 & 0 & 0 & 1 & 0 & 0 \\
\end{array}$ & $\begin{array}{lllllllllllllll}1 & 1 & 0 & 1 & 0 & 1 & 0 & 1 & 0 & 1 & 0 & 1 \\
\end{array}$ & $\begin{array}{lllllllllllllll}0 & 1 & 0 & 1 & 0 & 1 & 0 & 1 & 0 & 1 & 0 & 1\end{array}$ & $\begin{array}{llllllllllllllll}0 & 1 & 0 & 1 & 0 & 1 & 0 & 1 & 0 & 1 & 0 & 1 \\
\end{array}$ & \begin{tabular}{l|llllllllllllll}
0 & 1 & 0 & 1 & 0 & 1 & 0 & 1 & 0 & 1 & 0 & 1 \\
\end{tabular} \\
\hline Second 6 Hours & 6 & 7 & 8 & 9 & 10 & 11 \\
\hline Command Signals & $\begin{array}{lllllllllllllll}0 & 1 & 0 & 1 & 0 & 1 & 0 & 1 & 0 & 1 & 0 & 1 \\
\end{array}$ & $\begin{array}{lllllllllllllll}0 & 1 & 0 & 1 & 0 & 1 & 0 & 1 & 0 & 1 & 0 & 1 \\
\end{array}$ & $\begin{array}{lllllllllllllll}0 & 0 & 1 & 0 & 1 & 0 & 1 & 0 & 1 & 0 & 1 & 0 \\
\end{array}$ & $\begin{array}{llllllllllllll}1 & 0 & 1 & 0 & 1 & 0 & 1 & 0 & 1 & 0 & 1 & 0 \\
\end{array}$ & $\begin{array}{llllllllllllllll}1 & 0 & 1 & 0 & 1 & 0 & 1 & 0 & 1 & 0 & 1 & 0 \\
\end{array}$ & $\begin{array}{lllllllllllllll}1 & 0 & 1 & 0 & 1 & 0 & 1 & 0 & 1 & 0 & 1 & 0 \\
\end{array}$ \\
\hline Third 6 Hours & 12 & 13 & 14 & 15 & 16 & 17 \\
\hline Command Signals & 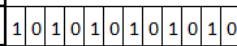 & \begin{tabular}{lllllllll|l|l|lll}
1 & 0 & 1 & 0 & 1 & 0 & 1 & 0 & 1 & 0 & 1 & 0 \\
\end{tabular} & \begin{tabular}{lllllllll|l|l|l|l}
1 & 0 & 1 & 0 & 1 & 0 & 1 & 0 & 1 & 0 & 1 & 0 \\
\end{tabular} & \begin{tabular}{lllllllll|l|l|l|l}
1 & 0 & 1 & 0 & 1 & 0 & 1 & 0 & 1 & 0 & 1 & 0 \\
\end{tabular} & 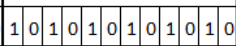 & $\begin{array}{lllllllllllllll}1 & 0 & 1 & 0 & 1 & 0 & 1 & 0 & 1 & 0 & 1 & 0 \\
\end{array}$ \\
\hline Fourth 6 Hours & 18 & 19 & 20 & 21 & 22 & 23 \\
\hline Command Signals & 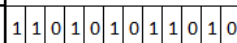 & \begin{tabular}{lllllllll|l|l|l}
1 & 0 & 1 & 1 & 0 & 1 & 0 & 1 & 0 & 1 & 0 & 1
\end{tabular} & \begin{tabular}{lllllll|l|l|l|l|l}
1 & 0 & 1 & 0 & 1 & 0 & 1 & 1 & 0 & 1 & 0 & 1 \\
\end{tabular} & \begin{tabular}{lllllllllllll|l}
0 & 0 & 0 & 0 & 1 & 0 & 1 & 0 & 1 & 1 & 0 & 1 \\
\end{tabular} & \begin{tabular}{lllllllll|l|l|l|l}
0 & 0 & 0 & 1 & 0 & 1 & 0 & 1 & 0 & 1 & 0 & 1 \\
\end{tabular} & \begin{tabular}{lllllllll|l|l|l}
0 & 0 & 0 & 0 & 1 & 0 & 1 & 0 & 1 & 0 & 1 & 0 \\
\end{tabular} \\
\hline
\end{tabular}

Figure 3-5: Operational command matrix generated with Matlab program after using weather forecast data

in that particular time step. Figure 3-7 illustrates the same operational command matrix as a graph generated with TRNSYS program. 
In this project, $22^{\circ} \mathrm{C}$ is considered as the initial zone temperature. Figure $3-8$ shows the zone temperature curve during the 24 hour simulation time. As the figure shows, the zone temperature started at $22^{\circ} \mathrm{C}$ and fluctuated around $20.7^{\circ} \mathrm{C}$, the temperature selected as the heating set point temperature during the 24 hours by the TRNSYS model developers. This set point has been set in the lookup table as the heating set point temperature before implementing any SPM. The essential goal of this project is to design a grid-friendly house by using different strategy planning models in order to shift the load to off-peak hours and minimize the HVAC system's daily energy cost. In this section, the electricity consumed by ASHP is considered the only fuel consumed by the HVAC system.

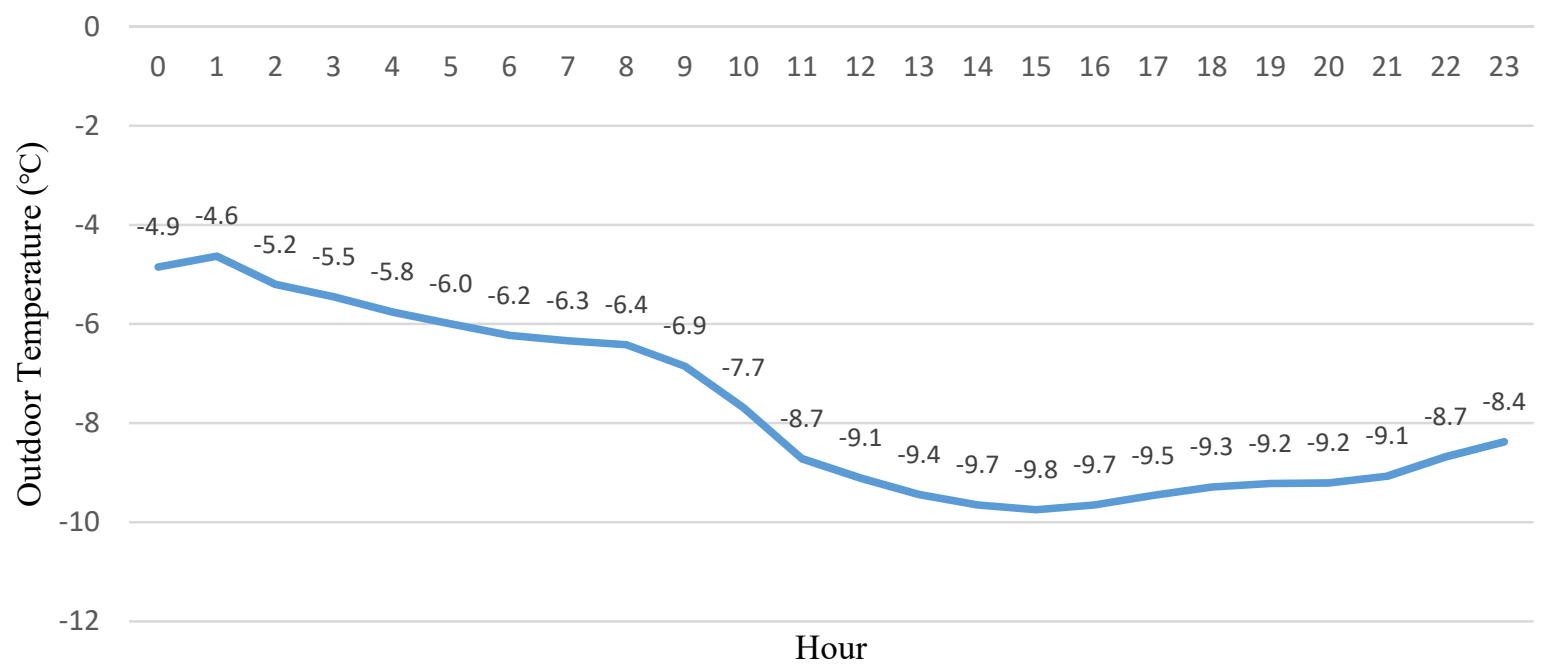

Figure 3-6: Outdoor temperature on Jan $4^{\text {th }}, 2015$ based on forecast weather dataset

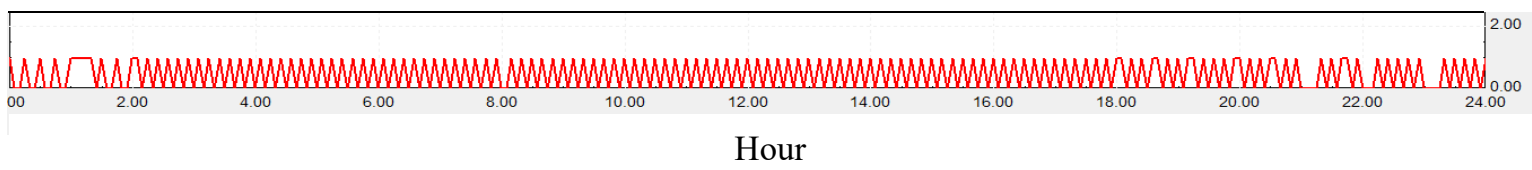

Figure 3-7: Operational command matrix graph - TRNSYS program 


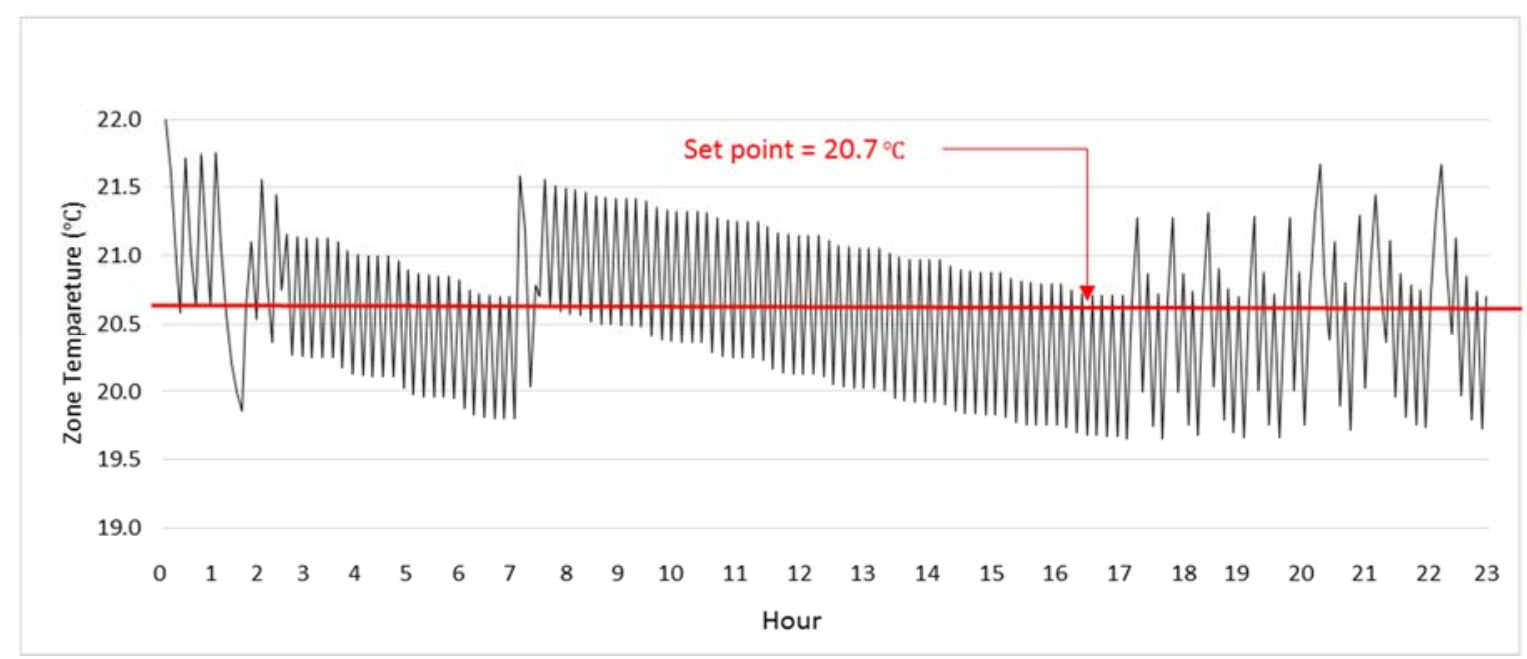

Figure 3-8: Zone (1st floor) Temperature after running TRNSYS program with weather forecast data

\subsubsection{Electricity Prices}

Distribution System Operators (DSOs) apply rates that penalize energy use during peak hours via demand charges and/or time of use (TOU) rates. Figure 3-9 shows the price of electricity in Ontario since November $1^{\text {st }}, 2014$ for winter and summer. The electricity prices used in this project are $11.70 \phi / \mathrm{kWh}, 15.40 \phi / \mathrm{kWh}$ and $18.00 \phi / \mathrm{kWh}$ for off-peak, mid-peak and peak hours, respectively. These prices are estimated using the Ontario Energy Board (OEB) TOU electricity prices [3.50].

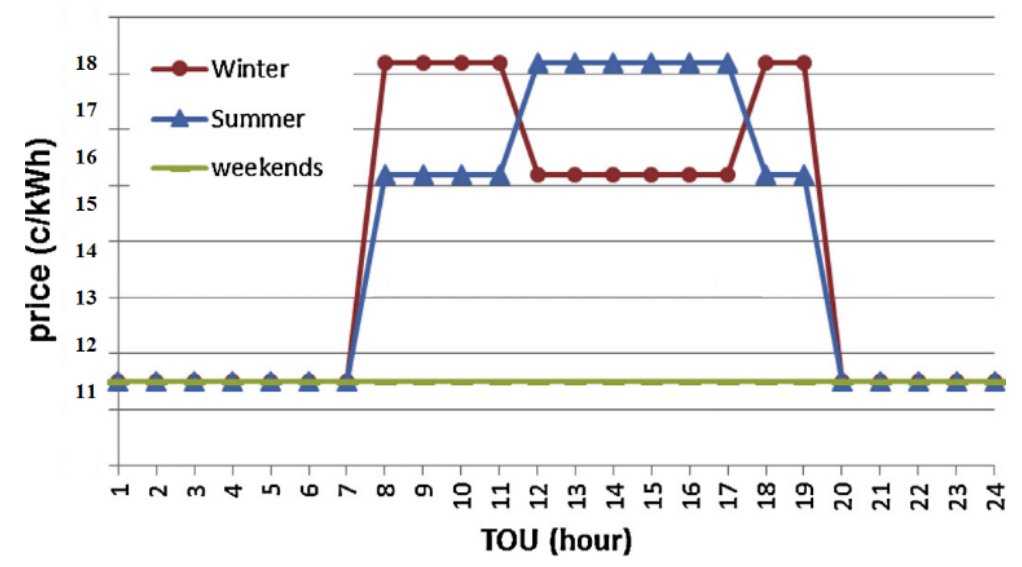

Figure 3-9: Price of electricity in Ontario since November $1^{\text {st }}, 2014$ for winter and summer [3.50] 
3.2.3 HVAC System Energy Cost Reduction with Smart Dual Fuel Switching System Strategy Planning Model (SDFSS-SPM)

The test house, House A, has a natural gas mini boiler and an electric two-stage variable capacity ASHP to generate hot air through the air handling unit (AHU) to meet the space heating demand. The objective of this strategy planning model is to reduce the HVAC system energy cost by selecting the least expensive hot air supplier at each particular hour. After selecting the least expensive hot air supplier, the corresponding system (ASHP or mini boiler) would be set up to meet the space heating demand.

\subsubsection{Estimating ASHP Fuel Cost}

Outdoor temperature and electricity price are the two most important parameters affecting the ASHP energy cost. Outdoor temperature directly affects the ASHP coefficient of performance (COP). Figure 3-10 shows the experimentally validated House A ASHP COP curve [3.41, 3.50]. After determining COP and electricity price for a given hour, the cost of the unit of energy produced by the ASHP is calculated using Equation (3-1):

$$
\text { ASHP electricity cost for preparing one unit of heat energy }(\$ / k W h)=\frac{\text { Electricity price }(\ell / k W h)}{\operatorname{COP*100}}
$$

where Electricity price indicates the cost of electricity $(\$ / k W h)$ at the given hour based on TOU pricing scheme and COP shows the ASHP coefficient of performance determined based on the outdoor temperature at the given hour.

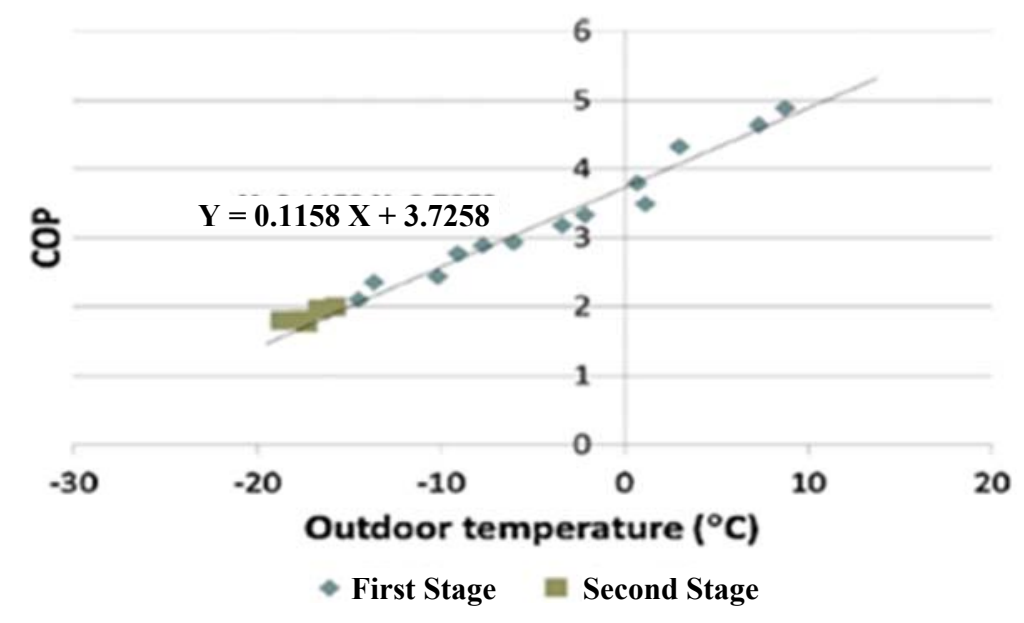

Figure 3-10: House A ASHP COP validated with outdoor temperature [3.50] 


\subsubsection{Estimating Mini Boiler Fuel Cost}

Natural gas price (41.60 $\varnothing / \mathrm{m}^{3}$, determined based on OEB prices) is the same for all hours. Thus, mini boiler efficiency is the only variable used to determine the cost of each unit of thermal energy produced by the boiler. The efficiency of the boiler is determined based on the flow rate of water (load percentage) circulating through the boiler. Figure 3-11 illustrates the mini boiler efficiency curve provided by the manufacturer [3.44]. The fuel cost of mini boiler (for preparing one unit of thermal energy) is calculated by Equation (3-2):

Mini boiler natural gas cost for preparing one unit of heat energy $(\$ / k W h)=\frac{\text { Natural Gas price }\left(\$ / m^{3}\right)}{\text { Efficiency } * 10.3}$

where Natural Gas price indicates the cost of natural gas $\left(\$ / \mathrm{m}^{3}\right)$ and Efficincy shows the mini boiler efficiency determined based on the load percentage at the given time. The 10.3 constant is used for converting $1 \mathrm{~m}^{3}$ of natural gas energy content (heating value) into $\mathrm{kWh}$.

At each particular hour, the cheaper hot air supplier is directly selected by comparing the expected fuel cost of ASHP and mini boiler. At House A, the ASHP is selected as the primary hot air supplier and the mini boiler is used just as a backup system. This prioritization can be changed by sending a command signal to AHU controller relay.

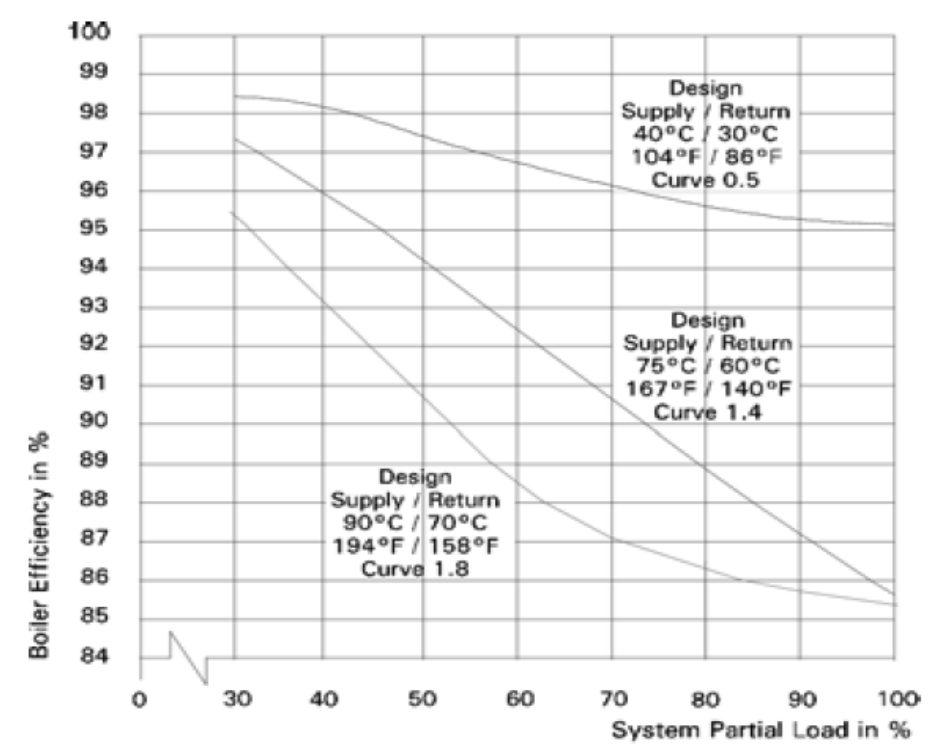

Figure 3-11: VIESMAN Co Technical Data Manual - Mini boiler efficiency curve [3.44] 


\subsubsection{Using SDFS System as a Strategy Planning Model}

Figure 3-12 shows the operational boundaries of ASHP and mini boiler classified according to off, mid- and peak-hours. These functional limits/switching points are calculated by solving Equations (3-1) and (3-2) at different TOU prices and outdoor temperatures. In Figure 3-12, the

minimum and maximum temperatures are selected based on the minimum and maximum temperatures of Toronto reported by Environment Canada [3.45, 3.46].

As the figure shows, $-14^{\circ} \mathrm{C}$ is the switching point during off-peak hours. In other words, during the off-peak hours when the outdoor temperature falls below $-14^{\circ} \mathrm{C}$, a unit of heat energy generated by the mini boiler becomes less expensive than a unit of heat energy generated by the ASHP. Based on this methodology, the switching points at mid-peak and on-peak hours are $-5^{\circ} \mathrm{C}$ and $0^{\circ} \mathrm{C}$, respectively.
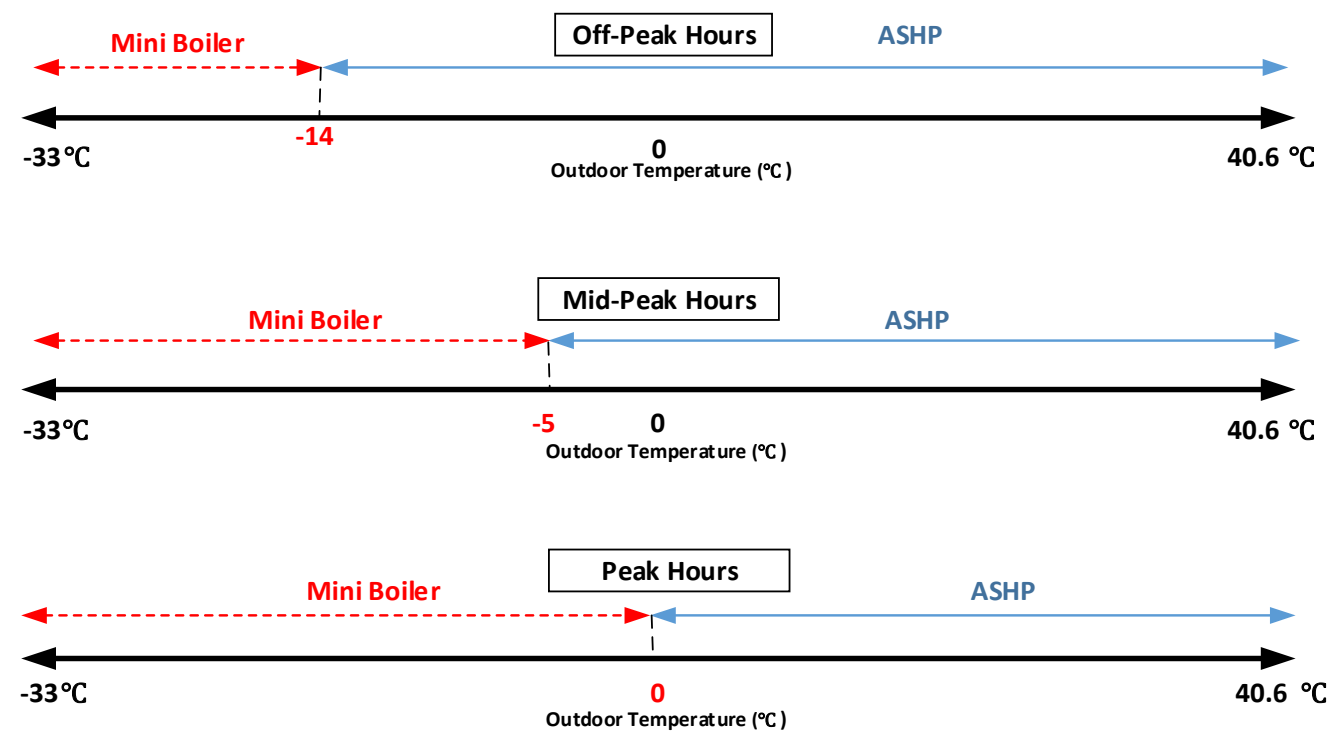

Figure 3-12: Switching points on different TOU prices

Figure 3-13 demonstrates the SDFSS strategy planning model used for determining the switching point at different hours. The HVAC daily fuel cost could be minimized by modifying the operational command matrix presented in Section 3.2.2 based on the results of the SDFSS strategy planning model. 


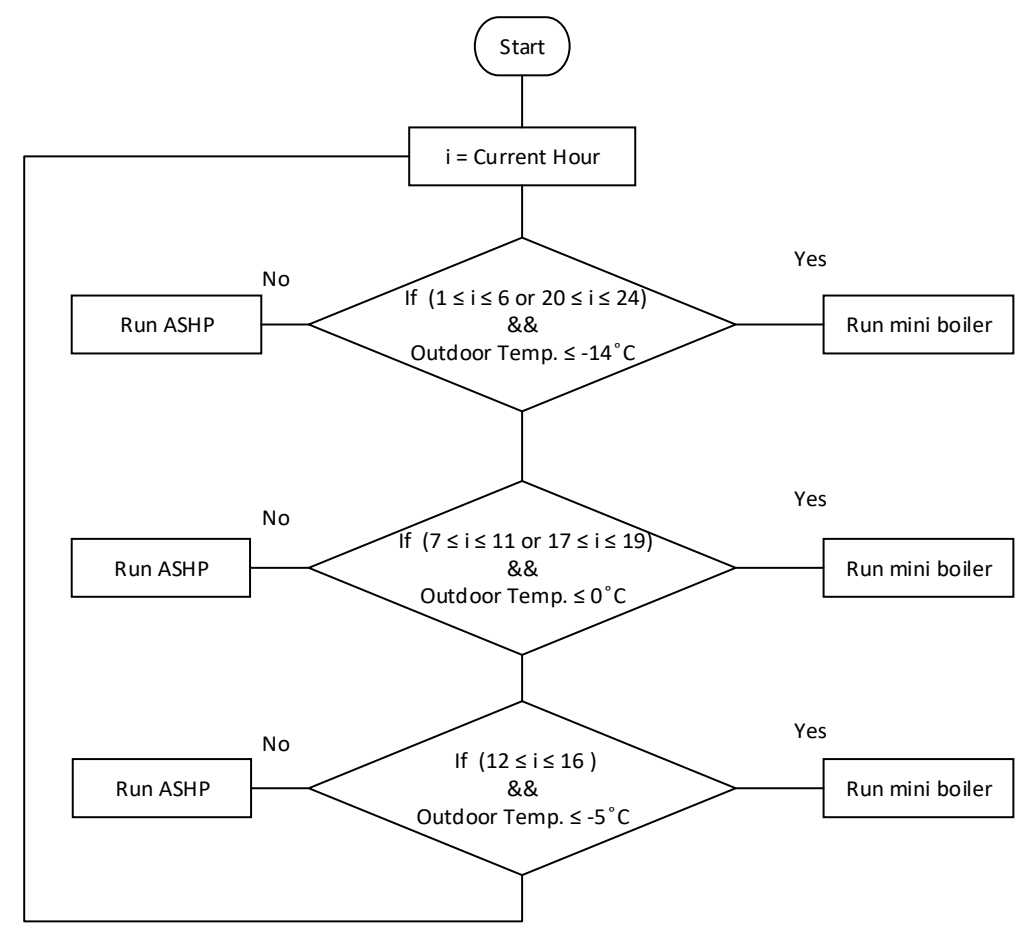

Figure 3-13: SDFSS strategy planning model

Figure 3-14 shows the developed operational command matrix after using SDFSS-SPM. In this Figure ' $\mathrm{A}$ ' and ' $\mathrm{M}$ ' represent the ASHP and mini boiler, respectively, and ' $\mathrm{O}$ ' marks the time at which the HVAC system was off. Based on Figure 3-6, since the outdoor temperature during the peak and mid-peak hours is below the $0^{\circ} \mathrm{C}$ and $-5^{\circ} \mathrm{C}$, the mini boiler is selected as the cheaper hotair supplier system during these hours. In practical application, an appropriate time lag should be set up in SDFSS controller to eliminate frequently switching between mini boiler and ASHP at contiguous time steps.

\begin{tabular}{|c|c|c|c|c|c|c|}
\hline First 6 Hours & 0 & 1 & 2 & 3 & 4 & 5 \\
\hline Command Signals & A 0 O & 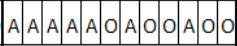 & 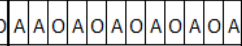 & 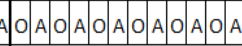 & 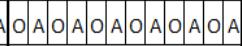 & 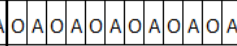 \\
\hline Second 6 Hours & 6 & 7 & 8 & 9 & 10 & 11 \\
\hline Command Signals & O A O A $\mathrm{O}$ A & $\mathrm{OMOMOMOMOMOM}$ & lo $\mathrm{momomomo} \mathrm{mo}$ & $\mathrm{Omomomo} \mathrm{mo} \mathrm{mo} \mathrm{mo}$ & $\mathrm{mom} \mathrm{Mm} \mathrm{M} \mathrm{O} \mathrm{Momo}$ & $\mathrm{momomomo} \mathrm{Mo} \mathrm{mo}$ \\
\hline Third 6 Hours & 12 & 13 & 14 & 15 & 16 & 17 \\
\hline Command Signals & 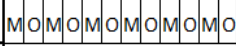 & 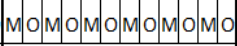 & $\mathrm{mom} \mathrm{M} \mathrm{Momo} \mathrm{mo} \mathrm{mo}$ & $\mathrm{O} \mathrm{mo} \mathrm{mo} \mathrm{mo} \mathrm{mo} \mathrm{Mo} \mathrm{mo}$ & $\mathrm{mol} \mathrm{M} \mathrm{MOM} \mathrm{OMO} \mathrm{MO}$ & $\mathrm{M} \mathrm{O} \mathrm{MO} \mathrm{MO} \mathrm{MO} \mathrm{MO} \mathrm{MO}$ \\
\hline Fourth 6 Hours & 18 & 19 & 20 & 21 & 22 & 23 \\
\hline Command Signals & $\mathrm{MmOMO} \mathrm{MO} \mathrm{Mm} \mathrm{M} \mathrm{Mo}$ & A $O$ A & 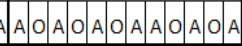 & A 0 O 0 O & 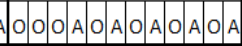 & 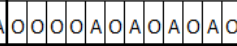 \\
\hline
\end{tabular}

Figure 3-14: Developed operational command matrix after using SDFSS-SPM 


\subsubsection{Calculating the Impact of SDFSS-SPM on HVAC System Daily Fuel Cost (DFC) at Different Outdoor Temperatures}

To calculate the impact of SDFSS-SPM on DFC, the Matlab model was run when the daily average outdoor temperature was fluctuating between $-20^{\circ} \mathrm{C}$ and $0^{\circ} \mathrm{C}$. Table $3-1$ demonstrates the simulation results.

Table 3-1: Comparison between DFC before and after running SDFSS-SPM

\begin{tabular}{cccccc}
\hline $\begin{array}{c}\text { Daily Average } \\
\text { Outdoor } \\
\text { Temperature } \\
\left({ }^{\circ} \mathrm{C}\right)\end{array}$ & $\begin{array}{c}\text { Maximum } \\
\text { Outdoor } \\
\text { Temperature } \\
\left({ }^{\circ} \mathrm{C}\right)\end{array}$ & $\begin{array}{c}\text { Minimum } \\
\text { Outdoor } \\
\text { Temperature } \\
\left({ }^{\circ} \mathrm{C}\right)\end{array}$ & $\begin{array}{c}\text { DFC before } \\
\text { Running } \\
\text { SDFSS-SPM } \\
(\boldsymbol{\Phi})\end{array}$ & $\begin{array}{c}\text { DFC after } \\
\text { Running } \\
\text { SDFSS-SPM } \\
(\Phi)\end{array}$ & $\begin{array}{c}\text { Energy } \\
\text { Cost } \\
\text { Saving } \\
(\Phi)\end{array}$ \\
\hline $\mathbf{0}$ & 3 & -2 & 265.64 & 265.64 & 0.00 \\
$\mathbf{- 2}$ & 3 & -4 & 287.74 & 276.20 & 11.54 \\
$\mathbf{- 4}$ & 2 & -5 & 308.85 & 290.85 & 18.00 \\
$\mathbf{- 6}$ & 0 & -8 & 343.78 & 317.14 & 26.64 \\
$\mathbf{- 8}$ & -2 & -10 & 366.51 & 336.24 & 30.27 \\
$\mathbf{- 1 0}$ & -6 & -13 & 389.24 & 345.34 & 43.90 \\
$\mathbf{- 1 2}$ & -6 & -16 & 515.93 & 452.19 & 63.74 \\
$\mathbf{- 1 4}$ & -8 & -16 & 705.30 & 543.15 & 162.16 \\
$\mathbf{- 1 6}$ & -12 & -18 & 870.80 & 562.91 & 307.89 \\
$\mathbf{- 1 8}$ & -14 & -21 & 1030.27 & 612.63 & 417.64 \\
$\mathbf{- 2 0}$ & -15 & -23 & 1194.80 & 666.33 & 528.47 \\
\hline
\end{tabular}

As Table 3-1 shows, the energy cost saving rate continuously increased as the outdoor temperature decreased. This is because the ASHP COP, the parameter with the greatest effect on potential energy cost saving, varies based on the outdoor temperature.

According to Figure 3-10, ASHP COP changes between 1.64 and 5.31. One of the most significant trends in ASHP COP is the rate of reduction of COP, which directly affects the rate of energy cost saving. As outdoor temperature decreases, the rate of reduction of COP increases.

Looking at Table 3-1, it is concluded that the role of SDFSS-SPM on DFC and subsequently fuel cost saving is significantly greater during cold and very cold weather conditions. For example at $20^{\circ} \mathrm{C}, \$ 5.28$ could be saved by using SDFSS-SPM for a day. Figure 3-15 shows the savings due to using SDFSS-SPM in different outdoor temperatures. As the Figure 3-15 shows, SDFSS-SPM is more efficient in energy cost saving when the outdoor temperature is colder. 
Impact of SDFSS System on HVAC system Fuel Cost at

Case Study House

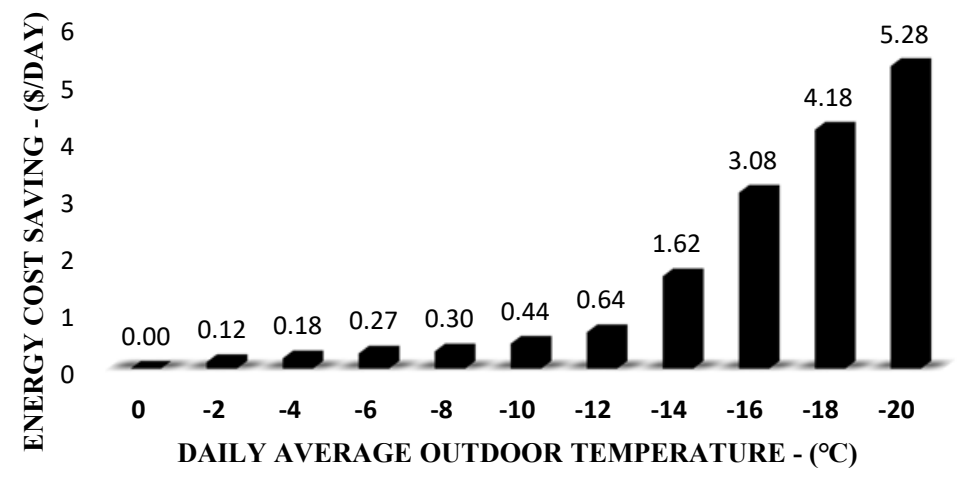

Figure 3-15: Energy cost saved by using SDFSS-SPM at House A at different outdoor temperatures

\subsubsection{HVAC System Demand Management Using Load Shifting Strategy Planning Model (LSH-SPM)}

Buildings are complex entities. Many variables such as building construction/material, equipment capacity, solar irradiance, thermal mass, occupant behavior, and outdoor condition significantly affect the result of strategy planning and especially load shifting models in buildings. Since buildings are part of the total energy system, their behavior has a significant influence on the entire energy network. For example, in terms of peak loads, managing the load of a building's HVAC system by shifting the load from peak to off-peak hours can notably decrease the grid overloading during peak hours. This is achieved by designing smart strategy planning models to avoid electricity consumption during peak hours. The only parameter that significantly affects the thermal energy stored in a house is outdoor temperature. When it is not very cold outside, it is possible to store the heat energy inside the house for immediate future use. However, in cold and extremely cold weather, this is less likely. It should also be noted that house construction/component, interior design, wall layers/thickness, thermal mass, house orientation, and windows size have significant impacts on the capacity of stored thermal energy and consequently the behavior of the house in cold and very cold outdoor temperature. The R-value of an insulating material is a measure of its thermal resistance. The higher the R-value, the more effective the insulator. In the case study house, the R-values of basement walls, wall's insulation, and roof are R-20, R-30, and R-40, respectively. A light thermal mass material has been considered 
for the house in the TRNSYS model. House A was assumed to have four occupants (two adults and two children) with sensible internal heat gain of $2.4 \mathrm{kWh} /$ day.

\subsubsection{Methodology}

As mentioned in Section 3.2.2.4, TRNSYS simulation time step was 5 minutes. However, considering the house heating space, the thermal energy stored in House A after ASHP has been running for 5 minutes is not sufficient to keep the ASHP off in the next five minutes. On the other hand, the zone temperature drops quickly soon after ASHP is turned off. In this case, to improve the efficiency of LSH-SPM, 15 minutes is selected as a time step for controlling the operation of ASHP system during peak hours. Since LSH-SPM is used during peak hours, simulation results showed that keeping ASHP on for more than 15 minutes increased the ASHP consumption, which is not ideal according to load shifting strategy.

\subsubsection{Pre-heating Starting Time (Winter Operation)}

The HVAC load cannot be shifted to off/mid-peak hours without storing thermal energy with the pre-heating process. The most important issue before starting a pre-heating process is determining the pre-heating starting time. The starting time changes based on different parameters. The first and most important of these parameters is the outdoor temperature during the hour prior to the peak hours (i.e., 6:00 am and 16:00 pm in winter). The second parameter is a set of house characteristics, and the third one is zone temperature before starting the pre-heating process.

If the pre-heating process starts sooner than its optimum time, more electricity will be consumed and the room temperature will exceed the ASHRAE Standard range. If the pre-heating starts later than its optimum time, the stored heat energy will not be sufficient to support fully LSH-SPM during peak load hours. In this study, House A TRNSYS model was run at each outdoor temperature to seek the optimum starting time. To this end, first the HVAC set-point was set to $20.7^{\circ} \mathrm{C}$ (the heating set-point temperature selected by TRNSYS model developers). Then, the HVAC system was turned on after 6:00 am (rise up time) and was forced to remain on till zone temperature reached its maximum permitted temperature $\left(24^{\circ} \mathrm{C}\right)$, also known as the saturation point. The time constant (TC) factor, which is used to calculate the starting time, was obtained by subtracting rise up and saturation point times. The time constant factor ensures that the maximum 
heating energy is stored in the house without the zone temperature exceeding the upper comfort level $\left(24^{\circ} \mathrm{C}\right)$. Table 3-2 summarizes the simulation results.

Table 3-2: TRNSYS simulation results - Calculating TC factor

\begin{tabular}{ccccccc}
\hline $\begin{array}{c}\text { Outdoor } \\
\text { Temperature (OT) } \\
\left({ }^{\circ} \mathrm{C}\right)\end{array}$ & $\begin{array}{c}\text { Rise up } \\
\text { Time } \\
(\mathbf{a m})\end{array}$ & $\begin{array}{c}\text { Saturation } \\
\text { Time } \\
(\mathbf{a m})\end{array}$ & $\begin{array}{c}\text { Duration } \\
\text { (TRNSYS } \\
\text { - Scale) }\end{array}$ & $\begin{array}{c}\text { TC } \\
\text { Factor } \\
\text { (minutes) }\end{array}$ & $\begin{array}{c}\text { Pre- } \\
\text { heating } \\
\text { Starting } \\
\text { time (am) }\end{array}$ & $\begin{array}{c}\text { Pre- } \\
\text { heating } \\
\text { Starting } \\
\text { time (pm) }\end{array}$ \\
\hline $\mathbf{2 0}<\mathbf{O T} \leq \mathbf{1 7}$ & 6.41 & 6.58 & 0.17 & 10 & 6.50 & 16.50 \\
$\mathbf{1 7}<\mathbf{O T} \leq \mathbf{1 4}$ & 6.41 & 6.66 & 0.25 & 15 & 6.45 & 16.45 \\
$\mathbf{1 4}<\mathbf{O T} \leq \mathbf{1 1}$ & 6.33 & 6.58 & 0.25 & 15 & 6.45 & 16.45 \\
$\mathbf{1 1}<\mathbf{O T} \leq \mathbf{8}$ & 6.41 & 6.69 & 0.28 & 17 & 6.43 & 16.43 \\
$\mathbf{8}<\mathbf{O T} \leq \mathbf{5}$ & 6.41 & 6.75 & 0.34 & 20 & 6.40 & 16.40 \\
$\mathbf{5}<\mathbf{O T} \leq \mathbf{2}$ & 6.41 & 6.75 & 0.34 & 20 & 6.40 & 16.40 \\
$\mathbf{2}<\mathbf{O T} \leq-1$ & 6.33 & 6.83 & 0.50 & 30 & 6.30 & 16.30 \\
$\mathbf{- 1}<\mathbf{O T} \leq-4$ & 6.41 & 7.08 & 0.67 & 40 & 6.20 & 16.20 \\
$\mathbf{- 4}<\mathbf{O T} \leq-\mathbf{7}$ & 6.41 & 7.11 & 0.70 & 42 & 6.18 & 16.18 \\
$\mathbf{- 7}<\mathbf{O T} \leq-\mathbf{1 0}$ & 6.50 & 7.15 & 0.65 & 39 & 6.21 & 16.21 \\
$\mathbf{- 1 0}<\mathbf{O T} \leq-13$ & 6.41 & 6.75 & 0.34 & 20 & 6.40 & 16.40 \\
\hline $\mathbf{- 1 3}<\mathbf{O T} \leq-16$ & 6.41 & 6.58 & 0.17 & 10 & 6.50 & 16.50 \\
\hline $\mathbf{- 1 6}<\mathbf{O T} \leq-19$ & 6.41 & 6.58 & 0.17 & 10 & 6.50 & 16.50 \\
\hline $\mathbf{- 1 9}<\mathbf{O T} \leq-22$ & 6.41 & 6.58 & 0.17 & 10 & 6.50 & 16.50 \\
\hline $\mathbf{- 2 2}<\mathbf{O T} \leq-25$ & 6.41 & 6.50 & 0.09 & 05 & 6.55 & 16.91 \\
\hline
\end{tabular}

For outdoor temperatures between $17^{\circ} \mathrm{C}$ and $20^{\circ} \mathrm{C}$ (warm outdoor temperatures), the TC is 10 minutes. This means pre-heating should start 10 minutes before the peak hours begin (i.e., 6:50 am or 16:50 pm).

From the simulation results, it is concluded that when it is warm (outdoor temperature of $17^{\circ} \mathrm{C}$ or higher) a shorter time is required to pre-heat the house. This is because heat loss is low due to the high outdoor temperature. TC increases by dropping the outdoor temperature. For example, TC increases to a maximum of 42 minutes when the outdoor temperature is between $-7^{\circ} \mathrm{C}$ and $-4^{\circ} \mathrm{C}$. However, during cold outdoor temperatures, TC factor decreases, reaching 5 minutes at $-25^{\circ} \mathrm{C}$. This is because at the very cold temperatures, the HVAC system will be working almost continuously and there is no time left for the pre-heating process.

In winter, peak hours start at 7:00 am and end at 11:00 am. In order to design a grid-friendly house, HVAC system should remain off during this period. Since this is the best/optimum scenario from the local grid point of view, in this chapter, such situation is called the best case scenario (BCS). However, BCS cannot be utilized all time. The only parameter that poses a problem for using this SPM is the outdoor temperature. To examine the effect of outdoor temperature on zone 
temperature and to determine whether the zone temperature remained within the ASHRAE Standard range in each hour, TRNSYS model was run based on weather forecast data. If zone temperature falls below the standard range in any time step, the HVAC system will turn on during that specific time step. With this strategy planning model, the most appropriate operational command matrix with respects to ASHRAE Standard is built. Figure 3-16 shows the abovementioned procedure using simple logic diagrams.

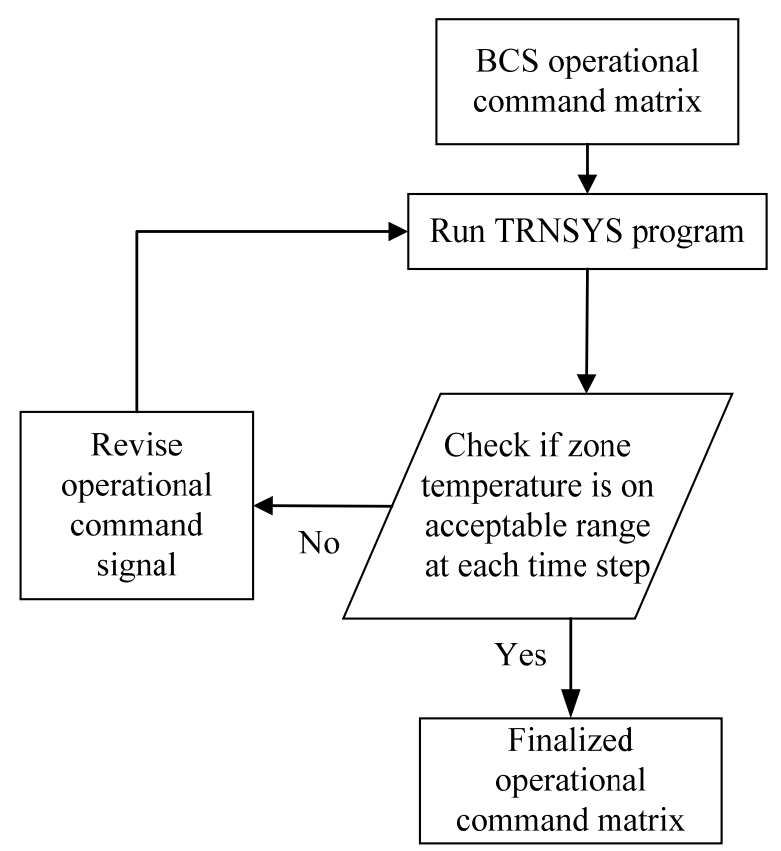

Figure 3-16: The operational mechanism of LSH-SPM model

In worst case scenario, when the outdoor temperature is cold, there is no opportunity for storing enough thermal energy inside the house because of high heat loss. Thus, the HVAC system should continuously work at peak hours to ensure thermal comfort. In such condition, there is no chance of shifting the HVAC load to off-peak hours. The same methodology is used for evening peak hours. Figure 3-17 shows the first operation command matrix (for Jan $4^{\text {th }}, 2015$ sample day) generated based on the BCS strategic plan. As Figure 3-17 shows, the ASHP ran from 6:18 am till 7:00 am and 16:21 pm till 17:00 pm to pre-heat the house and was kept off during peak hours. 


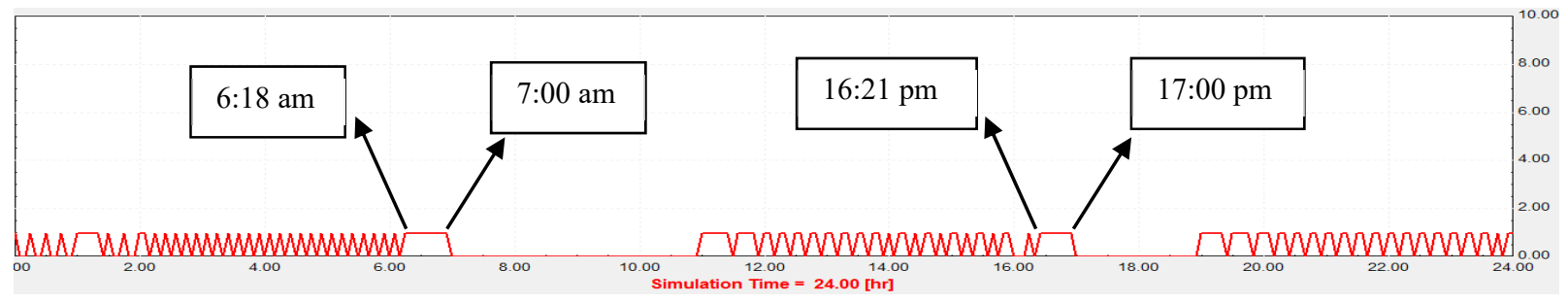

Figure 3-17: First operational command matrix generated based on BCS strategic plan

Figure 3-18 illustrates the zone temperature after running the BCS operational command matrix. As evident from Figure $3-18$, zone temperature increased to $23.4^{\circ} \mathrm{C}$ by pre-heating the house between 6:18 am and 7:00 am. However, it decreased to $16.43^{\circ} \mathrm{C}$ at $11: 00$ am (and $16.53^{\circ} \mathrm{C}$ at 19:00 pm) at the end of peak hours. In other words, HVAC system energy cost was minimized during peak hours by running the BCS operational command matrix. However, this operational command matrix led to temperature violation during this period because of the cold outdoor temperature. Since zone temperature did not fall within the ASHRAE Standard limit (between $20^{\circ} \mathrm{C}$ and $24^{\circ} \mathrm{C}$ ) during occupied hours, operational command matrix was changed with Matlab in the next iteration to bring zone temperature above the lower set point $\left(20^{\circ} \mathrm{C}\right)$ at that particular time step.

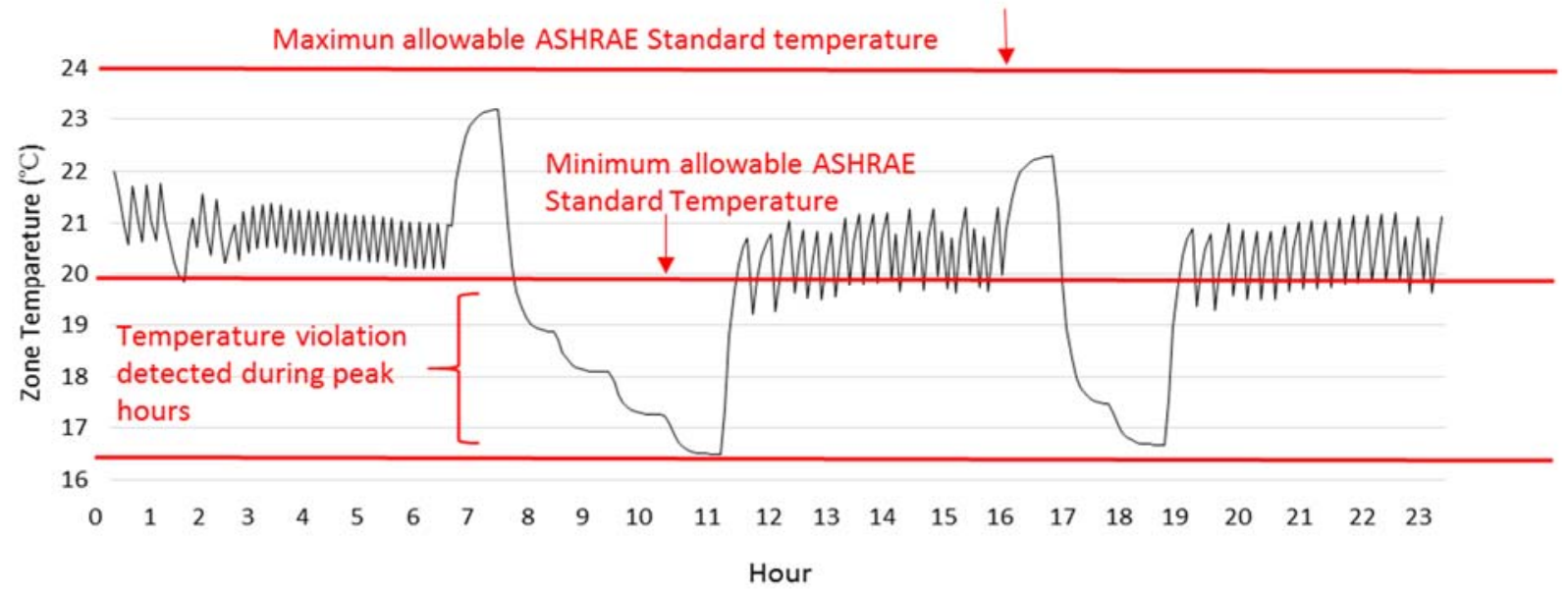

Figure 3-18: Zone Temperature after running BCS operational command matrix 


\begin{tabular}{|c|c|c|c|c|c|c|}
\hline First 6 Hours & 0 & 1 & 2 & 3 & 4 & 5 \\
\hline Command Signals & \begin{tabular}{|l|lllllllllllll}
1 & 0 & 0 & 0 & 0 & 0 & 1 & 0 & 0 & 1 & 0 & 0 \\
\end{tabular} & $\begin{array}{lllllllllllllll}1 & 1 & 1 & 1 & 1 & 0 & 0 & 0 & 0 & 1 & 0 & 0 \\
\end{array}$ & $\begin{array}{llllllllllllll}1 & 1 & 0 & 1 & 0 & 1 & 0 & 1 & 0 & 1 & 0 & 1 \\
\end{array}$ & $\begin{array}{llllllllllll}0 & 1 & 0 & 1 & 0 & 1 & 0 & 1 & 0 & 1 & 0 & 1\end{array}$ & $\begin{array}{lllllllllllll}0 & 1 & 0 & 1 & 0 & 1 & 0 & 1 & 0 & 1 & 0 & 1 \\
\end{array}$ & \begin{tabular}{lllllllll|l|l|l|l}
0 & 1 & 0 & 1 & 0 & 1 & 0 & 1 & 0 & 1 & 0 & 0 & 1 \\
\end{tabular} \\
\hline Second 6 Hours & 6 & 7 & 8 & 9 & 10 & 11 \\
\hline Command Signals & 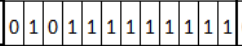 & 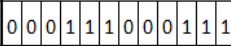 & 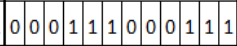 & 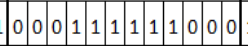 & 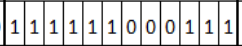 & 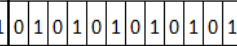 \\
\hline Third 6 Hours & 12 & 13 & 14 & 15 & 16 & 17 \\
\hline Command Signals & 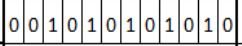 & $\begin{array}{llllllllllllll}1 & 0 & 1 & 0 & 1 & 0 & 0 & 0 & 1 & 0 & 1 & 0 \\
\end{array}$ & $\begin{array}{llllllllllllll}1 & 0 & 1 & 0 & 1 & 0 & 0 & 0 & 1 & 0 & 1 & 0 \\
\end{array}$ & 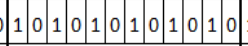 & $\begin{array}{llllllllllllll}1 & 0 & 1 & 0 & 1 & 1 & 1 & 1 & 1 & 1 & 1 & 1 \\
\end{array}$ & \begin{tabular}{lllllllll|l|l|l}
1 & 1 & 1 & 1 & 1 & 1 & 1 & 1 & 1 & 1 & 1 & 1 \\
\end{tabular} \\
\hline Fourth 6 Hours & 18 & 19 & 20 & 21 & 22 & 23 \\
\hline Command Signals & \begin{tabular}{|llllllllllllll}
1 & 1 & 1 & 0 & 0 & 0 & 1 & 1 & 1 & 1 & 1 & 1 \\
\end{tabular} & \begin{tabular}{lllllllllllll|l}
0 & 0 & 1 & 0 & 1 & 0 & 1 & 0 & 1 & 0 & 1 & 0 & 0
\end{tabular} & $\begin{array}{llllllllllll}1 & 1 & 0 & 1 & 0 & 1 & 0 & 1 & 0 & 1 & 0\end{array}$ & $\begin{array}{llllllllllllll}0 & 0 & 1 & 0 & 1 & 0 & 1 & 0 & 1 & 0 & 1 & 0 & 0\end{array}$ & $\begin{array}{llllllllllllll}1 & 0 & 1 & 0 & 1 & 0 & 1 & 0 & 1 & 0 & 1 & 0\end{array}$ & $\begin{array}{llllllllllllll}1 & 0 & 1 & 0 & 1 & 0 & 1 & 0 & 1 & 0 & 1 & 0 \\
\end{array}$ \\
\hline
\end{tabular}

Figure 3-19: Final operational command matrix generated with Matlab program

In this sample day, after ten iterations the zone temperature remained above the minimum allowable SHRAE Standard temperature at all time steps. Figure 3-19 shows the final operational command matrix.

\subsubsection{Calculating the Impact of LSH-SPM on HVAC System Daily Electricity Cost (DEC) at Different Outdoor Temperatures}

To examine the impact of LSH-SPM on House A HVAC system DEC, different simulations were run on various outdoor temperatures. Table 3-3 shows the simulation results.

Looking at Table 3-3, it is concluded that when the daily average outdoor temperature is warm (equal to or greater than $14^{\circ} \mathrm{C}$ ), the heat demand of the house is low, and therefore the ASHP is off almost the entire time. Hence, the daily cost of electricity is increased when using LSH-SPM.

The effect of LSH-SPM on the house energy system increased as the outdoor temperature decreased. The maximum saving $(17.52$ cent $)$ happened when the outdoor temperature was $6^{\circ} \mathrm{C}$ and the HVAC system remained off for 126 minutes in the morning and 67 minutes in the afternoon. At this temperature, $0.84 \mathrm{kWh}$ additional electricity was consumed during off-peak hours while $1.80 \mathrm{kWh}$ was saved during peak hours.

When the outdoor temperature was negative (for example $-2^{\circ} \mathrm{C}$ ), the DEC of HVAC system when using LSH-SPM was again higher than its DEC when no SPM was used. Figure 3-20 shows the money saved by using LSH-SPM at different outdoor temperatures.

As Figure 3-20 shows, the LSH-SPM is beneficial when the daily average outdoor temperature is between $-2^{\circ} \mathrm{C}$ and $14^{\circ} \mathrm{C}$. It is also evident that HVAC system DEC before and after using LSHSPM is increased with decreasing outdoor temperature. 
Table 3-3: Impact of LSH-SPM on House A energy system in different outdoor temperatures

\begin{tabular}{cccccc}
\hline $\begin{array}{c}\text { Average } \\
\text { Outdoor } \\
\text { Temperature } \\
\left({ }^{\circ} \text { C) }\right.\end{array}$ & $\begin{array}{c}\text { Energy } \\
\text { Cost } \\
\text { Saving } \\
(\mathbf{c})\end{array}$ & $\begin{array}{c}\text { HVAC } \\
\text { Off-time } \\
\text { Morning } \\
(\mathbf{m i n})\end{array}$ & $\begin{array}{c}\text { HVAC } \\
\text { Off-time } \\
\text { Evening } \\
(\mathbf{m i n})\end{array}$ & $\begin{array}{c}\text { Additional } \\
\text { Used Power } \\
\text { at Off-Peak } \\
\text { Hours (kWh) }\end{array}$ & $\begin{array}{c}\text { Reduced } \\
\text { Demand at } \\
\text { Peak Hours } \\
\text { (kWh) }\end{array}$ \\
\hline $\mathbf{1 4}$ & -1.94 & - & - & 0.74 & 0.53 \\
$\mathbf{1 2}$ & 4.31 & 240 & 120 & 0.63 & 1.06 \\
$\mathbf{1 0}$ & 10.05 & 213 & 120 & 0.63 & 1.58 \\
$\mathbf{8}$ & 12.10 & 186 & 93 & 0.45 & 1.69 \\
$\mathbf{6}$ & 17.52 & 126 & 67 & 0.84 & 1.80 \\
$\mathbf{4}$ & 6.34 & 93 & 33 & 0.51 & 1.14 \\
$\mathbf{2}$ & 4.77 & 33 & 15 & 0.54 & 1.07 \\
$\mathbf{0}$ & 1.62 & 24 & 6 & 0.85 & 0.42 \\
$\mathbf{- 2}$ & -14.35 & - & - & 1.19 & 0.00 \\
\hline
\end{tabular}

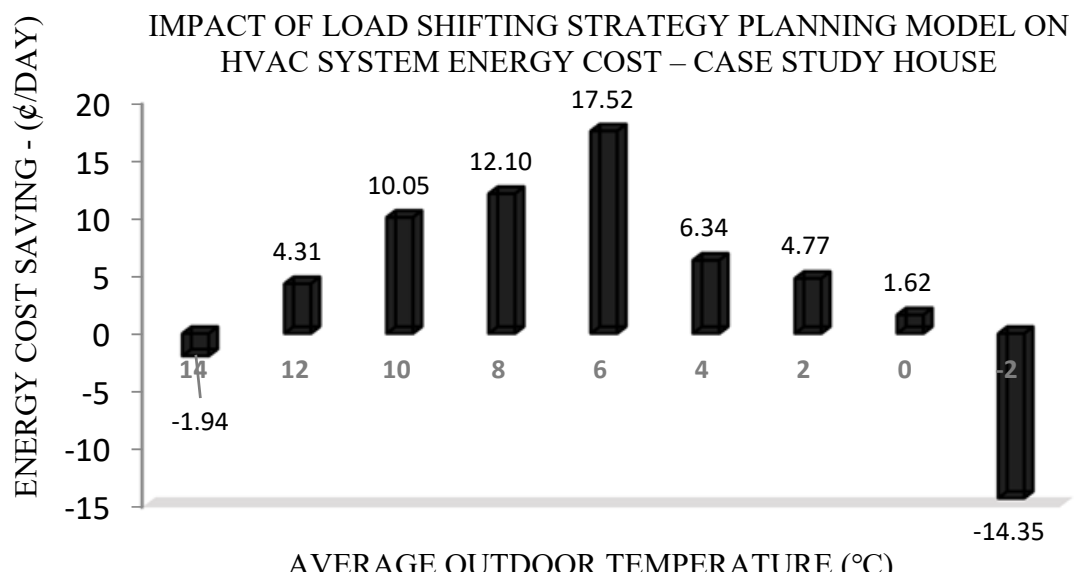

Figure 3-20: Energy cost saving using LSH-SPM at House A in different outdoor temperatures

Figure 3-21 shows the additional consumption for pre-heating the house as well as the reduced demand during peak hours for different outdoor temperatures. As Figure 3-21 shows the additional cost for pre-heating the house is greater than the saving when the outdoor temperature is higher than $14^{\circ} \mathrm{C}$ or lower than $-2^{\circ} \mathrm{C}$. 


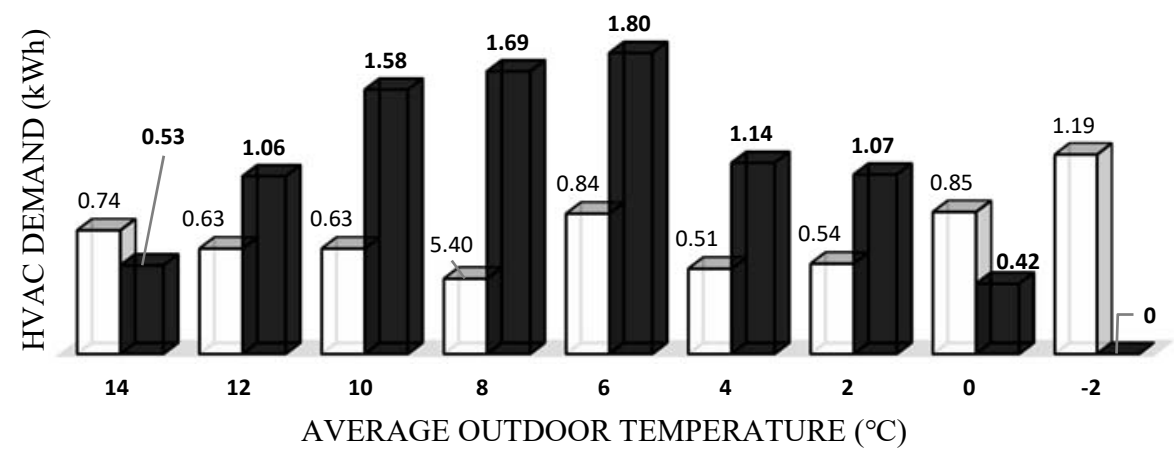

Figure 3-21: LSH-SPM additional/saving demand during different outdoor temperatures

\subsubsection{LSH-SPM Development by Integrating Smart Dual Fuel Switching System Strategy Planning Model (LSHSDFSS-SPM)}

In this scenario, the SDFSS-SPM and LSH-SPM models are combined to construct a developed intelligent strategy planning model named LSHSDFSS-SPM. This model takes advantage of both load shifting and fuel switching system to generate a novel HVAC operational command matrix. Figure 3-22 illustrates the procedures. Using this strategy planning model, not only the electrical demand of ASHP during peak hours is minimized, but also a cheaper hot air supplier system is selected in each hour. Thus, both residents and local grid benefit from such system; the residents will pay minimum fuel cost for HVAC operation while the electrical demand of the ASHP stays minimum during the peak hours.

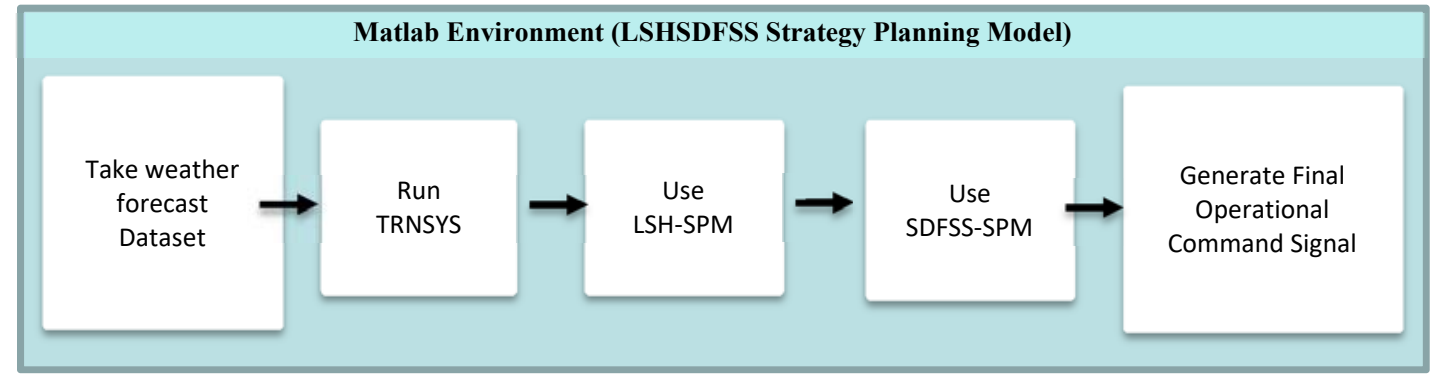

Figure 3-22: LSHSDFSS-SPM framework 
Figure 3-23 shows the operational command matrix generated after using LSHSDFSS-SPM.

\begin{tabular}{|c|c|c|c|c|c|c|}
\hline First 6 Hours & 0 & 1 & 2 & 3 & 4 & 5 \\
\hline Command Signals & A 0 O & 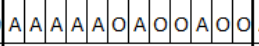 & $\begin{array}{llllllllllllll} & A & O & A & O & A & O & A & O & A & O & A \\
\end{array}$ & 0 A $O$ O & 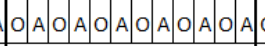 & $\begin{array}{llllllllllll} & A & O & A & O & A & O & A & O & A & O & A \\
\end{array}$ \\
\hline Second 6 Hours & 6 & 7 & 8 & 9 & 10 & 11 \\
\hline Command Signals & 0 A $O$ O & 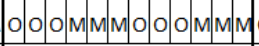 & 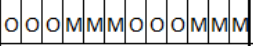 & $\mathrm{O} O \mathrm{O} \mathrm{M} \mathrm{m} \mathrm{m} \mathrm{m} \mathrm{m} \mathrm{M} \mathrm{O} O \mathrm{O}$ & 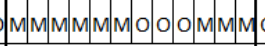 & $\mathrm{om} \mathrm{O} \mathrm{momo} \mathrm{mo} \mathrm{mo} \mathrm{m}$ \\
\hline Third 6 Hours & 12 & 13 & 14 & 15 & 16 & 17 \\
\hline Command Signals & 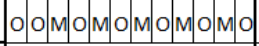 & $\mathrm{mo} \mathrm{mo} \mathrm{mo} \mathrm{mo} \mathrm{mo} \mathrm{mo}$ & $\mathrm{momo} \mathrm{mom} \mathrm{m} \mathrm{m} \mathrm{mo}$ & $\mathrm{mo} \mathrm{mo} \mathrm{mo} \mathrm{m} \mathrm{O} \mathrm{mol}$ & $\mathrm{M} O \mathrm{M} O \mathrm{M} / \mathrm{M} / \mathrm{M} / \mathrm{M} M \mathrm{M}$ & $\mathrm{M} / \mathrm{M} / \mathrm{M} / \mathrm{M} / \mathrm{M} M \mathrm{M} / \mathrm{M} / \mathrm{M}$ \\
\hline Fourth 6 Hours & 18 & 19 & 20 & 21 & 22 & 23 \\
\hline Command Signals & $\mathrm{M} / \mathrm{M} M \mathrm{M} O \mathrm{O} O \mathrm{M} / \mathrm{M} \mathrm{M} \mathrm{M} \mathrm{M} / \mathrm{M}$ & $\begin{array}{llllllllllllll} & O & A & O & A & O & A & O & A & O & A & O & 0 \\
\end{array}$ & $\begin{array}{llllllllllllllll} & A & O & A & O & A & O & A & O & A & O & A \\
\end{array}$ & 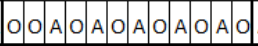 & 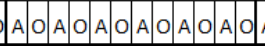 & 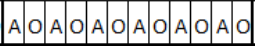 \\
\hline
\end{tabular}

Figure 3-23 : Developed operational command matrix after using LSHSDFSS-SPM

\subsection{Results and Discussion}

To demonstrate the effectiveness of Matlab-TRNSYS predictive controller, different SPMs were implemented on the system. The result of simulation before and after implementing each SPM is presented for three consecutive sample days: January $4^{\text {th }}, 5^{\text {th }}$ and $6^{\text {th }}, 2015$. Weather forecast dataset is used for estimating the house thermal demand during these days.

\subsubsection{First Sample Day (January $\left.4^{\text {th }}, 2015\right)$ Simulation Result}

\subsubsection{Baseline Case}

Table 3-4 shows the result of TRNSYS simulation. Forecast outdoor temperature, House A thermal demand, ASHP electric demand, and ASHP electricity cost are presented in the first, second, third and fourth rows of Table 3-4, respectively. The DEC of the HVAC system for this sample day is $\$ 3.61$. It should be noted that no strategy planning model has been implemented on the system yet.

Table 3-4: TRNSYS simulation result after using weather forecast dataset-baseline

\begin{tabular}{|c|c|c|c|c|c|c|c|c|c|c|c|c|c|c|c|c|c|c|c|c|c|c|c|c|c|}
\hline Hours & 0 & 1 & 2 & 3 & 4 & 5 & 6 & 7 & 8 & 9 & 10 & 11 & 12 & 13 & 14 & 15 & 16 & 17 & 18 & 19 & 20 & 21 & 22 & 23 & Sum \\
\hline $\begin{array}{c}\text { Outdoor } \\
\text { Temperature } \\
\left({ }^{\circ} \mathrm{C}\right)\end{array}$ & -4.8 & -4.6 & -5.2 & -5.4 & -5.8 & -6.0 & -6.2 & -6.3 & -6.4 & -6.9 & -7.7 & -8.7 & -9.1 & -9.4 & -9.6 & -9.8 & -9.6 & -9.5 & -9.3 & -9.2 & -9.2 & -9.1 & -8.7 & -8.4 & -- \\
\hline $\begin{array}{l}\text { Heat Demand } \\
(\mathrm{kWh})\end{array}$ & 1.44 & 1.45 & 2.91 & 2.95 & 2.99 & 3.03 & 3.06 & 3.08 & 3.09 & 3.15 & 3.27 & 3.42 & 3.47 & 3.52 & 3.55 & 3.57 & 3.55 & 3.52 & 4.08 & 4.07 & 4.07 & 4.62 & 3.98 & 3.93 & 79.77 \\
\hline $\begin{array}{l}\text { ASHP Demand } \\
\qquad(\mathrm{kWh})\end{array}$ & 0.48 & 0.47 & 0.96 & 0.97 & 0.98 & 0.99 & 0.99 & 1.00 & 1.00 & 1.01 & 1.04 & 1.08 & 1.09 & 1.10 & 1.11 & 1.11 & 1.11 & 1.10 & 1.28 & 1.27 & 1.27 & 1.45 & 1.25 & 1.24 & 25.35 \\
\hline $\begin{array}{c}\text { HVAC } \\
\text { Electricity Cost } \\
\text { (C) }\end{array}$ & 5.56 & 5.54 & 11.24 & 11.34 & 11.46 & 11.55 & 11.64 & 17.97 & 18.01 & 18.26 & 18.76 & $|16.57|$ & 16.76 & 16.93 & 17.03 & 17.08 & 17.03 & 19.80 & 22.98 & 14.91 & 14.90 & 16.96 & 14.67 & 14.53 & 361.47 \\
\hline
\end{tabular}




\subsubsection{Implementing SDFSS-SPM}

Table 3-5 demonstrates the HVAC system fuel cost after using SDFSS strategy planning model. The Daily Fuel Cost (DFC) of the HVAC system after using SDFSS-SPM is found $\$ 3.39$ which is less than the baseline case DEC (\$3.61) calculated in Section 3.3.1.1.

Table 3-5: HVAC DFC cost after using SDFSS-SPM

\begin{tabular}{|c|c|c|c|c|c|c|c|c|c|c|c|c|c|c|c|c|c|c|c|c|c|c|c|c|c|}
\hline Hours & $\mathbf{0}$ & $\mathbf{1}$ & $\mathbf{2}$ & $\mathbf{3}$ & $\mathbf{4}$ & $\mathbf{5}$ & $\mathbf{6}$ & $\mathbf{7}$ & $\mathbf{8}$ & $\mathbf{9}$ & $\mathbf{1 0}$ & $\mathbf{1 1}$ & $\mathbf{1 2}$ & $\mathbf{1 3}$ & $\mathbf{1 4}$ & $\mathbf{1 5}$ & $\mathbf{1 6}$ & $\mathbf{1 7}$ & $\mathbf{1 8}$ & $\mathbf{1 9}$ & $\mathbf{2 0}$ & $\mathbf{2 1}$ & $\mathbf{2 2}$ & $\mathbf{2 3}$ & Sum \\
\hline $\begin{array}{c}\text { Temperature } \\
\left({ }^{\circ} \mathrm{C}\right)\end{array}$ & -4.8 & -4.6 & -5.2 & -5.4 & -5.8 & -6.0 & -6.2 & -6.3 & -6.4 & -6.9 & -7.7 & -8.7 & -9.1 & -9.4 & -9.6 & -9.8 & -9.6 & -9.5 & -9.3 & -9.2 & -9.2 & -9.1 & -8.7 & -8.4 & - \\
\hline $\begin{array}{c}\text { HVAC Fuel } \\
\text { Cost }(\boldsymbol{C})\end{array}$ & 5.60 & 5.50 & 11.20 & 11.30 & 11.50 & 11.50 & 11.60 & 14.60 & 14.60 & 14.90 & 15.50 & 16.20 & 16.40 & 16.70 & 16.80 & 16.90 & 16.80 & 16.70 & 19.30 & 14.90 & 14.90 & 17.00 & 14.70 & 14.50 & 339.60 \\
\hline
\end{tabular}

\subsubsection{Implementing LSH-SPM}

Table 3-6 shows the DEC of the HVAC system after running LSH-SPM for the same sample day. The DEC of HVAC system after using LSH-SPM is found \$4.01which is higher than the HVAC energy cost (\$3.61 and \$3.39) calculated in Sections 3.3.1.1 and 3.3.1.2.

Table 3-6: HVAC DEC cost after using LSH-SPM

\begin{tabular}{|c|c|c|c|c|c|c|c|c|c|c|c|c|c|c|c|c|c|c|c|c|c|c|c|c|c|}
\hline Hours & $\mathbf{0}$ & $\mathbf{1}$ & $\mathbf{2}$ & $\mathbf{3}$ & $\mathbf{4}$ & $\mathbf{5}$ & $\mathbf{6}$ & $\mathbf{7}$ & $\mathbf{8}$ & $\mathbf{9}$ & $\mathbf{1 0}$ & $\mathbf{1 1}$ & $\mathbf{1 2}$ & $\mathbf{1 3}$ & $\mathbf{1 4}$ & $\mathbf{1 5}$ & $\mathbf{1 6}$ & $\mathbf{1 7}$ & $\mathbf{1 8}$ & $\mathbf{1 9}$ & $\mathbf{2 0}$ & $\mathbf{2 1}$ & $\mathbf{2 2}$ & $\mathbf{2 3}$ & Sum \\
\hline $\begin{array}{c}\text { Outdoor } \\
\begin{array}{c}\text { Temperature } \\
\left({ }^{\circ} \mathbf{C}\right)\end{array}\end{array}$ & -4.8 & -4.6 & -5.2 & -5.4 & -5.8 & -6.0 & -6.2 & -6.3 & -6.4 & -6.9 & -7.7 & -8.7 & -9.1 & -9.4 & -9.6 & -9.8 & -9.6 & -9.5 & -9.3 & -9.2 & -9.2 & -9.1 & -8.7 & -8.4 & -- \\
\hline $\begin{array}{c}\text { ASHP Demand } \\
(\mathbf{k W h})\end{array}$ & 0.48 & 0.47 & 0.96 & 0.97 & 0.98 & 0.99 & 1.66 & 1.00 & 1.00 & 1.01 & 1.56 & 1.08 & 1.09 & 1.10 & 1.11 & 1.11 & 1.84 & 2.20 & 1.64 & 0.91 & 1.09 & 1.09 & 1.07 & 1.06 & $\mathbf{2 7 . 4 7}$ \\
\hline $\begin{array}{c}\text { HVAC } \\
\text { Electricity Cost } \\
(\boldsymbol{C})\end{array}$ & 5.60 & 5.50 & 11.20 & 11.30 & 11.50 & 11.50 & 19.40 & 18.00 & 18.00 & 18.30 & 28.10 & 16.60 & 16.80 & 16.90 & 17.00 & 17.10 & 28.40 & 39.60 & 29.50 & 10.60 & 12.80 & 12.70 & 12.60 & 12.50 & 401.50 \\
\hline
\end{tabular}

\subsubsection{Implementing LSHSDFSS-SPM}

After running the model by LSHSDFSS-SPM, the DFC of HVAC system (calculated based on Table 3-7) for the same sample day is found to be $\$ 3.73$.

Table 3-7: HVAC DFC cost after using LSHSDFSS-SPM

\begin{tabular}{|c|c|c|c|c|c|c|c|c|c|c|c|c|c|c|c|c|c|c|c|c|c|c|c|c|c|}
\hline Hours & $\mathbf{0}$ & $\mathbf{1}$ & $\mathbf{2}$ & $\mathbf{3}$ & $\mathbf{4}$ & $\mathbf{5}$ & $\mathbf{6}$ & $\mathbf{7}$ & $\mathbf{8}$ & $\mathbf{9}$ & $\mathbf{1 0}$ & $\mathbf{1 1}$ & $\mathbf{1 2}$ & $\mathbf{1 3}$ & $\mathbf{1 4}$ & $\mathbf{1 5}$ & $\mathbf{1 6}$ & $\mathbf{1 7}$ & $\mathbf{1 8}$ & $\mathbf{1 9}$ & $\mathbf{2 0}$ & $\mathbf{2 1}$ & $\mathbf{2 2}$ & $\mathbf{2 3}$ & $\mathbf{S u m}$ \\
\hline $\begin{array}{c}\text { Outdoor } \\
\begin{array}{c}\text { Temperature } \\
\left({ }^{\circ} \mathbf{C}\right)\end{array}\end{array}$ & -4.8 & -4.6 & -5.2 & -5.4 & -5.8 & -6.0 & -6.2 & -6.3 & -6.4 & -6.9 & -7.7 & -8.7 & -9.1 & -9.4 & -9.6 & -9.8 & -9.6 & -9.5 & -9.3 & -9.2 & -9.2 & -9.1 & -8.7 & -8.4 & -- \\
\hline $\begin{array}{c}\text { HVAC Fuel } \\
\text { Cost }(\boldsymbol{C})\end{array}$ & 5.60 & 5.50 & 11.20 & 11.30 & 11.50 & 11.50 & 19.40 & 14.60 & 14.60 & 14.90 & 23.20 & 16.20 & 16.40 & 16.70 & 16.80 & $\mathbf{1 6 . 9 0}$ & 28.00 & 33.40 & 24.80 & 10.60 & 12.80 & 12.70 & 12.60 & 12.50 & 373.70 \\
\hline
\end{tabular}




\subsubsection{Comparing the Results of Different SPMs - First Sample Day}

Figure 3-24 shows the potential energy cost savings on the first sample day at House A as the result of using different SPMs. The baseline daily energy cost of the HVAC system when no strategy planning model was applied to the system was $\$ 3.61$. Using SDFSS-SPM the daily energy cost decreased to $\$ 3.39$. However, the HVAC energy cost increased to $\$ 4.01$ when LSH-SPM was used. This was due to the fact that the outdoor temperature on the sample day was very cold and out of the temperature range that allows the system to benefit from LSH-SPM. Using LSHSDFSSSPM, the HVAC energy cost increased to $\$ 3.73$. Figure 3-24 shows that SDFSS allowed maximum saving during the sample day.

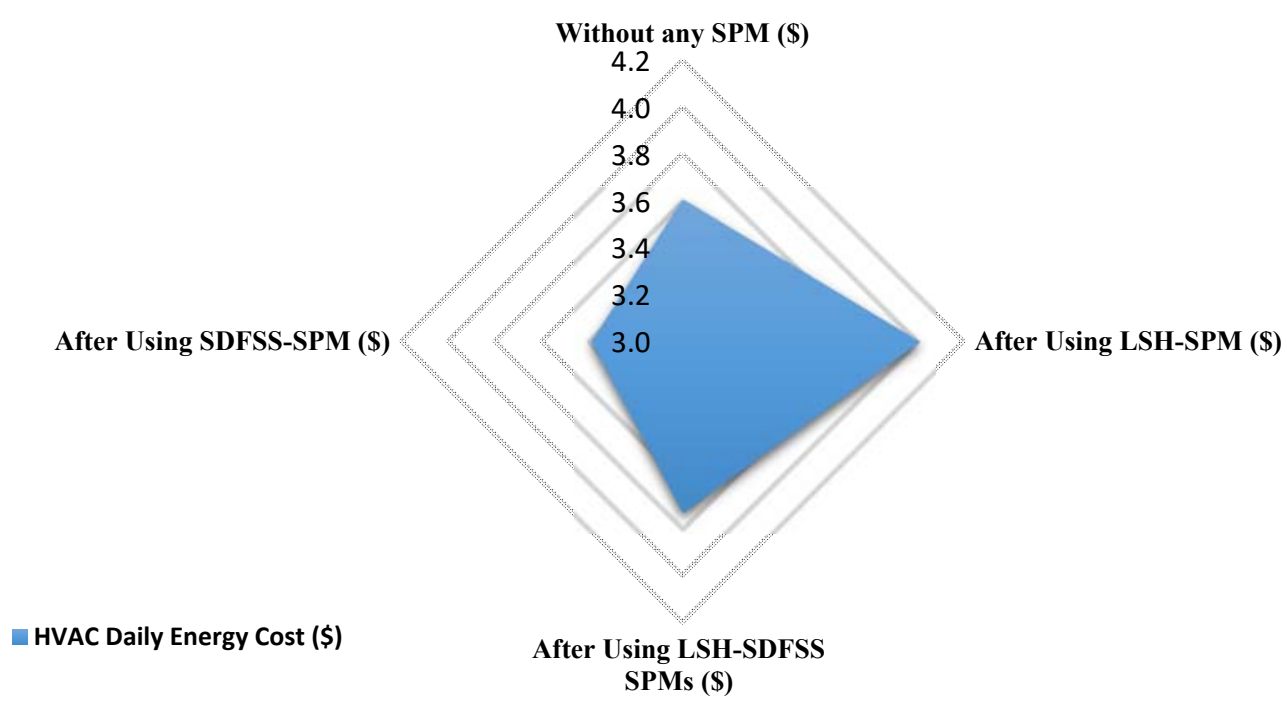

Figure 3-24: Comparing the potential energy cost of different SPMs on first sample day

\subsubsection{Comparing the Results of Different SPMs - Second Sample Day, January $5^{\text {th }}$}

Figure 3-25 shows the outdoor temperature on January $5^{\text {th }}$. 


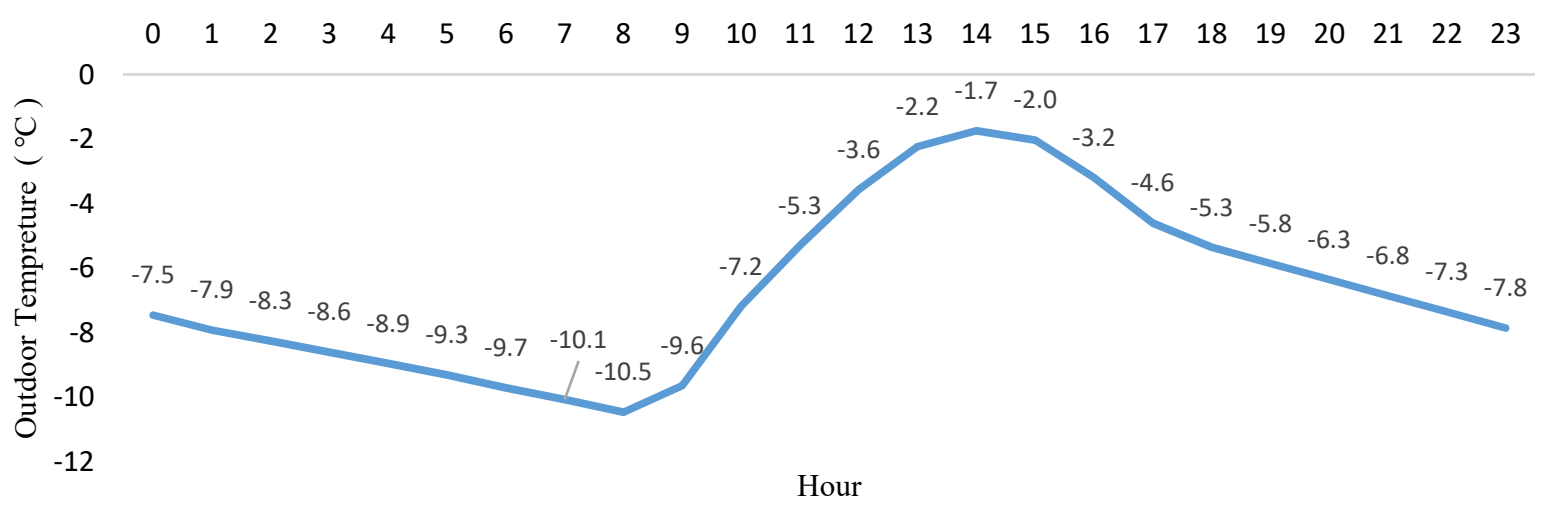

Figure 3-25: Outdoor temperature on January $5^{\text {th }}, 2015$

Figure 3-26 shows the potential energy cost savings on the second sample day at house A as the result of using different SPMs. The baseline daily energy cost of the HVAC system when no strategy planning model was applied to the system was \$3.44. Using SDFSS-SPM the daily energy cost decreased to $\$ 3.23$. However, the HVAC energy cost increased to \$3.86 when LSH-SPM was used. This was due to the fact that the outdoor temperature on January $5^{\text {th }}$ was also cold and out of the range that allows the system to benefit from LSH-SPM. Using LSHSDFSS-SPM, the HVAC energy cost increased to $\$ 3.60$. Figure 3-26 shows that SDFSS allowed maximum saving during the second sample day.

After Using SDFSS-SPM (\$)

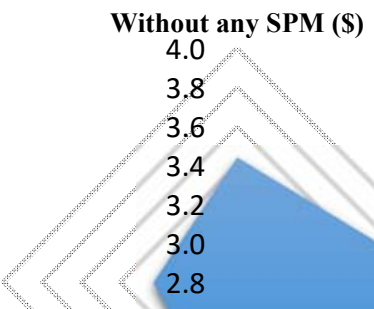

After Using LSH-SDFSS SPMs (\$)

Figure 3-26: Comparing the potential energy cost of different SPMs on the second sample day 


\subsubsection{Comparing the Results of Different SPMs - Third Sample Day, January $6^{\text {th }}$}

Figure 3-27 shows the outdoor temperature on January $6^{\text {th }}$. Figure 3-28 shows the potential energy cost savings on the third sample day at house A as the result of using different SPMs. The baseline daily energy cost of the HVAC system when no strategy planning model was applied to the system was $\$ 4.55$. Using SDFSS-SPM the daily energy cost decreased to $\$ 3.85$. On this sample day, the HVAC energy cost increased to $\$ 5.13$ when LSH-SPM was used. This was due to the fact that the outdoor temperature on January $6^{\text {th }}$ was also out of the range that allows the system to benefit from LSH-SPM. Using LSHSDFSS-SPM, the HVAC energy cost decreased to \$4.26. Figure 3-28 shows that SDFSS allowed maximum saving during the third sample day.

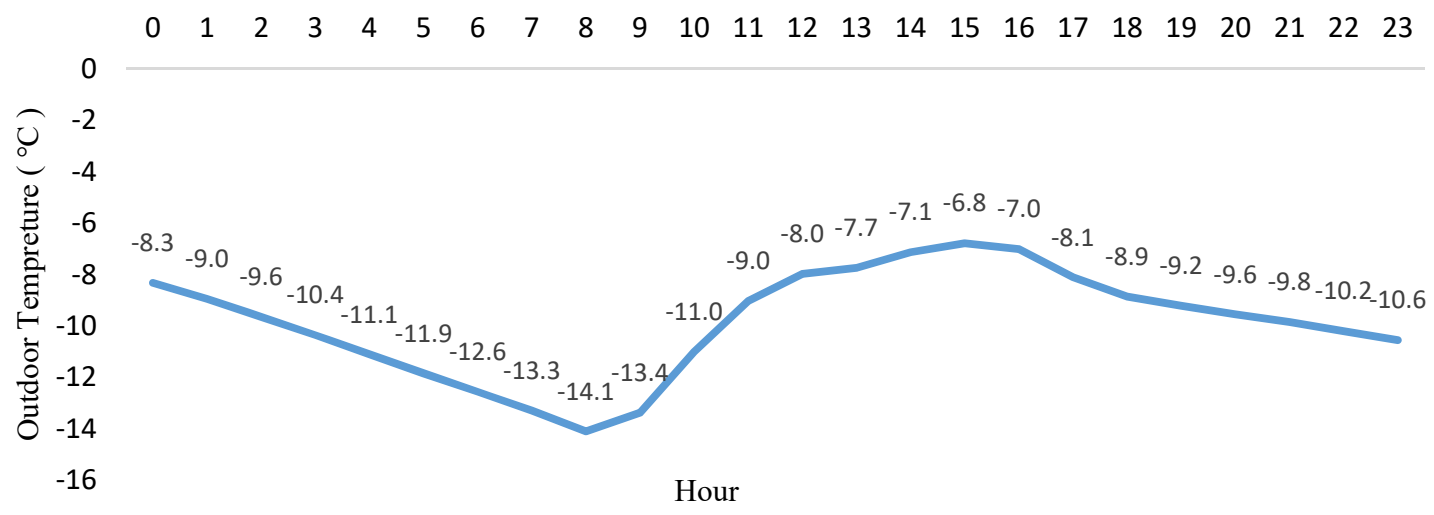

Figure 3-27: Outdoor temperature on January $6^{\text {th }}, 2015$

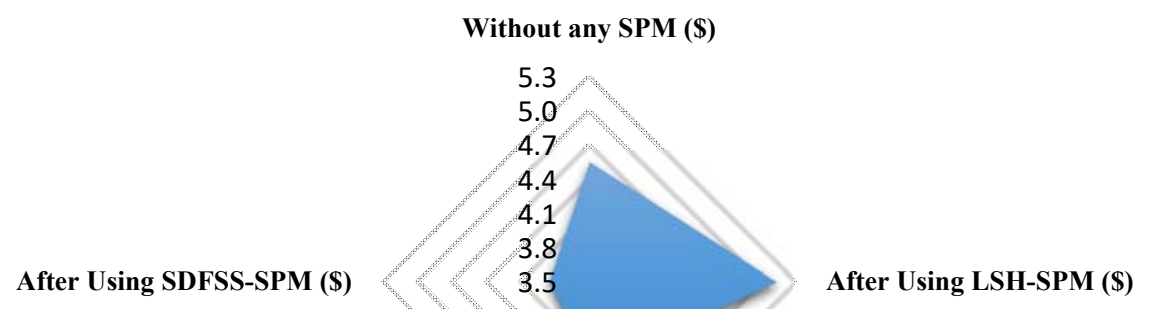

HVAC Daily Energy Cost (\$)

After Using LSH-SDFSS SPMs (\$)

Figure 3-28: Comparing the potential energy cost of different SPMs on the third sample day 


\subsubsection{Effectiveness of Various SPMs During Different Outdoor Temperatures}

SDFSS-SPM was the first strategy planning model applied for selecting the least expensive hotair supplier system at each hour. This SPM could notably reduce the owners' overall HVAC energy cost. In addition to reducing the energy cost, this SPM model manages HVAC energy demand by consuming less electricity during the peak and mid-peak hours. Based on the simulation result presented in Section 3.2.3, when the outdoor temperature is above $0^{\circ} \mathrm{C}$ there is no opportunity for taking advantage of SDFSS-SPM. However, when the outdoor temperature is below $0^{\circ} \mathrm{C}$, the DFC of HVAC system decreases using this model. As the outdoor temperature gets colder, more saving takes place. The second strategy planning model used in this project was LSH-SPM model. This control strategy shifts the HVAC load from peak to off or mid-peak hours. This grid-friendly SPM model reduces local grid overloading by decreasing the HVAC energy demand during peak hours. Since outdoor temperature and house characteristics directly impact the thermal energy stored in a house, a smart method is used to determine the best starting time for pre-heating the house. This method not only ensures the thermal comfort, but also minimizes the electricity consumed by the ASHP to pre-heat the house. Based on the simulation results presented in Section 3.2.4, when the outdoor temperature is between $-2^{\circ} \mathrm{C}$ and $14^{\circ} \mathrm{C}$, the best results are obtained by implementing the LSH-SPM model. When the outdoor temperature is above $14^{\circ} \mathrm{C}$, the potential cost saving achieved during the peak hours is less than the additional energy cost related to pre-heating the house during off-peak hours. Additionally, when the outdoor temperature is below $0^{\circ} \mathrm{C}$, there is no chance for storing enough thermal energy in the house (based on our light thermal mass test case house) to keep the HVAC system off during peak hours.

LSHSDFSS-SPM was the third strategy planning model examined and was developed by integrating SDFSS-SPM and LSH-SPM models into a single system. LSHSDFSS-SPM takes advantage of both load shifting and fuel switching system. LSHSDFSS-SPM allows for maximum cost saving when the outdoor temperature changes between $-2^{\circ} \mathrm{C}$ and $0^{\circ} \mathrm{C}$.

\subsection{Conclusion}

Different powerful house energy simulators such as TRNSYS, EnergyPlus, Mathcad, and esp-r have been used by the researchers for simulating house and building energy systems. These 
simulators offer an excellent opportunity for detailed design and modeling of a house and its HVAC system and provide very accurate simulation results useful for performance analysis and optimization process. However, these simulators do not include sub-models of advanced devices/strategies for controlling HVAC system operation and suffer from poor control mechanism. In addition to the lack of advanced controllers, the aforementioned simulators mostly use operational/historical weather dataset (given in a library file) for simulating the house energy system. Hence, they inherently offer no mechanism for estimating the future state of their process models based on the forecast weather dataset. Therefore, no predictive controller can be designed and run within these simulators. In this project, a Matlab-TRNSYS co-simulator was developed to control/manage the TRNSYS program as one of the most powerful house energy simulators with an advanced predictive controller. This co-simulator can be utilized as a test bed for implementing different SPMs. To show the effectiveness of this co-simulator which acts as a smart grid-friendly controller, three distinct strategy planning models were developed and applied in order to manage the hourly load demand of an HVAC system on the upcoming 24 hours. SDFSS-SPM was the first strategy planning model employed for selecting the least expensive hot-air supplier system at each hour. Simulation results on three consecutive sample days show that this SPM could bring a total of $\$ 1.14(9.2 \%)$ saving in the house's HVAC energy cost. In addition to reducing the energy cost, this SPM model manages HVAC demand by consuming less electricity during the peak hours. The second strategy planning model developed in this project was LSH-SPM model. LSH-SPM model shifts the HVAC load from peak to off- or mid-peak hours. This grid-friendly SPM model is aimed to reduce overloading on the local grid by decreasing the demand during peak hours. Due to very cold outdoor temperature on the sample days, LSH-SPM increased HVAC energy cost by $\$ 1.42$ $(10.7 \%)$ in total. LSHSDFSS-SPM was the third strategy planning model investigated. LSHSDFSS-SPM was developed by integrating SDFSS-SPM and LSH-SPM models. LSHSDFSS-SPM takes advantage of both load shifting and fuel switching system. LSHSDFSSSPM increased the HVAC energy cost on the first and second sample days due to the very cold outdoor temperature during these days. However, HVAC energy cost decreased by applying LSHSDFSS-SPM on the third sample day due to the effect of SDFSS-SPM. In total, LSHSDFSSSPM decreased HVAC energy cost by $\$ 0.01(0.3 \%)$. 


\subsection{References}

3.1. Mathew, V., Sitaraman, R.K., Shenoy, P., "Reducing energy costs in Internet-scale distributed systems using load shifting", Communication Systems and Networks (COMSNETS), Sixth International Conference, Bangalore, 2014. p. $1-8$.

3.2. Liu, Y., Yuen, C., Member, S., "Peak-to-Average Ratio Constrained Demand-Side Management With Consumer's Preference in Residential Smart Grid", Selected Topics in Signal Processing, IEEE Journal, 2014.8(6): p. 1084 - 1097.

3.3. Radhakrishnan, A, Selvan, M.P., "Load scheduling for smart energy management in residential buildings with renewable sources" , Power Systems Conference (NPSC), 2014 Eighteenth National ,Guwahati, 2014. p. $1-6$

3.4. Afram, A, Janabi-Sharifi, F, "Gray-box modeling and validation of residential HVAC system for control system design”, Applied Energy, 2015. (137): p. 134-150.

3.5. Ma, J, Qin, J, Salsbury, T, Xu, P, "Demand reduction in building energy systems based on economic model predictive control”, Chemical Engineering Science, 2011. 67(1): p. 92

3.6. Candanedo, J, Athienitis, A, "Predictive control of radiant floor heating and solar-source heat pump operation in a solar house", HVAC\&R Research, 2011. 17(3): p. 235-256.

3.7. Naidu, D.S, Craig, G.R, "Advanced control strategies for heating, ventilation, airconditioning, and refrigeration systems - An overview: Part I” ,Hard control. HVAC R Res, 2011. 17(1): p. 2-21.

3.8. Weiss, M.V.G., “Adaptive neuro energy management control strategies for HVAC systems in buildings, in Build" , Civ and Environ Eng. 2006, Concordia University: Montreal, Quebec, Canada.

3.9. Platt, G, Ward, J, Wall, J, "Optimal supervisory HVAC control: Experiences in Australia", HVAC R Res, 2011. 17(3): p. 297-308.

3.10. Srinivas, K, Ning, L, "Evaluation of Residential HVAC Control Strategies for Demand Response Programs”, ASHRAE Trans, 2006. 112: p. 535-546.

3.11. García-Domingo, B., Torres-Ramírez, M., Casa, J., Aguilera, J., "Design of the back-up system in Patio 2.12 photovoltaic installation”, Energy and Buildings, 2014. 83: P 130-139.

3.12. Keshtkar, A, Arzanpour, S, Ahmadi, P, "Smart residential load reduction via fuzzy logic, wireless sensors, and smart grid incentives", Energy and Buildings, 2015. 104: p 165-180.

3.13. Onda, H., Yamamoto, S., Takeshit, H., Okamoto, S., "Peak Load Shifting and Electricity Charges Reduction Realized by Electric Vehicle Storage Virtualization”, AASRI Procedia, 2014. 7: p 101-106.

3.14. Boehm, R.F., "An approach to decreasing the peak electrical demand in residences”, Energy Procedia, 2012. 14: p 337-342.

3.15. Castillo-Cagigal, M., Gutierrez, A., Monastrieo, F., Caamano, E., Masa, D., “A semidistributed electric demand-side management system with PV generation for selfconsumption enhancement", Energy Conversion and Management, 2011. 7: p 2659-2666.

3.16. Fernandes, F., Morais, H., Vale, Z.," Dynamic load management in a smart home to participate in demand response events", Energy and Buildings, 2014. 82: p 592-606. 
3.17. Beizaee, A, Allison, D., Lomas, K., Foda, E., Loveday, D., "Measuring the potential of zonal space heating controls to reduce energy use in UK homes: The case of un-furbished 1930s dwellings", Energy and Buildings, 2015. 92: p 29-44.

3.18. H.F. Naspolini, H.S.G. Militão, R. Rüther, "The role and benefits of solar water heating in the energy demands of low-income dwellings in Brazil", Energy Conversion and Management, 2010. 51: p 2835-2845.

3.19. Chassin, DP., Stoustrup, J., Agothoklis, P., Djilali, N., "A new thermostat for real-time price demand response: Cost, comfort and energy impacts of discrete-time control without deadband", Applied Energy, 2015. 155: p 816-825.

3.20. Li, J., Poulton, G., Platt, G., Wall, j., James, G., “ Dynamic zone modelling for HVAC system control", Int J of Model, Identif and Control, 2010. 9(1/2): p. 5-13.

3.21. Nielsen, T.R., Drivsholm, C., "Energy efficient demand controlled ventilation in single family houses", Energy and Buildings, 2010. 42: p 1995-1998.

3.22. Gulden, C.C.S.G., "Optimization of HVAC control strategies by building management systems case study: Özdilek shopping center in Energy Engineering” ,. 2003, Izmir Institute of Technology: Izmir, Turkey.

3.23. Hart, R., "Advanced unitary HVAC control sequence", ASHRAE Transactions, 2012. 118(1): p. 628.

3.24. Reid, H., Callahan, J., Anderson, K., Johanning, P., "Unitary HVAC Premium Ventilation Upgrade", ASHRAE Transactions, 2011. 117(1): p. 517.

3.25. Al-Rabghi, O.M. and M.M. Akyurt, "A survey of energy efficient strategies for effective air conditioning", Energy Conversion and Management, 2004. 45(11): p. 1643-1654.

3.26. Pal, A.K. and R.K. Mudi, "Self-Tuning Fuzzy PI Controller and its Application to HVAC Systems", Int. J. Computer Cognition, 2008. 6(1): p. 25-30.

3.27. Bansal, R.C. and J.C. Pandey, "Load forecasting using artificial intelligence techniques: a literature survey" Int. J. Computer Applications in Technology, 2005. 22(2/3): p. 109-119.

3.28. Kulkarni, M.R. and F. Hong, "Energy optimal control of a residential space-conditioning system based on sensible heat transfer modeling", Building and Environment, 2004. 39: p. 31-38.

3.29. Ouden, C., "Buildings thermal analysis, an electronic mathcad book: A.K. Athienitis, MathSoft, Cambridge, USA", Solar Energy, 1997. 60: p 61.

3.30. Wills, A., Cynthia A. Cruickshank, Ian Beausoleil-Morrison," Application of the ESPr/TRNSYS Co-Simulator to Study Solar Heating with a Single-House Scale Seasonal Storage", Energy Procedia, 2012. 30: p 715-722.

3.31. Justin C. DeBlois, Melissa M. Bilec, Laura A. Schaefer," Design and zonal building energy modeling of a roof integrated solar chimney", Renewable Energy, 2013. 52: p 241-250.

3.32. Afram, A., Janabi-Sharifi, F., "Theory and application of HVAC control systems - A review of model predictive control (MPC)", Building and Environment, 2014. 72: p. 343-355.

3.33. http://dd.weatheroffice.ec.gc.ca. (Jan 4th, 2015). 
3.34. Oldewurtela, F., Parisio, A., Jones, C., Gyalistras, D., Stauch, V., "Use of model predictive control and weather forecasts for energy efficient building climate control", Energy and Buildings, 2012. 45: p. 15-27

3.35. Poulin, L., "Weather Forecast Data An Important Input into Building Management Systems", National Prediction Operations Division, ICEBO 2013, Montreal, Qc. October, 2013.

3.36. Kim, S., Augenbroe, G., "Using the National Digital Forecast Database for model-based building controls", Automation in Construction, 2012. 27: p. 170-182

3.37. Poulin, L., "An NWP forecast information matrix in support of renewable energy applications", CMOS 2013, Saskatoon

3.38. Vernaya, C., Pitavala, S., Blancb, P. "Review of satellite-based surface solar irradiation databases for the engineering, the financing and the operating of photovoltaic system", Energy Procedia, 2014. 57: p. 1383 - 1391.

3.39. Rodriguez, A., antonio, J., Pozo, D., Tovar, J., “An artificial neural network ensemble model for estimating global solar radiation from Meteosat satellite images", Energy, 2013. 61: p. 636- 645 .

3.40. Fung, A., Zhang, D., "The Archetype Sustainable Houses: Overview of Design and Monitoring Systems", Toronto and Region Conservation's Sustainable Technologies Evaluation Program, June 2011.

3.41. Safa, A., Fung, A., Kumar, R., "Performance of two-stage variable capacity air source heat pump: Field performance results and TRNSYS simulation", Energy and Buildings, 2015. 94: p. 80-90.

3.42. Dembo, A., Fung, A.S., Ng, K.L.R., Pyrka, A., "The archetype sustainable house: investigating its potentials to achieving the net-zero energy status based on the results of a detailed energy audit, in: Proceedings of the 1st International High Performance Buildings Conference", 2010. p. 1-8.

3.43. Zhang, D., Barua, R., Fung, A.S., “ TRCA-BILD archetype sustainable house-overview of monitoring system and preliminary results for mechanical systems, ASHRAE Trans, 2011. 117 (2): p. 597-612.

3.44. VIESMANN, Technical Data Manual, Gas-fired wall-mounted condensing boiler 12 to 285 MBH (3.5 to $83.5 \mathrm{~kW})$, Vitodens 200-W Series B2HA.

3.45. https://en.wikipedia.org/wiki/Geography_of_Toronto. (Oct $\left.6^{\text {th }}, 2015\right)$.

3.46. https://en.wikipedia.org/wiki/List_of_extreme_temperatures_in_Canada. (Oct $6^{\text {th }}$, 2015).

3.47. ASHRAE 2009, ASHRAE Handbook-Fundamentals, American Society of Heating, Refrigeration and Air-Conditioning Engineers, Inc., Atlanta, GA.

3.48. Crarley, D., Hand, J., Kummert, M., \& Griffith, "Contrasting the Capabilities of Building Energy Perfromance Simulation Programs", 2005, Washington , United States of America.

3.49. Klein, S., Beckman, W., Mitchell, J., Duffie, J., Duffie, N., Freeman, T., "TRNSYS 16 - A Transient System Simulation Program”, 2006, Madison, Wisconsin, U.S.A. 
3.50. Kamel, R., Fung, A.S., "Modeling, simulation and feasibility analysis of residential BIPV/T+ASHP system in cold climate-Canada", Energy and Buildings, 2014. 82: p. 758770. 


\section{Chapter 4: Effects of Novel Energy Conservation Strategy Planning Models on HVAC System Energy Demand/Cost in the Heating and Cooling Seasons}

The effects of novel energy conservation strategy planning models (that have been developed in chapter 3) on HVAC system energy demand/cost will be investigated in this chapter in heating and

cooling seasons using Matlab-TRNSYS Co-simulator. This chapter covers the contents of the second journal paper that was published in the Journal of Applied Energy.

Since the novel Load Shifting (LSH) strategy planning model only covered the heating season, LSH model has been developed to further include/cover the cooling (summer) season using precooling process. Ontario 2015 time of use pricing has been used in this chapter for calculating the energy cost savings. Simulation results showed that in the heating season, the operating/energy cost of HVAC system decreased significantly (23.8\%) by implementing SDFSS-SPM. LSHSDFSS-SPM reduced the HVAC system operating cost by $15.8 \%$. In the cooling season, LSH-SPM reduced the HVAC system operating cost by $6.63 \%$.

\subsection{Introduction}

Residential Houses/buildings (RHs) must be seen as significant elements of a larger, dynamic network of energy system. Therefore, a network of energy system is significantly affected by the behavior of RHs [4.1, 4.2]. For example, based on Pagani and Aiello [4.3] energy model simulation result, disruption in demand management can be detrimental to energy systems. In contrast, as Siano and Sarno [4.4] shown in simulation result, RHs enable to improve the management of energy network. The network energy saving is improved significantly using the distribution locational marginal price (D-LMP) model developed in [4.4]. What makes the RHs' roles prominent in managing the energy network, is their energy structure. They are capable of storing thermal energy that results in managing their energy demand. Arteconi et al. [4.5] have investigated the load shifting potentials of thermal energy storage (TES) in a residential building. The simulation result showed energy cost saving using on and off peak tariffs. Thermal energy storage and energy demand management can be achieved by employing smart strategy planning models (SPMs) in the HVAC system as one of the largest energy consumers in RHs/buildings. In 
Vakiloroaya et al. [4.6] review paper, different demand management and energy saving SPMs for typical residential HVAC systems are described in detail and compared. Huang et al. [4.7] showed the advantage of different energy saving SPMs such as load shifting in a HVAC predictive energy model. The effects of different energy conservation SPMs such as daily optimal deadband and daily optimal set point are investigated in [4.8] using EnergyPlus program. Kim et al. [4.9] have developed a daylighting meta-model that has been integrated into a HVAC system to take the maximum advantage of daylighting for preparing thermal comfort. This meta-model offered an average of $13.7 \%$ energy saving. Different heat gain reduction methodologies/SPMs have been developed in [4.10]. Based on this paper calculation, more than $75 \%$ of building heat gain were generated by solar heat gain and lighting system. By managing these heat gains, more than $45 \%$ saving on HVAC system energy cost was achieved. Christantoni et al. [4.11] used EnergyPlus simulation model to implement different demand response SPMs for shifting the building electrical demand. In this research, contribution of HVAC system and building capacitance was evaluated using demand response SPMs. In [4.12] research, energy saving potentials of various set point strategies were investigated in a museum. Using these strategies led to $77 \%$ improvement in thermal comfort while decreased $82 \%$ of HVAC system energy demand. Wang et al. [4.13] have modeled the influence of occupants and its essential effects on building performance by generating mean profiles of occupancy variables in order to increase HVAC system energy efficiency. The role of residential heat pumps and load shifting, and their contribution in network operational cost and $\mathrm{CO}_{2}$ emission reduction has been investigated in [4.14]. As Beizaee et al. [4.15] have shown, the thermal demand of a house can be decreased using zonal space heating SPM. This SPM could reduce the natural gas consumption by $11.2 \%$ in only eight weeks. Chassin et al. [4.16] investigated the impacts of discrete-time SPM on a residential house HVAC system and reported up to $25 \%$ reduction in HVAC system energy demand. A dynamic zone modelling system as an energy conservation SPM was developed by Li et al. [4.17] to reduce the HVAC system energy cost. Different ingenious methods like using tokens (as a surrogate for thermal demand) [4.18] and demand response potentials of high-raised building ventilation fans [4.19] have been used as smart SPMs to conserve the network energy.

In addition to supporting the network of energy system, lower overall energy cost could be achieved for the homeowners with such intelligent SPMs. Hence, as Di Giorgio and Liberati [4.20] concluded, both energy consumers and local grid benefit from such intelligent planning models. 
Regardless of the fact that numerous research with different criteria have been previously conducted for designing different strategy planning models for residential HVAC systems with the aim of reducing demand and energy cost, less attention has been paid to energy conservation SPMs that use combination of fuels/energies for running the HVAC system. Furthermore, matching and tuning SPMs with the real-time dynamic characteristics of the house thermal model and HVAC system model as comprehensive process models has been poorly noted before.

Thus, in this chapter, novel strategy planning models that have been developed in Chapter 3 including: 1) Smart Dual Fuel Switching System (SDFSS), 2) Load Shifting (LSH), and 3) LSHSDFSS model as the combination of fuel switching and load shifting strategy planning models have been implemented in House A HVAC system. The behavior of HVAC system is numerically simulated in-depth during winter (heating) and summer (cooling) seasons. Total savings in the HVAC system energy cost are calculated in this chapter by implementing developed SPMs in winter and summer seasons.

\subsection{Model Description}

\subsubsection{Methodology}

The simulation is started by running case study house TRNSYS model for the heating and cooling seasons in order to generate the baseline data. The weather file used to calculate the house's thermal demand is the metropolitan Toronto weather given in the TRNSYS library. Different operational command matrices are generated by Matlab MPC controller (according to the baseline data) in order to implement various SPMs on the house's HVAC system. TRNSYS model is run considering the generated operational command matrices in simulation process. The data generated by implementing each strategy planning model is saved for the further post processing.

\subsubsection{Electricity Prices}

Distribution System Operators (DSOs) exert rates that penalize energy use on peak hours via Timeof-Use (TOU) and/or demand charges rates. Figure 4-1 shows the electricity price in Ontario as of November $1^{\text {st }}, 2015$ for summer and winter seasons. In this chapter, $13.30 \notin / \mathrm{kWh}, 17.80 \notin / \mathrm{kWh}$ and $22.60 \phi / \mathrm{kWh}$ are used as the electricity prices for off-peak, mid-peak and peak hours, respectively. Ontario Energy Board (OEB) TOU electricity prices were used to estimate these 
prices. One of the most important factors that adds value in the developed SPMs is the annual increment in TOU electricity prices. In other words, the potential energy cost saving of developed SPMs would be increased annually by raising the rate of TOU electricity prices. The increment rates of TOU electricity prices are calculated comparing 2014 and 2015 TOU electricity prices [4.21] and shown in Table 4-1.

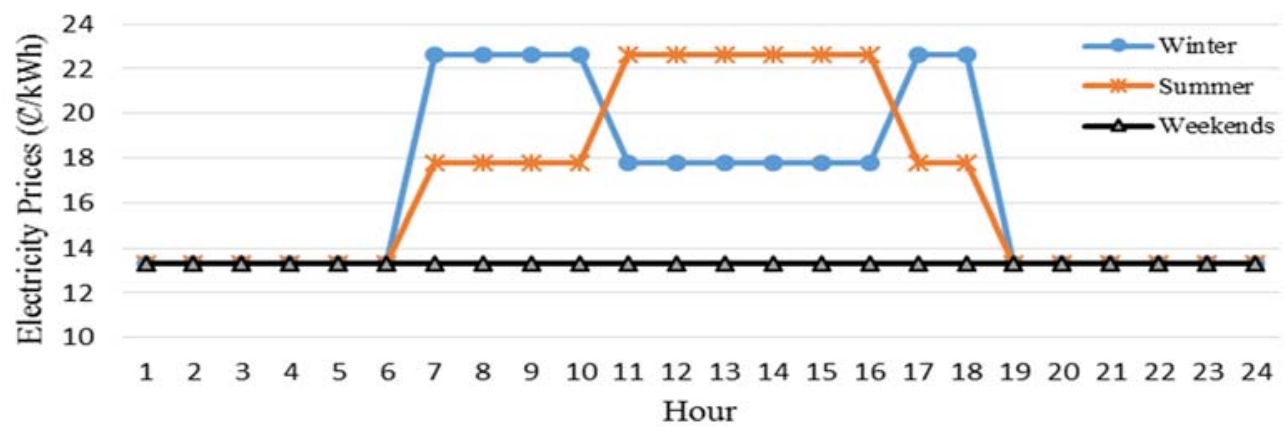

Figure 4-1: Price of electricity in Ontario as of November 1st, 2015 for winter and summer seasons

Table 4-1: Compering the increment rates of TOU electricity prices

\begin{tabular}{|c||c|c|c|}
\hline \multicolumn{5}{|c|}{ TOU Electricity Prices (d/kWh) } \\
\hline \hline Year & Off-peak & Mid-peak & Peak \\
\hline 2014 & 11.7 & 15.4 & 18.0 \\
\hline 2015 & 13.3 & 17.8 & 22.6 \\
\hline Increment Rates & $12.0 \%$ & $13.4 \%$ & $20.3 \%$ \\
\hline
\end{tabular}

Table 4-2 shows the increment percentages of electricity prices between off-peak and peak hours in 2014 and 2015. Since in 2015 there is $6 \%$ more difference between electricity prices between off-peak and peak hours, LSH-SPM is more beneficial in terms of energy cost saving.

Table 4-2: Incremental percentages of electricity prices between off-peak and peak hours

\begin{tabular}{|c||c||c|c|}
\hline \hline Year & Off-peak $(\boldsymbol{k} / \mathbf{k W h})$ & Peak $(\boldsymbol{k} / \mathbf{k W h})$ & Increment \\
\hline 2014 & 11.7 & 18.0 & $35.0 \%$ \\
\hline 2015 & 13.3 & 22.6 & $41.0 \%$ \\
\hline
\end{tabular}




\subsubsection{Using Load Shifting Strategy Planning Model during Summer (the cooling) Season}

Since in summer season the outdoor temperature is always higher than $0^{\circ} \mathrm{C}$, implementing SDFSS strategy planning model will not be advantageous. Hence, the only strategy planning model that could be implemented during summer season is LSH-SPM. In summer season, pre-cooling method should be used to cool down the zone temperature during mid-peak hours. Based on summer season TOU pricing scheme, peak hours start from 11:00 am and end at 17:00 pm. Since in summer season, pre-cooling process should be implemented during mid-peak hours, the impact of LSHSPM on energy cost saving is not as strong as it is in winter when pre-heating process takes place during off-peak hours with lower electricity prices.

The methodologies described in Section 3.2.4 are also used for pre-cooling the house in summer season. In summer season, $23.5^{\circ} \mathrm{C}$ is selected as the zone temperature set point. Based on ASHRAE Standard [4.22], during summer season zone temperature can only change between $22^{\circ} \mathrm{C}$ and $26^{\circ} \mathrm{C}$. These boundaries are set in Matlab program in order to manage and control the implementation of LSH-SPM. In other words, zone temperature is reduced to $22^{\circ} \mathrm{C}$ before peak hours start (i.e., at 10:00 am) to store cooling energy in the house and subsequently to keep the HVAC system off during peak hours.

Figures 4-2 and 4-3 show the zone temperature and operational command matrix, respectively, before implementing LSH-SPM. 


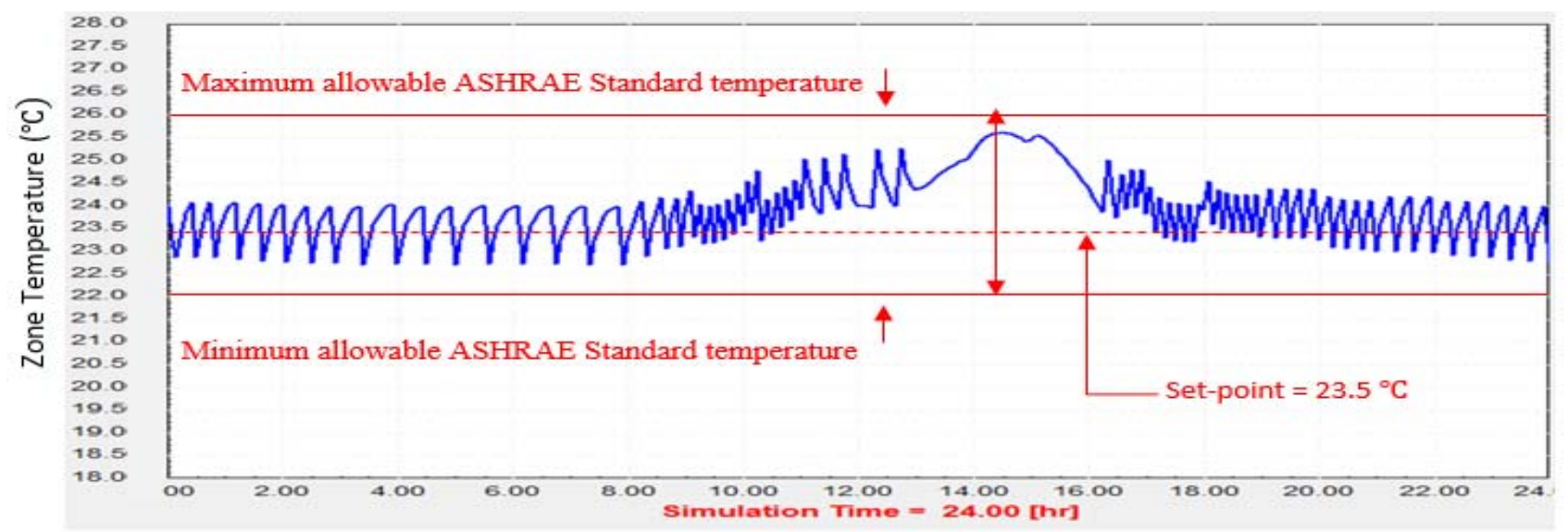

Figure 4-2: Zone temperature before implementing LSH-SPM

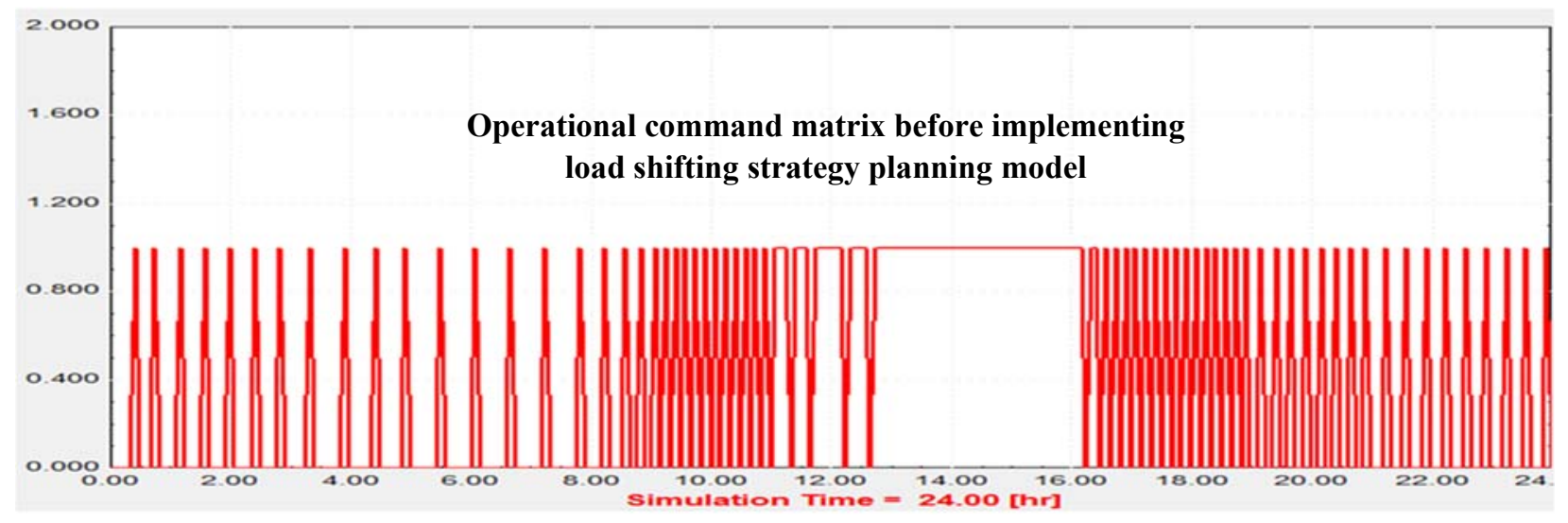

Figure 4-3: Operational command matrix before implementing LSH-SPM

Figures 4-4 and 4-5 show the zone temperature (ZT) and operational command matrix, respectively, at the first iteration of LSH-SPM. As the figure shows, first the zone temperature was reduced to $22^{\circ} \mathrm{C}$ (for pre-cooling) and then the HVAC system was kept off during the peak hours to reduce the energy cost. 


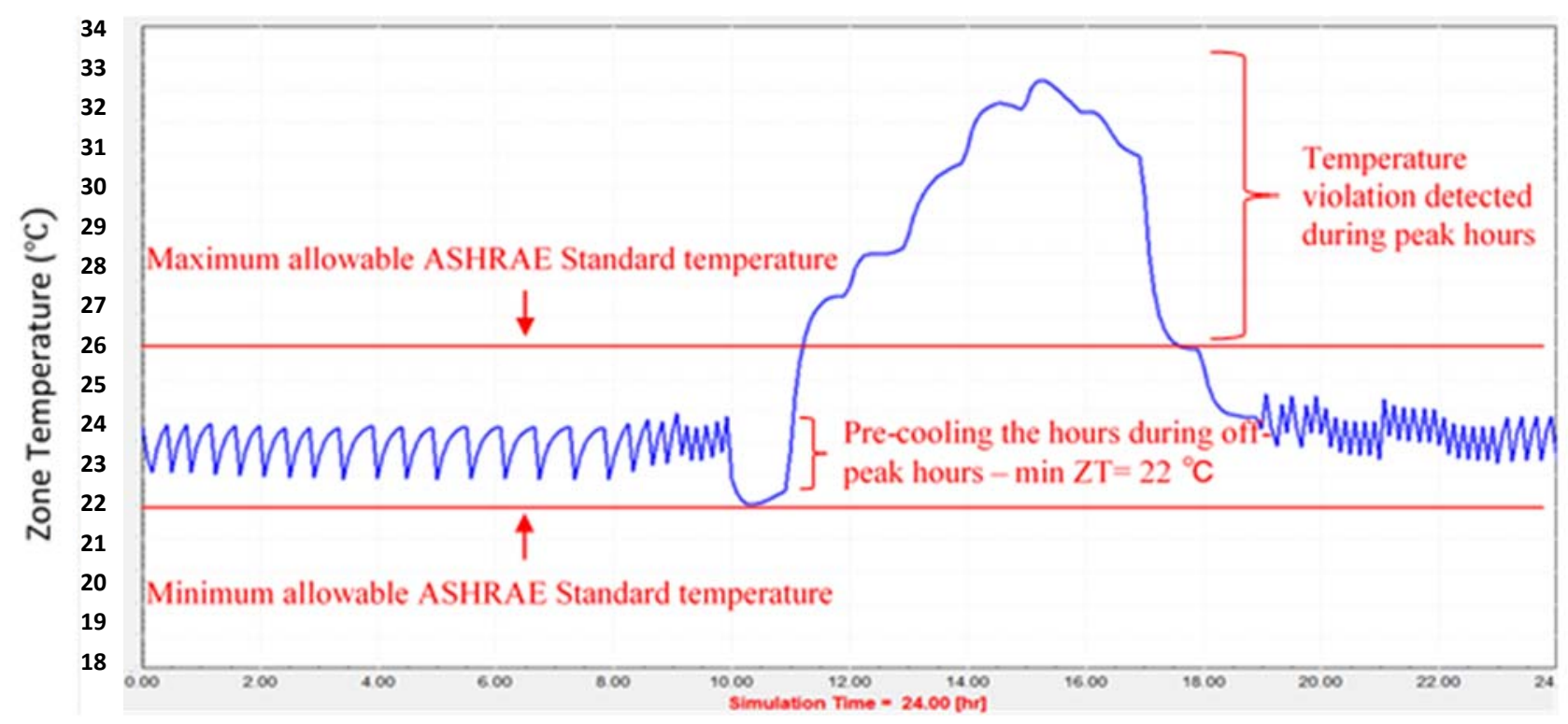

Figure 4-4: Zone temperature at the first iteration of LSH-SPM

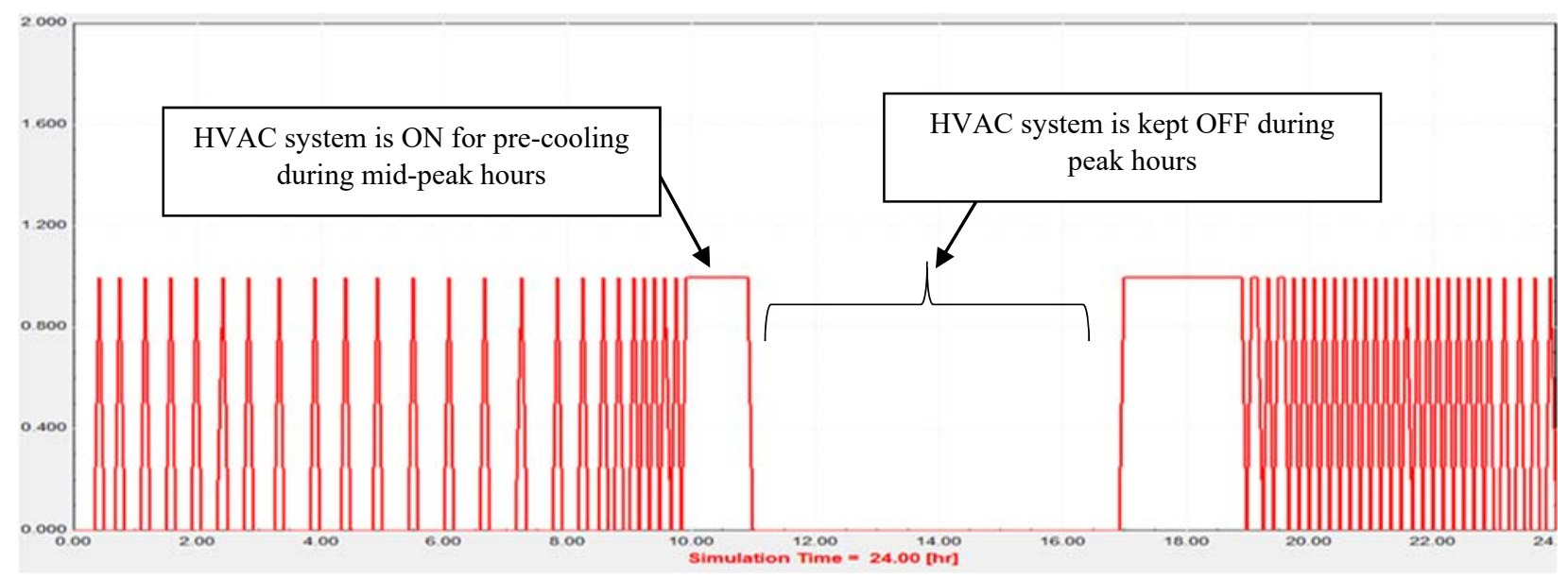

Figure 4-5: Operational command matrix at the first iteration of LSH-SPM

When the zone temperature during peak hours is higher than the upper range permitted by the ASHRAE Standard (temperature violation), the Matlab controller will turn on the HVAC system in that particular time step in the next iteration. 


\subsection{Simulation Results and Discussion}

4.3.1 Analysis of the Impact of Intelligent Strategy Planning Models on HVAC System Demand and Energy Cost During Winter (the heating) Season

\subsubsection{Heating Season Description}

A year consists of 365 days ( 8760 hours). In this chapter, the heating season was assumed to begin on October $1^{\text {st }}\left(6576\right.$ hour) and end on May $21^{\text {th }}$ (3407 hour) [4.23-4.24]. It is also considered that the indoor set-point temperature is $21^{\circ} \mathrm{C}$ during winter (the heating) season. The weather file used to simulate the thermal demand is the metropolitan Toronto weather provided in the TRNSYS library. Figure 4-6 depicts the hourly outdoor temperature during the heating season.

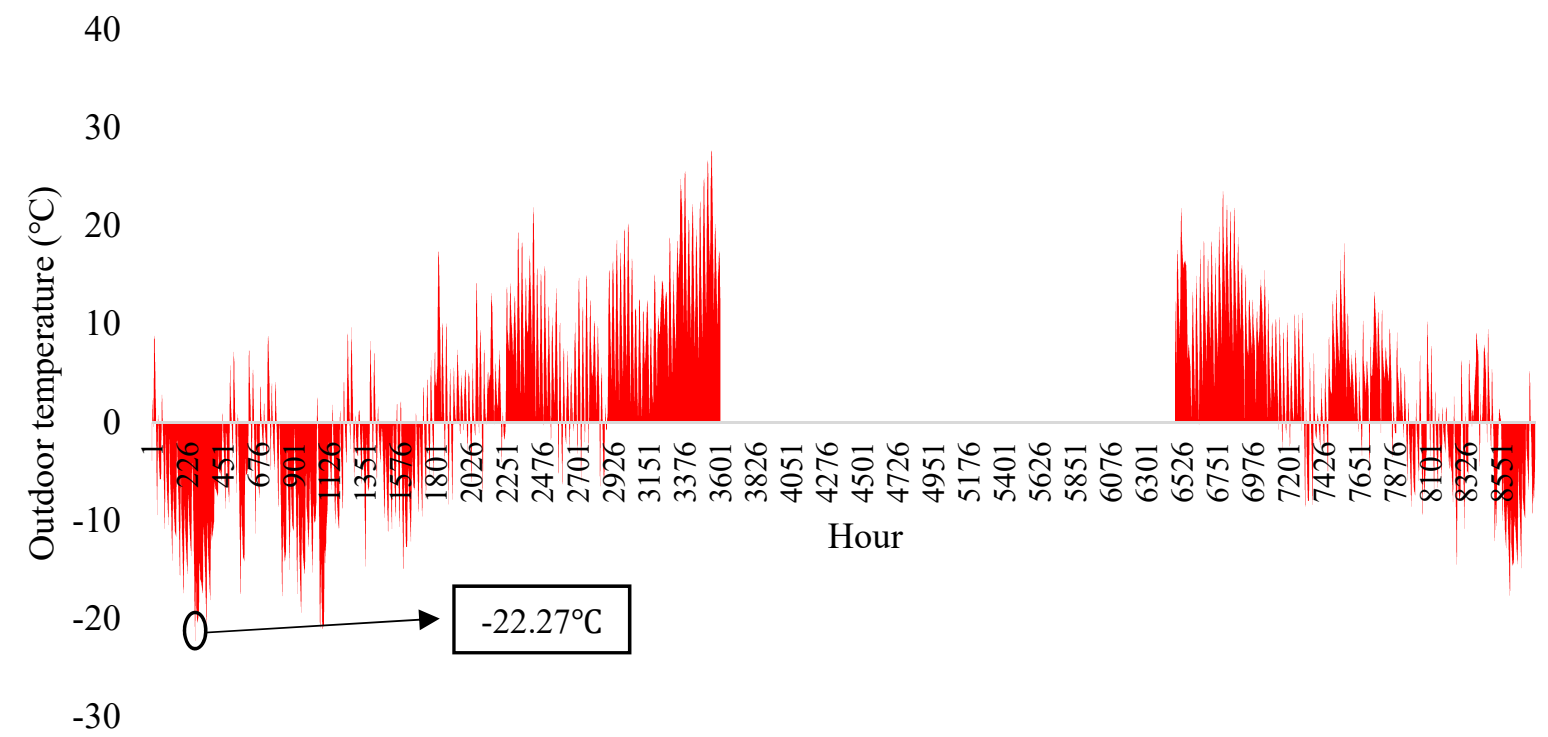

Figure 4-6: Winter (the heating) season - outdoor temperature

Based on Figure 4-6, the minimum outdoor temperature for Toronto is $-22.27^{\circ} \mathrm{C}$ and occurs on January $12^{\text {th }}$ (273 hour).

\subsubsection{Methodology}

The process system and strategy planning models described in Chapter 3 are used in this section for analyzing the behavior of HVAC system in daily basis during winter season. 2015 electricity prices presented in Section 4.2.2 are used in energy cost calculations. 


\subsubsection{Impact of Different SPMs on the HVAC System Demand and Energy Cost on Three Different Sample Days}

To consider the effects of LSH, SDFSS, and LSHSDFSS strategy planning models on the energy demand and associated cost of House A's HVAC system, three different sample days are selected to highlight the process. These sample days are December $1^{\text {st }}$, January $1^{\text {st }}$, and February $1^{\text {st }}$, i.e., the first day of each month of winter season.

Figure 4-7shows the outdoor temperature during the first sample day (December $1^{\text {st }}$ ).

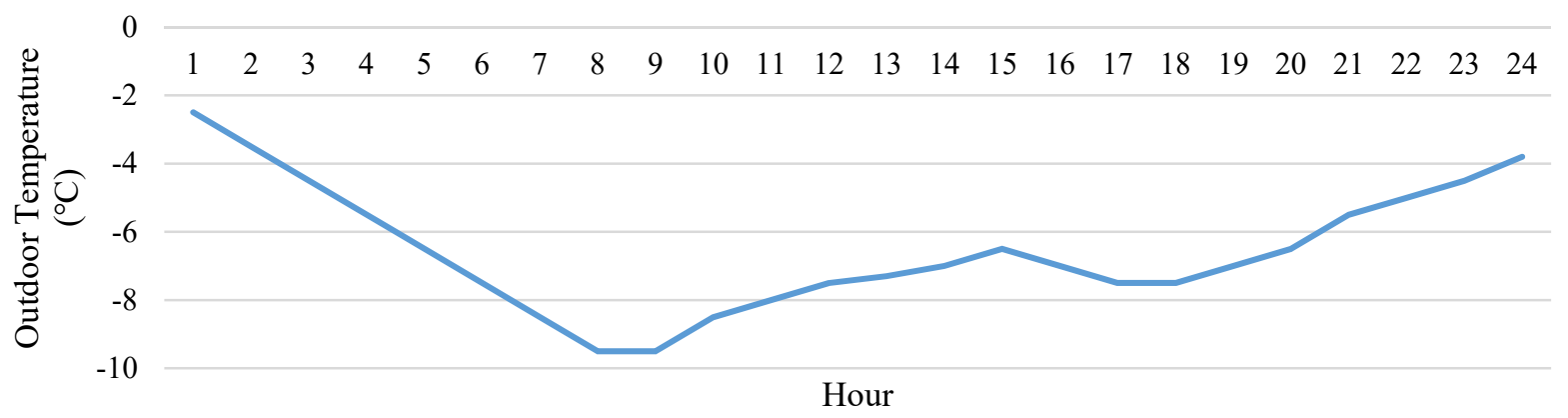

Figure 4-7: Outdoor temperature on December $1^{\text {st }}$ - TRNSYS metropolitan weather data

As Figure 4-7 shows, on December $1^{\text {st }}$, the outdoor temperature changed between $-2.27^{\circ} \mathrm{C}$ and $9.29^{\circ} \mathrm{C}$. All three SPMs were implemented on House A's HVAC system. Figure 4-8 shows the HVAC system DEC and energy cost saving based on each strategy planning model.

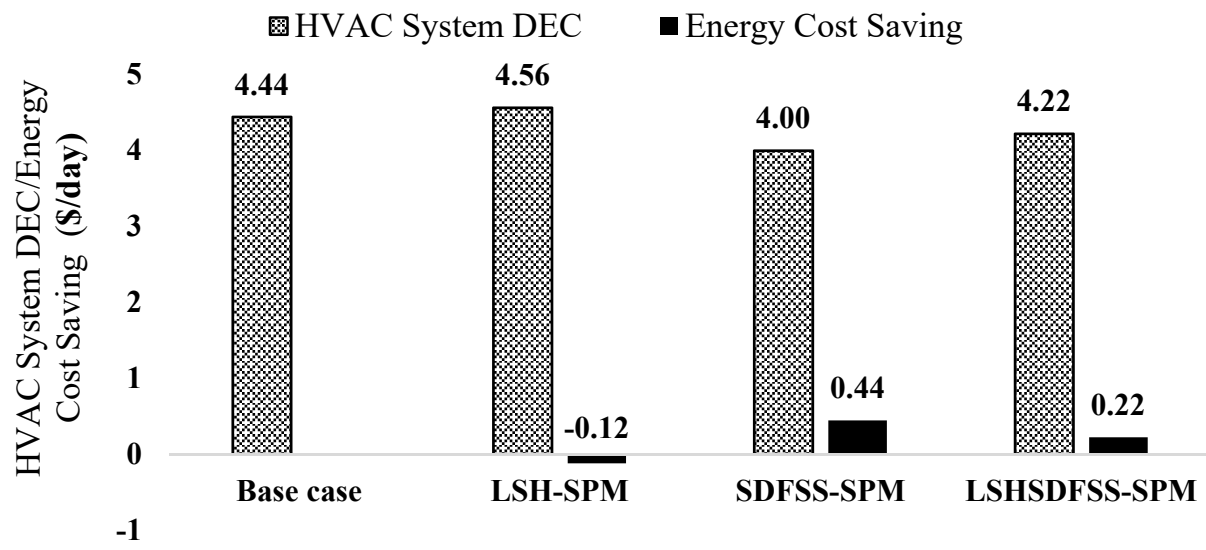

Figure 4-8: HVAC system daily energy cost (DEC) and energy cost saving based on each strategy planning model - December $1^{\text {st }}$ 
Figure 4-8 shows that on December $1^{\text {st }}$ using LSH-SPM brought no cost saving (negative energy cost saving means that the energy cost, when LSH-SPM was implemented, was higher comparing to the baseline control scenario). This was expected since on December $1^{\text {st }}$ the outdoor temperature was always lower that $0^{\circ} \mathrm{C}$. Using SDFSS and LSHSDFSS strategy planning models, $\$ 0.44$ and $\$ 0.22$ were saved, respectively. Based on the simulation results, on December $1^{\text {st }}$, the maximum amount of energy cost was reduced by using SDFSS. Figure 4-9 shows the outdoor temperature during the second sample day (January $1^{\text {st }}$ ).

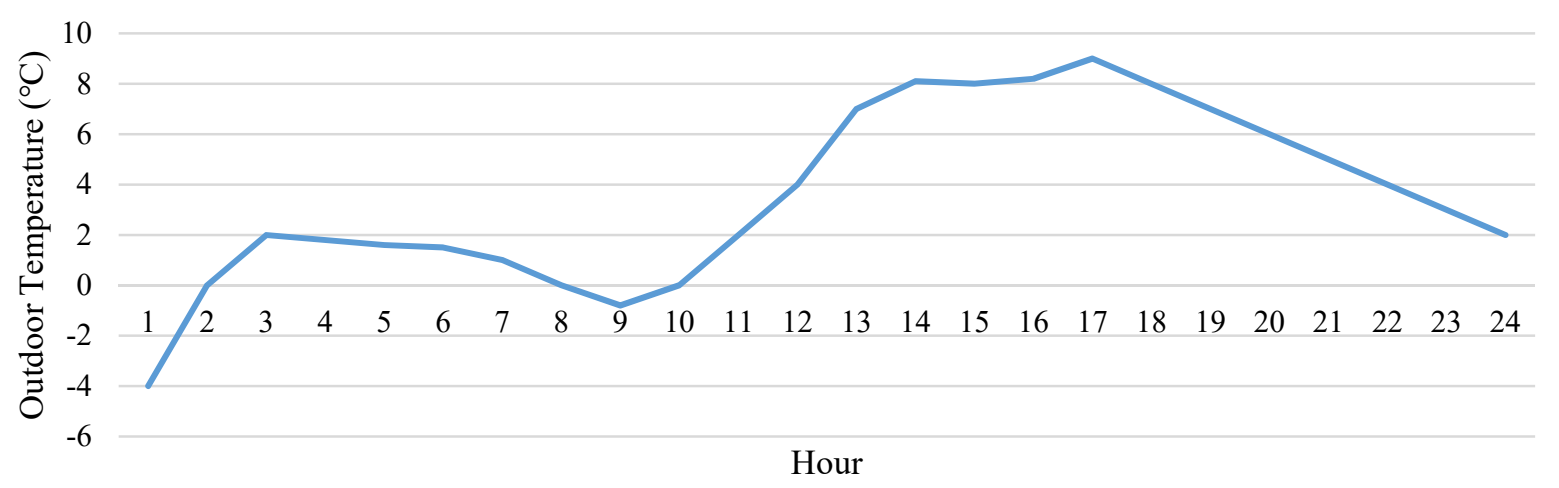

Figure 4-9: Outdoor temperature on January $1^{\text {st }}$ - TRNSYS metropolitan weather data

As Figure 4-9 shows on January $1^{\text {st }}$, the outdoor temperature changed between $-3.87^{\circ} \mathrm{C}$ and $8.25^{\circ} \mathrm{C}$. All three SPMs were implemented on House A's HVAC system on this specific day. Figure 4-10 shows the HVAC system DEC and energy cost saving based on each strategy planning model.

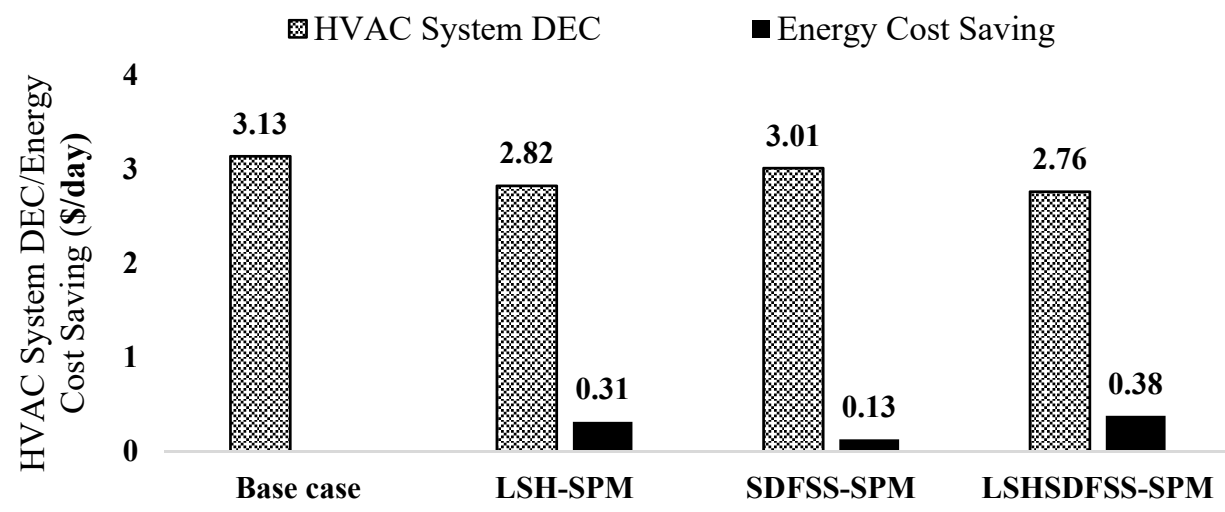

Figure 4-10: HVAC system daily energy cost (DEC) and energy cost saving based on each strategy planning model - January $1^{\text {st }}$ 
Based on Figure 4-10, LSH-SPM saved \$0.31. With SDFSS the energy cost saving was not significant since the outdoor temperature during the day was almost higher than $0^{\circ} \mathrm{C}$. Based on the results presented in Section 3.2.3.4, the HVAC system almost benefits from SDFSS-SPM at negative outdoor temperatures. Using LSHSDFSS strategy planning model saved $\$ 0.38$. Based on the simulation results, on January $1^{\text {st }}$ maximum money was saved by using LSHSDFSS-SPM. Figure 4-11 shows the outdoor temperature during the third sample day (February $1^{\text {st }}$ ).

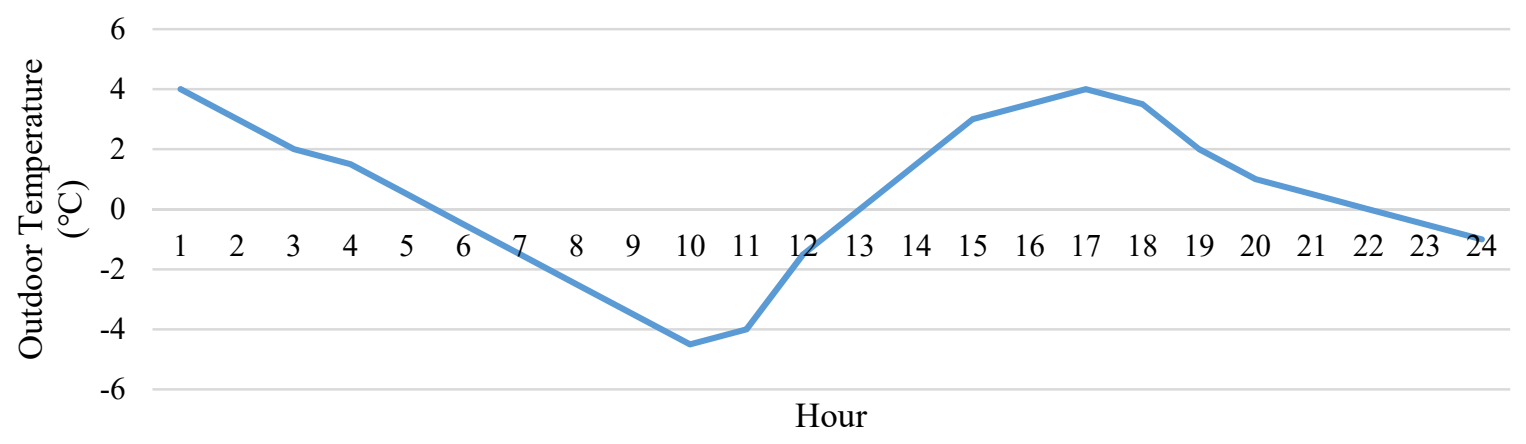

Figure 4-11: Outdoor temperature on February $1^{\text {st }}-$ TRNSYS metropolitan weather data

As Figure 4-11 shows on February $1^{\text {st }}$ the outdoor temperature changed between $-4.85^{\circ} \mathrm{C}$ and $3.97^{\circ} \mathrm{C}$. All three SPMs were implemented on House A's HVAC system on this sample day. Figure 4-12 shows the HVAC system DEC and energy cost saving based on each strategy planning model.

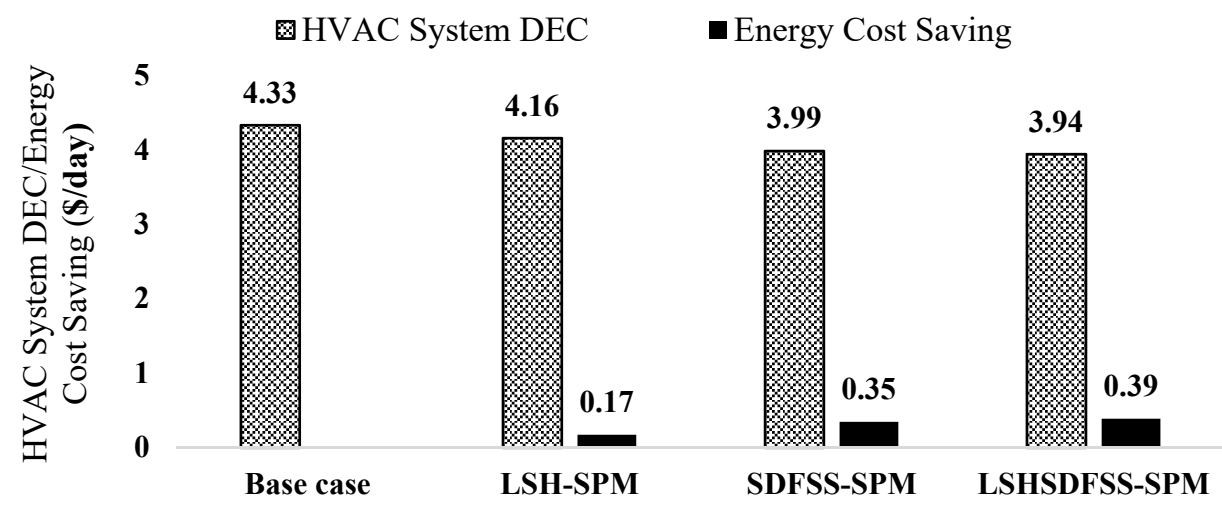

Figure 4-12: HVAC system daily energy cost (DEC) and energy cost saving based on each strategy planning model - February $1^{\text {st }}$ 
As Figure 4-12 shows, \$0.17 was saved using the LSH-SPM, \$0.35 was saved using the SDFSS, and \$0.39 was saved using the LSHSDFSS strategy planning model. Based on the simulation results, on this sample day the maximum energy cost saving was obtained by using LSHSDFSSSPM. Figure 4-13 shows the outdoor temperature at the coldest day of the year. Based on this figure, the outdoor temperature changed between $-22.27^{\circ} \mathrm{C}$ and $-11.10^{\circ} \mathrm{C}$ during this particular day.

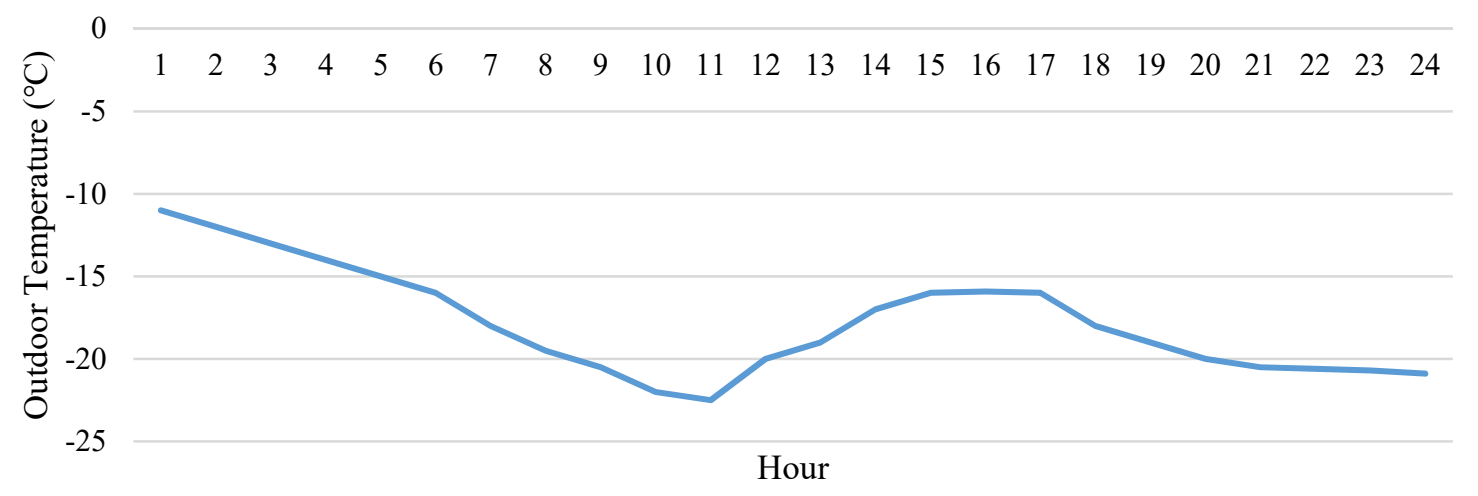

Figure 4-13: The outdoor temperature on the coldest day of year - TRNSYS metropolitan weather data

All three SPMs were implemented on House A's HVAC system on February $1^{\text {st }}$. Figure 4-14 depicts the HVAC system DEC and energy cost saving based on each strategy planning model.

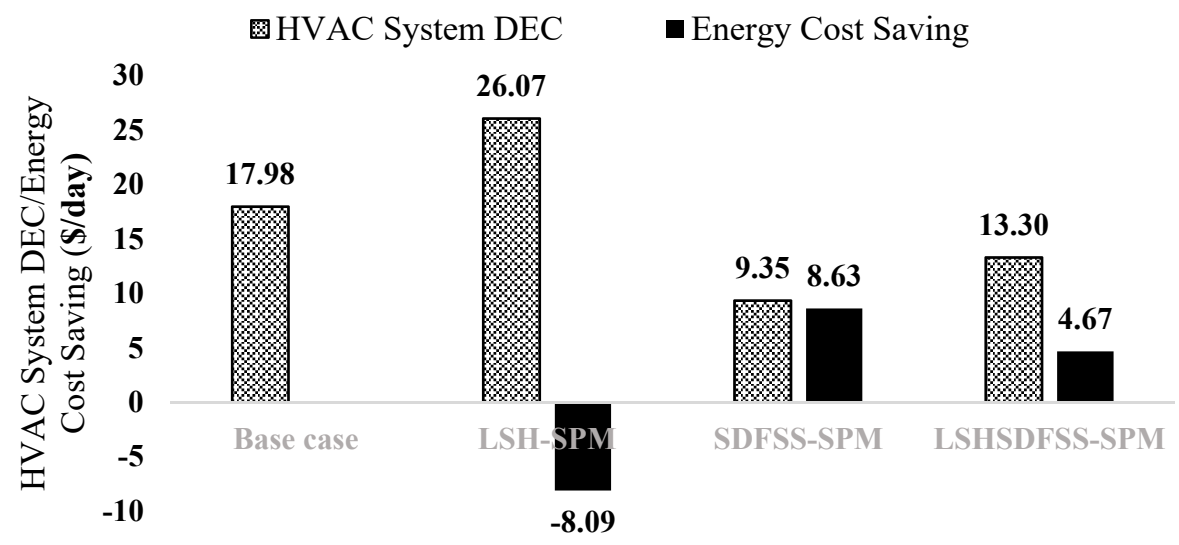

Figure 4-14: HVAC system daily energy cost (DEC) and energy cost saving based on each strategy planning model on the coldest day of the year 
As the figure shows no saving was achieved using LSH-SPM due to the extremely cold weather condition. The energy cost saving using SDFSS was \$8.63. In other words, there was $48 \%$ reduction on daily energy cost of HVAC system when SDFSS-SPM was implemented in this extremely cold weather condition. $\$ 4.67$ is saved using LSHSDFSS strategy planning model. Based on the simulation results, on February $1^{\text {st }}$ the maximum saving happened when SDFSSSPM is utilized.

\subsubsection{Energy Cost Saving Analysis by Utilizing Different SPMs in the Heating Season}

In this section, the influence of each strategy planning model in the heating season is analyzed. Figure 4-15 depicts the DEC of HVAC system with different strategy planning models. In this figure, simulation starts on January $1^{\text {st }}$ and ends on December $31^{\text {th }}$, containing 233 days (the cooling season days are excluded). The red line (the first line in the legend) shows the energy cost of HVAC system when no strategy planning model is implemented on the system (baseline control scenario). The second line (the green line) shows the energy cost of HVAC system with LSHSDFSS-SPM. The third line (the blue line) shows the energy cost of HVAC system with LSHSPM. The last line (the black line) shows the energy cost of HVAC system with SDFSS-SPM.

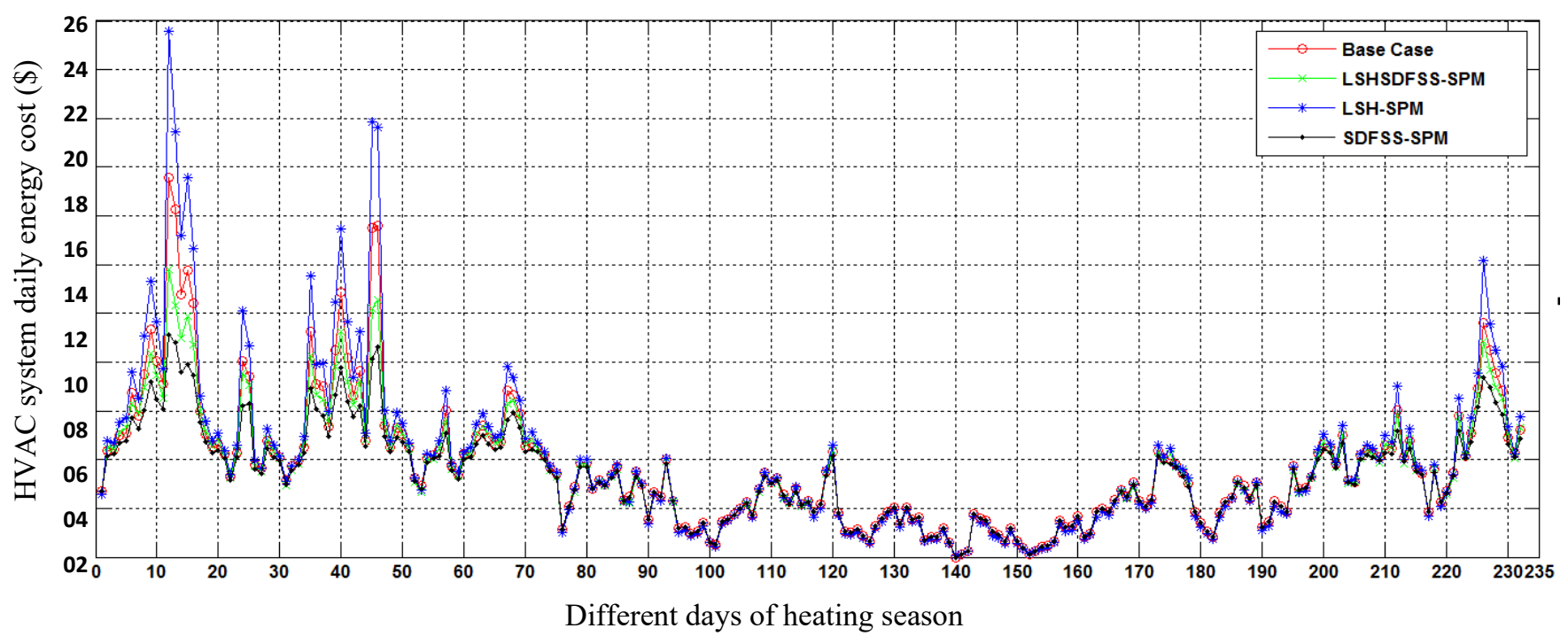

Figure 4-15: DEC of HVAC system based on different strategy planning models in the heating season 
As it can be concluded from the Figure 4-15, when the outdoor temperature is cold and/or very cold (i.e., during winter months), the DEC of HVAC system increased using the LSH-SPM. However, during this period, the DEC of HVAC system decreased significantly when using SDFSS-SPM. LSHSDFSS-SPM took the second place in reducing the DEC of HVAC system during these cold days. When the outdoor temperature is not very cold (i.e., at the beginning and/or the end of the heating season - the middle section of the graph in Figure 4-15) there was no noticeable difference in energy cost saving using different SPMs.

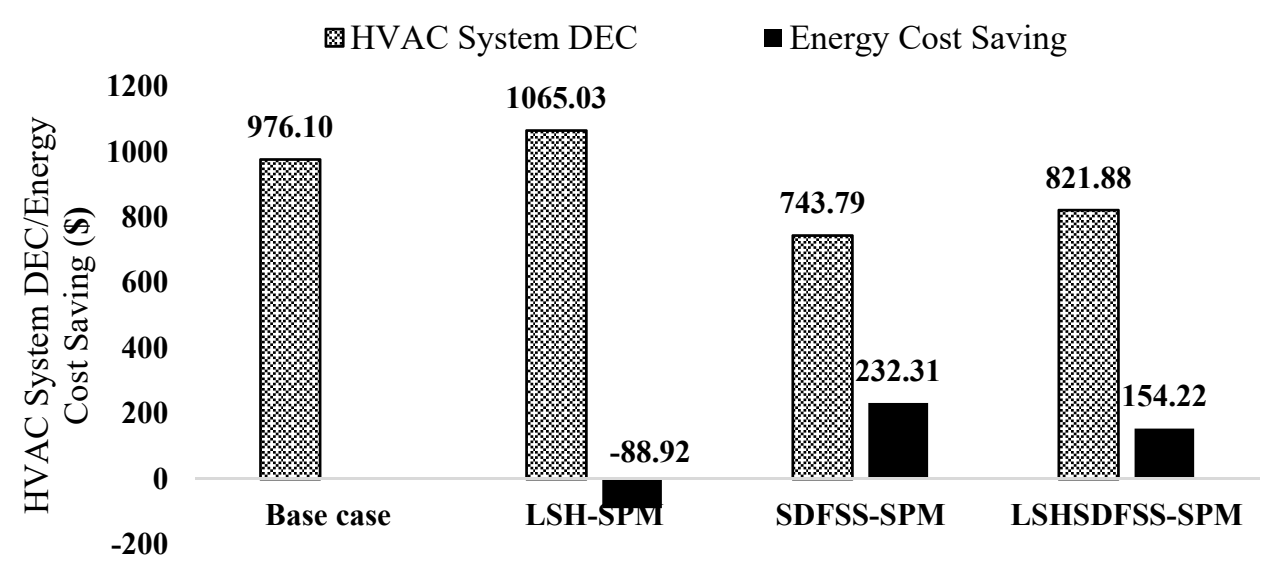

Figure 4-16: Energy cost saving with different strategy planning models in the heating season

Figure 4-16 illustrates the energy cost saving with different strategy planning models for the heating season. The total energy cost for the baseline scenario is $\$ 976.10$. The HVAC operating cost increased to $\$ 1065.03$ using LSH-SPM. In other words, despite the fact that LSH-SPM has the potential to reduce the operating cost of HVAC system (when the daily average outdoor temperature changes between $0^{\circ} \mathrm{C}$ and $12^{\circ} \mathrm{C}$ ), the HVAC system operating cost increased by a total of $9.11 \%$ when this strategy planning model was implemented for the entire heating season. The operating cost of HVAC system decreased significantly with implementation of SDFSS-SPM in the heating season. This fuel switching strategy planning model reduced the HVAC system operation cost by $23.8 \%$.

The last strategy planning model implemented on the system was LSHSDFSS which reduced the HVAC system operating cost by $15.8 \%$. 


\subsubsection{Optimal Strategy Planning Model}

As described before, there is a strong relationship between the effectiveness of each strategy planning model and the weather condition, particularly the outdoor temperature. As a result, on cold (or extremely cold) weather condition, the optimal strategy planning model for minimizing the HVAC system operating cost is SDFSS-SPM. When daily average outdoor temperature changes between $0^{\circ} \mathrm{C}$ and $12^{\circ} \mathrm{C}$, LSH-SPM can be selected as the optimum strategy planning model. LSHSDFSS-SPM takes advantage of both load shifting and fuel switching. This strategy planning model can be used as the optimum SPM on certain days when the daily average outdoor temperature changes between $0^{\circ} \mathrm{C}$ and $8^{\circ} \mathrm{C}$. Considering these specifications, an optimization process is executed to determine the best SPM, i.e., the one that generates the minimum HVAC system daily energy cost, on each specific day.

Figure 4-17 depicts the minimum daily operating cost of HVAC system after running optimization process for the heating season. The overall operating cost of HVAC system in the heating season after utilizing the optimization process is $\$ 716.08$. This optimum/minimum cost is lower than the HVAC system operating cost of $\$ 743.79$ with SDFSS-SPM (as the most effective SPM during the heating season) because on some winter days, LSH or LSHSDFSS SPM generated lower energy cost for HVAC system operation due to the specific weather conditions. The implementation of the fully optimized HVAC SPM achieves a $26.6 \%$ energy cost reduction compared to the base case control for a typical Toronto heating season.

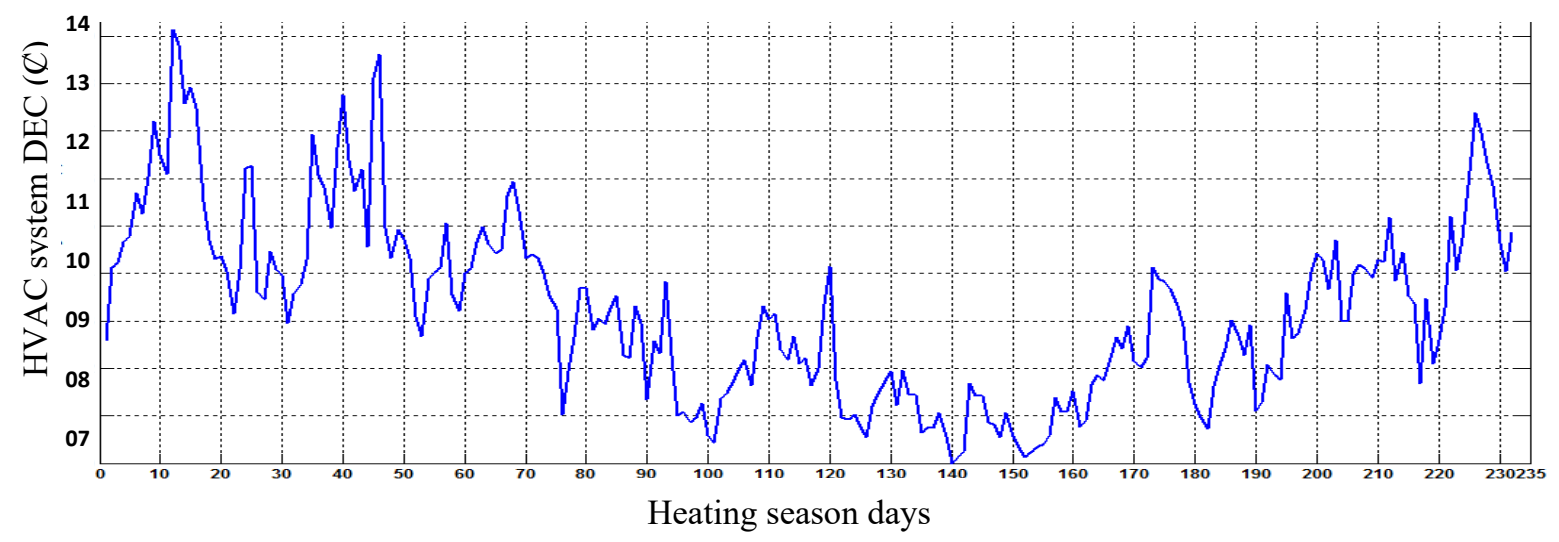

Figure 4-17: Minimum daily operating cost of HVAC system - heating season days 


\subsubsection{Impact of the Load Shifting Strategy Planning Model on HVAC System During Summer (the Cooling Season)}

\subsubsection{Cooling Season Period}

In this chapter, the cooling season was assumed to begin on May $22^{\text {th }}$ and end on September $30^{\text {th }}$ [4.24]. Zone set-point temperature is set to $23.5^{\circ} \mathrm{C}$ during summer (the cooling) season. The metropolitan Toronto weather file available at the TRNSYS library was used to simulate the thermal demand. Figure 4-18 depicts the hourly outdoor temperature during the cooling season. The maximum outdoor temperature was $33.96^{\circ} \mathrm{C}$ and was recorded on July $23^{\text {th }}$ (1433 hour).

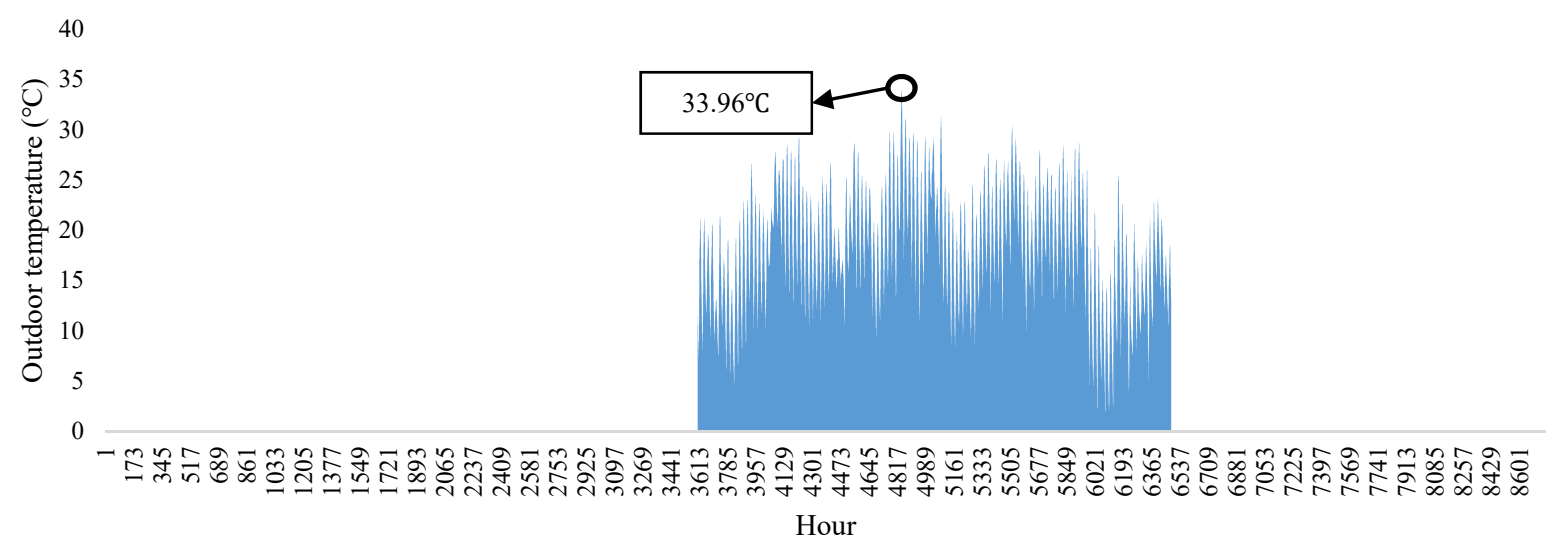

Figure 4-18: Summer (cooling) season - outdoor temperature

\subsubsection{Methodology}

The process system and LSH strategy planning model described in Section 4.2.3 are used in this section for analyzing the behavior of HVAC system in the cooling season.

\subsubsection{Impact of the LSH-SPM on HVAC System Demand and Energy Cost in the Cooling Season}

Since outdoor temperature during the cooling season is always above $0^{\circ} \mathrm{C}$, SDFSS and consequently LSHSDFSS strategy planning models are not advantageous. Hence, only LSH-SPM is implemented in the cooling season. To this end, pre-cooling method is used for cooling down the zone temperature during mid-peak hours. Hence, the impact of LSH-SPM on HVAC system energy demand and cost saving in summer is not as strong as it is in winter season in which preheating process takes place during off-peak hours with lower electricity cost. 


\subsubsection{Energy Cost Saving Analysis by Utilizing LSH-SPM in the Cooling Season}

In this section, the influence of LSH-SPM is analyzed in the cooling season. Figure 4-19 depicts the DEC of HVAC system after implementing LSH strategy planning model. As mentioned in Section 4.3.2.1, the cooling season starts on May $22^{\text {th }}$ and ends on September $30^{\text {th }}$, containing 132 days. In Figure 4-19, the first line (the red line) shows the energy cost of HVAC system when no strategy planning model is implemented (baseline control scenario). The second line (the blue line) shows the HVAC system energy cost with LSH-SPM.

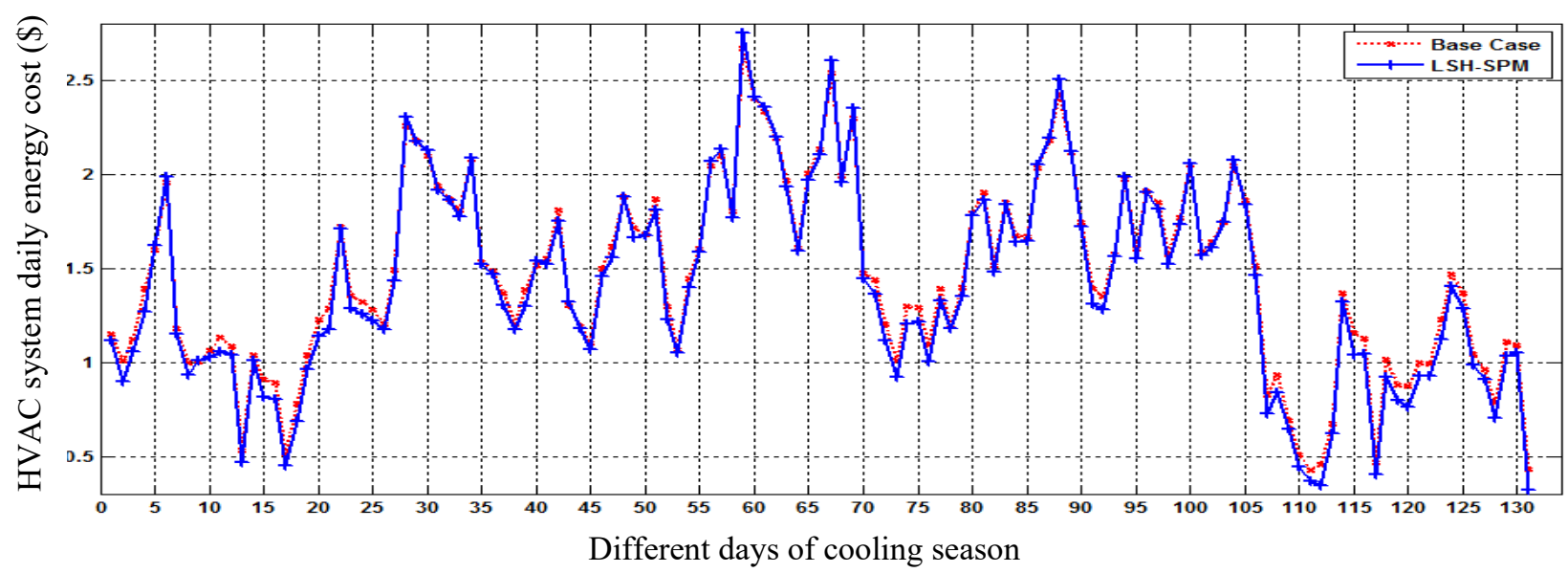

Figure 4-19: DEC of HVAC system before and after taking advantage of LSH-SPM in the cooling season

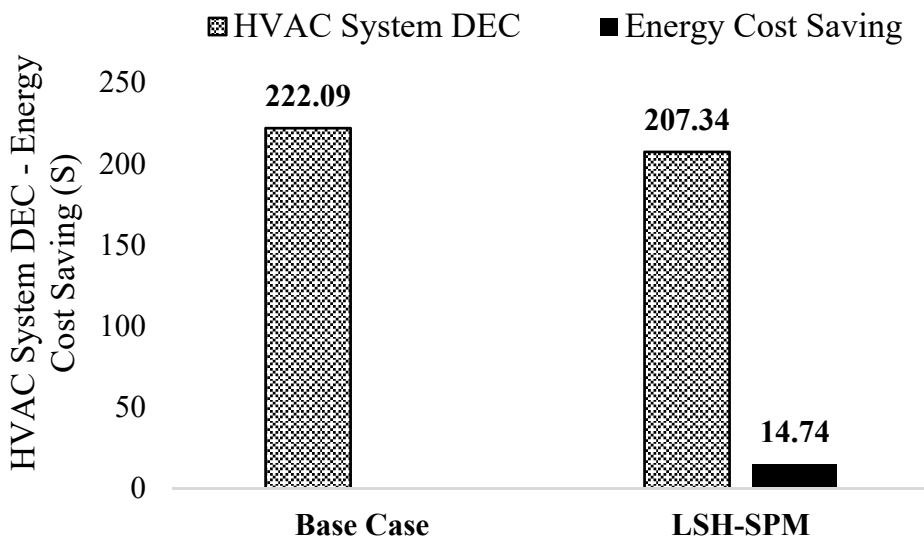

Figure 4-20: Energy cost saving implementing LSH strategy planning model in the cooling season 
Figure 4-20 illustrates the cost saving in the cooling season when LSH strategy planning model is implemented. As the figure shows, the total energy cost of HVAC system operation during the cooling season is \$222.09 in the baseline control scenario. Using LSH-SPM the HVAC operating cost decreased to $\$ 207.34$. In other words, LSH strategy planning model reduced the HVAC system operating cost by $6.63 \%$ for the cooling season. As mentioned before, since in summer season pre-cooling process should be executed during mid-peak hours, the daily impact of LSHSPM on energy cost saving is not as effective as in winter season when pre-heating process takes place during off-peak hours with lower electricity prices.

\subsection{Conclusion}

Residential houses/buildings can play significant role in managing the network energy system. Since residential houses/buildings have the potential of storing thermal (heat or cold) energy, they present great opportunity for managing/controlling HVAC system energy demand/cost by utilizing controllers that take advantage of smart strategy planning models. This chapter investigated the effectiveness of three novel strategy planning models on the HVAC system energy cost in winter and summer seasons. SDFSS-SPM was the first strategy planning model applied for selecting the least expensive hot-air supplier at each hour. This SPM could decrease home owners' HVAC energy cost significantly. When the outdoor temperature was below $8^{\circ} \mathrm{C}, \mathrm{DFC}$ decreased using this model. As the outdoor temperature got colder, the savings increased. The second strategy planning model used in this chapter was LSH-SPM model. This model shifted the HVAC load from peak to off- and/or mid-peak hours. Since the outdoor temperature and house characteristics directly affect the thermal energy to be stored inside a house, a smart method was used to determine the best starting time for pre-heating/cooling the house. This method not only ensures the thermal comfort level, but also minimizes the ASHP energy demand and cost. Based on simulation results, when the daily average outdoor temperature changed between $0^{\circ} \mathrm{C}$ and $12^{\circ} \mathrm{C}$, the best results were obtained by implementing LSH-SPM model. LSHSDFSS-SPM was the third strategy planning model investigated. This SPM was developed by integrating SDFSS-SPM and LSH-SPM models together into one. LSHSDFSS-SPM takes advantage of load shifting and fuel switching system simultaneously. This SPM was most effective when the daily average outdoor temperature changed between $0^{\circ} \mathrm{C}$ and $8^{\circ} \mathrm{C}$. The aforementioned SPMs were implemented on TRCA ASH 
House A's HVAC system during the heating and cooling seasons. In the heating season, the operating cost of the HVAC system decreased significantly at $23.8 \%$ by implementing SDFSSSPM. LSHSDFSS-SPM reduced the HVAC system operating cost by $15.8 \%$. Despite the fact that LSH-SPM can reduce the operating cost of an HVAC system, the HVAC system operating cost was totally increased by $9.11 \%$ by implementing this strategy planning model during the heating season. The case study house's low thermal mass as well as cold/very cold outdoor temperature in Toronto (Canada) can be considered as the most important parameters that decreased the effectiveness of LSH-SPM for the winter season. In the cooling season, LSH strategy planning model was the only SPM implemented and it reduced the HVAC system operating cost by $6.63 \%$.

\subsection{References:}

4.1. Xu, X., Taylor, J., Pisello, A., "Network synergy effect: Establishing a synergy between building network and peer network energy conservation effects", Energy and Buildings, 2014. 68: p. 312-320.

4.2. Xu, X., Taylor, J., Pisello, A., Culliga, P., "The impact of place-based affiliation networks on energy conservation: An holistic model that integrates the influence of buildings, residents and the neighborhood context", Energy and Buildings, 2012. 55: p. 637-646.

4.3. Pagani, G., Aiello, M., "The Power Grid as a complex network: A survey”, The Electricity Journal, 2013. 26(6): p. 74-78.

4.4. Siano, P., Sarno, D., "Assessing the benefits of residential demand response in a real time distribution energy market", Applied Energy, 2016. 161: p. 533-551.

4.5. Arteconi A., Ciarrocchi, E., Pan, Q., Carducci, F., "Thermal energy storage coupled with PV panels for demand side management of industrial building cooling loads", Applied Energy (2016)

4.6. Vakiloroaya, V., Somali, B., Fakhar, A., Pishghadam, K., “A review of different strategies for HVAC energy saving”, Energy Conversion and Management, 2014. 77: P. 738-754.

4.7. Huang, H., Chen, L., Hu, E., "A new model predictive control scheme for energy and cost savings in commercial buildings: An airport terminal building case study", Building and Environment, 2015. 89: P. 203-216.

4.8. Ghahramani A., Zhang, K., Dutta, K., Yang, Z., Gerber, B., "Energy savings from temperature setpoints and deadband: Quantifying the influence of building and system properties on savings", Applied Energy, 2016. 165: P. 930-942.

4.9. Kim, W., Jeon, Y., Kim, Y., "Simulation-based optimization of an integrated daylighting and HVAC system using the design of experiments method", Applied Energy, 2016. 162: P. $666-674$. 
4.10. Nazi, W., Wang, Y., Roskilly, T., "Methodologies to Reduce Cooling Load using Heat Balance Analysis: A Case Study in an Office Building in a Tropical Country", Energy Procedia, 2015. 75: P.1269 - 1274.

4.11. Christantonia, D., Flynna, D., Finnb, D., "Modelling of a Multi-purpose Commercial Building for Demand Response Analysis", Energy Procedia, 2015. 78: P. 2166 - 2171.

4.12. Kramer, R., Maas, M., Martens, M., Schijndel, A., Schellen, H., "Energy conservation in museums using different setpoint strategies: A case study for a state-of-the-art museum using building simulations", Applied Energy, 2015. 158: P. 446-458.

4.13. Wang, Q., Augenbroe,G.,Kim, J., Gu, L., "Meta-modeling of occupancy variables and analysis of their impact on energy outcomes of office buildings", Applied Energy, 2016. 174: P. $166-180$

4.14. Patteeuw, D., Gregor, P., Helsen, L., "Comparison of load shifting incentives for low-energy buildings with heat pumps to attain grid flexibility benefits", Applied Energy, 2016. 167: P. 80-92.

4.15. Beizaee, A, Allison, D., Lomas, K., Foda, E., Loveday, D., "Measuring the potential of zonal space heating controls to reduce energy use in UK homes: The case of un-furbished 1930s dwellings", Energy and Buildings, 2015. 92: p 29-44.

4.16. Chassin, DP., Stoustrup, J., Agothoklis, P., Djilali, N., "A new thermostat for real-time price demand response: Cost, comfort and energy impacts of discrete-time control without deadband", Applied Energy, 2015. 155: p. 816-825.

4.17. Li, J., Poulton, G., Platt, G., Wall, j., James, G., "Dynamic zone modelling for HVAC system control", Int J of Model, Identif and Control, 2010. 9(1/2): p. 5-13.

4.18. Radhakrishnan, N., Su, Y., Su, R., Poolla, K., "Token based scheduling for energy management in building HVAC systems", Applied Energy, 2016. 173: P. 67-79.

4.19. Griful, S., Jacobsen, R., Nguyen, D., Sorensen, G., "Demand response potential of ventilation systems in residential buildings", Energy and Buildings, 2016. 121: P. 1-10.

4.20. Di Giorgio, A., Liberati, F., "Near real time load shifting control for residential electricity prosumers under designed and market indexed pricing models", Applied Energy, 2014. 128: p. 119-132.

4.21. Kamel, R., Fung, A.S., "Modeling, simulation and feasibility analysis of residential BIPV/T+ASHP system in cold climate-Canada", Energy and Buildings, 2014. 82: p. 758770.

4.22. ASHRAE 2009, ASHRAE Handbook-Fundamentals, American Society of heating, Refrigeration and Air-Conditioning Engineers, Inc., Atlanta, GA

4.23. Zhang, D., Barua, R., Fung, A., "TRCA-BILD Archetype Sustainable House - Overview of Monitoring System and Preliminary Results for Mechanical Systems, ASHRAE Transactions, 2011: p.597-612.

4.24. Safa, A., Fung, A., Kumar, R., "Performance of two-stage variable capacity air source heat pump: Field performance results and TRNSYS simulation”, Energy and Buildings, 2015. 94: p. 80-90. 


\section{Chapter 5: Development of the Second MPC Controller to Determine Optimal HVAC Set Point Profile Using On-site Renewable Energy Generation and Storage}

The development of second MPC controller is discussed in this chapter. This chapter covers the contents of the third journal paper that has been submitted to the Journal of Applied Energy. In the second MPC controller, the simplified process/plant models have been used for THE house thermal energy and HVAC systems. The proposed MPC model was developed with the objective of reducing residential house heating, ventilating, and air conditioning (HVAC) systems energy demand and energy cost while maximizing the use of on-site renewable energies. The prominent effects of outdoor disturbances, control time horizon (TH), and HVAC operating time step (TS) were considered into the model design. Utilizing exhaustive search method and linear optimization schemes, the MPC model is capable of finding the potential optimum results. Using an intelligent algorithm including smart decision support systems significantly reduced the model processing/optimization time. Considering the cost of different energy sources, including time of use pricing scheme (TOU), operating, and capital costs of renewable sources/battery bank storage system as the weighting factors, presented the opportunity for the novel MPC model to find the optimum TH, TS, and the corresponding optimum indoor set point profile. Simulation results showed that the developed MPC controller offered significant savings on the HVAC system energy demand and associated cost without compromising the thermal comfort when compared to the base case scenarios (conventional on-off controllers) and a Simple Rule-based (SRB) controller as an efficient HVAC controller.

\subsection{Introduction}

To date, the residential sector accounts for a major energy customer [5.1, 5.2] consuming more than $40 \%$ of the entire world's energy and producing $33 \%$ of the world's carbon dioxide emissions [5.2]. Most of the residential energy consumptions are for heating, ventilation, and air conditioning (HVAC) systems. Despite their significance, the current HVAC systems do not operate in the most efficient ways under the effects of existing controllers in the residential house/building. As a result, using advanced controllers such as model-based predictive controllers (MPCs), for managing the HVAC system operation, has never been more vital. This chapter discusses the development of a 
novel model-based predictive control (MPC) scheme that manages HVAC system operation while taking advantage of different optimization schemes and smart decision support systems.

Currently, residential HVAC systems are mainly controlled by simple on/off or proportionalintegral-derivative (PID) systems as the conventional controllers [5.3]. Due to the dynamic internal/external disturbances and the time delay in house thermal energy systems, these controllers are unable to keep the zone temperature within the desired band, increasing the HVAC system demand and energy cost. To address the weakness of conventional on/off and PID controllers, different types of MPCs have been previously developed. MPC provides an anticipatory control mechanism by predicting the future state of the system based on the estimate of components' behavior and produces a constrained control matrix, which drives the system towards the optimum state [5.4]. MPC finds the optimal solution for different optimization problems by using a cost function (i.e., energy consumption). Based on the result of the previous surveys [5.5, 5.6, 5.7], higher energy and cost saving could be obtained by using a properly designed MPC controller. Among the developed MPCs for residential houses, the controllers that focus on the HVAC systems offer more energy cost saving opportunities [5.4]. For example, Ma et al. [5.7] used an MPC controller to control the zone temperature. The result of this research shows considerable saving (28\%) compared to the conventional controllers. However, since EnergyPlus is used for modeling the building and its HVAC systems, running the model results in a significant computational time. Prívara and colleagues [5.8] described an MPC model to control the room temperature. MPC used 29\% less energy than a finely-tuned controller.

Moroşan et al. [5.9] precisely managed zone temperature during occupancy period by utilizing centralized and distributed MPC controllers. In comparison with PI controller, a 36.7\% increase in thermal comfort was achieved by using these MPC controllers. Huang et al. [5.10] designed a hybrid model predictive control combining a classical MPC with a neural network feedback linearization method to reduce the HVAC system energy cost. Hilliarda et al. [5.11] developed an MPC model for a LEED Silver-certified building. The simulation results indicated a 10\% HVAC energy reduction. Deterministic and stochastic model predictive control concepts have been developed and applied to a domestic micro-grid system [5.12]. These control concepts optimized the deployment of renewable energy sources. Razmara et al. [5.13] developed an exergy model for evaluating the efficiency of HVAC systems using MPC technique. This exergy-based MPC model reduced exergy destruction by $4 \%$ and saved $12 \%$ energy demand. In another study by Lee et al. 
[5.14], on-site energy generation and storage systems were combined with an MPC framework to optimally determine the control profiles of an HVAC system for managing the demand response. Ascionea et al. [5.15] optimized building energy performance and thermal comfort using simulation-based model predictive control. The designed MPC model generated up to 56\% reduction in HVAC operating cost. Mirakhorli and Dong [5.16] provide a comprehensive review of research on traditional and advanced MPC controllers with a focus on occupancy-based model predictive control. In this paper, different physics-based and data driven models have been reviewed for modeling control-oriented buildings. Three different strategy planning models for controlling heat pumps are presented in an MPC model in [5.17]. These strategists could achieve up to $30 \%$ saving in heat pumps operating cost while increasing the degree of energy selfconsumption of heat pumps by $12 \%$. The results of two real world trials of an MPC controller in a commercial building HVAC system have been presented by West et al. [5.18]. Using the developed MPC model for a long 51 days and a short 10 days periods brought an average of $32 \%$ and 19\% energy reduction, respectively. Dobbs and Hencey [5.19] developed an occupancypredicting control algorithm for a residential HVAC system using an MPC strategy. The algorithm's effectiveness has been validated by real world occupancy data. The theory and application of different MPC controllers employed on HVAC systems have been reviewed by Afram and Janabi-Sharifi [5.20]. The authors also present an overview of the future direction of MPC controllers. Zhang et al. [5.21] developed a coordinated operation framework in an MPC model for a grid-connected residential house. This framework demonstrated economic and flexible behaviors comparing traditional control methods. Liang et al. [5.22] discuss the development of a system-level control design and parametric modeling approach to enhance the energy efficiency of an HVAC system using MPC framework. Maasoumy et al. [5.23] have designed a parameteradaptive building (PAB) and robust model predictive control (RMPC) models to handle uncertainty in an MPC platform and compared the effectiveness of these two models with that of nominal MPC and RBC platforms. Afram and Janabi-Sharifi [5.24] developed gray box models for a real residential archetype house HVAC system. The developed framework can be used to investigate the effectiveness of different control strategies like MPC and PID. A min-max optimization problem has been converted to a linear program in [5.25] to establish an economic model predictive control (EMPC) method. The developed method demonstrated a significant capability in shifting peak load, reducing HVAC system energy cost. In [5.26], the effects of 
mixed-mode cooling system and differences in occupant's thermal comfort preference have been modeled in an MPC platform. Simulation results proved the capability of the model in reducing HVAC energy consumption and satisfying the occupant's thermal comfort preferences. The benefits of using occupant's thermal comfort feedback have been examined in [5.27]. To this end, a novel dynamic thermal sensation (DTS) model has been integrated into an MPC platform. Significant energy saving was achieved using the developed platform. Kavgic et al. [5.28] identified building characteristics that have positive impact on performance of MPC controllers. Furthermore, they identified prototypical commercial and institutional buildings categories that are most suitable for MPC applications. Zakula et al. [5.29] designed a modeling environment as a simulation tool for building and employing MPC controllers. This modeling environment prepares a testbed for investigating the effectiveness of different MPC schemes.

Despite significant efforts have been previously dedicated into the development of MPC controllers, they still suffer from unacceptably long computational (optimization) time. MPC models examine many different possible scenarios within the defined process space to find the optimum state of the system; hence, they have unacceptably long optimization time. To mitigate computational time problem, this chapter discusses the development of a novel MPC controller called Demand MPC controller. In this novel MPC controller, instead of considering all existing scenarios, an intelligent algorithm/mechanism has been developed that empowers the MPC controller to detect and extract the most useful scenarios within the defined process space.

The developed intelligent algorithm takes advantage of novel smart decision support systems to detect the most useful indoor set point profiles (which result in reducing the HVAC system demand and energy cost) considering on-site renewable energy generation, energy storage, dynamic outdoor disturbances and time of use (TOU) energy prices. The optimum indoor set point profile that leads to minimum HVAC system energy cost is then selected from the useful set point profiles using linear optimization. The reduced computational time, which occurred by taking the advantage of intelligent algorithm, allows the novel MPC controller to examine different control time horizons $(\mathrm{TH})$ in the optimization problem and finds the optimum time-basis $\mathrm{TH}$.

In addition to the unacceptably long optimization time of previously developed MPC controllers, a few MPC models have focused on the prominent role of HVAC system operating time step (TS) in optimization functions. To fill this technical gap, this chapter describes the development of an advanced controller to control the performance of different HVAC system components using 
different operating time steps. Taking advantage of an exhaustive search method, this novel advanced controller can find the optimum time-varied HVAC system operating TS. This advanced controller also allows the MPC controller to employ different energy conservation strategy planning models (SPMs) on the HVAC system.

\subsection{Model-based Predictive Controller Development}

This section discusses the development of Demand MPC controller in detail. This section consists of System Description (Section 5.2.1), Modeling of Energy Technologies (Section 5.2.2), and Model Predictive Controller Construction/Formulation (Section 5.2.3) sub-sections. In Section 5.2.1, the house thermal model as well as HVAC system model functions are described in detail while Section 5.2.2 describes the development of photovoltaic array, wind turbine, and battery system simplified model functions. Section 5.2.3 describes the construction and formulation of different executive functions of the Demand MPC controller.

\subsubsection{System Description}

\subsubsection{House Thermal Model Function}

House model (zone) function consists of different physics-based equations and takes forecast weather dataset and HVAC supplied thermal energy $\left(\mathrm{Q}_{\mathrm{su}}(\mathrm{t})\right)$ as feed-in/input data in order to calculate the entire house's thermal demand and subsequently zone temperature. In this chapter, a simplified lumped thermal-network model has been developed and used for modeling the zone. Different heat conduction/transfer equations including walls and windows heat conductions $\left(\mathrm{Q}_{\mathrm{wl}}\right.$ (t) and $\mathrm{Q}_{\mathrm{wd}}(\mathrm{t})$, respectively), solar heat gain $\left(\mathrm{Q}_{\mathrm{so}}(\mathrm{t})\right)$, external surfaces sol-air temperatures, appliance/lighting heat gains $\left(\mathrm{Q}_{\mathrm{al}}(\mathrm{t})\right)$, and infiltration heat loss $\left(\mathrm{Q}_{\inf }(\mathrm{t})\right)$ are considered in the house model (zone) function for calculating the house thermal demand $\left(\mathrm{Q}_{z}(\mathrm{t})\right)$. The developed house thermal model considers the effects of solar radiation, outdoor temperature, wind speed, house location and orientation as the most important outdoor disturbances in the house thermal demand calculation; however, it is still simple enough to be implemented in MPC models. The main principles and physics-based equations used for developing the model are described here. 


\section{Walls Heat Conduction Equations:}

Equation (5-1) is used to calculate walls heat conduction [5.30].

$\mathrm{Q}_{\mathrm{wl}}(\mathrm{t})=\sum_{i=1}^{N_{1}} U_{w l_{i}} A_{w l_{i}}\left(T_{w l_{i}}-T_{z}\right)$

In this equation, $T_{w l_{i}}$ and $T_{z}$ indicate the outside wall surface and zone temperatures $\left({ }^{\circ} \mathrm{C}\right)$, respectively, and $N_{1}$ and $A_{w l}$ denote the number and area $\left(\mathrm{m}^{2}\right)$ of walls. The overall heat conduction coefficient of each wall is indicated by $\mathrm{U}\left(\frac{\mathrm{W}}{\mathrm{m}^{2} \mathrm{C}}\right)$. This equation is also used for calculating the heat conduction of roof and house floor.

The surface temperature of each wall exposed to solar radiation and the resulting heat flow into the house depend on the combined effects of several factors including the striking/incident radiation, surface absorptivity, outdoor/ambient air temperature, and wind speed next to the surface in question. This combined effect is expressed quantitatively by the sol-air temperature. For the surface of given envelope element, sol-air temperature is a theoretical external air temperature, which produces the same thermal effects on the element as the existing combination of the incident radiation and ambient air conditions. Therefore, it would produce the same external surface temperature, heat flow into and across the element, and internal temperatures.

The general equation of the sol-air temperature is described in Equation (5-2) [5.31]:

$T_{s a_{i}}=T_{o}+\alpha_{i} * \frac{I}{h_{o_{i}}}-L W R_{i}$

where $T_{s a}$ and $T_{o}$ indicate sol-air and outdoor temperatures $\left({ }^{\circ} \mathrm{C}\right)$, respectively, and $\alpha$ denotes the absorptivity of the external surface which depends on its color. For example, $\alpha$ can be considered 0.8 for a very dark color and 0.25 for a very light color. Suffix i in Equation (5-2) shows the number assigned to each wall. In this equation, $\mathrm{I}, h_{o}$, and $L W R$ indicate intensity of incident solar radiation on the surface $\left(\frac{W}{m^{2}}\right)$, overall external surface coefficient $\left(\frac{W}{m^{2}{ }^{\circ} \mathrm{C}}\right)$, and temperature drop due to longwave radiation, respectively. $h_{o}$ can be considered $20 \frac{\mathrm{W}}{\mathrm{m}^{2}{ }^{\circ} \mathrm{C}}$ for a typical exterior wall surface when the wind speed next to the wall is $3.5 \frac{\mathrm{m}}{\mathrm{s}}$. However, $h_{o}$ changes to $13 \frac{\mathrm{W}}{\mathrm{m}^{2}{ }^{\circ} \mathrm{C}}$ when the surface is exposed to a wind speed of $1.8 \frac{\mathrm{m}}{\mathrm{s}}$. To this end, $h_{o}$ can be calculated using the following equation [5.31]: 
$h_{o}=4.1 * W_{s}+5.5$

where $W_{s}$ indicates the wind speed $\left(\frac{m}{s}\right) . L W R$ changes between $4^{\circ} \mathrm{C}$ and $0^{\circ} \mathrm{C}$, respectively, in arid climate with a clear sky and cloudy conditions.

It should be noted that the intensity of incident solar radiation on the wall surface (I) is severely affected by the surface tilt angle and azimuth angle (orientation). In developing house thermal model, vertical walls (tilt angle $=90^{\circ}$ ) have been considered for the house and wall orientations were determined using Table 5-1. Incident solar radiation for horizontal surface was also envisaged for the roof.

Table 5-1: Azimuth angles for different walls of the house

\begin{tabular}{|c|c|}
\hline North Azimuth & $0^{\circ}$ \\
\hline West Azimuth & $-90^{\circ}$ \\
\hline South Azimuth & $180^{\circ}$ \\
\hline East Azimuth & $90^{\circ}$ \\
\hline
\end{tabular}

In the model development, CanMETEO software [5.32] has been utilized to calculate the specific intensity of incident solar radiation on each wall surface considering its tilt and azimuth angles. CanMETEO provides High Resolution Deterministic Prediction (HRDPS) forecasts developed by NRCan. These forecast information are calculated based on the house location including the house latitude, longitude, and altitude. This software considers the effects of cloud cover on the calculated incident solar radiation.

After calculating $T_{s a_{i}}, T_{w l_{i}}$ is calculated by Equation (5-4).

$T_{w l_{i}}=T_{s a_{i}}$

Windows Heat Conduction Equations:

Equation (5-5) is used to calculate windows heat transfer [5.30].

$\mathrm{Q}_{\mathrm{wd}}(\mathrm{t})=\sum_{i=1}^{N_{2}} U_{w d_{i}} A_{w d_{i}}\left(T_{w d_{i}}-T_{z}\right)$ 
In this equation, $T_{w d_{i}}$ indicates the windows exterior surface temperatures while $N_{2}$ denotes the number of windows in the zone/house. The same methodology used for calculating $T_{w l_{i}}$ is used to calculate $T_{w d_{i}}$. However, all the coefficents of Equations (5-2), (5-3), and (5-5) have been determined based on the windows manufacturer datasheets.

\section{Solar Heat Gain Equation:}

Equation (5-6) is used to calculate the solar heat gain [5.33].

$\mathrm{Q}_{\text {so }}(\mathrm{t})=A \times S H G F_{\text {max }} \times S H G C \times 1.15 \times C L F$

In this equation, $A$ indicates the un-shaded area of windows' glass; $S H G F, S H G C$, and CLF indicate the solar heat gain factor, solar heat gain coefficient, and cooling load factor, respectively. SHGF is the total solar heat transmission, which includes both directly transmitted energy and indirectly transferred heat, and depends on orientation of the windows. SHGF $\max$ is the maximum solar heat gain factor for windows. The solar heat gain factor is calculated using CanMETEO software. SHGC is the measure of the fraction of incident solar radiation that actually enters the entire windows. This coefficient is determined based on the energy absorbed by the glazing and is given by the windows' manufacturer. CLF indicates the time lag created based on the building thermal mass in absorbing the heat generated by external sources. This parameter can be found in ASHRAE Database [5.34].

\section{Appliance/lighting Heat Gain}

In this project, the temporal internal heat gain of appliances/lighting system $\left(\mathrm{Q}_{\mathrm{al}}(\mathrm{t})\right)$ is determined based on a schedule prepared using a typical standard load profile of residential houses [5.35].

\section{Infiltration Heat Transfer}

There are five different types of heat losses in a house: transmission (conduction), infiltration (convection), ventilation (convection), radiation, and moisture migration. The transmission heat losses take place in walls, roof, floor, and windows that are described in Equations (5-1) and (55). The uncontrolled introduction of outside air into a building results in natural infiltration heat loss. There are three methods including crack method, air change method, and average method 
which are used for calculating the natural infiltration heat loss. The air change method is described in Equation (5-7) [5.36]:

$\operatorname{Qinf}(\mathrm{t})=1.21 \times A C H_{\text {avg }} \times V \times\left(T_{z}-T_{o}\right)$

In this equation, $1.21\left(\mathrm{kj}^{3} \mathrm{~m}^{3}{ }^{\circ} \mathrm{K}\right)$ is the heat capacity of air at sea level while $A C H_{\text {avg }}$ and $\mathrm{V}$ denote annual average air exchange rate and house volume, respectively. $A C H_{\text {avg }}$ rate is calculated using Equation (5-8) [5.37]:

$A C H_{\text {avg }}=\frac{A C H_{50}}{N_{0}+H_{1}+S_{2}+L_{3}}$

where $\mathrm{ACH}_{50}$ indicates the hourly air change rate at $50 \mathrm{~Pa}$ and $\mathrm{N}_{0}$ represents the leakage-infiltration ratio as a site climate indicator. This site climate indicator accounts for the environmental and physical properties of a single-family house and is selected as $N_{0}=19$ for Toronto [5.37]. In order to correct $N_{0}$ indicator for a particular house, different correction factors including building height $\left(\mathrm{H}_{1}\right)$, site shielding $\left(\mathrm{S}_{2}\right)$, and leak type $\left(\mathrm{L}_{3}\right)$ can be used. These correction factors change based on the properties of the house. Table 5-2 has been prepared and developed in Sherman [5.38] and shows the rates of these correlation factors.

Table 5-2: Rates of correlation factors based on house properties/characteristics (Sherman [5.38])

\begin{tabular}{|lc|lr|lc|l|l|}
\hline \multicolumn{2}{|l|}{ Airtightness at 50 Pa [5.39] } & \multicolumn{2}{|l|}{ Height correction factor } & \multicolumn{2}{l|}{ Shielding correction factor } & \multicolumn{2}{l|}{ Leakiness correction factor } \\
\hline House Type & ACH50 & Number of stories & $\mathbf{H}_{\mathbf{1}}$ & Shielding Condition & $\mathbf{S}_{\mathbf{2}}$ & Leakiness Condition & $\mathbf{L}_{\mathbf{3}}$ \\
Loose & 10.35 & 1 & 1 & Well shielded & 1.2 & Small cracks & 1.4 \\
Average & 4.55 & 1.5 & 0.9 & Normal & 1 & Normal & 1 \\
Present & 3.57 & 2 & 0.8 & Exposed & 0.9 & Large holes & 0.7 \\
Energy-Efficient & 1.5 & 3 & 0.7 & & & & \\
\hline
\end{tabular}

After calculating all heat losses and gains, house thermal demand is calculated by the following equation:

$Q_{z}(t)=Q_{s u}(t)+Q_{w l}(t)+Q_{w d}(t)+Q_{s o}(t)+Q_{a l}(t)+Q_{i n f}(t)$ 
After calculating the house thermal demand, the next step of modeling the house thermal energy is to calculate the zone temperature deviation. Zone temperature deviation is significantly affected by the house thermal mass. Equation (5-10) shows the basic relationship between the thermal mass and the zone temperature deviation:

$\Delta Z T(t)=\frac{Q_{Z}(t)}{T M}$

where TM indicates the house total thermal mass material and is calculated using the following equation:

$T M=S H \times$ density $\times V_{Z}$

where $S H$, density, and $V_{Z}$ represent the specific heat, density of the thermal mass material and volume of the house, respectively. If only air is considered for the house thermal mass material, the zone temperature deviation is calculated by Equation (5-12):

$\Delta Z T(t)=\frac{\mathrm{Q}_{z}(\mathrm{t})}{c_{\text {air }} \times \rho_{\text {air }} \times V z}$

In Equation (5-12), $\rho_{\text {air }}$ indicates the density of air $\left(\mathrm{kg} / \mathrm{m}^{3}\right)$ and $c_{\text {air }}$ is the specific heat of air $=$ 1.006 (kJ/kg.K). The density of air varies between 1.534 to $1.12 \mathrm{~kg} / \mathrm{m}^{3}$ when air temperature changes between $-50^{\circ} \mathrm{C}$ to $40^{\circ} \mathrm{C}$. Equation (5-13) is used for calculating the density of air $\left(\rho_{\text {air }}\right)$ [5.40]:

$\rho_{\text {air }}=7^{*} 10^{-8} A T^{3}+8^{*} 10^{-6} A T^{2}-4.6^{*} 10^{-3} A T+1.293$

In Equation (5-13), AT indicates the dry-bulb temperature of the air. Since during the winter season the zone temperature should be kept within ASHRAE Comfort Standard (between $20^{\circ} \mathrm{C}$ and $24^{\circ} \mathrm{C}$ ), in this chapter, $\rho_{\text {air }}$ is considered $1.20 \mathrm{~kg} / \mathrm{m}^{3}$ as a constant value. Having $\rho_{\text {air }}$, zone temperature is calculated by using Equation (5-14) in the next time interval:

$Z T(t+1)=Z T(t)+\Delta Z T(t)$

The calculated zone temperature is fed to the HVAC model function for further calculation as well as making a decision about turning the HVAC system on/off in the next time step. House A is the 
case study house where the developed Demand MPC controller is applied. This Archetype House has been constructed by the Toronto and Region Conservation Authority (TRCA) [5.41, 5.42]. This house demonstrates sustainable housing technologies through experimentation and research and was one of the first Canadian projects achieved a Leadership in Energy and Environmental Design (LEED) for Homes Platinum Certification [5.41]. All the parameters/coefficients of the developed house thermal model are adjusted based on House A building characteristics as described in Table 5-3:

Table 5-3: House thermal model parameters/coefficients description

\begin{tabular}{|c|c|c|c|c|c|c|}
\cline { 2 - 7 } \multicolumn{1}{c|}{} & \multicolumn{2}{c|}{ Wall } & \multicolumn{2}{c|}{ Windows } & \multicolumn{2}{c|}{ Infiltration Coefficient } \\
\hline Orientation & Area $\left(\mathrm{m}^{2}\right)$ & $\mathrm{U}\left(\frac{\mathrm{W}}{\mathrm{m}^{2}{ }^{\circ} \mathrm{C}}\right)$ & $\mathrm{Area}\left(\mathrm{m}^{2}\right)$ & $\mathrm{U}\left(\frac{\mathrm{W}}{\mathrm{m}^{2}{ }^{\circ} \mathrm{C}}\right)$ & Factor & Value \\
\hline South & 39.73 & 0.177 & 11.04 & 1.69 & $\mathrm{ACH}_{50}$ & 1.5 \\
\hline North & 39.73 & 0.177 & 9.59 & 1.69 & $\mathrm{H}_{1}$ & 0.8 \\
\hline East & 65.32 & 0.177 & - & - & $\mathrm{S}_{2}$ & 1 \\
\hline West & 65.32 & 0.177 & 10.33 & 1.69 & $\mathrm{~L}_{3}$ & 1.4 \\
\hline Floor(slab) & 80.17 & 0.565 & - & - & volume $\left(\mathrm{m}^{3}\right)$ & 932.5 \\
\hline Roof & 46.44 & 0.142 & - & - & & \\
\hline
\end{tabular}


Figure 5-1 illustrates solar radiation (SR) on different orientations of House A during winter (heating) season while Figure 5-2 shows the outdoor air temperature and wind speed during this period. The information in Figures 5-1 and 5-2 have been obtained from metropolitan Toronto weather available at the TRNSYS library.
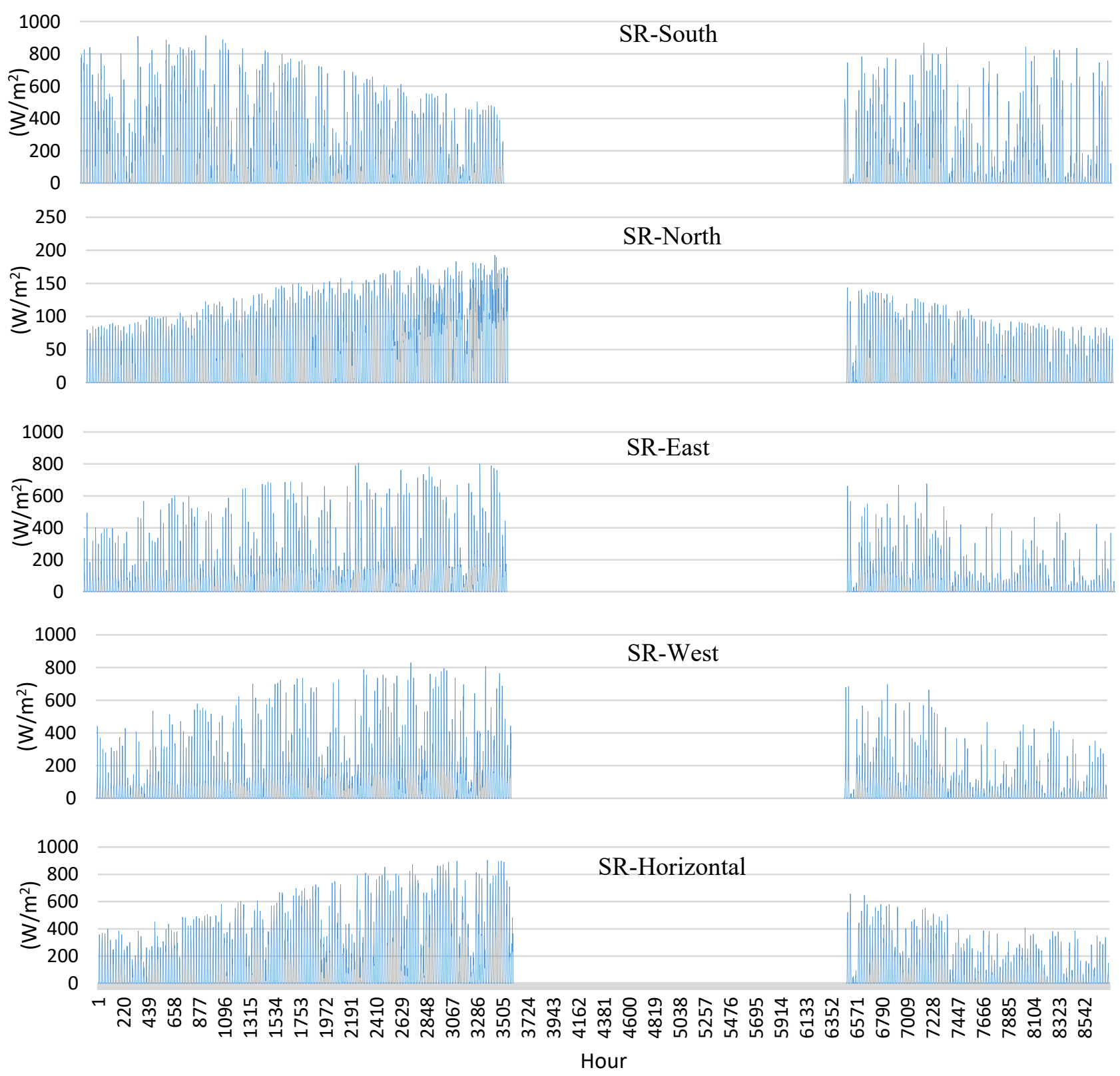

Figure 5-1: Solar radiation (SR) on different orientations of House A during heating season 


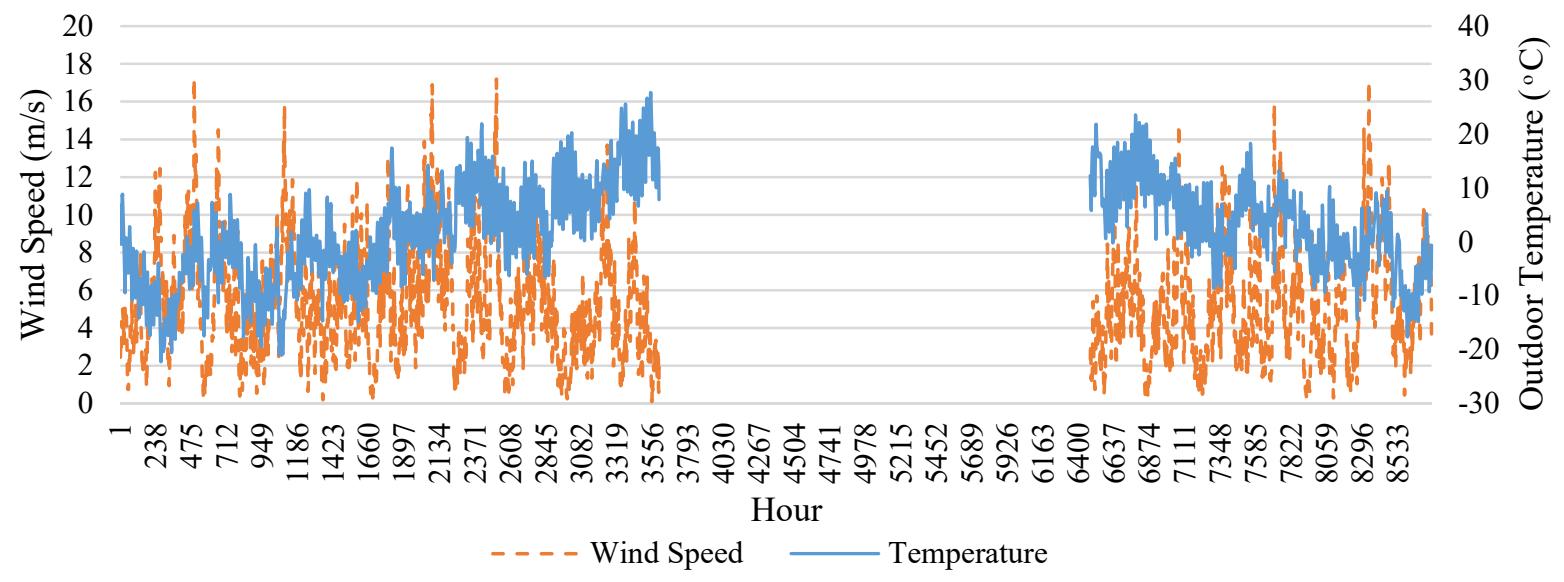

Figure 5-2: Outdoor air temperature and wind speed during the heating season

The simplified house model has been run based on metropolitan Toronto historical weather data (obtained from the TRNSYS library) to calculate the hourly house thermal demand $\left(Q_{z}\right)$ during the heating season. The calculated hourly house thermal demand is compared with the result of House A TRNSYS calibrated model [5.43], using Equation (5-15), to examine the accuracy of the simplified house model.

Correlation Coefficient $=\frac{\operatorname{Cov}\left(S_{1}, S_{2}\right)}{\sigma_{S_{1}} * \sigma_{S_{2}}}$

Correlation coefficient (CC) computes the dependent relationship between two series of data [5.44]. In Equation (5-15), $S_{1}$ and $S_{2}$ represent first and second series of data while Cov and $\sigma$ indicate covariance function and standard deviation of each series of data $\left(\sigma_{S_{1}}=1.86, \sigma_{S_{1}}=1.81\right)$. It was found that the simplified house model and calibrated TRNSYS model results (illustrated in Figure 5-3) were highly correlated; the correlation coefficient was 0.97. 


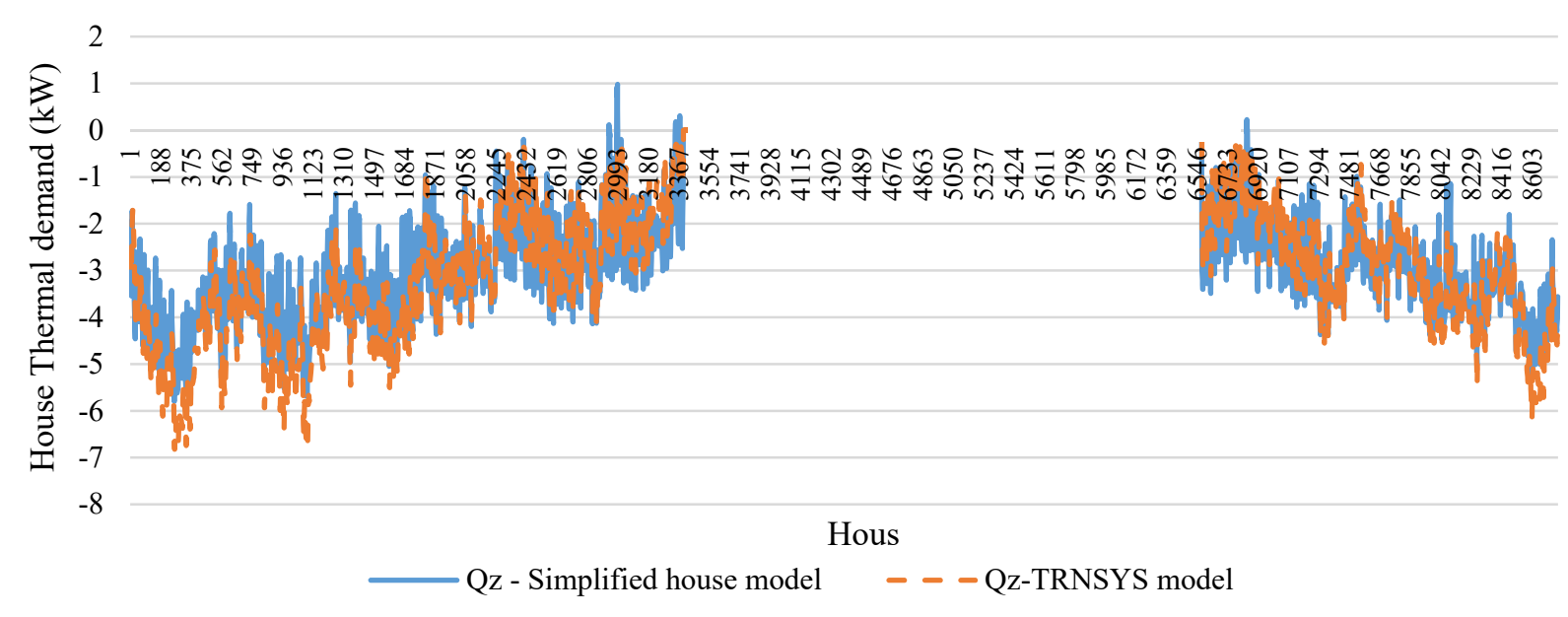

Figure 5-3: House thermal demand (Qz) during the heating season

\subsubsection{HVAC Model Function}

House A HVAC system consists of four major components including heat recovery ventilator (HRV), air handling unit (AHU), air source heat pump (ASHP), and wall-mounted natural gas fired mini boiler (MB) along with a simple thermostat which controls HVAC system by sending on/off commands. A local/internal controller has been designed for the AHU by the manufacturer. This internal controller is set up to run ASHP as the primary hot air supplier and MB as the backup system.

Different approaches are used for modeling the components of HVAC system. These approaches can be classified as white-box, black-box, and gray-box approaches. The white-box (physicsbased) approaches, also known as "analytical first principal models," are the first approach used for constructing a process model. These models are established according to the detailed process knowledge and governing laws of underlying physical phenomena/platforms and principles. In the black-box (data driven) model, the performance data of the system collected by implementing normal or specific tests, is used to find the relationship between input and output variables of the system taking advantage of different mathematical methods/techniques. Since the model is estimated based on the real performance data, this modeling approach offers the best accuracy in comparison to other techniques. In the third approach, the gray-box method, the basic structure of the model is founded using the physics-based methods. The parameters of the model are determined by using different estimation algorithms on the measured/collected data of the system. 
Physics-based equations have been used for calculating the supplied thermal energy of different components of HVAC system. However, since in House A the operational data of the HVAC system are consistently recorded in the data acquisition (DAQ) system, black-box (regression model) approach has been used for predicting the behavior of some HVAC system variables. A sub-function is designed for modeling each component containing black-box and physics-based equations.

\section{HRV Sub-function}

HRV exchanges heat between the outgoing exhaust air stream and incoming fresh air. HRV unit comprises of one inlet air stream and one outlet air stream. In total, there are two input and output altogether, for which the temperature of input (fresh outdoor air temperature $-\mathrm{AT}_{\mathrm{fo}}$ ) and output (preheated fresh air temperature - $\mathrm{AT}_{\mathrm{pf}}$ ) are measured.

Figure 5-4 shows HRV component/sub-function comprising its inputs and outputs. HRV subfunction is constructed using the following equations [5.37, 5.45]:

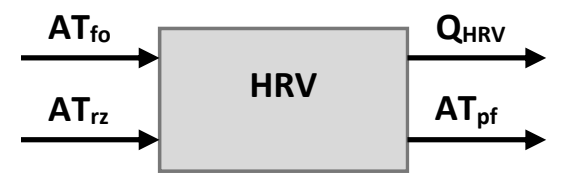

Figure 5-4: HRV component/subfunction

$$
\begin{aligned}
& \eta_{s}=\frac{A T_{p f}-A T_{f o}}{A T_{r z}-A T_{f o}} \\
& Q_{\max }(k W)=\text { Maximum available heat for recovery }
\end{aligned}
$$$$
Q_{H R V}(k W)=Q_{\max }(k W) \times\left(1-\eta_{s}\right)
$$

where $\eta_{S}$ and $Q_{H R V}$ indicate the sensible thermal efficiency and HRV recovered thermal power $(\mathrm{kW})$, respectively, and $\mathrm{AT}_{\mathrm{rz}}$ represents the returned air temperature coming from the zone. The HRV data during the first five days of February 2015 was selected for modeling the HRV. February is a cold month in Ontario, therefore, outdoor air temperature $\left(\mathrm{AT}_{\mathrm{fo}}\right)$ varied between $20^{\circ} \mathrm{C}$ and $0^{\circ} \mathrm{C}$. The returned air temperature coming from the zone $\left(\mathrm{AT}_{\mathrm{rz}}\right)$ was around $22^{\circ} \mathrm{C}$ and was regulated by the AHU. The preheated fresh temperature $\left(\mathrm{AT}_{\mathrm{pf}}\right)$ varied between $16^{\circ} \mathrm{C}$ and $22^{\circ} \mathrm{C}$, and was transmitted directly to the AHU. The flow rate of supply and return air $\left(A F_{H R V}\right)$ was almost constant around $0.058 \mathrm{~kg} / \mathrm{s}$.

After selecting the samples, the trendline between input and output data of HRV is modelled using a simple linear regression method to make a consistency in MPC model and to increase the linear optimization speed. Figure 5-5 shows the relationship between cold outdoor air temperatures and 
pre-heated fresh air temperature. Based on the trendline in Figure 5-5, $\mathrm{AT}$ pf is calculated based on $\mathrm{AT}_{\text {fo }}$ using the following linear equation:

$A T_{p f}=0.203 A T_{f o}+20.65$

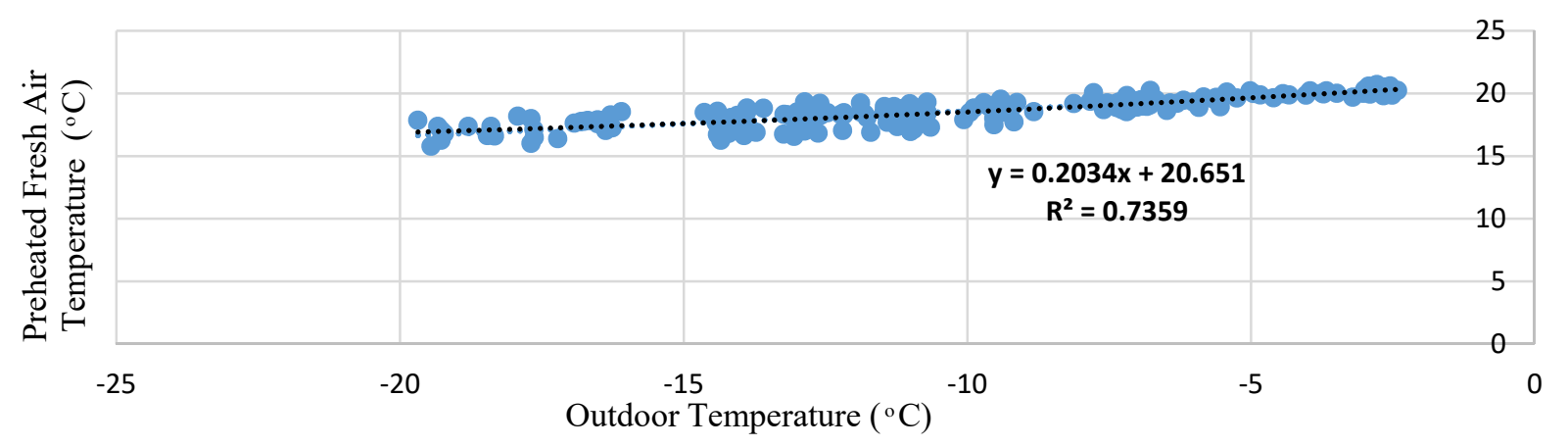

Figure 5-5: Trendline between cold outdoor air temperatures and preheated fresh air temperature - HRV

\section{Validation of Simulated Data}

In Figure 5-6, simulated data generated using Equation (5-18) is plotted versus measured data. To this end, 0.85 is calculated as the correlation coefficient that shows the accuracy of the HRV subfunction.

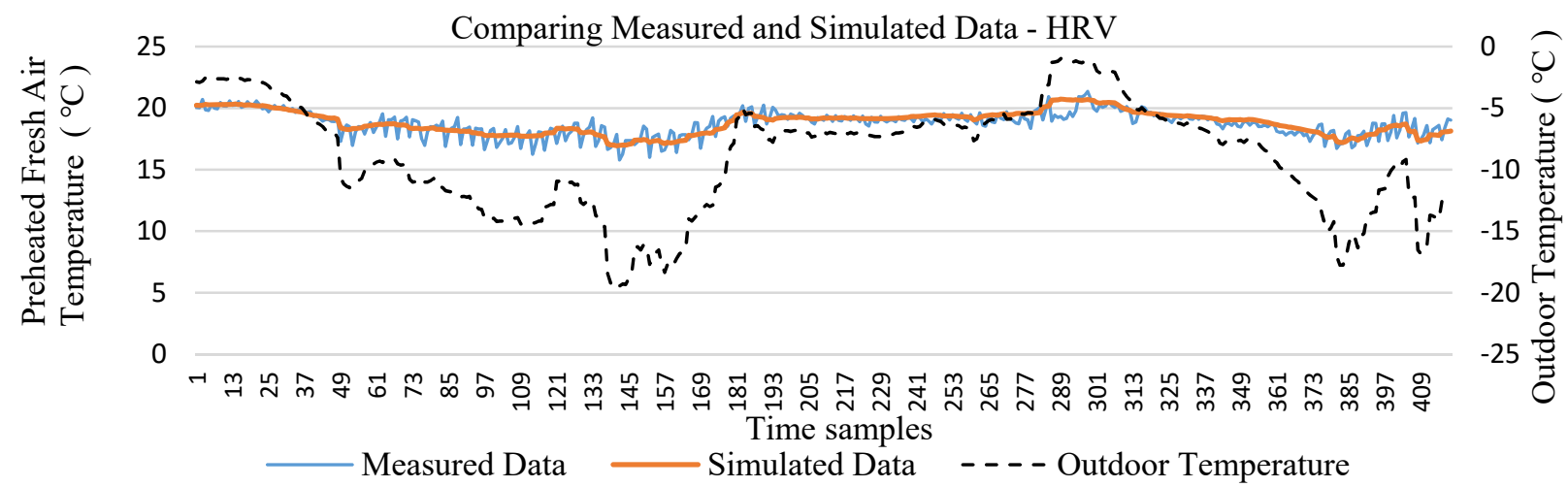

Figure 5-6: Validating HRV simulated data 


\section{AHU Sub-function}

AHU of TRCA Archetype House A serves the whole house as one zone. The AHU works during the summer and winter seasons to supply cold and warm air to the zone. Fresh outdoor air, which is pre-cooled or pre-heated through HRV, enters the inlet of the AHU along with the return air stream from the zone. The mixed air, consisting of return and pre-heated fresh outdoor air streams, passes through the ASHP evaporator/condenser inside the AHU where the mixed air transfers heat to or absorbs heat from. The AHU has two inlet air streams comprising of one pre-heated/cooled fresh air stream from the HRV and the return air stream from the zone.

Figure 5-7 shows AHU component/sub-function containing its inputs and output. This subfunction is constructed using the following equations [5.46]:

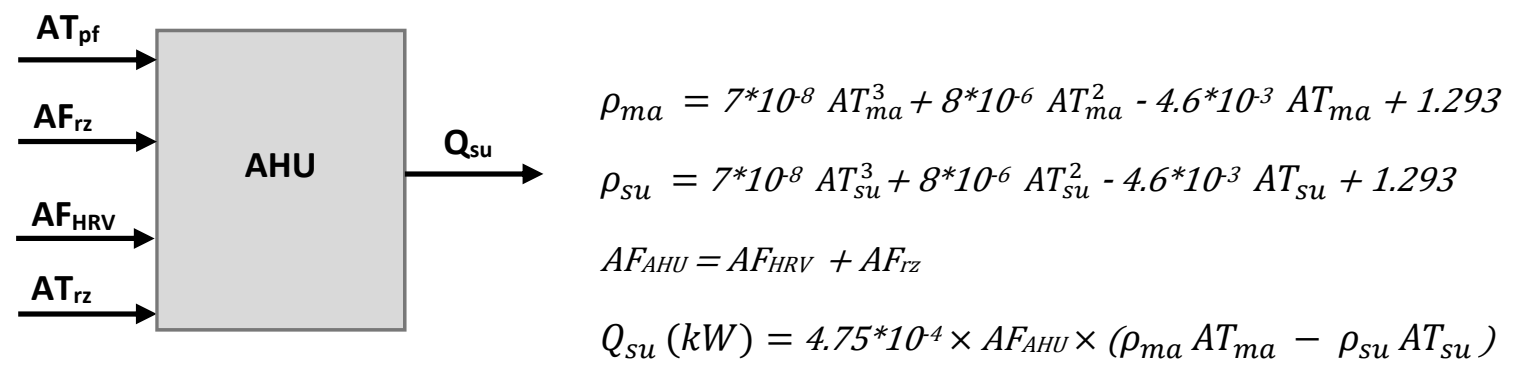

Figure 5-7: AHU component/subfunction

where $Q_{s u}$ indicates the AHU supplied thermal power $(\mathrm{kW})$ and $\mathrm{AT}_{\text {ma }}$ and $\mathrm{AT}_{\text {su }}$ represent the temperature of mixed air and supplied air temperature of AHU, respectively. $\rho_{m a}$ and $\rho_{s u}$ show the air density in the mixing box (mixed air) and in the outlet of AHU, respectively. $A F_{r Z}$ and $A F_{H R V}$ indicate the returned zone and HRV air flows, while $A F_{A H U}$ presents the supplied air flow at the outlet of AHU.

$4.75^{*} 10^{-4}$ coefficient in Equation (5-22) is calculated using the following equation:

$4.75^{*} 10^{-4}=$ air specific heat $(1.006 \mathrm{~kJ} / \mathrm{kg} . \mathrm{K}) \times$ air flow rate $\left(C F M=1 / 2119 \mathrm{~m}^{3} / \mathrm{sec}\right)$

The inlet air streams are assumed to be well mixed inside the AHU, and the temperature of mixed air (ATma $)$ in the mixing box is calculated based on the mixing box model as follows: 
$A T_{m a}=\frac{m_{f a} T_{f a}+m_{r a} T_{r a}}{m_{f a}+m_{r a}}$

where $\mathrm{T}$ is the temperature and $\mathrm{m}$ is the flow rate and the subscripts 'ma', 'fa', and 'ra' represent mixed air, fresh air, and return air, respectively. As discussed before, the air flow rate of HRV as the inlet of AHU is almost constant around $0.058 \mathrm{~kg} / \mathrm{s}$. Based on AHU outlet air flow (AFAHU) sensor information, the flow rate of supplied AHU air during HVAC operation is almost constant around $0.2757 \mathrm{~kg} / \mathrm{s}$. In the Demand MPC controller, AHU component comprises one inlet air stream, which is the output of mixing box, and one outlet air stream. $T_{m a}$ is calculated based on the measured temperature of $\mathrm{AT}_{\mathrm{pf}}$ and $\mathrm{AT}_{\mathrm{rz}}$. Outlet air stream temperature is determined by measuring $\mathrm{AT}_{\text {su }}$ sensor information.

The same methodology used for modeling the HRV has been used for modeling AHU. As a result, AHU sub-function has been constructed using the following linear equation:

$A T_{s u}=2.86 T_{m a}-30.20$

\section{ASHP Sub-function}

House A has an electric two-stage variable capacity ASHP and a natural gas mini boiler to generate warm air through the AHU to meet the space heating demand. ASHP maintains the zone temperature in both summer and winter seasons. In the summer season, it takes the warm air at its inlet and returns the cold air to the AHU to be transferred to the zone. In reverse order, in the winter season, it takes the cold air at its inlet and returns the warm air to the AHU. Figure 5-8 shows ASHP sub-function containing its inputs and output. ASHP sub-function is constructed using the following equation:

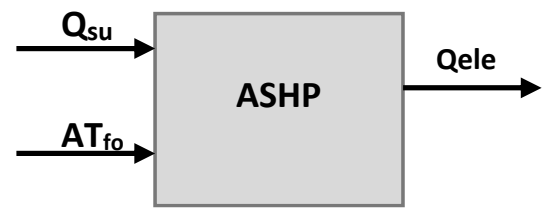

$$
Q_{\text {ele }}(k W)=\frac{Q_{s u}}{c o p}
$$

Figure 5-8 : ASHP component/subfunction

where cop indicares the ASHP coefficent of performance and $Q_{e l e}$ indicates the ASHP electricity demand $(\mathrm{kW})$ which would play a key role in the decision making/optimization process later. 
Outdoor temperature has a direct impact on ASHP coefficient of performance. The ASHP model, developed in [5.47], has been used in this project as ASHP sub-function. As a result, ASHP subfunction is presented by the following linear equation where $A T_{f o}$ indicates the outdoor air temperature:

$C O P=0.115 A T_{f o}+3.72$

\section{MB Sub-function}

The efficiency of the mini boiler is determined based on the flow rate of water (load percentage (LP)) circulating through the boiler. Figure 5-9 illustrates the mini boiler efficiency curve provided by the manufacturer.

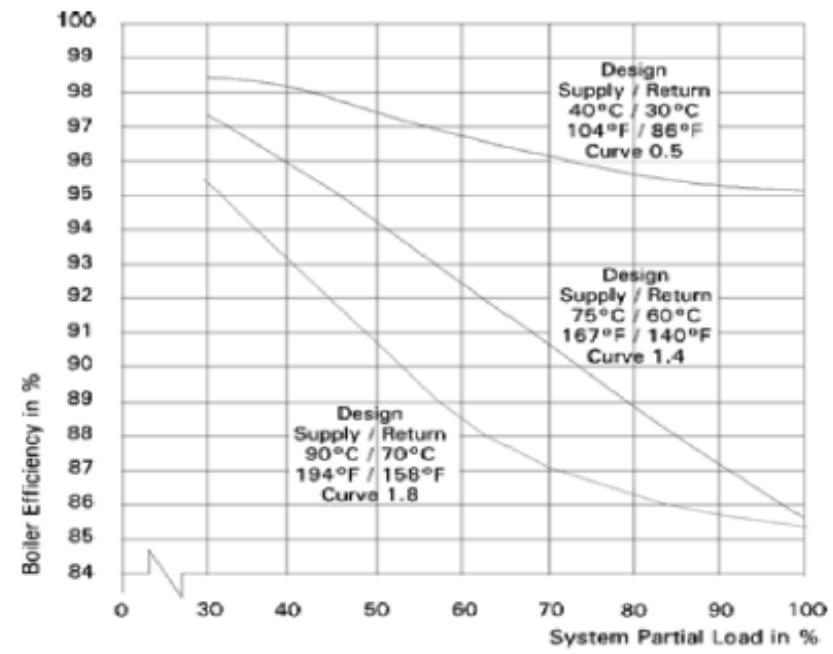

Figure 5-9: Mini boiler efficiency curves provided by the manufacturer [5.48]

Based on curve 1.8 in Figure 5-9 (which corresponds to the House A installed mini boiler), the following linear function is estimated for calculating the mini boiler efficiency:

$\eta_{m b}=-0.15 P L+98.66$

where PL indicates the partial load. Figure 5-10 shows MB component/sub-function containing its inputs and output. MB sub-function is constructed using the following equation: 


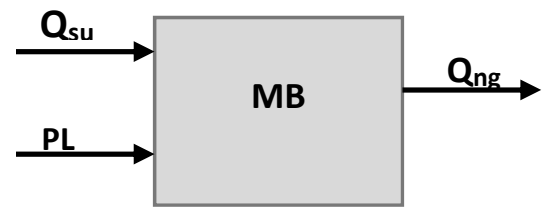

$$
Q_{n g}(k W)=\frac{Q_{s u}}{\eta_{m b} \times 10.3}
$$

Figure 5-10: MB component/sub-function

In Equation (5-29), 10.3 constant is used for converting $1 \mathrm{~m}^{3}$ of natural gas energy content into $\mathrm{kW}$. Figure 5-11 represents a schematic diagram of House-HVAC integrated model in which the connections among different components of HVAC system and the connections between the house thermal model and HVAC system components are shown.

House - HVAC Integrated Model Schematic Diagram

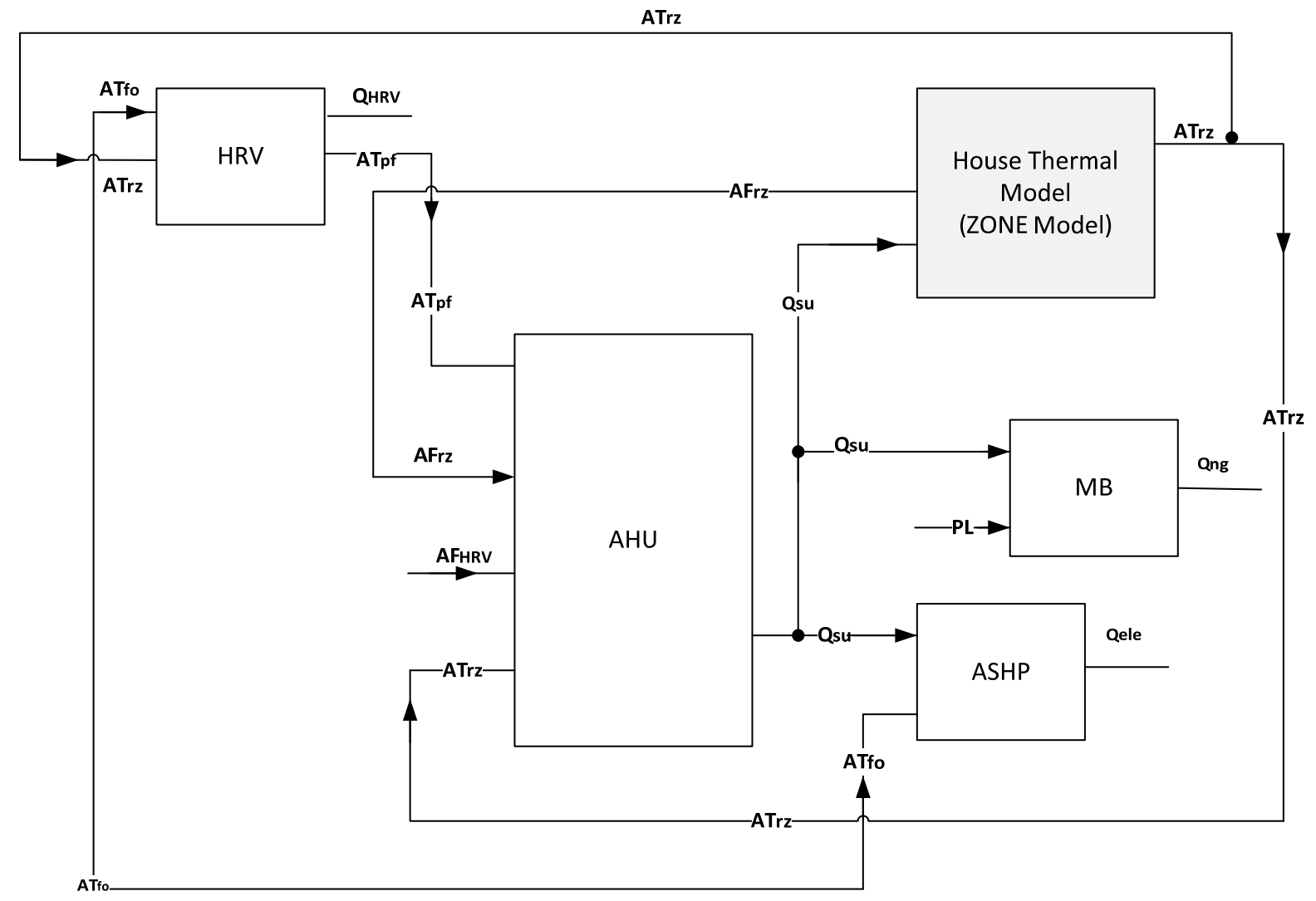

Figure 5-11: House-HVAC integrated model schematic diagram 


\subsubsection{Modeling of Energy Technologies}

\subsubsection{Photovoltaic Array Model}

The model developed in [5.49] has been adapted and implemented here to calculate the photovoltaic array output power. In this model, solar irradiance $\left(G_{P}\right)$ on tilted surface, when $30^{\circ}$ and $180^{\circ}$ are selected as tilt and azimuth angles, and wind speed $\left(W_{s}\right)$ are calculated using CanMETEO software.

$\Delta T C_{\text {wind }}=I * G_{P}+J * W_{s}$

$T C=O T+\frac{N C O T-20}{800} * G_{P}-\Delta T C_{\text {wind }}$

$I_{s c}=\left[I_{s c, s t c}+K_{I}\left(T C-25^{\circ}\right)\right] \frac{G_{P}}{1000}$

$V_{o s}=V_{o c, s t c}-K_{V} * T C$

$P_{P V}=N_{s} * N_{p} * I_{S C} * V_{o s} * F F$

where NCOT is the nominal cell operating temperature $\left({ }^{\circ} \mathrm{C}\right), O T$ is the outdoor temperature, $T C$ is the cell temperature, $I_{s c, s t c}$ and $V_{o c, s t c}$ are module short circuit current and open circuit voltage under STC condition, with $K_{V}$ and $K_{I}$ as their corresponding temperature coefficients; $I$ and $J$ as the coefficients determined with site location [5.50]. Based on Toronto latitude and longitude 0.005 and 0.1 are selected for $I$ and $J$ respectively. $P_{P V}$ indicates the power of the PV array with $N_{s}$ module in serial and $N_{p}$ module in parallel and FF is the fill factor of each module.

\subsubsection{Wind Turbine (WT) Model}

The wind kinetic energy is transformed into electric power by wind turbines. The WT output power can be mathematically calculated as follows [5.51]:

$P_{w}= \begin{cases}0, & v_{w}<V_{c i} \text { or } v_{w}>V_{c o} \\ P_{w, r} \frac{v_{w}-V_{c i}}{v_{r}-V_{c i}}, & V_{c i} \leq v_{w} \leq V_{r} \\ P_{w, r}, & V_{r} \leq v_{w} \leq V_{c o}\end{cases}$ 
where $v_{w}$ is the wind speed at the hub height, $V_{r}$ is the rated wind speed, $V_{c i}$ is the cut-in wind speed, $V_{c o}$ is the cut-out wind speed and $P_{w, r}$ is the rated WT output power. If the height of wind turbine hub is different, the power law engineering approximation is used for calculating the wind speed at that specific height [5.52]:

$$
v_{1}=v_{0}\left(\frac{H_{1}}{H_{o}}\right)^{\alpha}, \alpha=0.35
$$

where $v_{1}$ is the wind speed at hub height $H_{1}, v_{o}$ is the recorded wind speed at height $H_{o}$ and $\alpha$ is the power law exponent that varies from 0.1 to 0.6 depending on the surface roughness and stability of the atmosphere. The WT output power (in Watts) is calculated using the following equations:

$$
P_{w t}(t)=P_{v w}(t)\left[\left(1-f_{\text {turbulence }}\right)\left(1-\frac{\text { Altitude }}{152.4 \mathrm{~m}} f_{\text {airdensity }}\right], P_{v w}(t)=P_{w}, v_{w}=v_{1}\right.
$$

where $P_{v w}(t)$ is the power curve intercept at the corresponding wind speed, $f_{\text {turbulence }}$ is the turbulence factor (to account for air turbulence and site variability), Altitude is the elevation of the site and $f_{\text {airdensity }}$ is the air density factor (to account for changes from the sea-level performance).

The wind turbine available power used for supplying the load is calculated as:

$P_{W T}(t)=W_{n} P_{w t}(t) \eta_{i n v}$

where $W_{n}$ is the number of wind turbines installed, $P_{w t}$ is the output power of the wind turbine using its power curve and $\eta_{i n v}$ is the efficiency of the inverter.

\subsubsection{Battery Bank Model}

In each simulation time step the state of charge (SOC) of the battery bank is calculated using the following equation [5.49]:

$S O C_{t+1}=S O C_{t}+\mu_{B} \frac{P_{B}(t+1)}{C_{n}} * 100$

Where $C_{n}$ is the total nominal capacity of battery bank (Ah), $\mu_{B}$ is the battery round-trip efficiency and $P_{B}(t+1)$ is the power either charged in or discharged from the battery bank at time step $t+1$. 
Based on the result of House A TRNSYS calibrated model [5.43], $1.82 \mathrm{~kW}$ is estimated as the average of HVAC system energy demand during the heating season. Therefore, in the MPC model development, $2 \mathrm{~kW}$ is considered as the total capacity of renewable sources for covering the HVAC load. Table 5-4 shows the maximum capacity of renewable sources and total capacity of battery bank system.

Table 5-4: Maximum capacity of renewable sources and total capacity of battery bank system

\begin{tabular}{|l|c|c|c|}
\hline Energy Source & PV $(\mathrm{kW})$ & WT $(\mathrm{kW})$ & Battery Bank (kWh) \\
\hline Capacity & 1 & 1 & 10 \\
\hline
\end{tabular}

\subsubsection{Model-based Predictive Controller Construction/Formulation}

\subsubsection{Overview on the Methodology of MPC Controller}

A basic/preliminary overview on the methodology (configuration) of Demand MPC controller, including its intelligent algorithm that itself consists of two smart decision support systems, exhaustive/pure optimization schemes, and energy conservation strategy planning models (ECSPMs), is described in this section. The developed Demand MPC controller assists in estimating the optimum: time-varied control time horizon, HVAC system operating time step, and indoor set point profile. Having these optimum values/profiles minimized the HVAC system energy demand and/or maximized saving on HVAC system energy cost, without compromising thermal comfort.

Figure 5-12 describes the framework of Demand MPC controller. This figure depicts HouseHVAC integrated model (that includes Exhaustive Search optimization method), Arranged Indoor Set Point Generator (AISPG) and Comprehensive Set Point Matrix Generator (CSPMG) as two smart decision support systems that making the intelligent algorithm and Linear Optimization functions as the executive functions of Demand MPC controller. The descriptions of different decision support/optimization cores utilized in the Demand MPC controller (inside the corresponding executive functions), are given in "Decision Support/Optimization Cores" column of this figure. The concept, configuration, structure, and operational mechanism of each of the aforementioned executive functions are described in detail in Section 5.2.3.2. 
Based on Figure 5-12, downloading the forecast weather dataset (FWD) from the Meteorological Service of Canada (MSC) web portal (using CanMETEO software developed by Natural Resources Canada (NRCan)) is the first step of operational process. Having FWD ready, indoor set point temperature is set to $22^{\circ} \mathrm{C}$ for the whole day and House-HVAC integrated model (which is built up combining house thermal model and HVAC model functions) is run based on this initial set point and the downloaded weather dataset to estimate the HVAC system demand profile for the whole day. An exhaustive optimization core is designed inside the HVAC model function. This optimization core calculates the optimum HVAC operating time step in which the HVAC system demand is minimum considering the effects of the time-varied outdoor disturbances. The zone temperature is maintained within the ASHRAE Thermal Comfort Standard ranges during the optimization process. At the end of the optimization process, the HVAC model function feedsout/exports the optimal hourly HVAC system demand (kWh) as an output vector to be exploited later in the Arranged Indoor Set Point Generator (AISPG) function. AISPG function reveals a demand pattern from the feed-in vector in the first step and then utilizes a smart decision support system (Core 2) in the second step (taking advantage of normalization methods) to generate an hourly indoor set point pattern/profile for reducing the HVAC system energy cost by maximum exploitation of outdoor disturbances. To this end, the HVAC system is initiated to run at more favorable hours to store the thermal energy and subsequently take advantage of the stored thermal energy in non- or less-favorable hours (detail explanation presented in Section 5.2.3.2.3) in order to decrease the overall HVAC system demand and/or energy cost. The supplied arranged hourly indoor set points are fed-out/exported by AISPG function as an output vector to be utilized later in the Comprehensive Set Point Matrix Generator (CSPMG) function. CSPMG function is developed with the aim of incorporating more energy cost saving value into the arranged hourly indoor set points by utilizing pre-heating processes during off-peak hours. Since during the winter season there are three different switching points between TOU electricity prices with the potential of energy cost saving (switching from off-peak to peak hours at 7 am, switching from peak to midpeak hours at $11 \mathrm{am}$, and switching from mid-peak to peak hours at $5 \mathrm{pm}$ ), CSPMG function consists of three different sub-functions. Each sub-function is constructed to pre-heat the house during the hours in which the electricity price is less expensive by increasing the indoor set point during these hours. These sub-functions are also constructed to reduce the HVAC system demand during the hours in which the electricity price is more expensive by decreasing the set point 
temperature in these hours. Since a variety of set points can be selected in each particular hour for either pre-heating the house or reducing the HVAC system demand, a matrix containing all allowable hourly set point patterns (which confirm the ASHRAE Comfort Standard) is generated at the end of each sub-function. To generate this matrix, the second smart decision support system (Core 3) is developed to solve different probability problems. In the next step, a mechanism is developed in the CSPMG function to combine the three separate matrices together in order to build a comprehensive matrix which covers the whole control time horizon. The combination of AISPG and CSPMG functions builds an intelligent algorithm that significantly reduces the MPC controller optimization time by only extracting the system useful states through whose system states. The supplied comprehensive set point matrix is fed by CSPMG function to be utilized later in the House-HVAC integrated model.

In the next step, Demand MPC controller run House-HVAC integrated model function by importing supplied comprehensive set point matrix which contains a variety of hourly set point patterns. After running the House-HVAC integrated model function, a comprehensive HVAC demand matrix containing a variety of hourly HVAC demand patterns is generated. It should be noted that each hourly demand pattern in the comprehensive HVAC demand matrix is optimal in terms of HVAC system energy demand. This occurs because an optimum operating time step is selected in the HVAC function for each set point pattern by taking advantage of HVAC function built-in exhaustive optimization core.

The Demand MPC controller exports the developed comprehensive HVAC demand matrix into the Linear Optimization (LO) function to determine which optimum hourly demand pattern and corresponding hourly set point pattern generate the minimum energy cost considering time-varied outdoor disturbances in that specific control time horizon. Different criteria and features including TOU pricing scheme, supplied renewable energy (from photovoltaic panels and/or wind turbine, calculated based on FWD), the energy stored in battery bank, and solar community's surplus energy (along with their particular weighting factors) are considered in the linear optimization core. The entire aforementioned processes are re-proceed by Demand MPC controller for various control time horizons. All contents, dimensions and configurations of optimization/decision support cores are rearranged/reformatted for each control time horizon. With comparison the results of all control time horizons, it is determined which control time horizon offers the minimum 
HVAC energy system demand and cost based on the time-basis/temporal conditions such as outdoor disturbances and house HVAC component characteristics.

After finalizing the process of Demand MPC controller, the optimum control time horizons, their corresponding optimal HVAC system operating time steps, and indoor set point profiles, which lead to minimum HVAC system energy demand and cost, are presented in a control vector as the final output. 


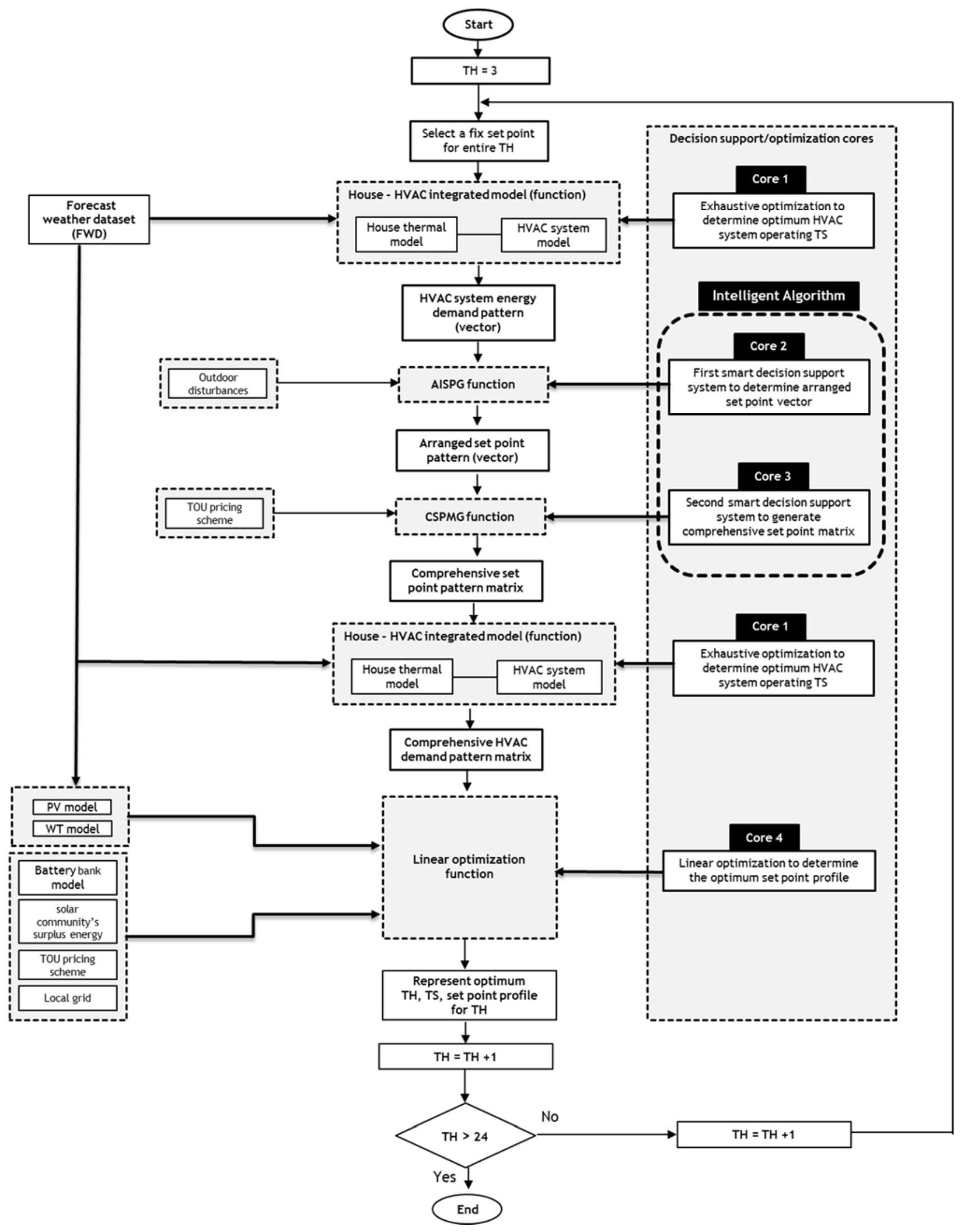

Figure 5-12: Framework of Demand MPC controller 


\subsubsection{Executive Functions}

\subsection{House-HVAC Integrated Model Function}

House-HVAC integrated model is the first executive function applied in the Demand MPC controller. This fundamental function is established by integrating the house thermal demand and HVAC system functions, which were described in Sections 5.2.1.1 and 5.2.1.2. In this executive function, zone temperature at the new time step, which was calculated based on the zone temperature deviation, is fed to the HVAC model function for further calculation as well as decision-making process. House-HVAC integrated model function consists of a fundamental subfunction called HVAC Smart Controller Function that plays a significant role in managing and optimizing HVAC system operation by detecting the optimum HVAC operation time step based on the exhaustive optimization function.

\subsection{HVAC Smart Controller Function}

The HVAC smart controller function plays fundamental roles in different areas including controlling the performance of HVAC system, performing exhaustive optimization, and implementing strategy planning models. This function returns optimum results such as minimum hourly ASHP/MB demand vectors, optimum HVAC system operating time step, maximum allowable time step, and zone temperature to the Demand MPC controller for further optimization and decision-making processes. The HVAC smart controller is designed based on the user-friendly concept. In other words, the HVAC smart controller's constraints can be set based on the user's preferences. To this end, minimum/maximum HVAC operating time steps as well as minimum/maximum zone temperatures are set in the controller based on the user's desire. The HVAC smart controller runs the House-HVAC integrated model on each simulation time step (minute). All simulation processes are adjusted with the control time horizon in the HVAC smart controller. The HVAC system operating/control time step changes during the minimum and maximum time steps that are defined by the user. However, due to the time-dependent conditions, increasing the time steps may lead to the zone temperature violation. In other words, by selecting larger time steps zone temperature may contravene the ASHARE Comfort Standard and/or the

minimum/maximum zone temperatures defined by the user. These time steps are rejected by the HVAC smart controller. The largest time step, which ensures zone temperature constants (which 
are set up based on the user's preferences), is selected and represented by the controller as the maximum allowable time step.

HVAC smart controller consists of different control loops. The first control loop is designed to check the zone temperature against its constraints. If the zone temperature meets the constraints, in the second control loop it will be compared with the given hour indoor set point (at each time step) to make a decision about turning the HVAC system on or off. Figure 5-13 depicts zone temperature oscillation by selecting 3-hour as the control time horizon, 18-minute as the HVAC operating time step and $22^{\circ} \mathrm{C}$ as a constant indoor set point. It should be noted that decision regarding turning the HVAC system on or off is only made in each control time step. Based on Figure 5-13, since zone temperature at 18-minute (the first decision making/checking point) is lower than the set point, the second control loop decided to turn on the HVAC system. As a result, the zone temperature rises up soon after turning the HVAC system on. However, since zone temperature is higher than the set point at 36-minute, 54-minute, and 72-minute (the second, third, and fourth control time steps, respectively), HVAC system is kept off during this period, by the second control loop, to decrease and maintain the zone temperature around the set point. HVAC system is turned on at 90 -minute as the fifth set point since the zone temperature fell below the indoor set point.

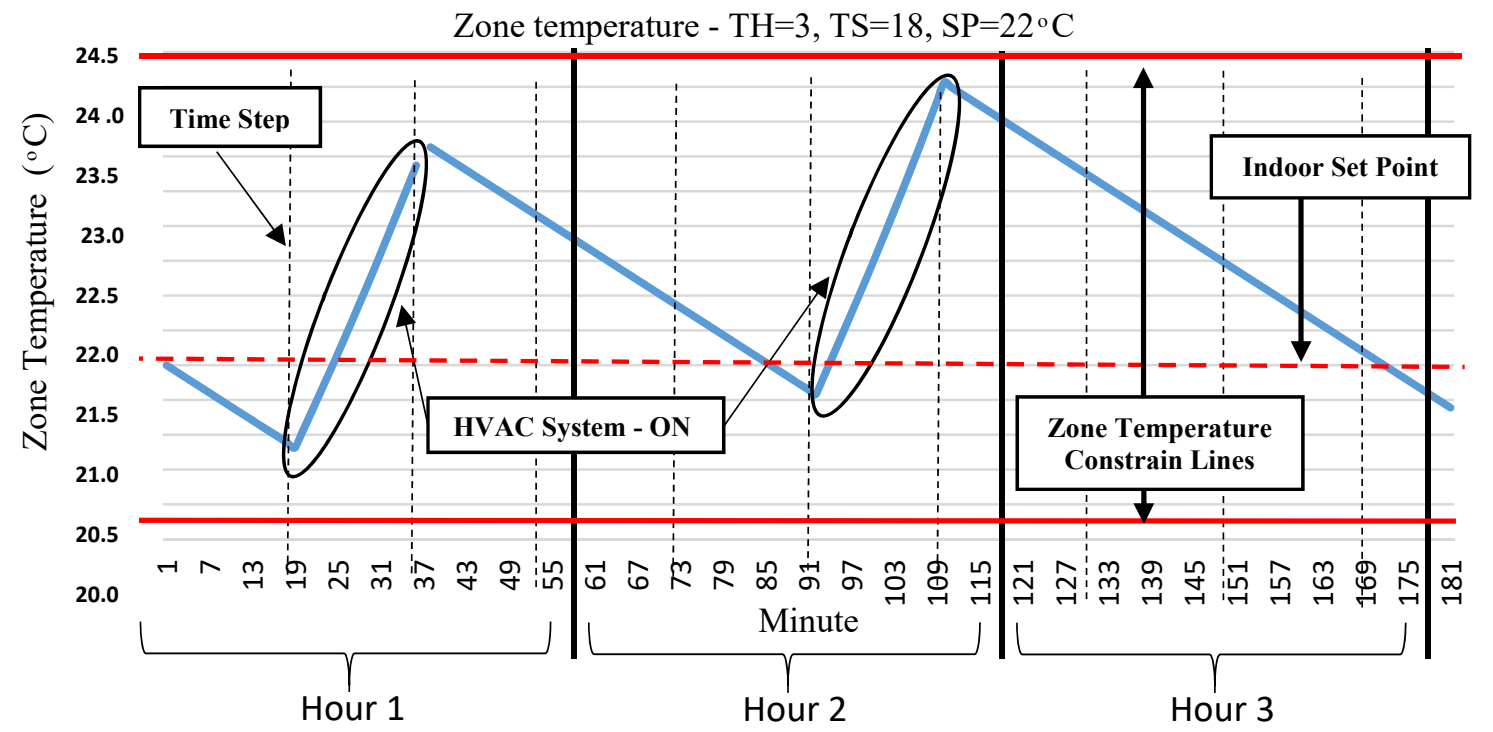

Figure 5-13: Zone temperature oscillation during 3 hours control time horizon 
By increasing the time step, the zone temperature oscillation (the deviation between the zone temperature and set point) increases. In other words, when a small time step is selected (i.e., 1minute) zone temperature precisely follows the indoor set point. However, in this condition, HVAC system turns on and off frequently, which could lead to inefficincy and shorter life expectancy of the HVAC system. Figure 5-14 demonstrates zone temperatures by choosing 24hour as the control time horizon, $22^{\circ} \mathrm{C}$ as a constant indoor set point, and 1-minute and 30-minute as the control time steps. As it is evident from Figure 5-14, the zone temperature deviation from the set point has significantly increased by selecting 30 -minute as the time step.

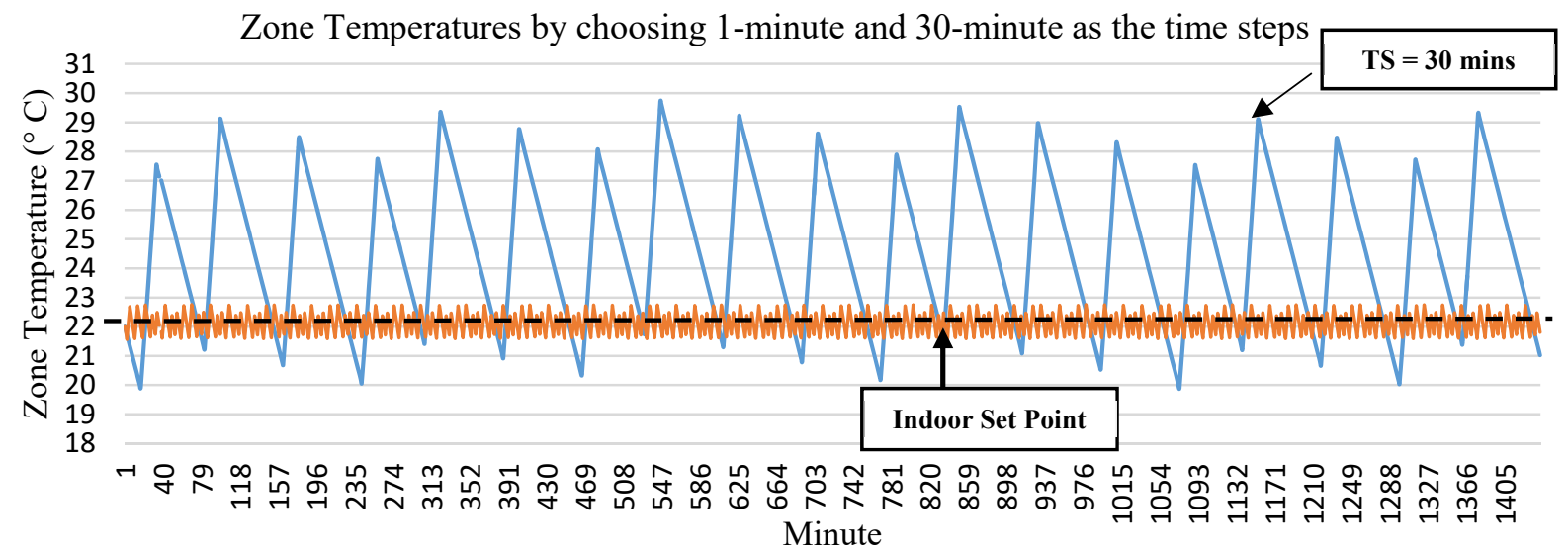

Figure 5-14: Zone temperatures by choosing 1-minute and 30-minute as the time steps

Figure 5-15 shows the switching times of the HVAC system for 1-minute and 30-minute time steps. As it is observed from Figure 5-15, in the 1-minute time step, HVAC system was frequently switched to follow the set point precisely.

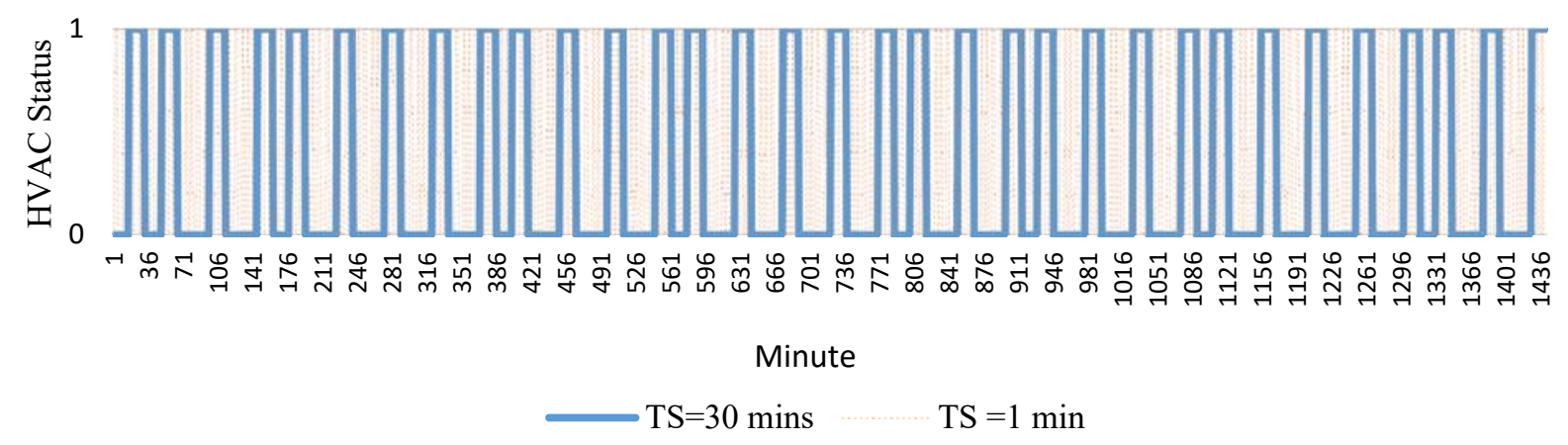

Figure 5-15: Switching times of HVAC system after selecting 1-minute and 30-minute time steps 
As mentioned before, one of the key tasks of the HVAC smart controller is to apply/implement the exhaustive optimization process to determine the optimum HVAC operation time step. In this optimization scheme, all described processes of first and second control loops are iterated using different control time steps. The objective of exhaustive optimization function, as the first optimization core (Core1), is to determine the optimum time step for each control time horizon and each hourly indoor set point profile in which the HVAC system energy demand (based on the time-dependent HVAC and house thermal models conditions) is minimum.

\subsection{Exhaustive Function Development}

Figure 5-16 demonstrates the framework/structure of the exhaustive optimization core (Core1) having a horizontal vector and two matrices. The horizontal vector shows the breakdown of HVAC system energy demand with 24-hour TH and 5-minute TS. Since 5-minute is selected as the time step, each hour consists of $12(60 / 5=12)$ cells in which the HVAC system energy demands in each time step $\left(\mathrm{D}_{\mathrm{i}}\right)$ are stored in $\mathrm{kW}$. A $\mathrm{kWh}$-convertor is designed in the exhaustive optimization core to convert the $\mathrm{Di}_{\mathrm{i}}$ into $\mathrm{kWh}$, and then records data in its corresponding cell in the left-side matrix. The left-side matrix in Figure 5-16 shows the HVAC system energy demand based on kWh during different time steps. In this test run, 1 and 30 minutes are respectively selected for minimum and maximum time steps when 24-hour is selected as the control time horizon. The dimensions of the left-side matrix can be changed when a different control time horizon and/or a different range of time steps are selected by the exhaustive optimization core or by the user.

The HVAC system energy demand, at the end of control time horizon, is calculated by adding all hourly HVAC system energy demand ( $\mathrm{kWh}$ ) for each particular time step. The HVAC system energy demands are then stored in the corresponding sum cells. Using this method, the left-side matrix is entirely transformed into a vertical vector that is stored in the $24^{\text {th }}$ column of the rightside matrix. In the next iteration, if 23 -hour were selected as the control time horizon, the generated vertical vector would be stored in the $23^{\text {rd }}$ column of the right-side matrix. The time step in which HVAC system energy demand is minimum (due to the time-basis condition) is simply selected by the exhaustive optimization core, comparing the elements of each vertical vector. It should be noted that the optimum time step maybe varied by changing the control time horizon and/or hourly indoor set point vector. 
One of the key parameters in the optimization process is the number of acceptable time steps, which ensures the zone temperature constraints. This number may be decreased by selecting a set point vector/pattern in which there is one or more set point(s) close to the zone temperature constraints.

Figure 5-17 shows the effect of set point vector on the number of acceptable time steps. In this simulation, the set point vector is prepared for the whole day when 24-hour is selected as the control time horizon. The set point vector is set to pre-heat the house by choosing $24^{\circ} \mathrm{C}$ as the indoor set point during off-peak hours and to reduce the HVAC energy demand during the peak hours by changing the indoor set point to $20^{\circ} \mathrm{C}$. In this simulation, $19^{\circ} \mathrm{C}$ and $27^{\circ} \mathrm{C}$ are selected as zone temperature constraints to let the HVAC smart controller run the HVAC system by a wider range of time steps.

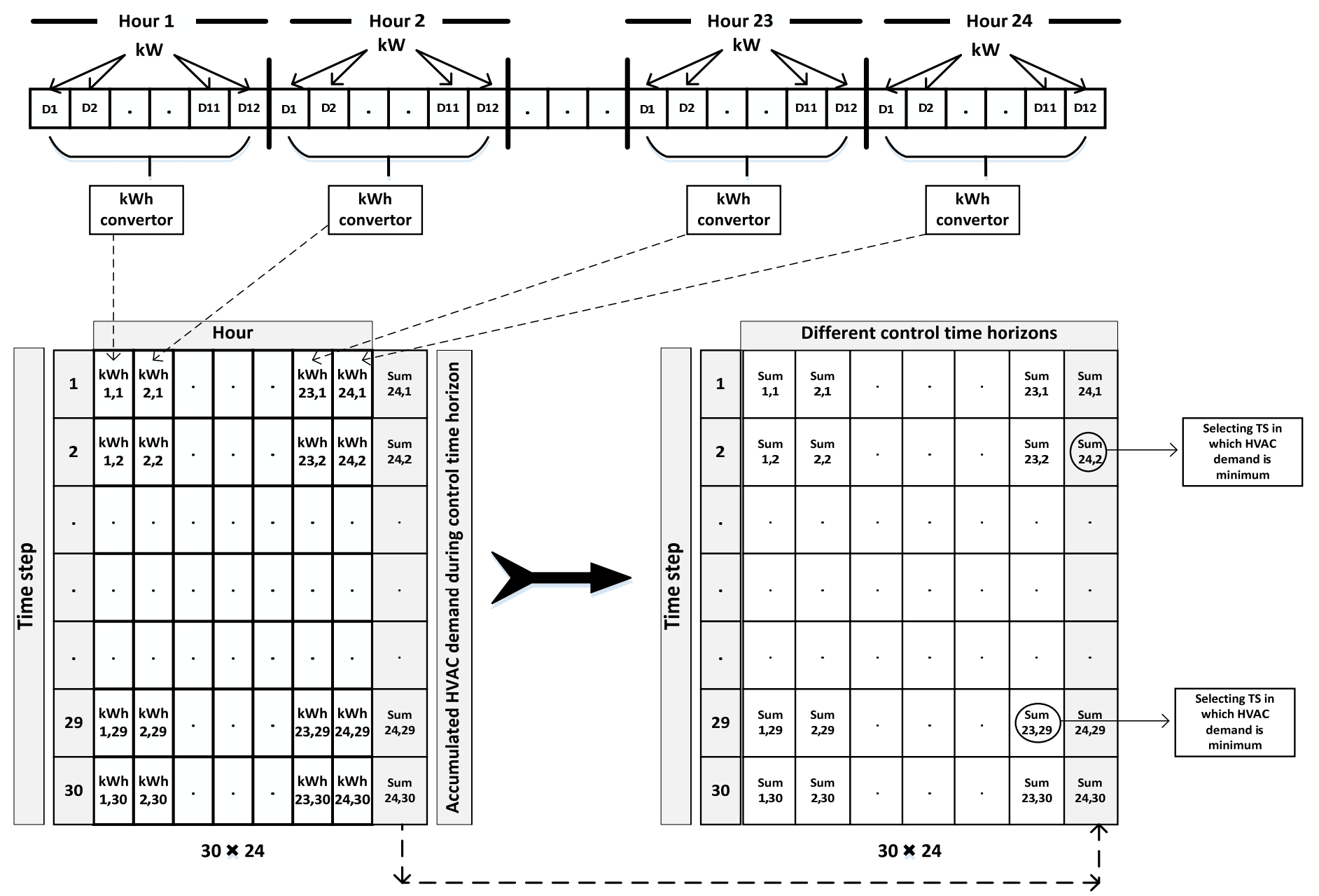

Figure 5-16: Framework/structure of the exhaustive optimization core 
However, after applying the exhaustive optimization, 12-minute TS was determined by the HVAC smart controller as the maximum allowed time step. This decision was made since selecting 13minute as the time step resulted in zone temperature violation by exceeding the temperature constraints. In this simulation, 6-minute is determined by the exhaustive optimization as the optimum time step. In a new test run, if $22^{\circ} \mathrm{C}$ were selected as the set point for the entire day, higher time step might be selected by the optimization core as the maximum allowed time step.

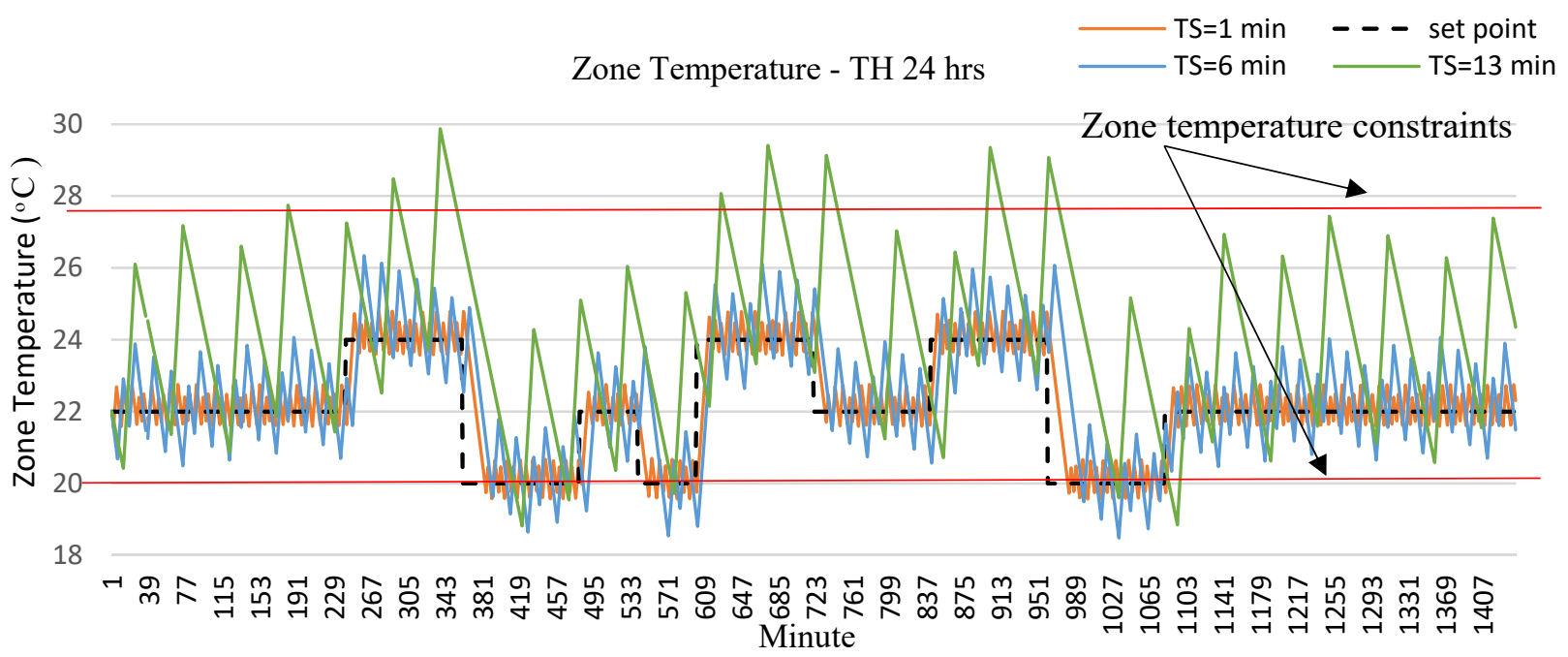

Figure 5-17: Result of an example test run simulation

In the HVAC smart controller, an hourly-based mechanism is developed which allows the user to set different zone temperature constraints for different hours of the day. This mechanism increases the number of acceptable time steps. As a result, the exhaustive optimization can offer more saving on the HVAC system energy demand by searching on a larger range of time steps.

Since TOU pricing scheme and supplied energy of renewable sources vary during different hours, the optimum time step in which HVAC system energy demand is minimum may not necessarily offer maximum saving in the HVAC system energy cost.

In the last step, the HVAC smart controller exports the optimization results to the next phase in Demand MPC controller for further post processing. The framework of HVAC smart controller function is illustrated in Figure 5-18. 


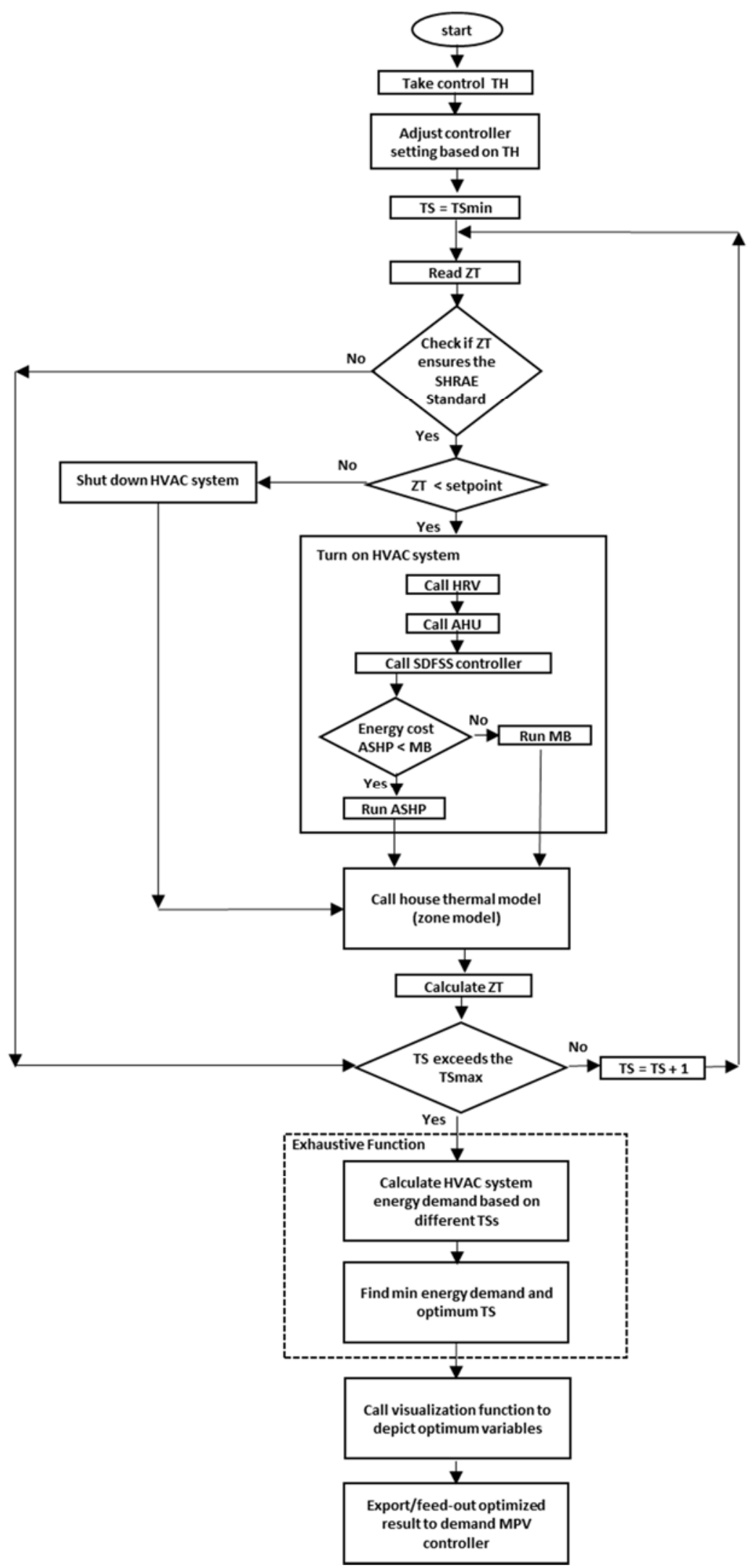

Figure 5-18: Framework of HVAC smart controller function 


\subsection{Arranged Indoor Set Point Generator (AISPG) Function}

Arranged Indoor Set Point Generator (AISPG) function is the second function applied to the Demand MPC controller. AISPG function consists of two sub-functions. In the first sub-function, called AISPG-OD, a smart decision support system (Core 2) is developed. AISPG-OD subfunction calculates and supplies a primary hourly indoor set point profile (called Arranged Indoor Set Point Vector) in which the advantages of outdoor disturbances (ODs) including outdoor temperature, solar irradiation, and wind speed are considered. This arranged indoor set point vector is set so that it enhances the HVAC system effectiveness/energy efficiency by taking the maximum advantage of outdoor disturbances. With this methodology, the optimization computational time is significantly decreased by directly selecting the most energy-efficient hourly indoor set point vector instead of running the optimization process considering the exhustive set point patterns in order to find the optimum one.

In the second sub-function, called AISPG-ME, the main principles of the developed decisionmaking method in AISPG-OD sub-function are utilized. AISPG-ME sub-function generates a new hourly indoor set point profile by taking maximum advantage of renewable as well as solar community's surplus energy sources. In this sub-function, the supplied energy of renewable and solar community's surplus energy sources are estimated for the future hours, and an hourly indoor set point vector is provided that takes maximum exploitation of these sources for supplying the HVAC system demand.

A methodology has been developed inside AISPG-OD and AISPG-ME sub-functions allowing these systems set their internal infrastructures/outputs based on the different control time horizons.

\subsection{AISPG-OD Sub-function}

Figure 5-19 shows the HVAC system demand pattern when the outdoor temperature changes between $-12^{\circ} \mathrm{C}$ and $8^{\circ} \mathrm{C}$ in a sample winter day. Based on Figure 5-19, regardless of the fact that HVAC system was ran based on a constant indoor set point $\left(22^{\circ} \mathrm{C}\right)$, the HVAC energy demand varied during different hours. This happens because of the effects of outdoor disturbances on the house thermal energy demand and subsequently HVAC system energy consumption. In this case, house thermal energy demand and subsequently HVAC system consumption increase during the hours in which outdoor temperature is colder, no external/internal gains are made from solar irradiation, and wind velocity is high. Conversely, HVAC energy consumption decreases when the 
outdoor temperature is warmer and/or external/internal gains are made by solar irradiation, and/or wind velocity is minimal. In other words, the effects of these outdoor disturbances create an oscillation in HVAC system demand even if a constant indoor set point is selected for the whole day. HVAC system energy demand during different hours of control time horizon demonstrates the consequence of combined outdoor disturbance effects on the house thermal demand.

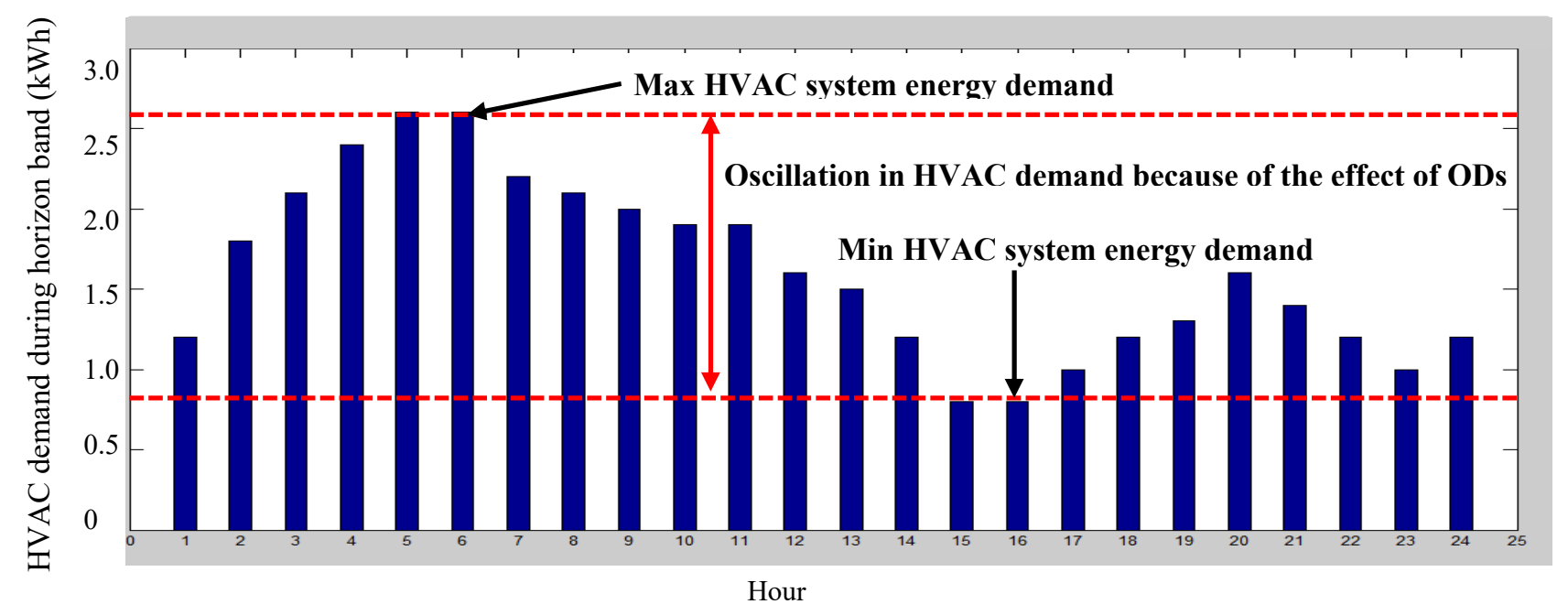

Figure 5-19: A sample of HVAC system optimum demand profile with 24hour control time horizon

The smart decision support system (SDSS), developed in this sub-function (Core 2), takes advantage of OD effects, so that it sets higher indoor set points for the hours in which HVAC system energy demand is low. In this method, a considerable thermal energy is stored inside the house during the hours in which the thermal energy gains from ODs are higher than the other hours. The advantages of this stored energy are utilized later in the hours in which the house thermal demand is higher due to less contribution of ODs. In contrast, lower indoor set points are set by the developed SDSS for the hours in which less thermal energy gains from ODs in order to reduce the HVAC system energy demand during these hours. A normalization method is utilized to employ the smart decision support system in the MPC model. In normalization method two cells, which represent the minimum and maximum demand, are selected from HVAC system energy demand vector. A normalized vector is generated based on these two cells and by using the following equation: 
Normalized HVAC Demand Vector $=\frac{\text { HVAC energy demand vector }- \text { Min demand }}{\text { Max demand }- \text { Min demand }}$

After running Equation (5-36), the arranged indoor set point vector, as the output of AISPG-OD sub-function, is generated based on the normalized vector as well as the user-defined minimum and maximum set points (MinSP and MaxSP, respectively) by using the following equation:

Arranged Indoor Set Point Vector $=$

$($ MinSP - MaxSP $) \times$ Normalized HVAC Demand Vector + MaxSP

It should be noted using Arranged Indoor Set Point Vector does not violate the thermal comfort since this vector is generated based on user-defined set point constraints.

Figure 5-20 shows the results of a test run of AISPG-OD sub-function when 24-hour is selected as the control time horizon and $20^{\circ} \mathrm{C}$ and $24^{\circ} \mathrm{C}$ are selected as MinSP and MaxSP, respectively.

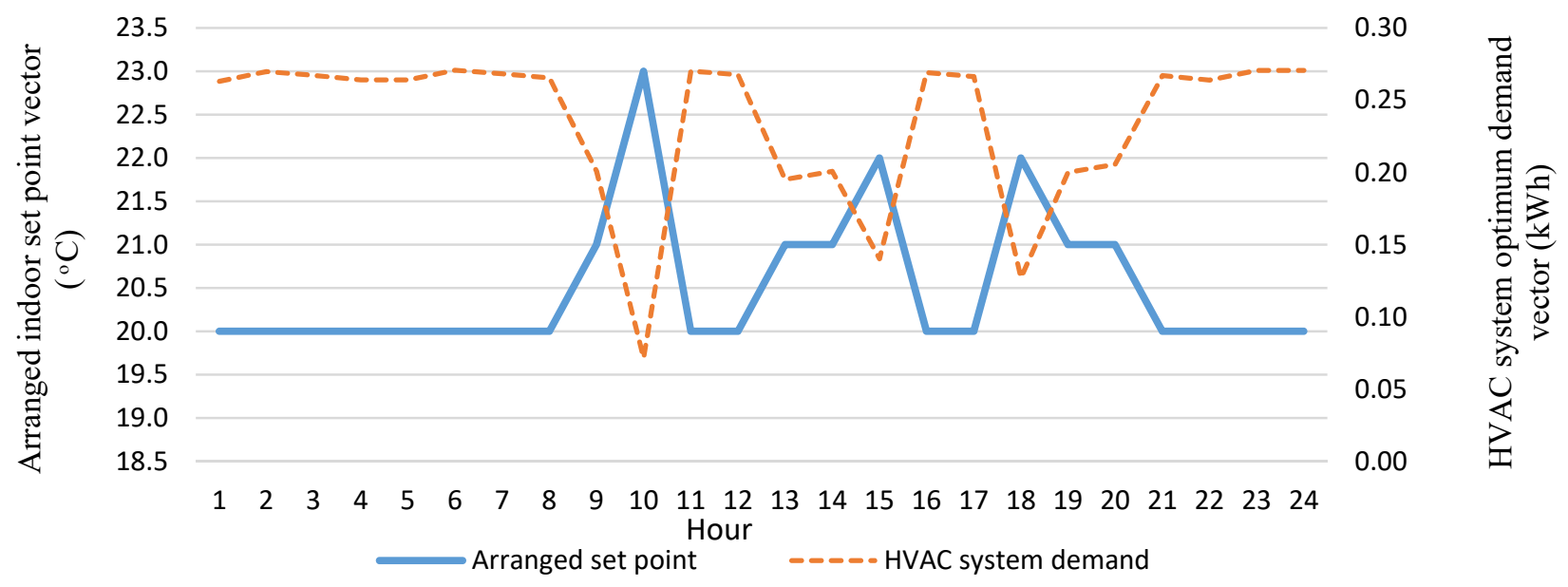

Figure 5-20: Arranged indoor set point vs. HVAC system demand

\subsection{AISPG-ME Sub-function}

This sub-function receives the supplied energy from renewable sources and the surplus energy of the solar community (which is forecasted based on FWD), user-defined minimum and maximum set points, and control time horizon as input (feed-in) variables. This sub-function returns the Arranged Indoor Set Point Vector as the output vector. The arranged indoor set point vector is supplied to maximize the usage of renewable and solar community energy sources, as the least 
expensive energy sources. To this end, higher indoor set points are proportionally selected for the hours in which the sum of aforementioned renewable sources is higher. In other words, this smart decision support system sets the HVAC system to utilize more energy from least expensive sources, convert it to thermal energy, and store the thermal energy. It then utilizes the stored thermal energy during the hours when these renewable sources are not available.

Like AISPG-OD sub-function, to employ the smart decision support system in MPC model, the following equations is used to take advantage of normalization method:

Integrated supplied power $=$ PV supplied power + WT supplied power + solar community supplied power

Normalized Integrated Supplied Power $=\frac{\text { Integrated supplied power }}{\text { Max (Integrated supplied power })}$

Arranged Indoor Set Point Vector $=$

$$
\text { MinSP + (Normalized Integrated Supplied Power } \times(\text { MaxSP }- \text { MinSP }))
$$

Since this mechanism utilizes maximum exploitation of renewable energies, excellent saving on the HVAC system energy cost can be achieved without sacrificing indoor thermal comfort.

At the end of AISPG function, two horizon-based arranged indoor set point vectors are generated by AISPG-OD and AISPG-ME sub-functions while considering the optimum time step, outdoor disturbances, and the availability of renewable and solar community sources. In the next function of Demand MPC controller (called Comprehensive Set Point Matrix Generator (CSPMG) function), a matrix containing new indoor set point vectors would be generated based on the supplied arranged indoor set point vectors. This matrix incorporates more energy cost saving value into the arranged indoor set point vectors by considering the effects of TOU pricing scheme as one of the pricing factors.

\subsection{Comprehensive Set Point Matrix Generator (CSPMG) Function}

The savings offered by the arranged indoor set point vectors will be significantly increased by employing CSPMG function as the second smart decision support system (Core 3). This function arranges indoor set points during different hours so that it sets higher set points during off-peak 
hours to pre-heat the house while setting lower set points during mid-peak/peak hours to reduce the HVAC system energy cost during these periods when electricity is more expensive. In the entire set point arrangement/tuning process, the user-defined zone temperatures are considered as the constraints to avoid thermal comfort violation. Since different indoor set points within userdefined limits can be set during off-, mid- and peak hours, a mechanism has been developed to generate all possible set point patterns/profiles (states) that result in pre-heating the house during off-peak hours as well as HVAC system energy demand reduction during mid-peak/peak hours. In other words, with this mechanism, from the very broad possible set point patterns only useful set point patterns that lead to saving on the HVAC system energy cost are generated. Utilizing this mechanism decreases the number of set point patterns and iterations that hence, reduces the optimization computational time. This mechanism is capable of adapting itself with various control time horizons, which are determined/set later by the Demand MPC controller. Figure 5-21 shows off-, mid-, and peak hours in the winter season, in Ontario, Canada. As Figure 5-21 shows, three different switching points have been considered between TOU prices in CSPMG function. As described before, CSPMG function generates useful set point patterns that behave like energy conservation strategy planning models for two hours before and after each switching point.

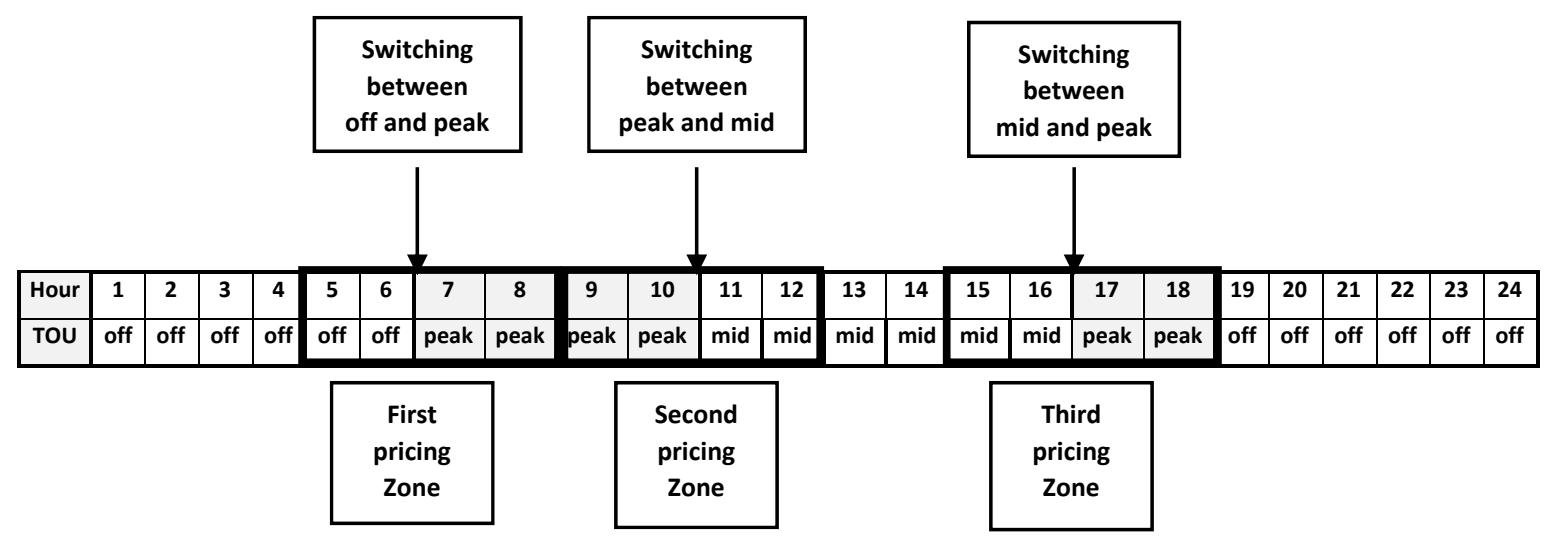

Figure 5-21: Three different TOU price switching points in winter season in Ontario

Figure 5-22 shows a test run example. In this test run, the set points inside the first zone hours are set based on the arranged indoor set point vectors. In this test run, $20^{\circ} \mathrm{C}$ and $24^{\circ} \mathrm{C}$ are selected by the user as the indoor set point constraints. Since set point at 6 am is $24^{\circ} \mathrm{C}$ (which set by AISPG function), there is no degree of freedom at this particular hour for pre-heating the house by 
rearranging/increasing the set point (selecting higher set points exceeds the user-defined $24^{\circ} \mathrm{C}$ constraint). Similarly, there is no degree of freedom for decreasing the HVAC demand by falling down the set point at 8 am as a peak hour (selecting lower set points exceeds the user-defined $20^{\circ} \mathrm{C}$ constraint). However, the set point at 5 am has two degrees of freedom for increasing the set point to pre-heat the house. Similarly, the set point at 7 am also has two degrees of freedom for lowering the set point in order to decrease the HVAC demand in this peak hour.

\begin{tabular}{|c|c|c|c|c|}
\hline & Off-peak & Off-peak & peak & peak \\
\hline Hour & 5 & 6 & 7 & 8 \\
\hline SP & 22 & 24 & 22 & 20 \\
\hline & $\begin{array}{l}2 \text { freedom } \\
\text { degrees up }\end{array}$ & 0 & $\begin{array}{c}2 \text { freedom } \\
\text { degrees down }\end{array}$ & 0 \\
\hline
\end{tabular}

Figure 5-22: Degrees of freedom in first zone hours

After determining the degrees of freedom for the four hours of the first pricing zone, a mechanism is developed in the second sub-section of CSPMG function using a probability technique to generate a matrix containing all possible useful indoor set point patterns/profiles that lead to energy cost conservation. Based on the concept of the developed mechanism, all generated set point patterns have the potential to offer saving on the HVAC system energy cost.

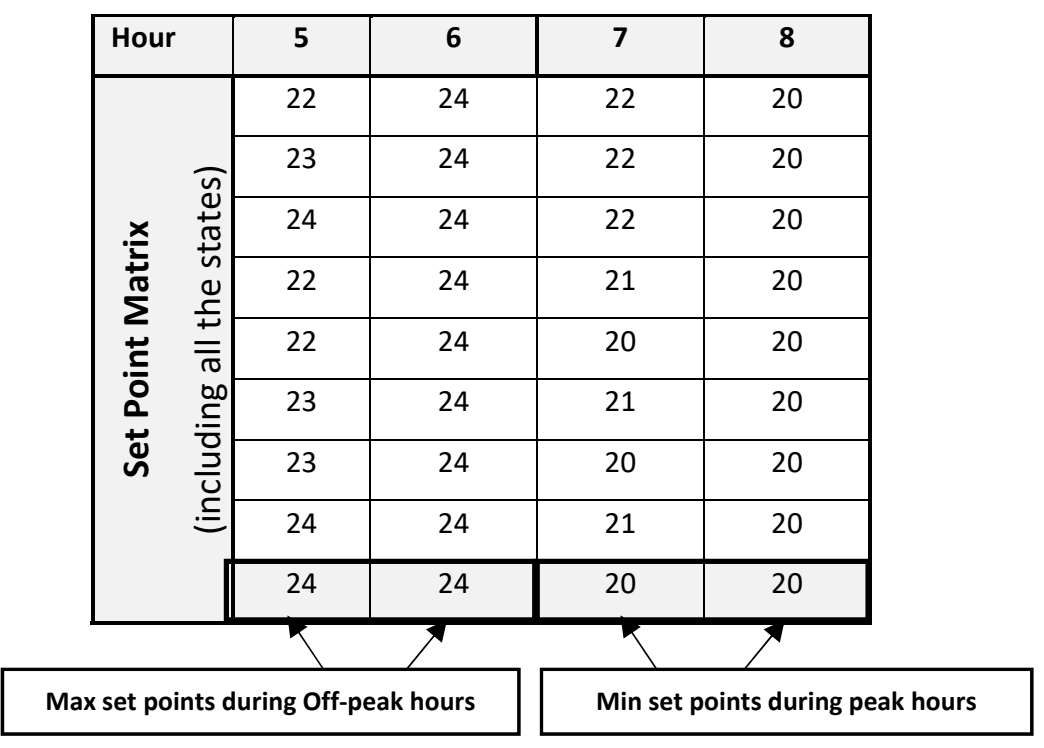

Figure 5-23: Generated set point patterns matrix containing nine states 
For example, if $20^{\circ} \mathrm{C}$ is set for 5 am and 6 am and $24^{\circ} \mathrm{C}$ is set for 7 am and 8 am as the set points (while $20^{\circ} \mathrm{C}$ and $24^{\circ} \mathrm{C}$ are selected by the user as the minimum and maximum indoor set point constraints, respectively), each hour would have four degrees of freedom. As a result, the generated matrix should have $256\left(4^{4}\right)$ states. To this end, a complex probability problem is solved in the second sub-section to generate all the possible states. Using the information presented in Figure 522, a matrix containing nine states can be generated as shown in Figure 5-23.

The second and third pricing zones use the same methodologies employed in the first pricing zone for generating a matrix of useful indoor set point patterns. As the last step of CSPMG function, three indoor set point pattern matrices that were supplied in the first, second, and third pricing zones are combined together to generate a comprehensive indoor set point pattern matrix that covers the 24 hours of a day.

If it is assumed that there is four degrees of freedom for each hour in all pricing zones (in other words, if each pre-generated indoor set point pattern matrix has 256 states), the comprehensive matrix would have more than 16 million states $\left(256^{3}\right)$. However, this situation would never happen in practice since the rate of outdoor temperature change is not fast enough to allow the AISPG function to generate peak indoor set points for all three pricing zones' hours. Even if we consider this impossible hypothesis, CSPMG function will drastically reduce the possible conditions of different set point patterns $\left(4^{24} \cong 28 * 10^{13}\right)$ which can be considered for the entire day. As a result, this assessment proves the effectiveness of CSPMG function in decreasing the computational time.

A test run has been performed to show the effect of each indoor set point profile (inside the comprehensive matrix) on the HVAC system behavior. To this end, the CSPMG function is run considering 24 -hour, $20^{\circ} \mathrm{C}$ and $24^{\circ} \mathrm{C}$ as the control time horizon, and user-defined minimum and maximum indoor set point constraints, respectively. The user-defined minimum and maximum zone temperature constraints are $18^{\circ} \mathrm{C}$ and $26^{\circ} \mathrm{C}$, respectively. In this test run, a comprehensive matrix was generated by CSPMG function containing 12,290 states. 
Figure 5-24 shows the first indoor set point pattern (the first state of the comprehensive matrix) compared to the arranged indoor set point pattern generated by AISPG function. As Figure 5-24 shows, one unit growth and one unit decrement took place in indoor set points respectively in all off-peak and peak hours having the degree(s) of freedom. The set point remained the same in the off-peak and peak hours with no degree of freedom.

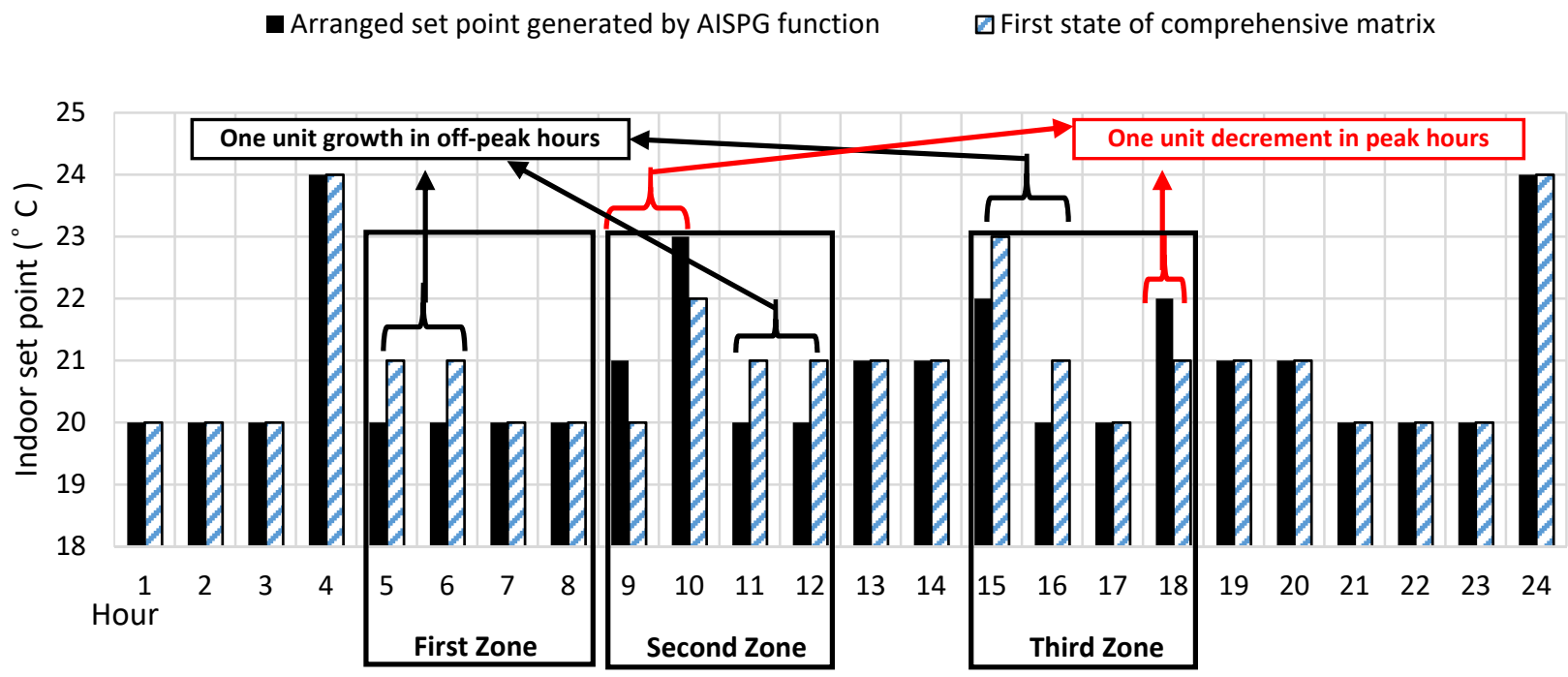

Figure 5-24: Rearranging the indoor set points in the first state of comprehensive matrix

Figure 5-25 demonstrates hourly HVAC system energy demand using arranged set point pattern and the first state of comprehensive matrix. It should be noted that both HVAC system energy demands are optimal in terms of HVAC operating time step since these demand vectors are the output of House-HVAC integrated function utilizing exhaustive search optimization.

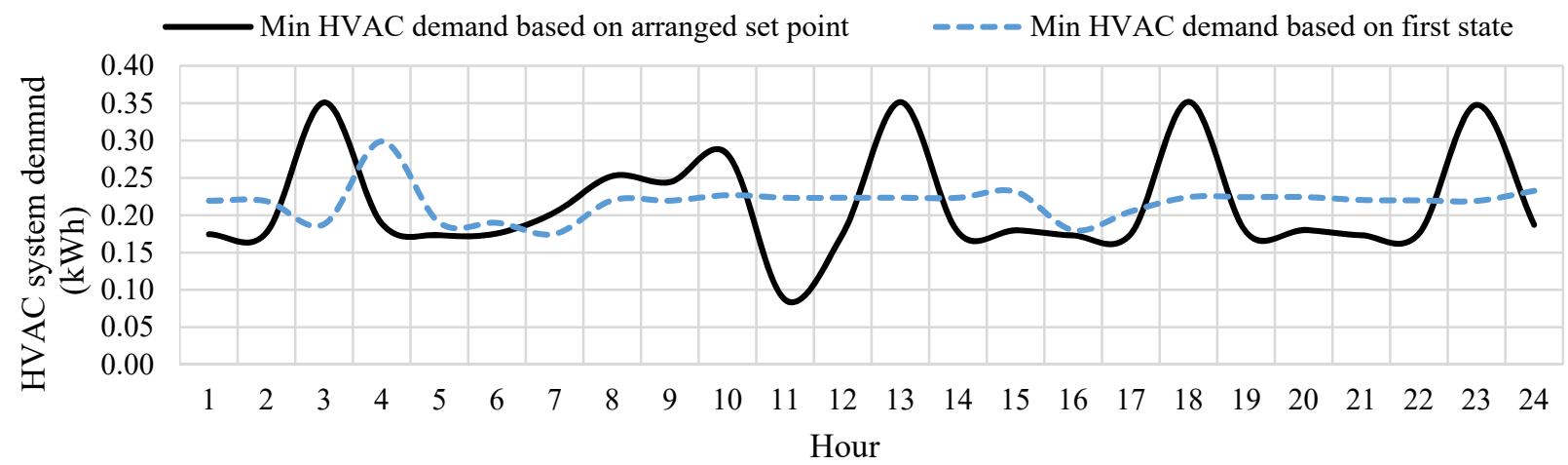

Figure 5-25: HVAC system energy demand using arranged and first state of comprehensive matrix set point patterns 
Figure 5-26 illustrates the zone temperature for the entire day under the effects of the last state indoor set point pattern. This figure perfectly shows the pre-heating during off-/mid-peak hours and zone temperature reduction during peak hours. Furthermore, the advantages of the arranged set point pattern were previously taken during the hours that are not included in the three pricing zones.

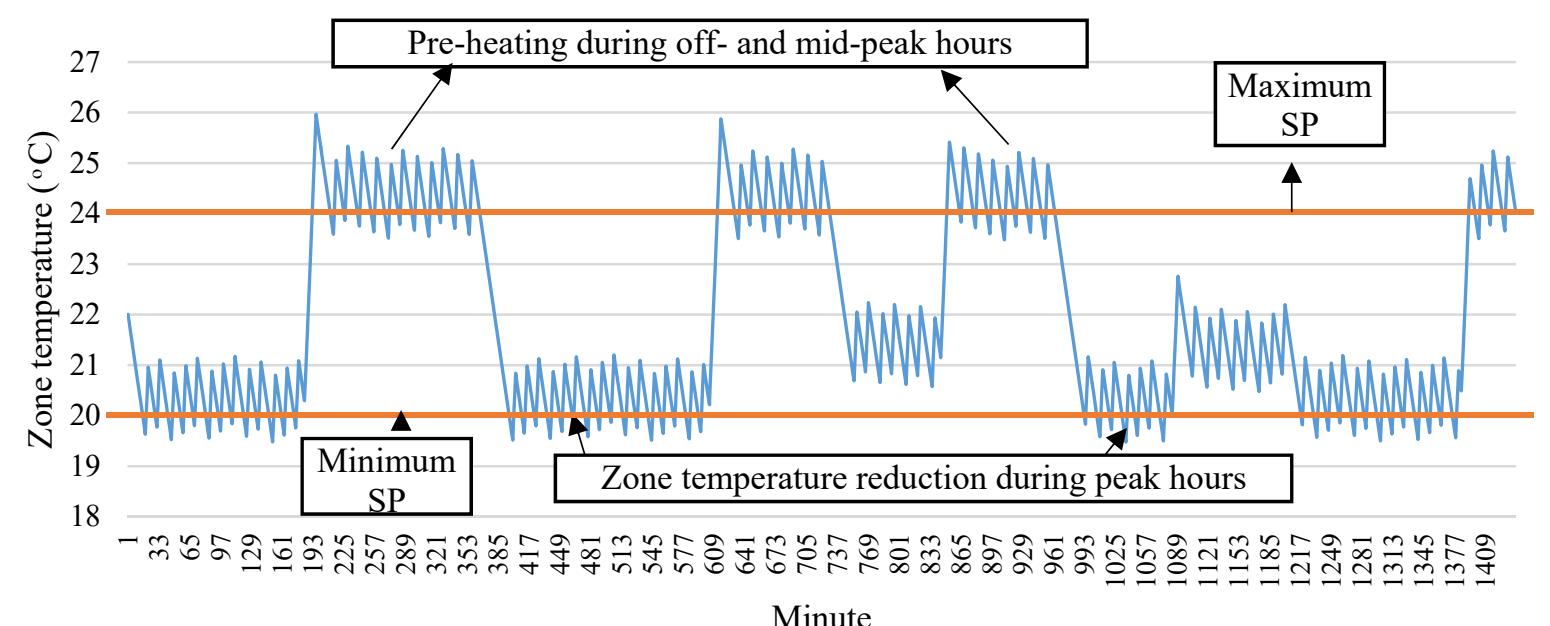

Figure 5-26: Zone temperature in entire day under the effects of last state indoor set point pattern

Regardless of the fact that all supplied indoor set point profiles inside the comprehensive matrix offer saving on the HVAC system energy cost, the savings that presented by some of them are higher due to the effects of outdoor disturbances and availability of renewable energy sources. As a result, a linear optimization core (the last core (Core 4) of the Demand MPC optimization) is designed to determine the state/indoor set point pattern that offers maximum saving on the HVAC system energy cost considering the effects of outdoor disturbances, renewable supplied power, solar community surplus energy, storage system and so on.

\subsection{Linear Optimization Function}

Linear optimization function is the last core of optimization (Core 4) of the Demand MPC controller. In this core, the final optimum set point pattern, which generates minimum HVAC system energy cost, is determined. A mechanism is developed in this function to adapt 
optimization processes and matrices/vectors dimensions based on the control time horizon. The costs of energy generated by the energy sources (including renewables, solar community, storage system and utility (TOU)) have been considered as decision making weighting factors in the linear optimization objective function. Figure 5-27 shows the input and output variables of the linear optimization function.

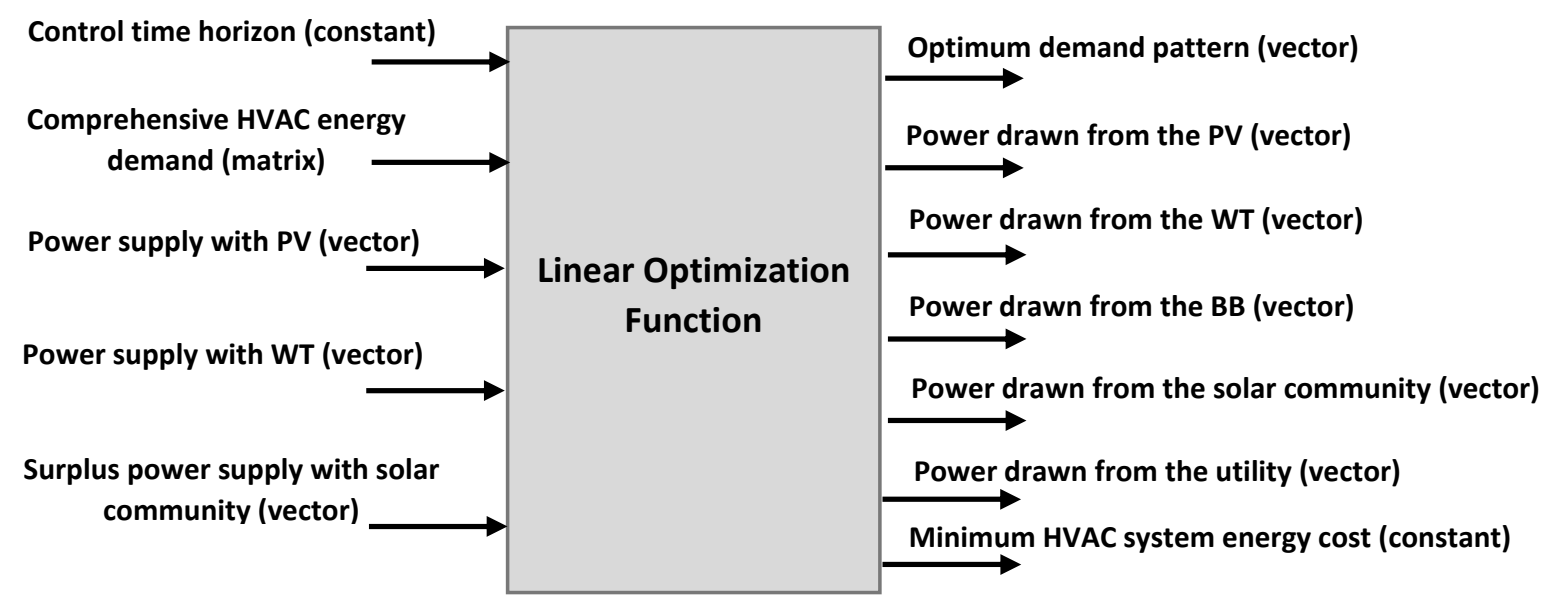

Figure 5-27: Input and output variables of the linear optimization function

Equation (5-39) shows the construction of a linear optimization program while $x$ is a vector consisting the decision making variables in the objective function; $A$ and $A_{e q}$ are matrices containing the coefficients of decision making variables in inequality and equality equations, respectively; $b$ and $b_{e q}$ are vectors containing the constraints of inequality and equality equations, respectively; and $l b$ and $u b$ indicate the lower and upper bounds of the decision making variables, respectively.

$$
\mathrm{f}(\mathrm{x}) \text { so that }\left\{\begin{array}{l}
A \times x \leq b \\
A_{e q} \times x \leq b_{e q} \\
l b \leq x \leq u b
\end{array}\right.
$$

"linprog", a Matlab built-in library function, is used for constructing the optimization function. Equation (5-40) illustrates the input and output variables of "linprog" function where $w$ is a vector 
containing the weighting factors of decision-making variables into the objective function and DMVariables_value and Fval return the value of the decision-making variables and the objective function value, respectively.

[DMVariables_value, Fval] $=\operatorname{linprog}\left(W, A, b, A_{e q}, b_{e q}, I b, u b\right)$

\subsection{Constructing Linear Optimization Based on the Process Model Configuration}

As mentioned before, five different energy sources, including PV, WT, solar community surplus energy, battery bank storage system, and utility/local grid supply the HVAC system energy demand during the control time horizon. Each energy source has its own energy cost. As a result, in each hour, HVAC system energy cost is calculated based on the contribution and subsequently the costs of these energy sources in supplying the HVAC system energy demand. In this case, the objective function of linear optimization is presented by the following equation:

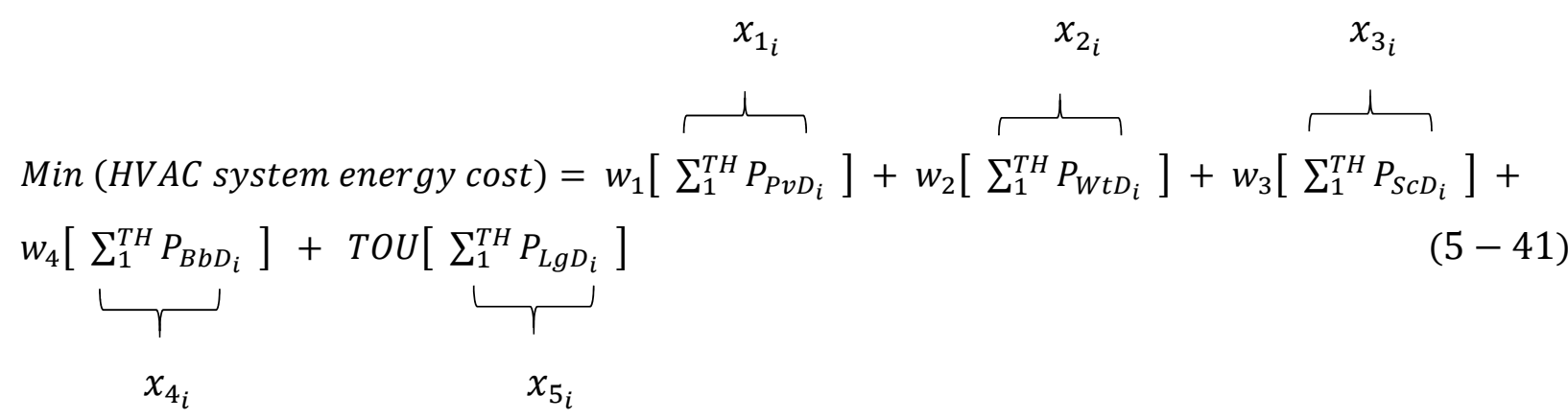

In Equation (5-41), $P_{P v D_{i}}, P_{W t D_{i}}, P_{S c D_{i}}, P_{B b D_{i}}$, and $P_{L g D_{i}}$ indicate the energy (kWh) drawn from PV, WT, solar community, battery bank, and local grid, respectively, to meet the HVAC system demand $\left(D_{i}\right)$ in hour i. TH indicates control time horizon and TOU represents the time of use pricing scheme. $w_{1}, w_{2}, w_{3}, w_{4}$, and TOU show the weighting factors (WF) of PV, WT, solar community, battery bank, and local grid energy sources, respectively. All weighting factors indicated by $w$ index are constant in the entire control time horizon; TOU however varies with time. $w_{i}$ weighting factors are calculated based on the capacity cost investment of equipment. For example, based on U.S. Photovoltaic Prices and Cost Breakdowns [5.53], PV energy cost is estimated as $\$ 4.5 / \mathrm{kW}(\mathrm{CAD} \$)$. Also, based on the WT cost calculations presented in [5.54], WT energy cost is estimated as $\$ 6 / \mathrm{kW}(\mathrm{CAD} \$)$. However, since additional maintenance, transmission, distribution and protection costs are considered for the energy provided by solar community, the 
solar community energy cost is estimated as $\$ 7.5 / \mathrm{kW}$ (CAD\$) in this thesis by considering $30 \%$ overhead on the average energy cost of PV and WT. Based on the fact that battery bank systems are expensive (have high capital cost) as well as need high maintenance requirement, $\$ 15.5 / \mathrm{kW}$ (CAD\$) is considered as the battery bank system energy cost to minimize the usage/size of battery bank systems (battery bank only use during mid- and peak-hours). Table 5-5, illustrates the weighting factor vector defined in this optimization problem. These weighting factors indicate the rated costs of energy sources.

Table 5-5: Optimization problem-weighting factor vector

\begin{tabular}{|c|c|c|c|c|}
\hline Energy Source & PV & WT & SC & BB \\
\hline WF & $W_{1}$ & $W_{2}$ & $W_{3}$ & $W_{4}$ \\
\hline Value & 4.5 & 6 & 7.5 & 15.5 \\
\hline
\end{tabular}

Since the objective function is minimized by linear optimization, the priority for supplying HVAC system demand is given to renewable and solar community energy sources that have lower WFs. Based on this WF vector, the first energy source, which is used by the optimization problem to supply the HVAC system demand, is PV. Afterwards, the HVAC system's unmet demand is covered by WT and then by solar community. Using this methodology, optimization problem encourages the usage of clean and sustainable renewable sources for supplying the HVAC system demand, which is one of the significant goals of designing NZEBs. Since TOU changes between $13.30 \phi / \mathrm{kWh}, 17.80 \phi / \mathrm{kWh}$ and $22.60 \phi / \mathrm{kWh}$ during off-peak, mid-peak and peak hours respectively; battery bank system can only be used during mid- and peak load hours. In other words, based on high capital and operation as well as maintenance costs of the battery bank system, the optimization problem is set to use local grid during off-peak hours instead of using battery bank system, reducing the usage of battery bank system.

Figure 5-28 shows the energy flow management diagram of the case study house. 


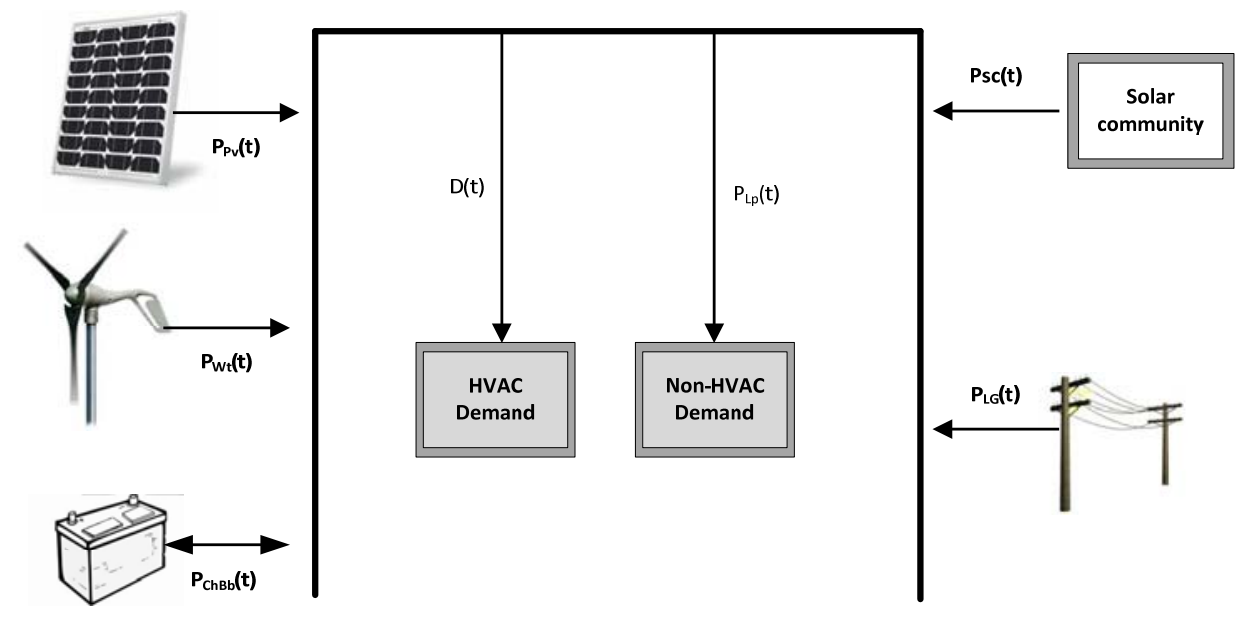

Figure 5-28: Energy flow management diagram of the case study house

The following set of equations, which are defined based on Figure 5-28, model the HVAC system energy balance during the control time horizon.

$$
\begin{gathered}
P_{P v D_{1}}+P_{W t D_{1}}+P_{S c D_{1}}+P_{B b D_{1}}+P_{L g D_{1}}=D_{1} \\
P_{P v D_{2}}+P_{W t D_{2}}+P_{S c D_{2}}+P_{B b D_{2}}+P_{L g D_{2}}=D_{2} \\
\cdot \\
\cdot \\
\cdot \\
P_{P v D_{T H-1}}+P_{W t D_{T H-1}}+P_{S c D_{T H-1}}+P_{B b D_{T H-1}}+P_{L g D_{T H-1}}=D_{T H-1} \\
P_{P v D_{T H}}+P_{W t D_{T H}}+P_{S C D_{T H}}+P_{B b D_{T H}}+P_{L g D_{T H}}=D_{T H}
\end{gathered}
$$

In certain hours in which solar radiation is stronger and more intensive (i.e., middle of the day) and/or wind velocity is high, available renewable sources may exceed the house load demand. As a result, during these hours the surplus energy is utilized to charge $\left(P_{C h B b_{i}}\right)$ the battery bank system. Based on the defined weighting factor vector, this surplus energy would be used later during midand/or peak hours to supply the HVAC system demand. The following set of equations shows the electricity flow management during different hours of control time horizon. 


$$
\begin{gathered}
P_{P v D_{1}}+P_{W t D_{1}}+P_{S c D_{1}}+P_{B b D_{1}}+P_{L g D_{1}}-D_{1}-P_{L p_{1}}=P_{C h B b_{1}} \\
P_{P v D_{2}}+P_{W t D_{2}}+P_{S c D_{2}}+P_{B b D_{2}}+P_{L g D_{2}}-D_{2}-P_{L p_{2}}=P_{C h B b_{2}} \\
\cdot \\
\cdot \\
\cdot \\
P_{P v D_{T H-1}}+P_{W t D_{T H-1}}+P_{S c D_{T H-1}}+P_{B b D_{T H-1}}+P_{L g D_{T H-1}}-D_{T H-1}-P_{L p_{T H-1}}=P_{C h B b_{T H-1}} \\
P_{P v D_{T H}}+P_{W t D_{T H}}+P_{S c D_{T H}}+P_{B b D_{T H}}+P_{L g D_{T H}}-D_{T H}-P_{L p_{T H}}=P_{C h B b_{T H}}
\end{gathered}
$$

In Equation (5-43), $P_{L p_{i}}$ indicates the demand of non-HVAC house loads (including appliances, lighting, and so on) in hour i, which is available from the house load profile file as external input. Since the state of charge (SOC) of battery bank system in hour i depends on the stored energy in previous hours and the rate of charging/discharging in hour $\mathrm{i}$, the following equations are used to model the behaviour of the battery bank system during different hours of control time horizon.

$$
\begin{gathered}
P_{B b-M I N} \leq P_{B b D_{1}} \leq P_{B b-\text { Initial }} \\
P_{B b-M I N} \leq P_{B b D_{2}} \leq P_{B b-\text { Initial }}+P_{C h B b_{1}} \\
P_{B b-M I N} \leq P_{B b D_{3}} \leq P_{B b-\text { Initial }}+P_{C h B b_{1}}+P_{C h B b_{2}} \\
\cdot \\
\cdot \\
\cdot \\
P_{B b-M I N} \leq P_{B b D_{T H}} \leq P_{B b-\text { Initial }}+\sum_{1}^{T H-1} P_{C h B b_{i}}
\end{gathered}
$$

In Equation (5-44), $P_{B b-\text { Initial }}$ indicates the initial SOC of the battery bank system. Thus, in the first running hour $(\mathrm{i}=1)$, the power drawn from the battery bank system should be limited to the initial state of charges of the battery bank system. However, to increase the lifetime of battery bank, the SOC of battery bank system should be kept higher than the minimum SOC $\left(P_{B b-M I N}\right)$ 
defined by the manufacturer. It should be noted that $P_{C h B b_{i}}$ can be positive or negative depending on the values of load demand and surplus energies in each hour.

In order to keep the optimization problem stable, all the boundaries of decision-making variables should be limited to reasonable ranges, which are defined based on the charactristics of the process model. To this end, the following equations are defined to limit the supplied power of energy sources as the decision-making variables:

$0 \leq P_{P v D_{i}} \leq P_{P V-M a x}$

$0 \leq P_{W t D_{i}} \leq P_{W T-\operatorname{Max}}$

$0 \leq P_{S c D_{i}} \leq P_{S C-\operatorname{Max}}$

$0 \leq P_{L g D_{i}} \leq P_{L G-M a x}$

$P_{P V-M a x}$, and $P_{W T-M a x}$ indicate the maximum capacity of PV and WT, respectively, and are set based on the characteristic of installed equipment. $P_{S c-M a x}$ is the maximum surplus power determined based on the solar community limitation. However, in this chapter, it is assumed that the solar community is capable to cover the maximum hourly demand of the house. $P_{L g-\operatorname{Max}}$ is also set based on the maximum hourly demand of the house. As mentioned before, Demand MPC controller covers the house unmet loads using utility/ local grid as a secure uninterruptable source. In each iteration of the optimization process, $b_{e q}$ vector, which contains HVAC hourly energy demand for all hours in the control time horizon, is defined based on the corresponding line/row of the comprehensive HVAC energy demand matrix. In other words, the linear optimization procedure is performed for each line of the comprehensive HVAC energy demand matrix. At the end of the linear optimization procedure, the objective function value, which shows the minimum HVAC system energy cost (after taking advantage of all energy sources), is stored in Fval vector.

Comparing the Fval vector elements, the minimum number/element, and subsequently the HVAC hourly demand pattern that generated the minimum number/element are determined as the final optimum solutions to be exported to the Demand MPC controller for further post-processing. Since each HVAC system hourly demand pattern is generated based on an hourly set point profile, Demand MPC controller determines the optimum hourly set point profile resulting in the minimum energy cost based on temporal outdoor disturbance. After selecting the optimum set point pattern, 
the powers that have been drawn from energy sources using this optimum set point pattern are read from DMVariables_value matrix and then exported to the Demand MPC controller.

After finishing all the optimization cores (Cores 1 to 4), the following optimum vector (Figure 529) is generated and presented by the Demand MPC controller by collecting all the optimum results. The HVAC system performance would then be conducted based on this optimum vector.

\begin{tabular}{|c|c|c|c|c|c|}
\hline $\begin{array}{c}\text { Optimum TH } \\
\text { (energy cost) }\end{array}$ & $\begin{array}{c}\text { Optimum TS } \\
\text { (energy cost) }\end{array}$ & $\begin{array}{c}\text { Minimum } \\
\text { energy cost }\end{array}$ & $\begin{array}{c}\text { Optimum TH } \\
\text { (energy demand) }\end{array}$ & $\begin{array}{c}\text { Optimum TS } \\
\text { (energy demand) }\end{array}$ & $\begin{array}{c}\text { Minimum } \\
\text { energy demand }\end{array}$ \\
\hline
\end{tabular}

Figure 5-29: Collecting all the optimum results to prepare the optimum vector

\subsection{Simulation Result}

Two different scenarios have been selected for employing the Demand MPC controller, analyzing its performance, and comparing its effectiveness versus conventional on-off and simple rule-based (SRB) controllers. In the first scenario, a typical winter day is selected. In this sample day, first, HVAC system energy cost is calculated for the whole day when a conventional on-off controller is used for regulating the zone temperature around $22^{\circ} \mathrm{C}$ as a constant indoor set point. In the next step, Demand MPC controller is employed on the system and HVAC system energy cost is compared with that of the conventional on-off controller.

In the second scenario, the Demand MPC controller is employed in the heating season. In order to measure the effectiveness of MPC controller, three base case scenarios (using conventional on-off controllers) and one simple rule-based controller have been used. In the first base case scenario, $20^{\circ} \mathrm{C}$ was selected as the indoor set point for the entire day while $22^{\circ} \mathrm{C}$ and $24^{\circ} \mathrm{C}$ were selected as the constant indoor set points in the second and third base case scenarios, respectively. HVAC system energy cost is calculated after running each base case scenario. In order to investigate the effectiveness of Demand MPC controller versus an efficient HVAC controller, a simple rule-based controller is used. SRB controller sets the indoor set point profile based on the TOU pricing scheme. In other words, $20^{\circ} \mathrm{C}$ is selected by SRB controller as the lowest ASHRAE Comfort Standard temperature for the peak hours while $22^{\circ} \mathrm{C}$ and $24^{\circ} \mathrm{C}$ (highest ASHRAE Comfort Standard temperature) are selected as the indoor set points during mid- and off-peak hours, 
respectively. Since lower set points are chosen in SRB controller for mid- and peak hours (when electricity is expensive), this controller itself results in saving in the HVAC system energy cost.

\subsubsection{Simulation Result for a Typical Winter Sample Day}

January $4^{\text {th }} 2015$ is selected as a typical winter sample day. Figure 5-30 shows the solar irradiation on different orientations (azimuth angles) of the house in this day.

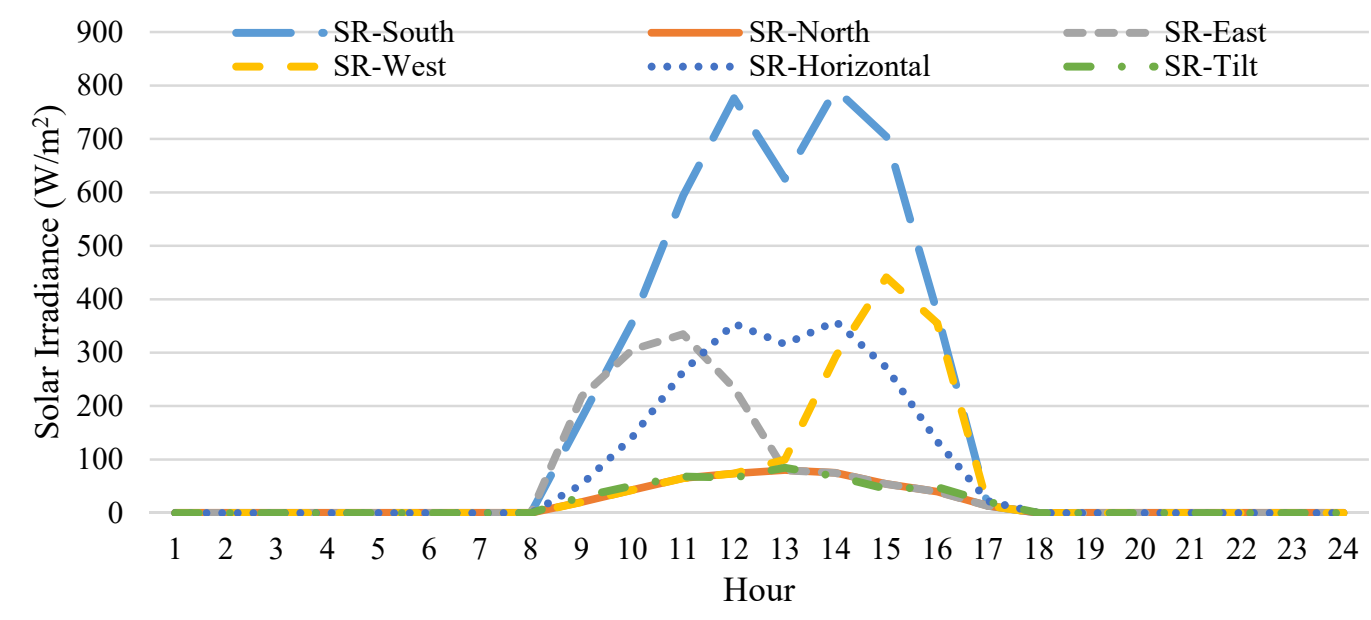

Figure 5-30: Solar irradiation on different orientations of the case study house

Figure 5-31 depicts outdoor temperature $\left({ }^{\circ} \mathrm{C}\right)$ and wind speed $(\mathrm{m} / \mathrm{s})$ in the sample day.

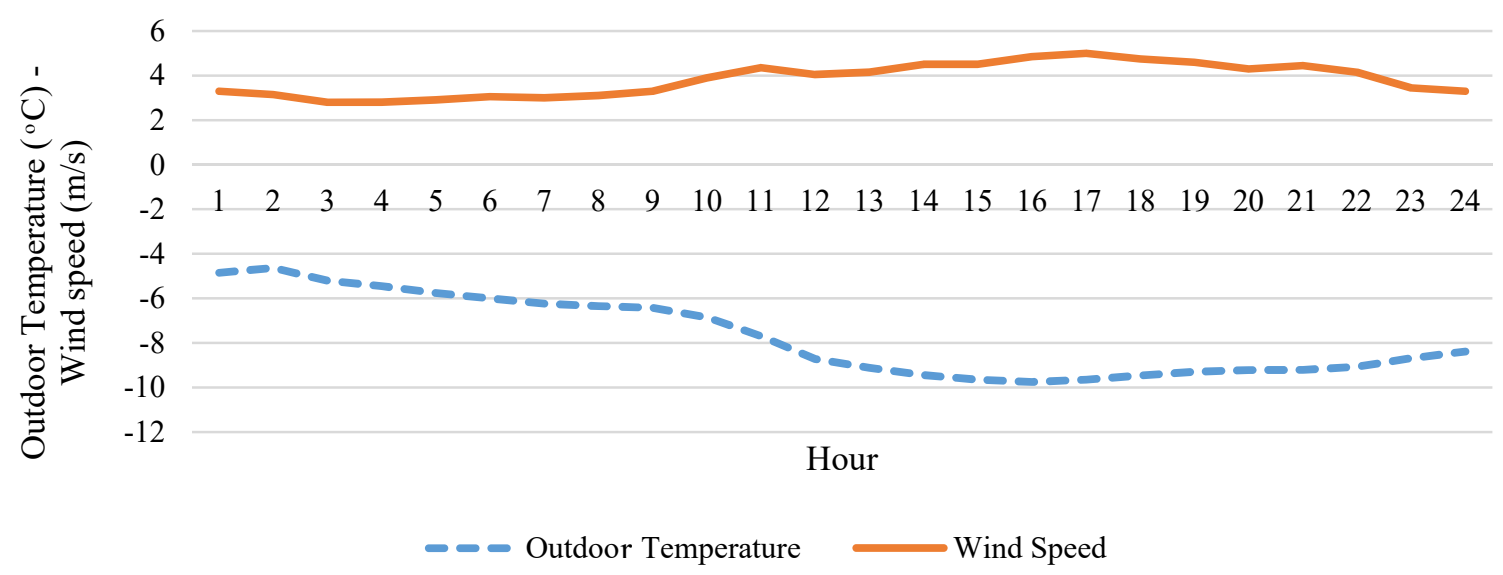

Figure 5-31: Outdoor temperature and wind speed during the sample day 


\subsubsection{Conventional On-Off Controller}

Figure 5-32 illustrates the house thermal demand $(\mathrm{kWh})$ under the effects of solar irradiations, outdoor temperature and wind speed as the outdoor disturbances as well as appliances/lighting and occupant as the internal gains/disturbances when $22^{\circ} \mathrm{C}$ is selected as the indoor set point.

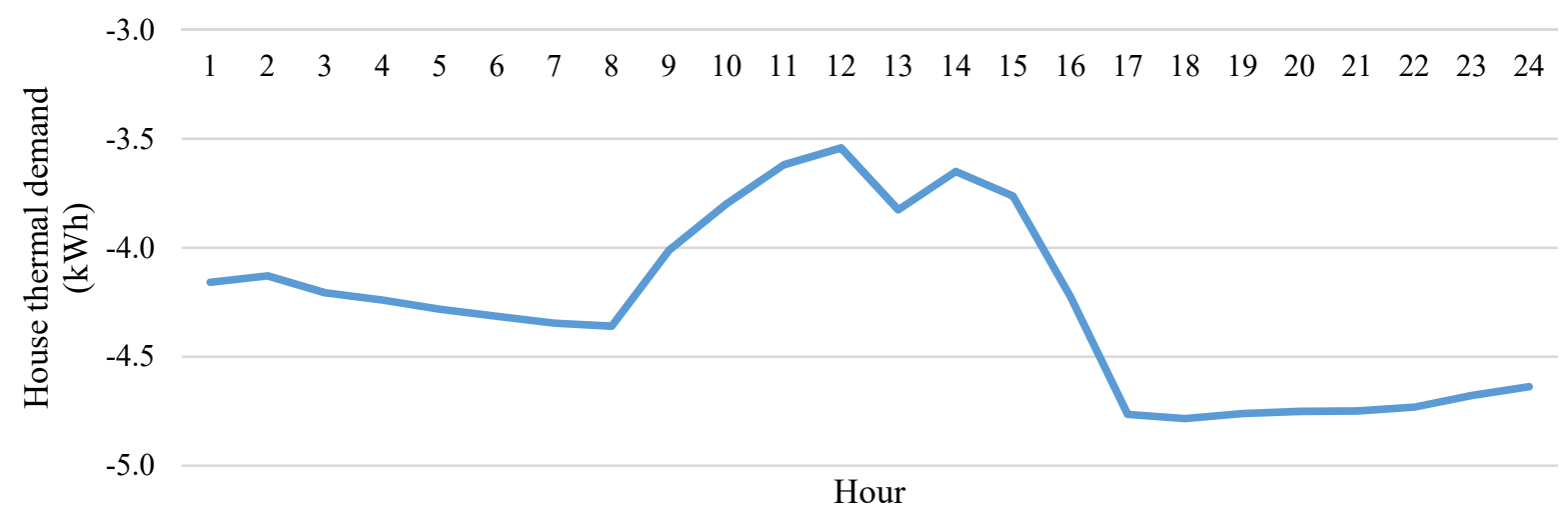

Figure 5-32: Case study house thermal demand during the sample day

Figure 5-33 shows ASHP energy demand when $22^{\circ} \mathrm{C}$ and 5 mins are selected as the constant indoor set point temperature and constant HVAC system operating time step, respectively, for entire day. As it is evidenced by Figure 5-33, the ASHP demand increases with increasing house thermal demand at the beginning and end of the day (when the outdoor air temperature is cold and there is no solar radiation); however, it decreased in the middle of the day when house thermal demand was reduced because of the thermal effects of solar radiations on different wall and windows surfaces and internal solar gains.

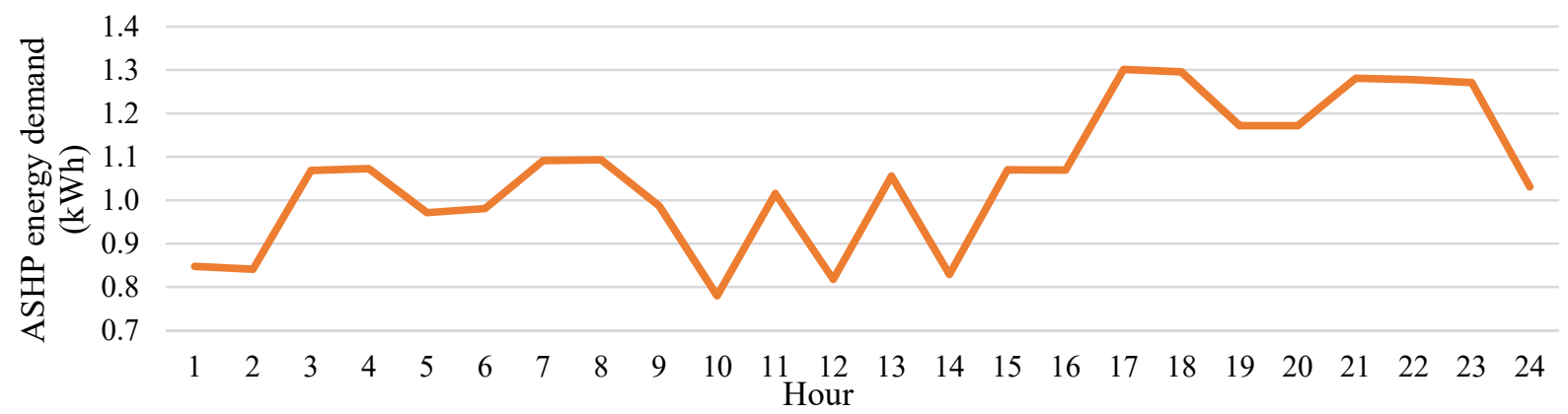

Figure 5-33: ASHP energy demand during the sample day 
The total ASHP energy demand and the associated cost were $25.42(\mathrm{kWh})$ and $\$ 4.34$ for the sample day using conventional on-off controller.

\subsubsection{Demand MPC Controller}

The Demand MPC controller is set up based on the information presented in Table 5-6.

Table 5-6: Demand MPC controller set up information

\begin{tabular}{|c|c|c|c|c|c|c|c|}
\hline Min & Max & $\begin{array}{c}\text { Min } \\
\text { allowable }\end{array}$ & $\begin{array}{c}\text { Max } \\
\text { allowable }\end{array}$ & Min & Max & Min & Max \\
\hline set & set & zone & zone & time & time & time & time \\
\hline point & point & temperature & temperature & step & step & horizon & horizon \\
\hline$\left({ }^{\circ} \mathrm{C}\right)$ & $\left({ }^{\circ} \mathrm{C}\right)$ & $\left({ }^{\circ} \mathrm{C}\right)$ & $\left({ }^{\circ} \mathrm{C}\right)$ & (min) & $(\min )$ & (hr) & (hr) \\
\hline 20 & 24 & 18 & 26 & 1 & 30 & 3 & 24 \\
\hline
\end{tabular}

Figure 5-34 shows HVAC system minimum energy cost and optimum operating time step when control time horizon increases from 3 hours to 24 hours. Figure 5-35 demonstrates the average energy cost of HVAC system. Based on this figure, $\mathrm{TH}=15 \mathrm{mins}$ is selected as the optimum control time horizon at first iteration of MPC controller.

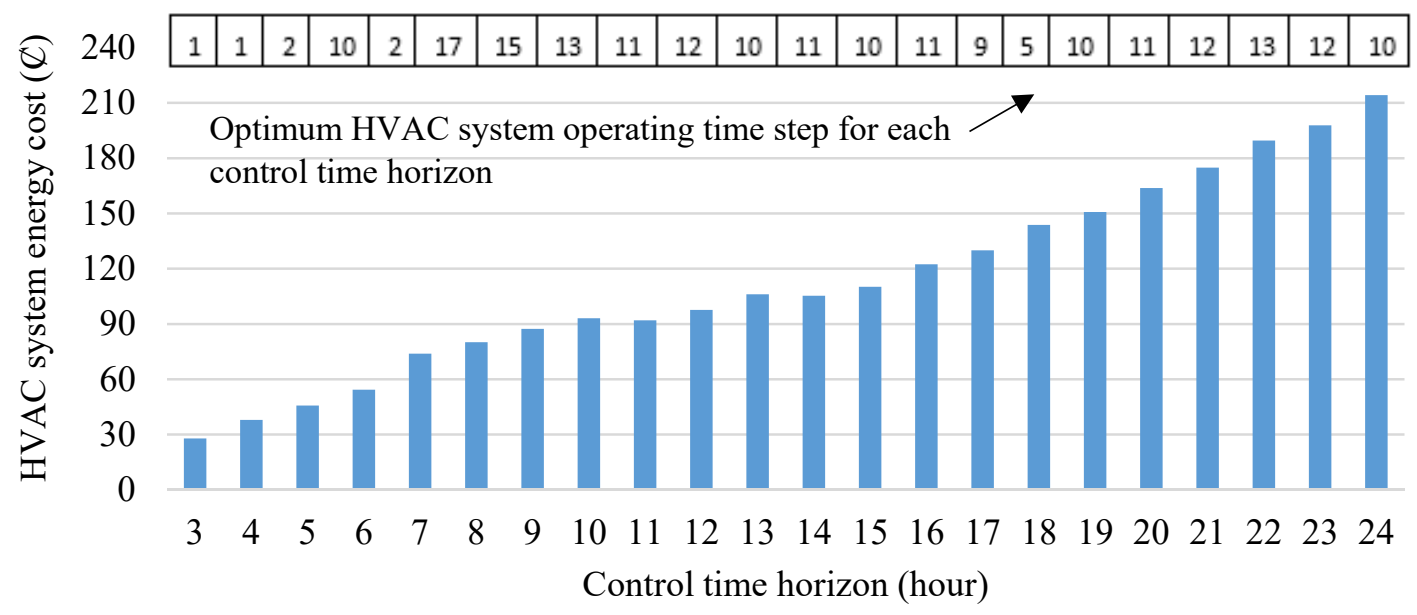

Figure 5-34: HVAC system minimum energy cost and optimum operating time step 


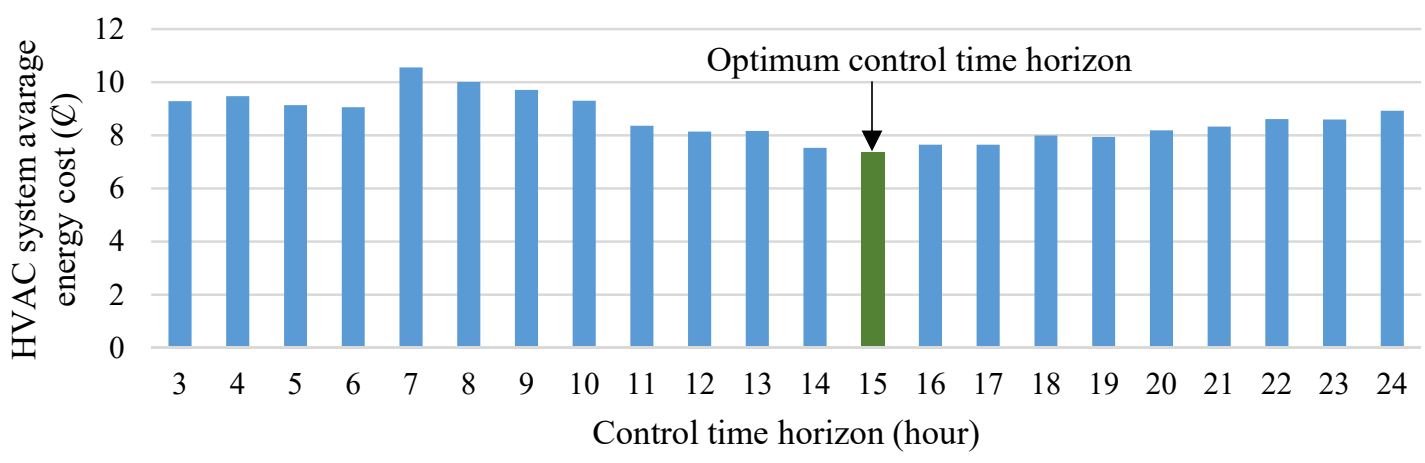

Figure 5-35: Average of HVAC system energy cost in each control TH

The optimum set point and corresponding HVAC system energy demand patterns/profiles, in which the HVAC system energy cost is minimum, along with TOU energy price scheme are demonstrated in Figure 5-36 when Demand MPC controller is employed for covering the entire sample day. As it is evidenced by Figure 5-36, higher indoor set points have been chosen by Demand MPC controller during off-peak hours to pre-heat the house while lower indoor set points have been selected during peak hours to decrease the energy cost. Since outdoor temperature gets colder from $19 \mathrm{pm}$ to $24 \mathrm{pm}$ in the sample day (which results in increasing the house thermal demand), lower indoor set points have been selected during these hours to decrease the HVAC system energy demand and energy cost while maintaining thermal comfort. Considering the fact that the house thermal demand is reduced at the middle of the day (because of internal and external effects and gains of solar radiation), higher indoor set points have been selected by MPC controller during these hours to maximize thermal energy storage inside the house and exploitation of less expensive renewable energy sources. 


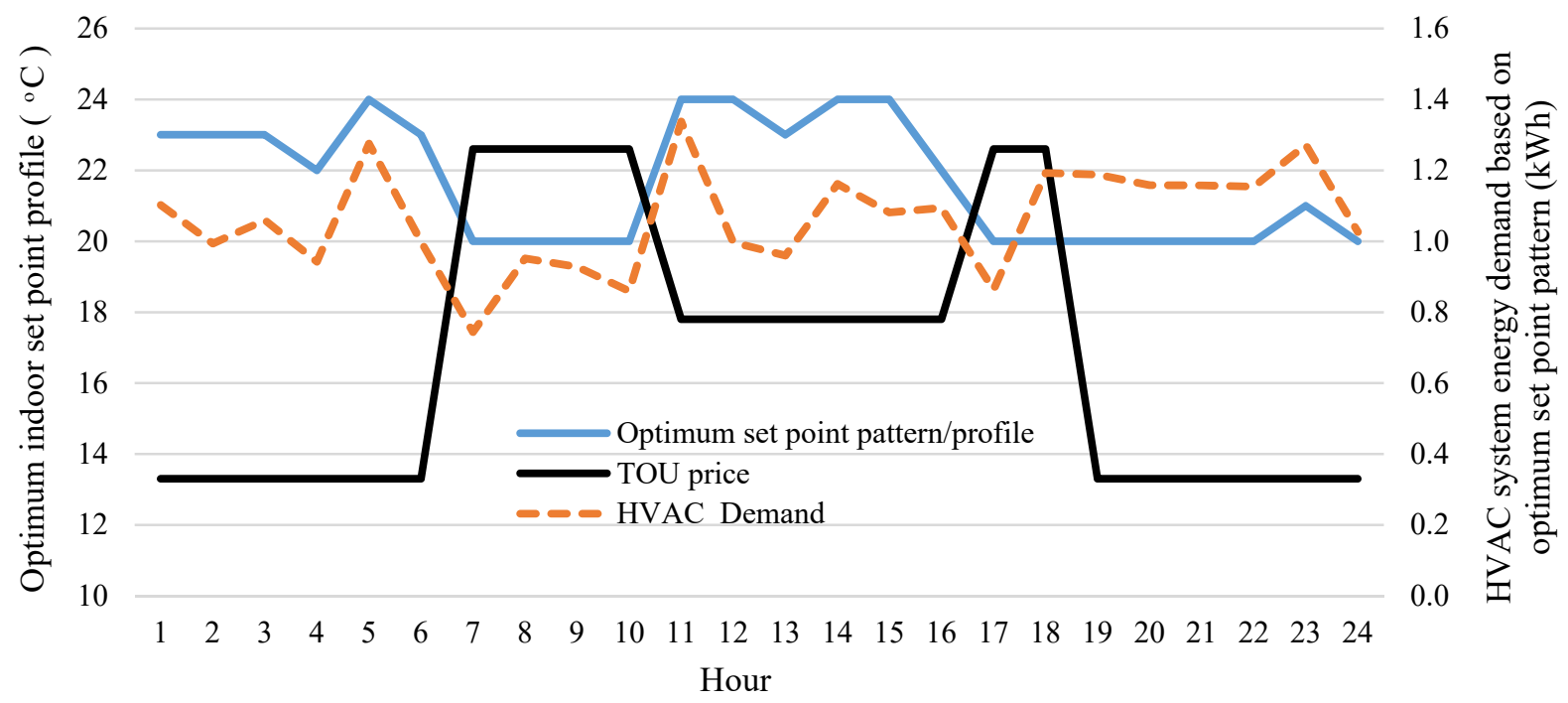

Figure 5-36: Optimum indoor set point pattern along with corresponding HVAC system energy demand and TOU energy price scheme

The behavior of HVAC system energy demand is complex because it is influenced by the combined effects of house thermal demand and optimum indoor set point at each hour. In the developed control model, higher indoor set points are selected by MPC controller for the hours in which house thermal demand is low and vice versa. Therefore, the behavior of HVAC system energy demand is almost moderate except for the hours in which maximum user-defined set point is selected either for pre-heating the house or maximizing the exploitation of renewable energies. The next step after calculating the optimum HVAC system energy demand is to calculate the optimum powers drawn from different energy sources. Figure 5-37 illustrates the renewable energy generated by PV and WT using the outdoor weather information of the sample day depicted in Figures 5-30 and 5-31. 


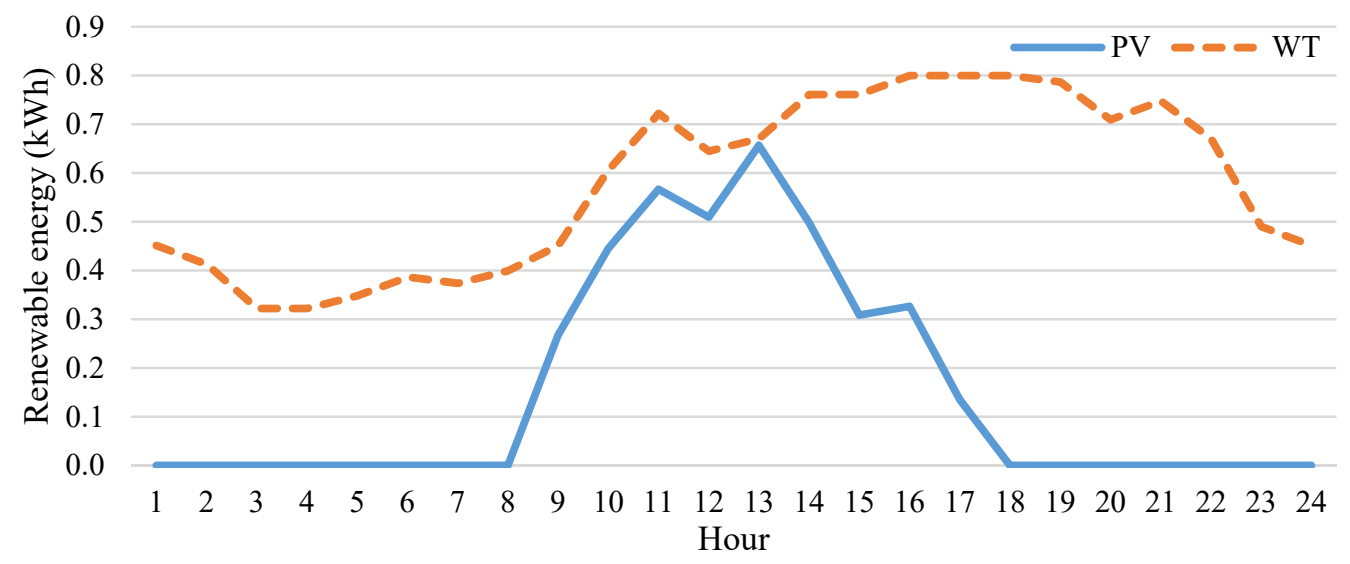

Figure 5-37: Renewable energy generated by PV and WT during the sample day

Figure 5-38 shows the optimum powers drawn from different energy sources for supplying the HVAC system energy demand during the sample day.

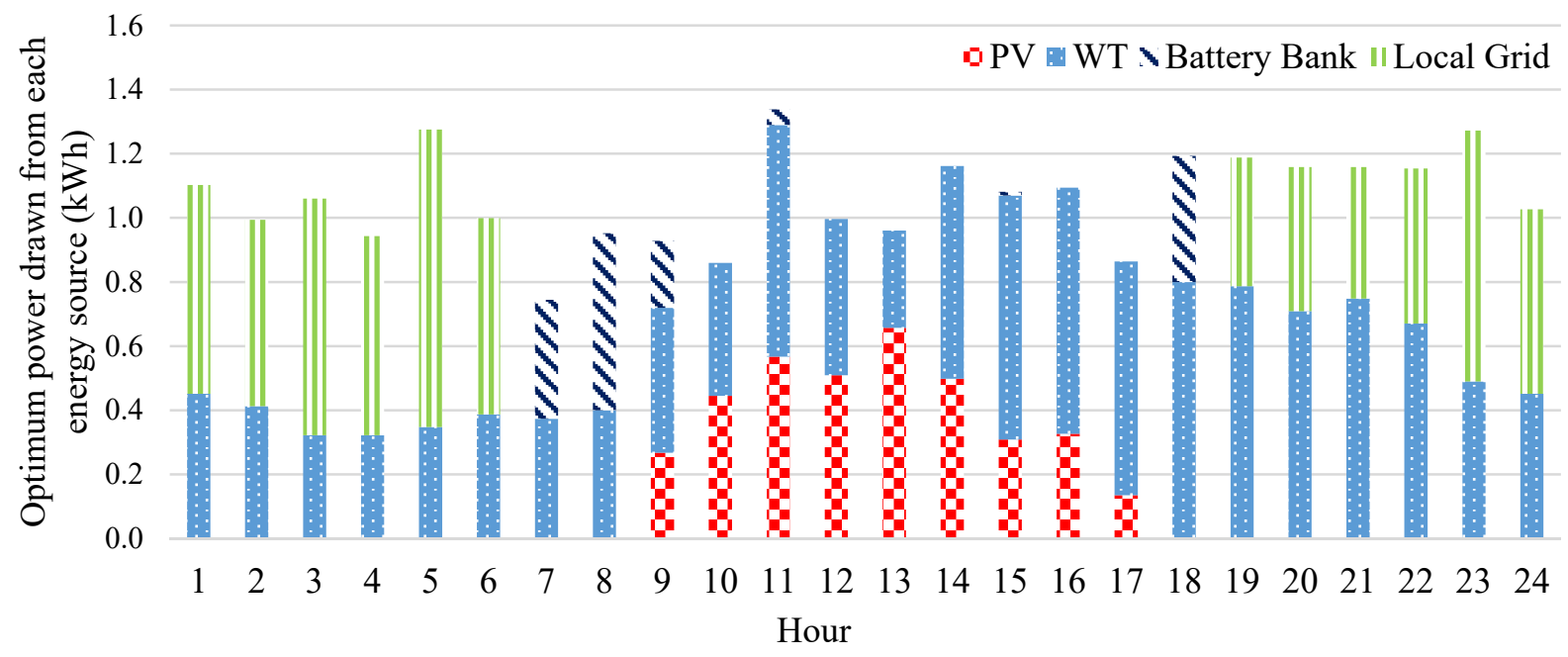

Figure 5-38: Optimum powers drawn from different energy sources during the sample day

Based on the weighting factors of different energy sources, which described in Table 5-5, PV and WT energy sources were used as the first priority for supplying the HVAC system energy demand. However, battery bank was only used during mid- and peak hours when renewable energy sources were not able to cover the HVAC system demand. The local grid was only utilized during off-peak hours (when electricity price is low) when there was lack of renewable energy for supplying the 
HVAC system demand. In this simulation, it is assumed that the house energy system is not supported by solar community.

Figure 5-39 presents the contribution of each energy source in supplying the HVAC system energy demand.

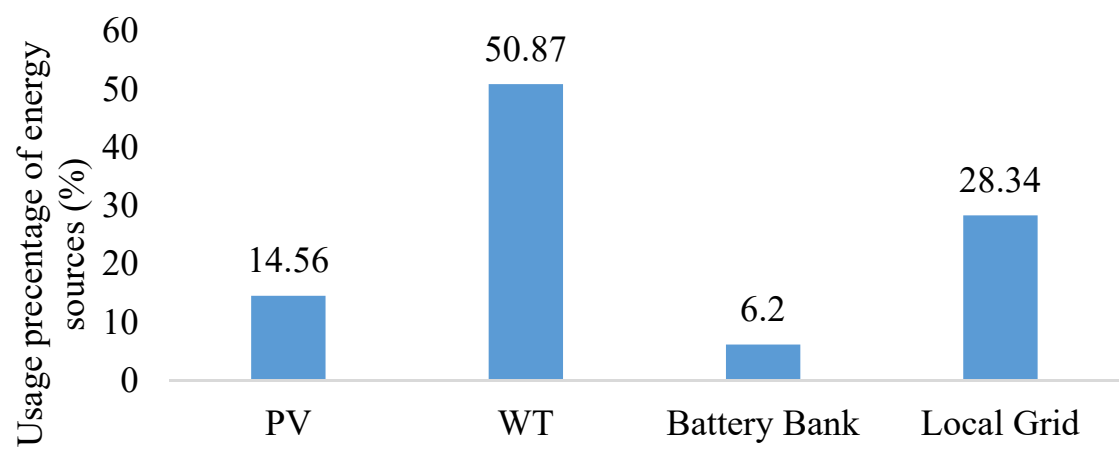

Energy sources

Figure 5-39: Contribution of each energy source in supplying the HVAC system energy demand

Because of the consistent and relatively strong wind in the sample day, this energy source had the most contribution in supplying the HVAC system energy demand.

After employing the Demand MPC controller, the HVAC system energy costs when utilizing combined renewable and battery bank systems, only battery bank system, and without using renewable and battery bank systems (only using local grid) were $\$ 1.89, \$ 3.37$ and $\$ 3.69$, respectively. Figure 5-40 shows the obtained savings (versus conventional on-off controller) on the HVAC system energy cost after implementing the Demand MPC controller on the sample day. Based on this figure, running the MPC controller by taking advantage of outdoor disturbances and preheating processes (which were described in detail in AISPG and CSPMG functions), offered $14.84 \%$ saving on the HVAC system energy cost while local grid used as the only energy source. 


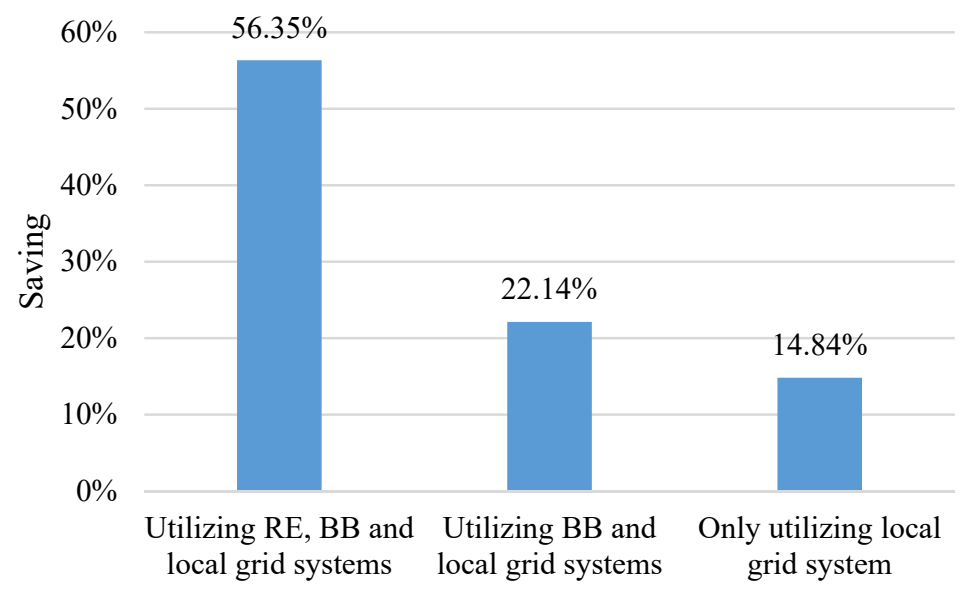

Figure 5-40: Savings on the HVAC system energy cost after implementing the Demand MPC controller

\subsubsection{Simulation Result for the Heating Season}

This section contains the simulation results for the whole winter season. The heating season simulation was performed using the weather information depicted in Figures 5-1 and 5-2. Weather information were read from metropolitan Toronto weather provided by the TRNSYS library. In this section, the effectiveness of Demand MPC controller on heating season is compared with three base case scenarios when PV, WT, battery bank and local grid are used as the energy sources. However, to highlight the productivity of Demand MPC controller, a simple rule-based (SRB) controller as an efficient HVAC controller is also used for comparing the energy cost saving results. SRB controller itself offers saving on the HVAC system energy cost since this controller adjusts indoor set point based on TOU pricing scheme. In order to check the performance of MPC under a hard condition, Demand MPC controller was employed without using PV, WT and battery bank as the energy sources (local grid was selected as the only energy source) when the energy cost saving results were compared with SRB controller.

\subsubsection{Base Case and Simple Rule-based Scenarios}

As described in Section 5.3, three different base case scenarios were considered for running the controller using conventional on-off controllers. In the first base case scenario, $20^{\circ} \mathrm{C}$ was selected 
as the indoor set point for the entire day while $22^{\circ} \mathrm{C}$ and $24^{\circ} \mathrm{C}$ were selected as the constant indoor set points in the second and third base case scenarios, respectively. A simple rule-based (SRB) controller (as an efficient HVAC controller) was also used to investigate the effectiveness of Demand MPC controller. In these simulations, local grid was used as the only energy source for suppling the HVAC system demand. Figure 5-41 demonstrates the indoor set point profiles based on three different base case scenarios and SRB controller.

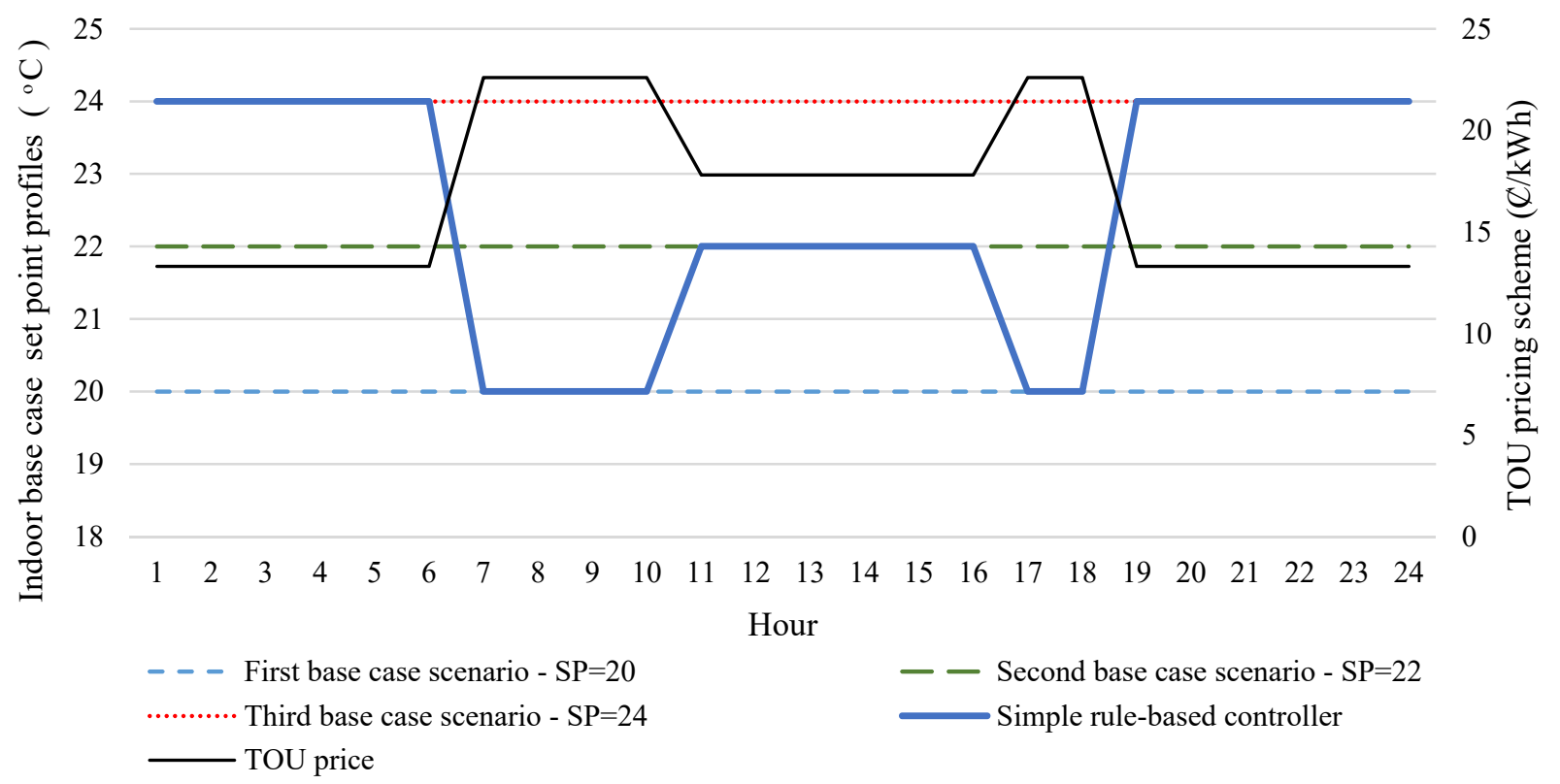

Figure 5-41: Indoor set point profiles base case different four scenarios

Figure 5-42 shows the energy cost of HVAC system by using three different base case scenarios (using conventional on-off controllers) and SRB controller in the heating season when 5 mins is selected as the HVAC system operating time step. Based on the simulation results, the total energy costs of HVAC system using the first, second, third scenarios and SRB controller for the heating season were $\$ 564.98, \$ 630.76, \$ 695.10$, and $\$ 610.01$, respectively. 


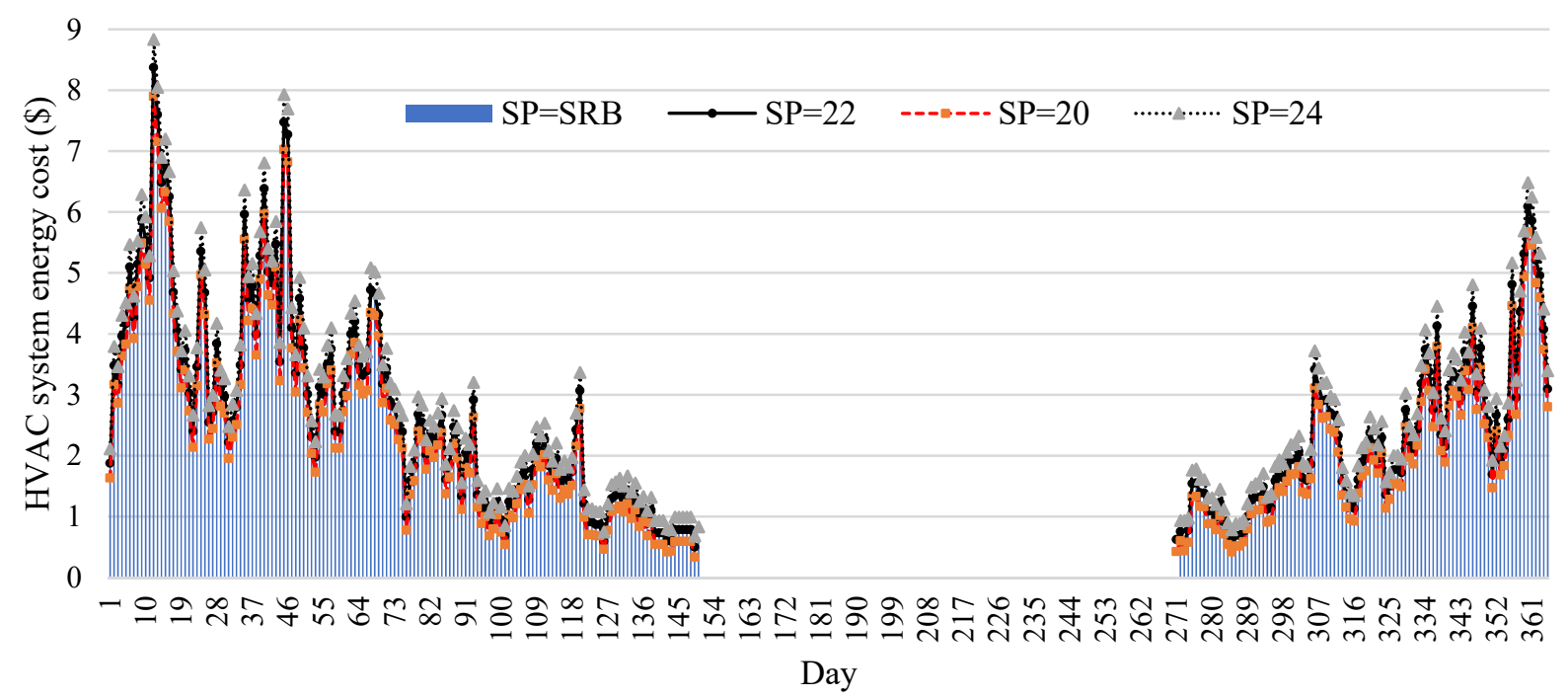

Figure 5-42: Energy cost of HVAC system by using different base case scenarios in heating season

\subsubsection{Employing Demand MPC Control Using PV, WT, and Battery Bank Systems}

After running the base case and SRB scenarios in the heating season, Demand MPC controller is employed to optimize the performance of HVAC system for the heating season. The total HVAC system energy cost after employing MPC controller is found \$ 325.27. In Figure 5-43, the total HVAC system energy cost is compared with the total HVAC system energy cost in base case and SRB scenarios.

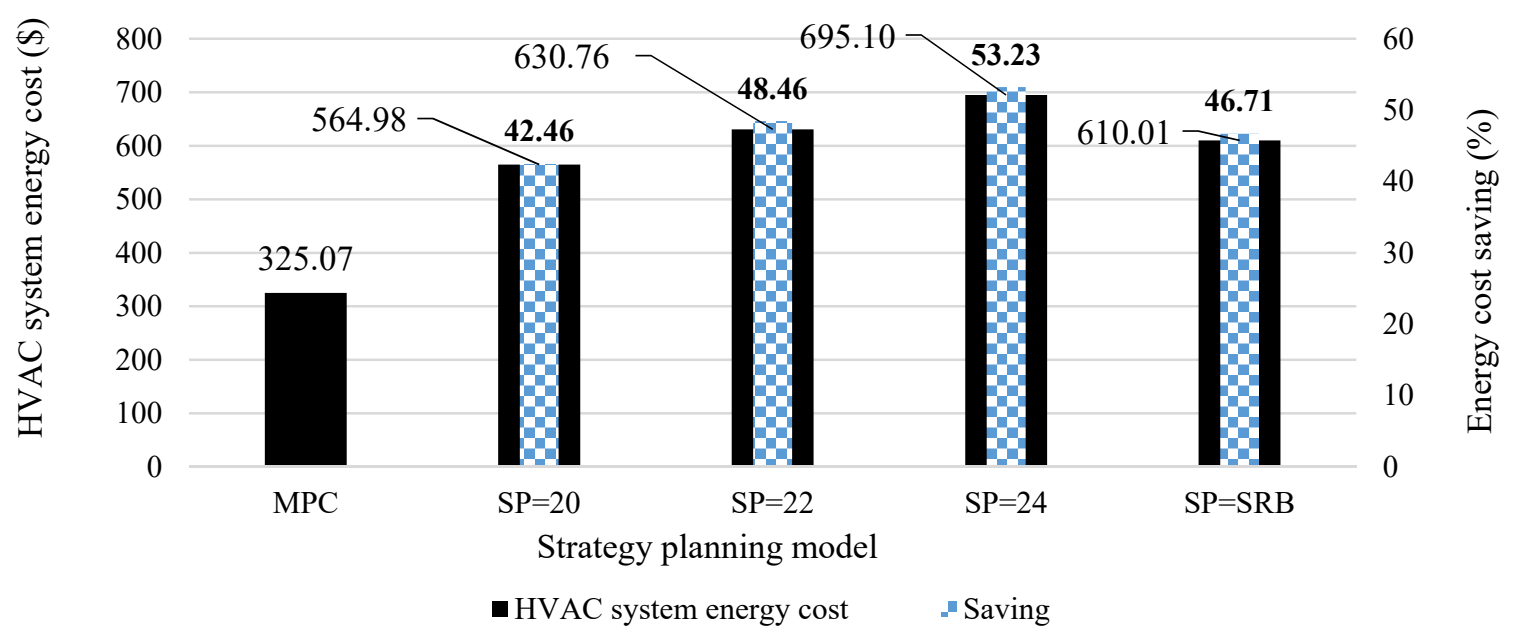

Figure 5-43: Comparing MPC result with different base case and SRB scenarios for the heating season 
The simple rule-based controller/scenario $(\mathrm{SP}=\mathrm{SRB})$ is selected as an efficient controller for detailed analysis of performance of MPC controller. In Figure 5-44, HVAC system energy cost in different days of heating season are compared between SRB scenario and MPC controller.

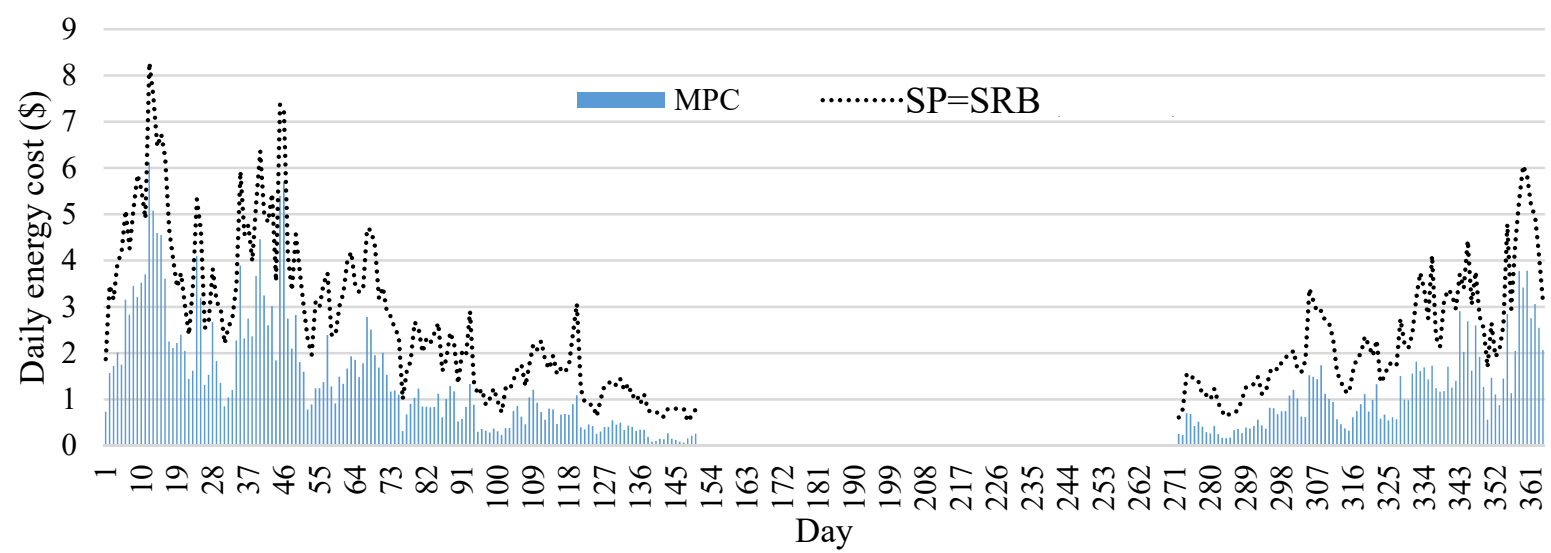

Figure 5-44: Comparing HVAC system energy cost based on SRB scenario and MPC controller during the heating season

Figure 5-45 illustrates the daily energy cost saving that calculated based on MPC and SRB energy cost curves (depicted in Figure 5-44) in different days of the heating season.

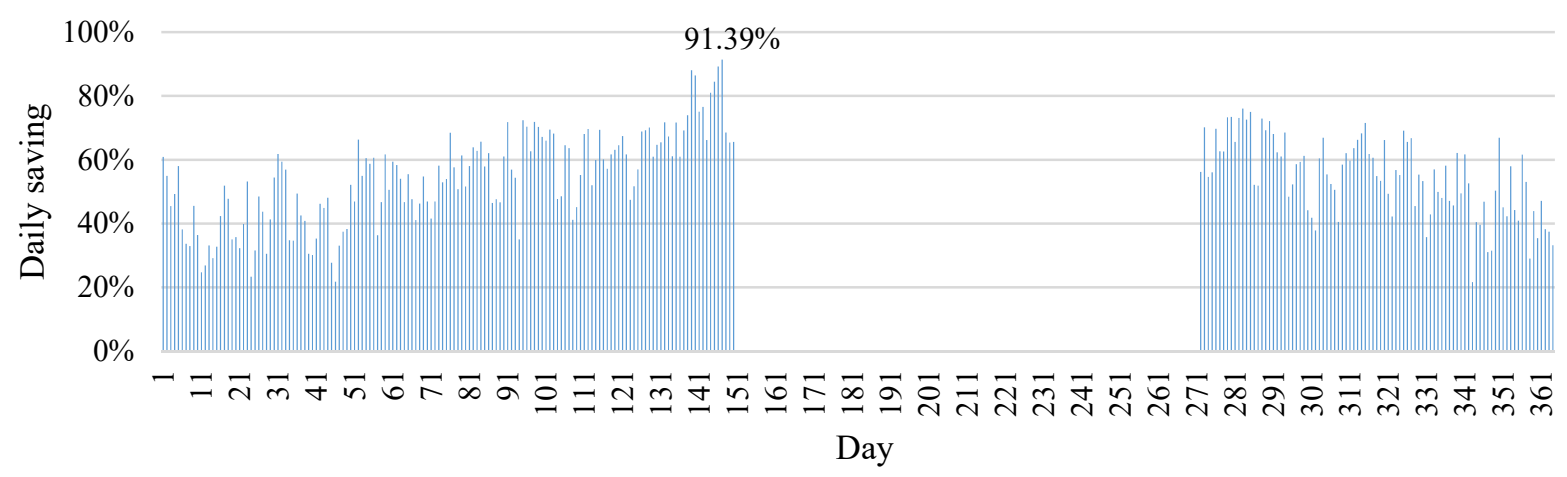

Figure 5-45: MPC additional saving on the HVAC system energy cost (comparing SRB scenario) during different days of heating season

Based on Figure 5-45, the maximum daily energy cost saving (91.39\%) happened on day 147 when the renewable sources had maximum participation in supplying the HVAC system demand. However, the minimum saving $(21.02 \%)$ happened on day 46 when the HVAC system demand/energy cost was too high and there was no effective participation of renewable sources in supplying the HVAC system demand due to the very cold weather condition (and lack of solar 
radiation) during this particular day. As it is evidenced by Figure 5-45, the rate of saving on HVAC energy cost by using MPC controller is generally increased as we move toward warmer days of the year because of higher participation of renewable sources in covering the HVAC demand. As depicted in Figure 5-43, the average 46.71\% saving (comparing SRB scenario) happened during the heating season by employing the MPC controller.

Figure 5-46 shows the HVAC system energy demand in the SRB scenario and after employing MPC controller during the heating season.

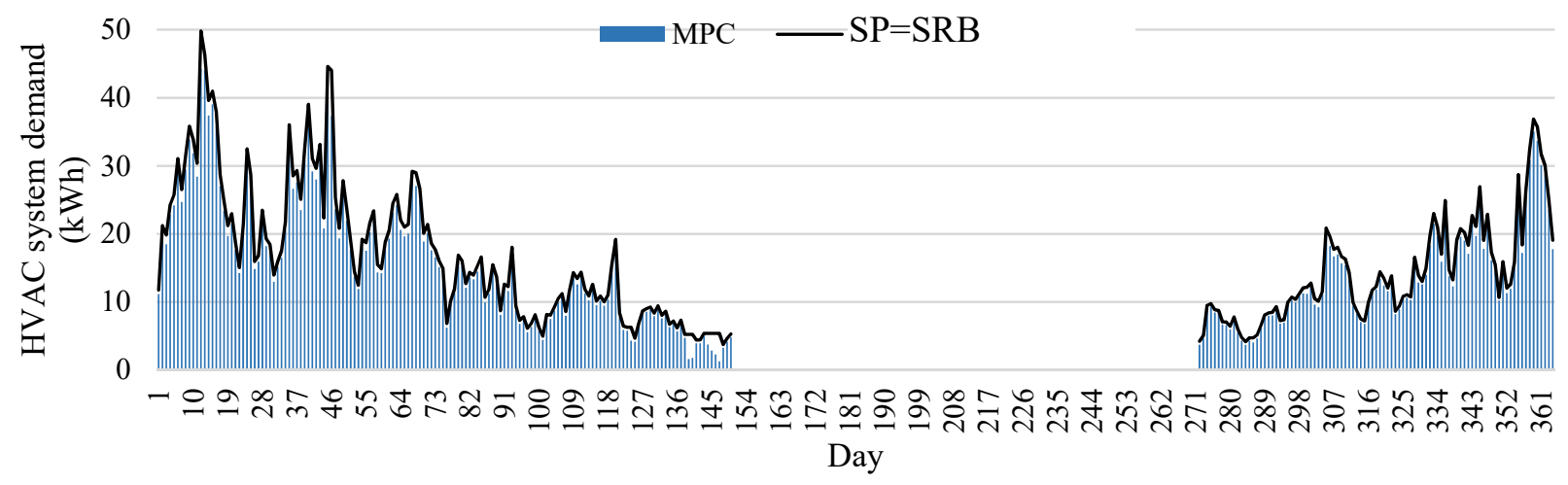

Figure 5-46: HVAC system energy demand based on SRB scenario and after employing MPC controller during the heating season

As it is evidenced from Figure 5-46, using the optimum HVAC system operating time step (calculated by MPC controller taking advantage of exhaustive optimization) reduced the HVAC system energy demand. Figure 5-47 illustrates the saving in the HVAC system energy demand calculated considering Figure 5-46 curves. Based on Figure 5-47, 75.84\% is the maximum saving in the HVAC system energy demand after employing MPC controller while $1.89 \%$ and $7.46 \%$ are the minimum and average savings, respectively, during the heating season.

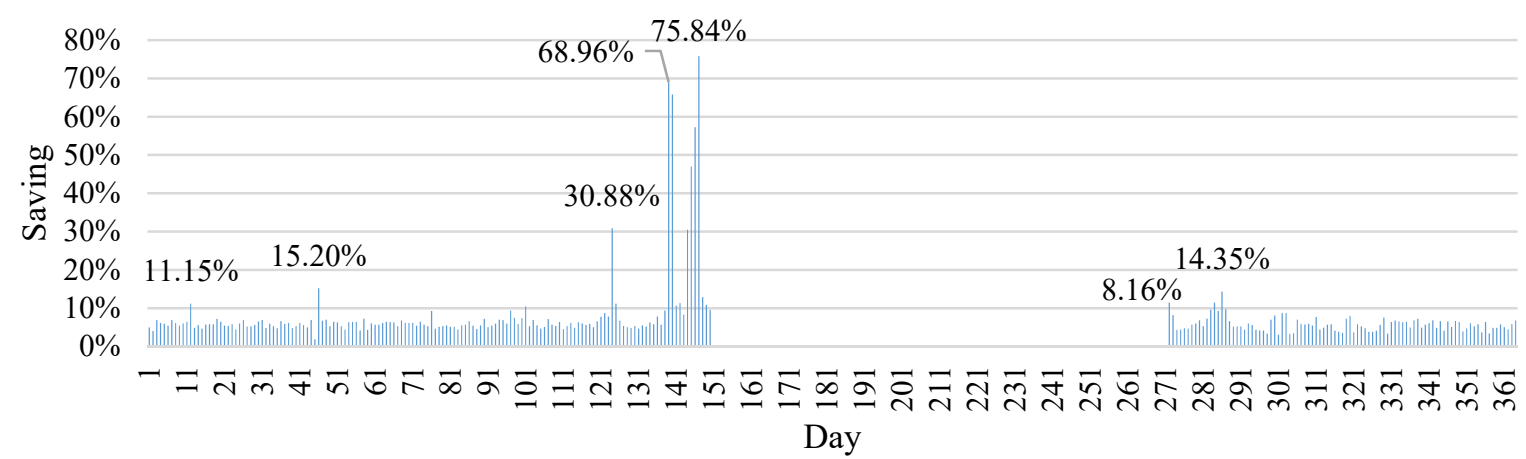

Figure 5-47: Saving on the HVAC system energy demand (comparing SRB scenario) after employing MPC controller during the heating season 
As shown in Figure 5-47, when the energy demand of HVAC system is too high (i.e., days 12 and 46) or too low (i.e., days 143 to 149), operating the HVAC system with optimum time step resulted in more reduction in HVAC system demand.

Figure 5-48 shows the optimum HVAC system operating time step that resulted in minimum HVAC system energy demand as well as the optimum HVAC system TS that resulted in minimum HVAC system energy cost in each day of the heating season when 24-hour is selected as the control time horizon.

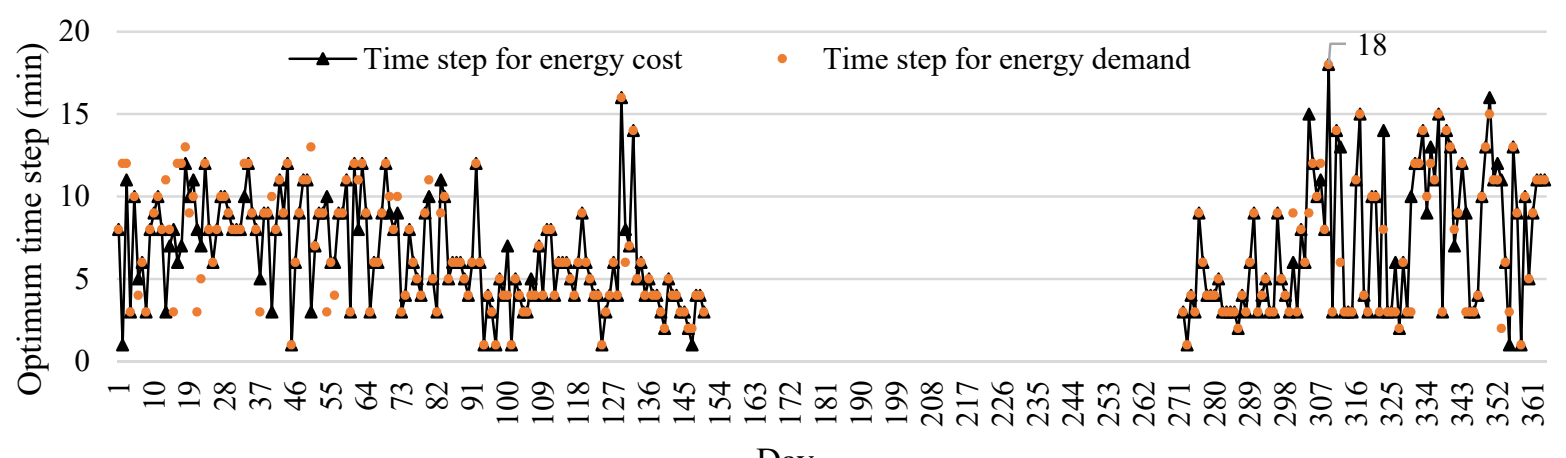

Day

Figure 5-48: Optimum HVAC system operating time steps during the heating season

Based on Figure 5-48, 1 min and 18 mins are selected as the minimum and maximum, respectively, of the optimum HVAC system operating time steps during the heating season. In other words, based on the house thermal model, HVAC system characteristics, and weather condition during the heating season, the time step higher than 18 mins mainly resulted in zone temperature violation and could not offer the optimum results.

\subsubsection{Employing Demand MPC Control without Using PV, WT, and Battery Bank Systems}

By removing PV, WT, and battery bank (as less expensive energy sources) from the house energy system, local grid/utility is the only source is used for covering the HVAC system demand. As a result, comparing the energy cost saving result of MPC controller with SRB scenario (SRB scenario sets indoor set points based on TOU pricing scheme to minimize the HVAC system energy cost) in the heating season, clearly, demonstrates the effectiveness of MPC controller in 
saving the energy cost. Figure 5-49 shows the total HVAC system energy cost after employing MPC and SRB controllers in the heating season.

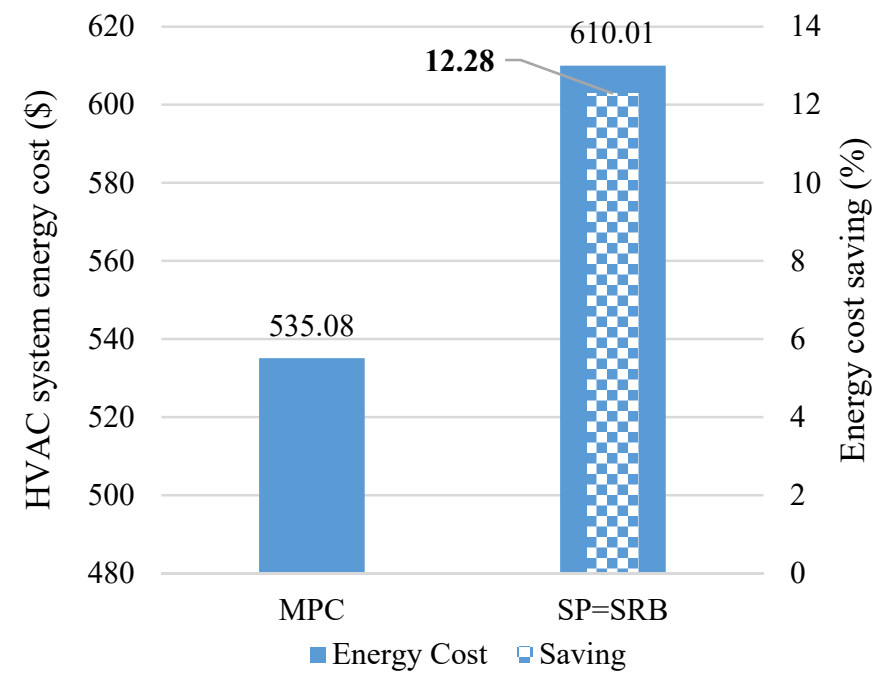

Figure 5-49: Comparing MPC result with SRB scenario in the heating season

Based on Figure 5-49, employing MPC controller resulted in 12.28\% additional energy cost saving in comparison with SRB scenario. HVAC system energy cost based on MPC controller and SRB scenario is depicted in Figure 5-50 for the different days of the heating season.

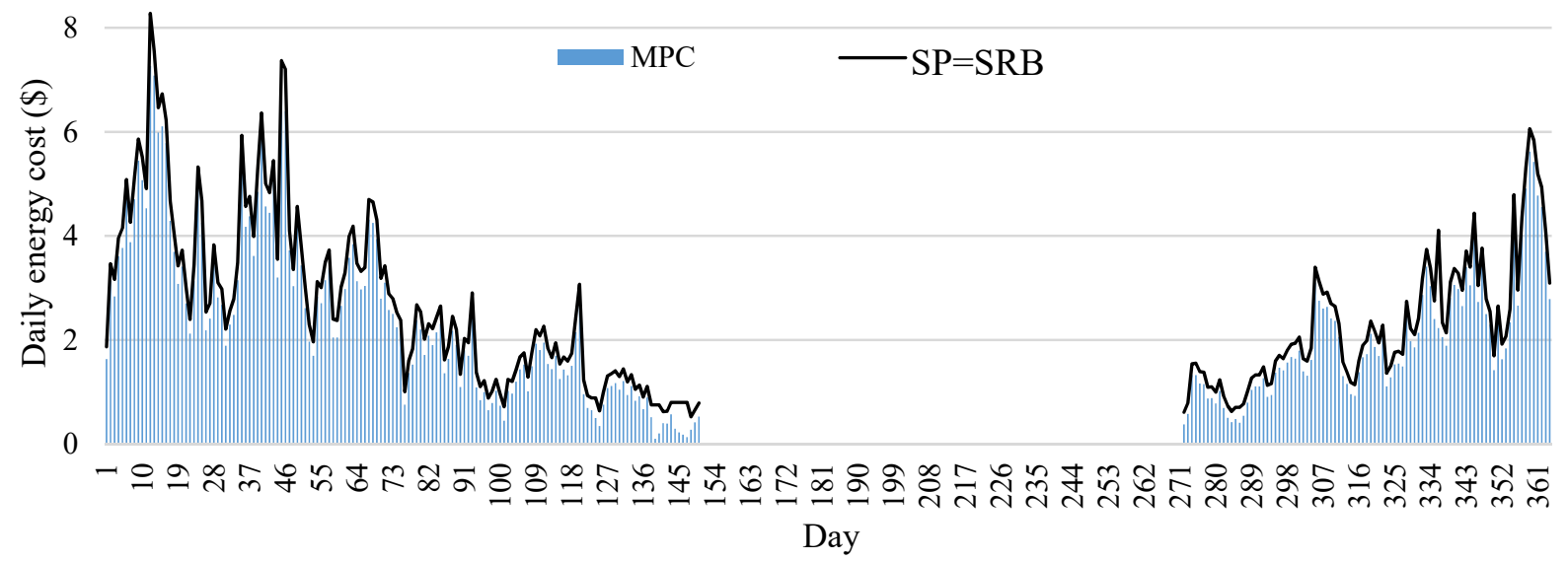

Figure 5-50: Comparing HVAC system energy cost based on MPC controller and SRB scenario in the heating season

Figure 5-51 illustrates the additional daily energy cost saving of MPC controller in comparison with SRB scenario during the different days of the heating season. 


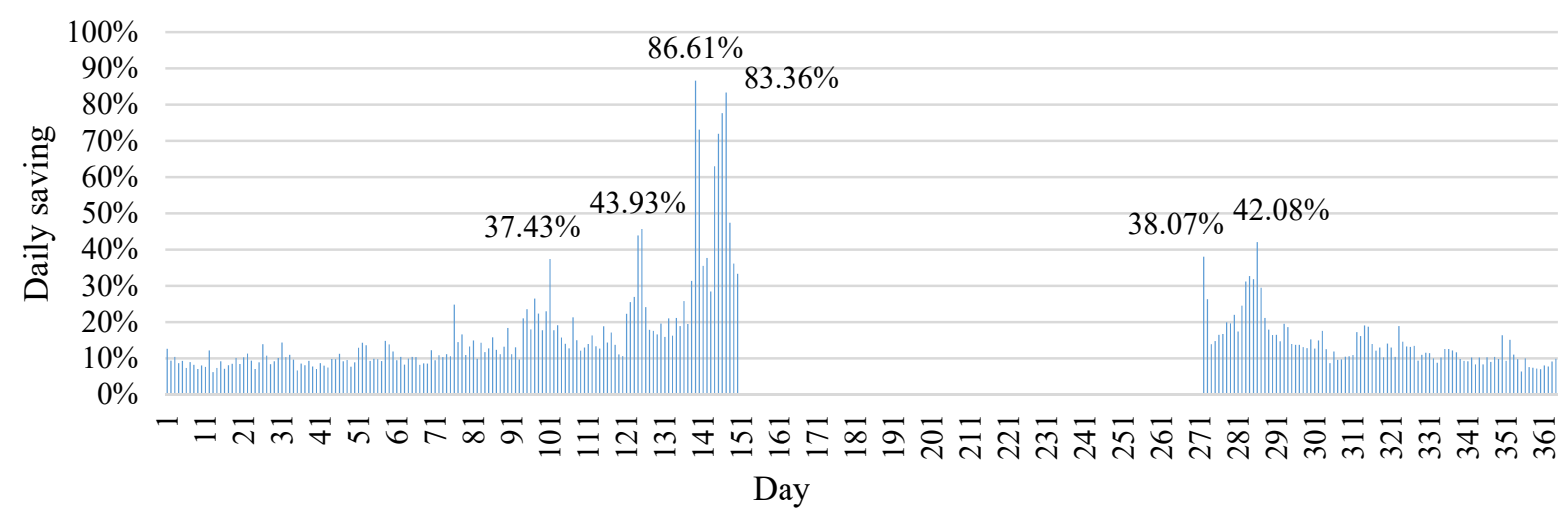

Figure 5-51: Additional daily energy cost saving of MPC controller in comparison with SRB scenario during the different days of the heating season

Based on Figure 5-51, the maximum saving (86.61\%) happened on day 141. This maximum saving obtained because of optimum indoor set point profile that was selected by MPC controller by considering the effects of outdoor disturbances during this particular day. Figure 5-52 shows outdoor temperature and wind speed while Figure 5-53 illustrates the solar radiation on the house different orientations in this particular day.

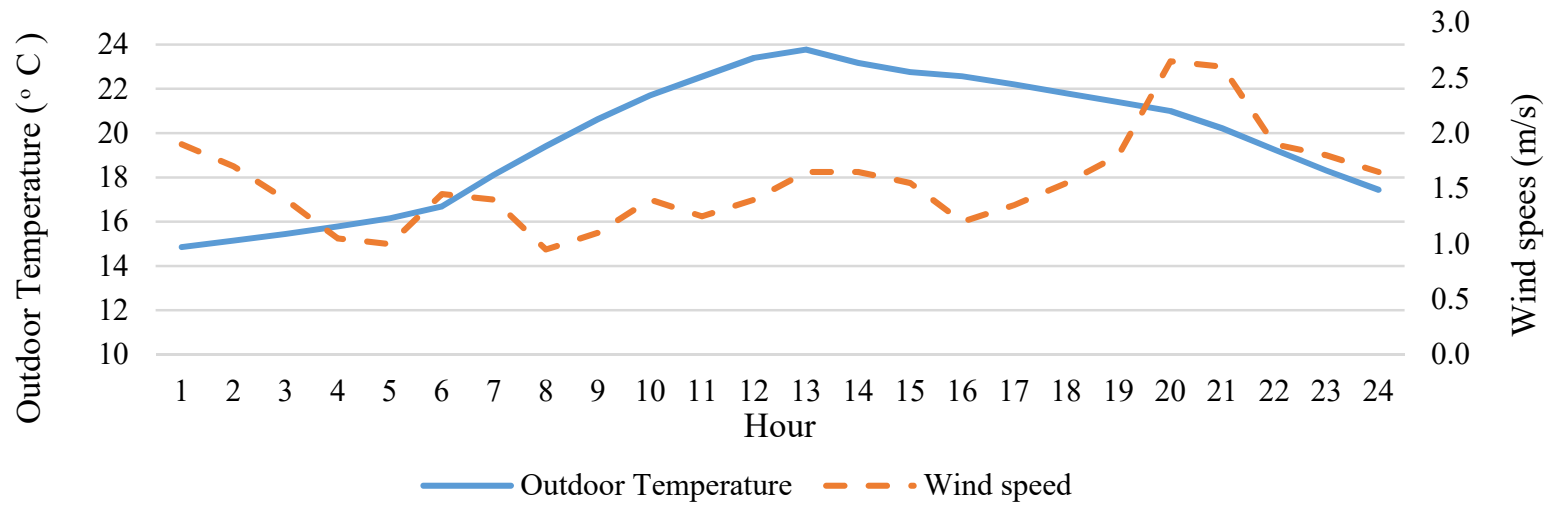

Figure 5-52: Outdoor temperature and wind speed in day 141 


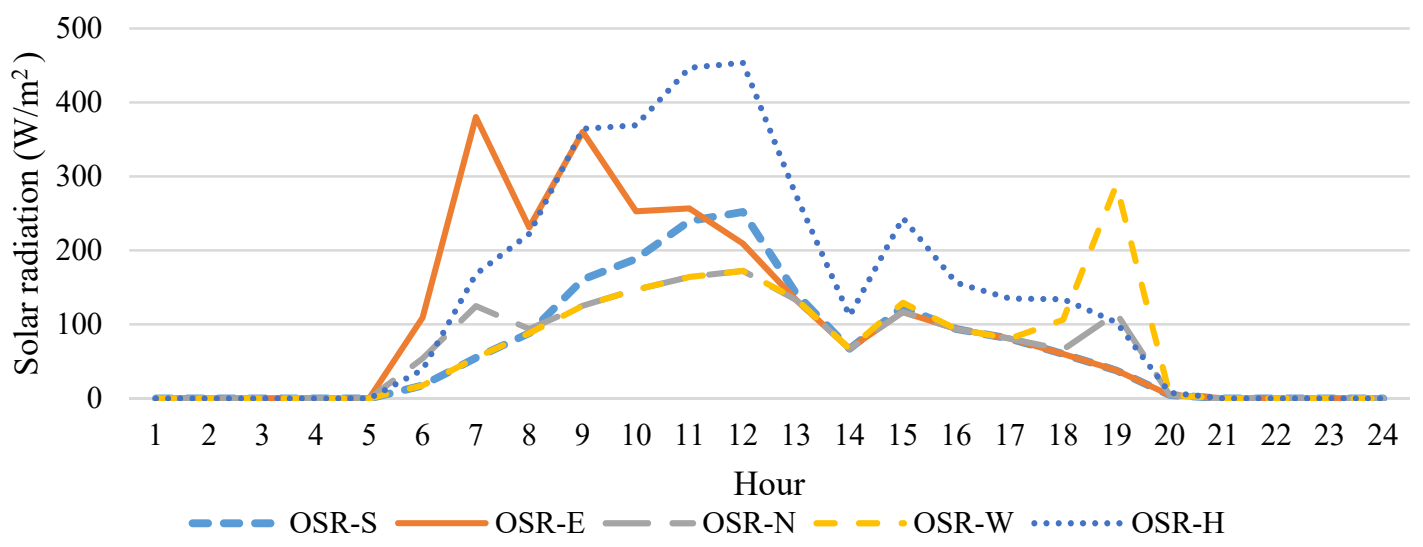

Figure 5-53: Solar radiation on the house different orientations in day141

Figure 5-54 illustrates the HVAC system energy demand obtained based on SRB scenario set point profile during this day. \$0.75 is calculated as the HVAC system energy cost using SRB scenario in this day.

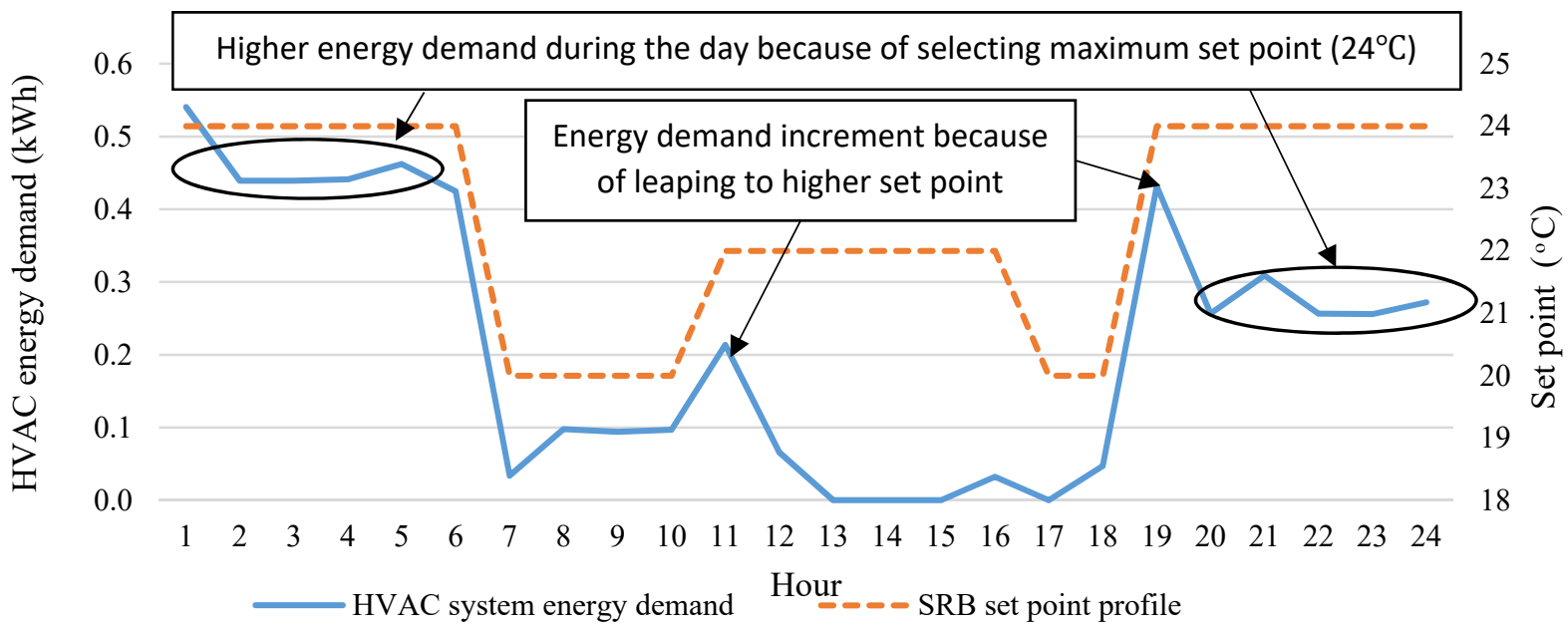

Figure 5-54: HVAC system energy demand based on SRB scenario

Figure 5-55 shows the optimum indoor set point profile and the corresponding HVAC energy demand after employing the MPC controller. Because of low thermal demand of the house during this warm winter day (due to relatively high outdoor temperature and solar heating gains), using pre-heating could not offer any saving on the HVAC system energy cost. As a result, $20^{\circ} \mathrm{C}$, as the minimum ASHRAE Comfort Standard range, is selected by the MPC controller as the optimum indoor set point for the entire day. $\$ 0.10$ is calculated as the minimum HVAC system energy cost (equal to $86.61 \%$ saving in comparison with the SRB scenario) taking advantage of MPC controller 
on this day. This significant saving happens since during the colder hours of the day (1 am to 6 am), selecting the minimum indoor set point of $20^{\circ} \mathrm{C}$ by $\mathrm{MPC}$ controller (instead of $24^{\circ} \mathrm{C}$ that is selected by SRB scenario) resulted in significantly lower HVAC system energy demand during these hours.

Furthermore, since on this day solar heat gains began at $5 \mathrm{am}$ (solar radiation at the east orientation of house reached its maximum at $7 \mathrm{am}$ ) and due to the relatively high outdoor temperature (particularly in the middle of the day), selecting the lowest set point by MPC controller resulted in no thermal demand from the house. As a result, HVAC system was completely off after 7 am leading to additional energy cost saving in comparison with SRB scenario.

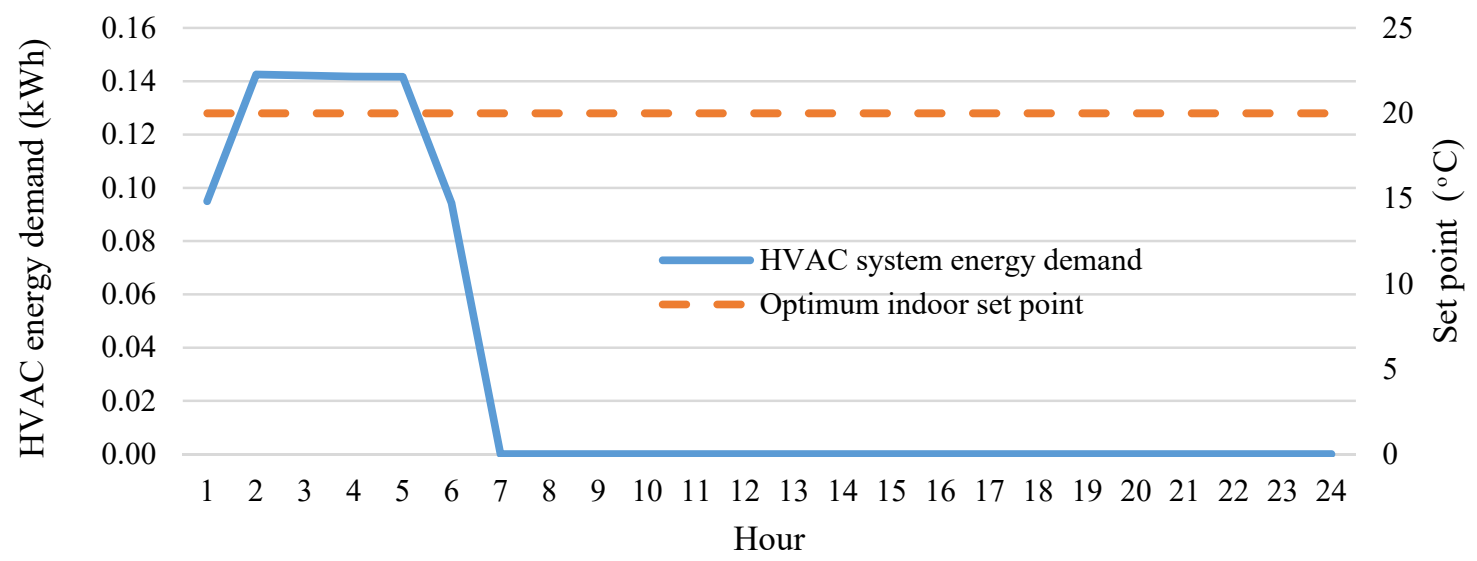

Figure 5-55: Optimum indoor set point profile and corresponding HVAC energy demand after employing the MPC controller

\subsection{Conclusion}

In this chapter, the development of the second MPC as a novel model-based predictive controller was discussed in detail. This novel MPC controller utilizes an intelligent algorithm (including two SDSSs) to reduce the MPC computational (optimization) time significantly. The developed MPC controller takes advantage of on-site energy generation and storage, dynamic outdoor disturbances and time of use (TOU) energy prices to identify the most useful indoor set point profiles (which reduced the HVAC system demand and energy cost).

Different optimization schemes (exhaustive search method and linear optimization) and smart decision support systems have been utilized in the MPC controller intelligent algorithm to find the optimum results (including time horizon, time step, and indoor set point profile) and reduce the 
optimization computational time. Based on the simulation results, employing the MPC controller on a case study house offered significant savings on HVAC system energy cost. In the heating season, based on the SRB controller/scenario (that was selected as an efficient controller), a maximum of $91.39 \%$ and an average of $46.71 \%$ energy cost saving on the HVAC system were achieved by employing the renewable power, battery bank, and MPC controller. After removing renewable power and battery bank from the case study house energy systems, utilizing the MPC controller resulted in a maximum of $86.61 \%$ and an average of $12.28 \%$ additional saving on the HVAC system energy cost (in comparison with SRB scenario) in the heating season. The summary of the MPC controller energy cost saving results is presented in Table 5-7.

These results verify the superior performance/effectiveness of the developed MPC controller in comparison with the base case (conventional on/off controllers) and SRB (as an efficient HVAC controller) scenarios.

Table 5-7: Summary of the MPC controller energy cost saving with and without using PV, WT and BB systems

\begin{tabular}{|c|c|c|c|c|c|}
\hline Scenarios & $\begin{array}{c}\text { First base } \\
\text { case scenario } \\
\mathrm{SP}=20^{\circ} \mathrm{C}\end{array}$ & $\begin{array}{c}\text { Second base } \\
\text { case scenario } \\
\mathrm{SP}=22^{\circ} \mathrm{C}\end{array}$ & $\begin{array}{c}\text { Third base } \\
\text { case scenario } \\
\mathrm{SP}=24^{\circ} \mathrm{C}\end{array}$ & $\begin{array}{c}\text { Simple rule- } \\
\text { based } \\
\text { scenario } \\
\mathrm{SP}=\mathrm{SRB}\end{array}$ & $\begin{array}{c}\text { Simple rule- } \\
\text { based } \\
\text { scenario } \\
\mathrm{SP}=\mathrm{SRB}\end{array}$ \\
\hline $\begin{array}{c}\text { Energy } \\
\text { Sources }\end{array}$ & $\begin{array}{c}\mathrm{PV}, \mathrm{WT}, \mathrm{BB} \\
\text { and local } \\
\text { grid }\end{array}$ & $\begin{array}{c}\mathrm{PV}, \mathrm{WT}, \mathrm{BB} \\
\text { and local } \\
\text { grid }\end{array}$ & $\begin{array}{c}\mathrm{PV}, \mathrm{WT}, \mathrm{BB} \\
\text { and local } \\
\text { grid }\end{array}$ & $\begin{array}{c}\mathrm{PV}, \mathrm{WT}, \mathrm{BB} \\
\text { and local } \\
\text { grid }\end{array}$ & $\begin{array}{c}\text { only local } \\
\text { grid }\end{array}$ \\
\hline $\begin{array}{c}\text { MPC } \\
\text { Energy Cost } \\
\text { Saving (\%) }\end{array}$ & 42.46 & 48.46 & 53.23 & 46.71 & 12.28 \\
\hline $\begin{array}{c}\text { MPC } \\
\text { Energy Cost } \\
\text { Saving (\$) }\end{array}$ & 239.71 & 305.49 & 369.83 & 284.74 & 74.93 \\
\hline
\end{tabular}




\subsection{References}

5.1. L. Prez-Lombard, J. Ortiz, and C. Pout, "A review on buildings energy consumption information," Energy and Buildings, 2008.40(3): p.394-398.

5.2. Huang, H., Chen, L., Hu, E., " A new model predictive control scheme for energy and cost savings in commercial buildings: An airport terminal building case study", Building and Environment, 89 (2015.89: p. 203-216

5.3. Afram, A., Janabi-Sharifi, Gray-box modeling and validation of residential HVAC system for control system design, Applied Energy, 2015.137: p. 134-150

5.4. Freire, Z., Oliveira, G., Mendes, N., "Predictive controllers for thermal comfort optimization and energy savings, Energy and Buildings, 2008.40:p. 1353-1356.

5.5. Afram, A., Janabi-Sharifi, F., F., Theory and application of HVAC control systems - A review of model predictive control (MPC), Building and Environment, 2014.72: p. 343-355.

5.6. Shengwei, W., X. Xinhua, and H., Gongsheng, Robust MPC for temperature control of airconditioning systems concerning on constraints and multitype uncertainties. Build Serv Eng Res Technol, 2010. 31(1): p. 39-55.

5.7. Ma, J., Qin, J., Salsbury, T., Xu, P., "Demand reduction in building energy systems based on economic model predictive control. Chemical Engineering Science, 2011, 67 (1): p. 92100.

5.8. Prívara, S., Siroky, J., Ferkl, L., Cigler, J., "Model predictive control of a building heating system: The first experience. Energy \& Buildings, 2011, 43 (2): p. 564-572.

5.9. Moroşan, P.D., Bourdais, B., Dumur, D., Buisson, J., "Building temperature regulation using a distributed model predictive control", Energy \& Buildings, 2010, 42 (9): p. 1445-1452

5.10. Huang, H., Chen, 1., Hu, E., "new model predictive control scheme for energy and cost savings in commercial buildings: An airport terminal building case study', Building and Environment, 2015.89: p. 203-216

5.11. Hilliarda, C., Swana, L., Kavgica, M., et al., "Development of a whole building model predictive control strategy for a LEED silver community college", Energy and Buildings, 2016.111: p. 224-232

5.12. Bruni, G., Cordiner, G, Mulone, V. Sinisi,V., "Energy management in a domestic microgrid by means of model predictive controllers", Energy 2016.108: p. 119-131.

5.13. Razmara, M., Maasoumy M., Shahbakhti, M., Robinett, RD., "Optimal exergy control of building HVAC system”, Applied Energy, 2015.156: p. 555-565.

5.14. Lee, Y., Horesha, R., Libertib, L., "Optimal HVAC control as demand response with onsite energy storage and generation system”, Energy Procedia, 2015.78: p. 2106 - 2111

5.15. Ascionea, C., Biancoa, N., Stasioa, C., et al., "Simulation-based model predictive control by the multi-objective optimization of building energy performance and thermal comfort", Energy and Buildings, 2016.111: p.131-144.

5.16. Mirakhorli, A., Dong, B., "Occupancy behavior based model predictive control for building indoor climate-A critical review", Energy and Buildings, 2016.129: p. 499-513. 
5.17. Schibuola, L., Scarpa, M., Tambani, C., "Demand response management by means of heat pumps controlled via real time pricing", Energy and Buildings, 2015.90: p. 15-28

5.18. West, S., Ward, J., Wall, J., "Trial results from a model predictive control and optimization system for commercial building HVAC", Energy and Buildings, 2014.72: p. 271-279.

5.19. Dobbs, J., Hencey B., "Model predictive HVAC control with online occupancy model", Energy and Buildings, 2014.82: p. 675-684.

5.20. Afram, A., Janabi-Sharifi, J., "Theory and applications of HVAC control systems -A review of model predictive control (MPC)", Building and Environment, 2014.72: p. 343-355.

5.21. Zhang, Y., Zhang, T., Wang, R., et al, "Optimal operation of a smart residential microgrid based on model predictive control by considering uncertainties and storage impacts", Solar Energy, 2015.122: p. 1052-1065.

5.22. Liang, W., Quinte, R., Jia, X., Sun, J., "MPC control for improving energy efficiency of a building air handler for multi-zone VAVs", Building and Environment, 2015.92:p.256-268.

5.23. Maasoumy, M., Razmarab, M., Shahbakhtib, M., Vincentelli, A.," Handling model uncertainty in model predictive control for energy efficient buildings", Energy and Buildings, 2014.77: p. 377-392.

5.24. Afram, A., Janabi-Sharifi, J., "Gray-box modeling and validation of residential HVAC system for control system design", Applied Energy, 2015.137: p.134-150.

5.25. Maa, J. Qina, J., Salsbury, T., "Application of economic MPC to the energy and demand minimization of a commercial building", Journal of Process Control, 2014.24: p. 1282-1291.

5.26. Zhaoa, J, Lama, K.,Ydstieb, B., Loftness, V., “Occupant-oriented mixed-mode EnergyPlus predictive control simulation", Energy and Buildings, 2016.117: p. 362-371.

5.27. Chen, X., Wang, Q., Srebric, J., "Occupant feedback based model predictive control for thermal comfort and energy optimization: A chamber experimental evaluation", Applied Energy, 2016.164: p. 341-351.

5.28. Kavgic, M., Hilliard, T., Swan, L., "Opportunities for implementation of MPC in commercial buildings", Energy Procedia, 2015.78 : p. 2148 - 2153

5.29. Zakula, T., Armstrong, P., Norford, L., "Modeling environment for model predictive control of buildings", Energy and Buildings, 2014.85: p. 549-559.

5.30. Xinhua, X., Shengwei, W., Gongsheng, H., "Robust MPC for temperature control of airconditioning systems concerning on constraints and multitype uncertainties", Building Service Engineering, 2010.31: p. 39-55.

5.31. Givoni, b., Reinhold, V., "Climate Considerations in Building and Urban", John Wiley INC, 1998.

5.32. Natural Resources Canada, CanmetENERGY, Varennes (QC) Research Centre, http://www.nrcan.gc.ca/energy/offices-labs/canmet/varennes/5761 (July $2^{\text {nd }} 2016$ ).

5.33. Bhatia, A., "HVAC Made Easy, A Guide to Heating", PDH Center, 2012.

5.34. ASHRAE Fundamentals Handbook, "nonresidential cooling and heating load calculation ", Chapter 28, 1997. 
5.35. Tascikaraoglu, A., Boynuegri, A., Uzunoglu, M., "A demand side management strategy based on forecasting of residential renewable sources: A smart home system in Turkey", Energy and Buildings, 2014.80: p. 309-320.

5.36. Stein, B., "Building technology mechanical and electrical systems", Second edition, John Wiley \& Sons, 1997.

5.37. Zhang, J., Fung, A., Jhingan, S., "Analysis and feasibility study of residential integrated heat and energy recovery ventilator with built-in economizer using an excel spreadsheet program", Energy and Buildings, 2014.75: p. 430-438.

5.38. Sherman M.H. Sherman, "Estimation of infiltration from leakage and climate indicators", Energy and Buildings, 1987.10: p. 81-86.

5.39. Fung, A.S., Guler, B., Aydinalp, M., Ugursal, V.I., "Develop of Canadian Residential Energy End-use and Emission Model", 2000, Halifax, Nova Scotia, CREEDAC.

5.40. Barua, R., assessment and energy benchmarking for two archetype sustainable houses through comprehensive long term monitoring" A thesis presented to Ryerson University, Toronto, Ontario, Canada, 2010.

5.41. Fung, A.S, Zhang, D., "The Archetype Sustainable Houses: Overview of Design and Monitoring Systems", Toronto and Region Conservation's Sustainable Technologies Evaluation Program, June 2011.

5.42. Dembo, A., Fung, A.S., Ng, K.L.R., Pyrka, A., "The archetype sustainable house: investigating its potentials to achieving the net-zero energy status based on the results of a detailed energy audit, in: Proceedings of the 1st International High Performance Buildings Conference", 2010. p. 1-8.

5.43. Safa, A., Fung, A.S, Kumar, R., "Performance of two-stage variable capacity air source heat pump: Field performance results and TRNSYS simulation", Energy and Buildings, 2015.94: p. $80-90$.

5.44. Kumar M, Kar IN, "Non-linear HVAC computations using least square support vector machines", Energy Convers Manag, 2009.50: p. 1411-8.

5.45. Zhang, J., Fung, A.S, "Experimental study and analysis of an energy recovery ventilator and the impacts of defrost cycle", Energy and Buildings, 2015.87: p. 265-271.

5.46. Rupayan, B., "Assessment and energy benchmarking for two archetype sustainable houses through comprehensive long term monitoring", 2010, Master of Applied Science, Ryerson University.

5.47. Kamel, R., Fung, A.S., "Modeling, simulation and feasibility analysis of residential BIPV/T+ASHP system in cold climate—Canada", Energy and Buildings, 2014.82: p. 758770.

5.48. VIESMANN, Technical Data Manual, Gas-fired wall-mounted condensing boiler 12 to 285 MBH (3.5 to $83.5 \mathrm{~kW}$ ), Vitodens 200-W Series B2HA.

5.49. Abedi, S. Alimardani, A., Gharehpetian, G., Riahy G., Hosseinian, S., “A comprehensive method for optimal power management and design of hybrid RES-based autonomous energy systems", Renewable and Sustainable Energy Reviews, 2012.16: p. 1577-1587. 
5.50. Lam, K., Lai, T., Lo, W., To. W., "The application of dynamic modelling techniques to the grid-connected PV (photovoltaic) systems", Energy, 2012.46: p. 264-274.

5.51. Yu, D., "Power Management of a Grid-Connected Distributed Energy Resource System," 2014, Toronto, Canada, Ryerson University.

5.52. Jansuya, P., Kumsuwan, Y., "Design of MATLAB/simulink modeling of fixed-pitch angle wind turbine simulator," Energy Procedia, 2013.34: p.362 - 370 .

5.53. Chung, D., Davidson, C., Fu, R., Ardani, K., Margolis, R., "U.S. Photovoltaic Prices and Cost Breakdowns: Q1 2015 Benchmarks for Residential, Commercial, and Utility-Scale Systems, 2015, U.S. Department of Energy, National Renewable Energy Laboratory.

5.54. Cost of electricity by source, 2016, Wikipedia, https://en.wikipedia.org/wiki/Cost_of_electricity_by_source (July $\left.3^{\text {rd }}, 2016\right)$. 


\section{Chapter 6: Conclusions and Future Works}

\subsection{Conclusions}

The following section represents the contributions of this research work:

- First MPC model (using comprehensive process/plant models):

- Development of novel load shifting and dual fuel switching models as energy conservation strategy planning models (ECSPMs)

- Development of a mechanism for determining the best pre-heating/pre-cooling starting time that results in more energy cost saving in load shifting ECSPM

- Development of simulation framework (Matlab-TRNSYS Co-simulator) to compensate the lack of advanced controllers in building energy simulators and implementing the developed ECSPMs in HVAC system during cooling and heating seasons

- Simulation of the existing house/HVAC energy and control systems

- Second MPC model (using simplified process/plant models):

- Development of an advanced controller empowers the MPC controller to run the HVAC system using different operating time steps in order to reduce the HVAC system energy demand

- Developing an intelligent algorithm (that contains two smart decision support systems) for reducing the MPC controller optimization/computational processing time

- Determination of optimum indoor set point profile (that minimizes HVAC system energy cost) considering outdoor disturbances and TOU pricing scheme

A review was conducted on different control methods in residential HVAC systems including conventional on/off, P/PD/PID, soft/hard, hybrid and model-based predictive control (MPC) controllers. Based on this review, MPC controllers were more effective in controlling HVAC system energy demand and corresponding cost than other methods of control. As a result, the first MPC controller was developed in this research using a Matlab-TRNSYS Co-simulator. This Cosimulator compensated the lack of advanced control mechanisms in building energy simulators (such as TRNSYS, EnergyPlus, and esp-r). After establishing the first MPC controller, a review 
was conducted on the existing energy conservation strategy planning models (ECSPMs) employed in residential house/building HVAC systems. Among different ECSPMs, the focus was more on load shifting (LSH) strategy planning model since this model presents a high level of contribution in managing the load in demand response programs. However, selecting non-optimized preheating/pre-cooling times for load shifting resulted in more HVAC system energy demand during off-peak hours without considerable saving in peak hours and increased the HVAC system energy cost at the end of the simulation day. Hence, a novel LSH-SPM was developed that used a technique for detecting the optimum pre-heating/pre-cooling starting time. This optimum preheating/pre-cooling starting time was detected considering the case study house thermal characteristics, HVAC system properties, and the effects of time-varied outdoor disturbances. In addition to the developed LSH-SPM, a novel dual fuel switching strategy planning model (SDFSSSPM) was also developed. This fuel switching SPM was enabled to calculate the optimum time for switching between natural gas and electricity in order to enhance the capability of the system in managing the demand response. This smart dual fuel switching SPM significantly increased the HVAC system energy cost saving. After developing the load shifting and smart dual fuel switching SPM, an LSHSDFSS strategy planning model was developed as the integration of the two previous models. This mode benefited from the advantages of both LSH and SDFSS models.

Since building energy simulators like TRNSYS suffer from poor control mechanism, they offer no opportunity/mechanism for employing the developed ECSPMs. As a result, a Matlab-TRNSYS Co-simulator was developed. In this co-simulator, a MPC controller was developed using Matlab control platform. A calibrated TRNSYS house model, which was developed by Safa [3.41] for Toronto and Region Conservation Authority (TRCA) Archetype Sustainable House A located in Vaughan, Ontario, Canada, was used and integrated to Matlab MPC controller to investigate the effectiveness of the developed predictive strategy planning models in heating and cooling seasons. Simulation results showed that in the heating season, the operating/energy cost of the HVAC system decreased significantly $(23.8 \%)$ by implementing SDFSS-SPM. LSHSDFSS-SPM reduced the HVAC system operating cost by $15.8 \%$. In the cooling season, LSH-SPM reduced the HVAC system operating cost by $6.63 \%$. Regardless of the capability of the developed MPC controller in implementing ECSPMs in HVAC system, using this MPC for executing comprehensive/complex optimization methods resulted in long computational/optimization time. This happened because of using comprehensive TRNSYS processes/plants models. TRNSYS uses significant computing 
time for running/solving its internal house thermal load balance equations. To solve this problem, the second MPC was developed. In this novel controller, simplified house thermal and HVAC system models were developed in Matlab based on the House A characteristics. These simplified models themselves reduced the MPC computational time since there was no longer a need to run the TRNSYS program. In addition to reducing MPC computational time using simplified models, the developed MPC controller utilized a novel intelligent algorithm to reduce the optimization time. This novel intelligent algorithm consisted of two smart decision support systems (SDSSs). In the first SDSS, an arranged indoor set point profile was generated for each control time horizon using normalization techniques. The first SDSS studied the behavior of the house (HVAC system energy demand) under the effect of time-varied outdoor disturbances during each particular control time horizon and then generated an arranged indoor set point profile (using normalization techniques) which resulted in reducing the HVAC system energy cost in that control time horizon. In the second SDSS, a comprehensive indoor set point matrix was generated taking advantage of TOU pricing scheme. Different control time horizon based probability problems were solved by the second SDSS to generate all useful indoor set point profiles (system states) that resulted in energy cost saving in the HVAC system. The novel intelligent algorithm (a combination of the two SDSSs) empowered the MPC controller in detecting and extracting the most useful scenarios within the defined process space instead of considering all possible scenarios, which in turn resulted in significant reduction in the second MPC optimization/computational time.

Since house thermal demand changes under the effects of time-varied outdoor disturbances, changing the HVAC system operating/control time step may result in HVAC system energy demand reduction. To investigate this, an advanced controller was developed that empowered the second MPC controller to manage the HVAC system operation using different operating time steps. Developing an exhaustive optimization enabled the second MPC controller to detect the optimum HVAC system operating/control time step for each control time horizon.

To increase the energy cost saving, this MPC model was enhanced by integrating on-site renewable energy generation and storage systems.

To show the effectiveness of the developed MPC controller, the energy cost saving after employing MPC controller was compared with base case scenarios (using conventional (on/off) controllers) and a simple rule-based (SRB) controller through the heating season. The simulation results showed that, in the heating season, the novel MPC controller offered $46.71 \%$ and $12.28 \%$ energy 
cost saving with and without using PV/WT/BB systems, respectively, in addition to the simple rule-based control scenario, which itself is considered as an efficient residential HVAC controller. Table 6-1 shows a summary of simulation results on the HVAC system's energy cost saving after employing the first MPC controller (in the heating and cooling seasons) and the second MPC controller in the heating season.

Table 6-1: Summary of simulation results for the first and the second MPC controllers

\begin{tabular}{|c|c|c|c|c|c|c|c|c|c|c|}
\hline MPC Models & \multicolumn{4}{|c|}{ First MPC Controller } & \multicolumn{6}{|c|}{ Second MPC Controller } \\
\hline Description & Energy Conse & rvation st & rategy planr & ning models & Scenarios & \begin{tabular}{|c|} 
First \\
base case \\
scenario \\
$\mathrm{SP}=$ \\
\end{tabular} & $\begin{array}{c}\text { Second } \\
\text { base case } \\
\text { scenario } \\
\mathrm{SP}=22^{\circ} \mathrm{C}\end{array}$ & \begin{tabular}{|c|} 
Third \\
base case \\
scenario \\
$\mathrm{SP}=24^{\circ} \mathrm{C}$ \\
\end{tabular} & \begin{tabular}{|c|} 
Simple \\
rule-based \\
scenario \\
SP=SRB
\end{tabular} & $\begin{array}{c}\text { Simple } \\
\text { rule-based } \\
\text { scenario } \\
\mathrm{SP}=\mathrm{SRB}\end{array}$ \\
\hline ECSPMs & $\begin{array}{c}\text { Load Shifting } \\
\text { (cooling } \\
\text { season) }\end{array}$ & $\begin{array}{c}\text { Load } \\
\text { Shifting }\end{array}$ & $\begin{array}{c}\text { Smart } \\
\text { dual fuel } \\
\text { switching }\end{array}$ & $\begin{array}{c}\text { Load } \\
\text { Shifting and } \\
\text { Smart dual } \\
\text { fuel } \\
\end{array}$ & $\begin{array}{l}\text { Energy } \\
\text { Sources }\end{array}$ & $\begin{array}{c}\mathrm{PV}, \mathrm{WT}, \\
\mathrm{BB} \text { and } \\
\text { local grid }\end{array}$ & $\begin{array}{l}\text { PV,WT, } \\
\text { BB and } \\
\text { local grid }\end{array}$ & $\begin{array}{c}\text { PV,WT, } \\
\text { BB and } \\
\text { local grid }\end{array}$ & $\begin{array}{c}\mathrm{PV}, \mathrm{WT}, \\
\mathrm{BB} \text { and } \\
\text { local grid }\end{array}$ & $\begin{array}{c}\text { only local } \\
\text { grid }\end{array}$ \\
\hline \begin{tabular}{c|} 
Energy \\
Cost \\
Saving (\%) \\
\end{tabular} & 6.63 & -9.11 & 23.8 & 15.8 & \begin{tabular}{|c|} 
Energy \\
Cost \\
Saving (\%) \\
\end{tabular} & 42.46 & 48.46 & 53.23 & 46.71 & 12.28 \\
\hline \begin{tabular}{c|} 
Energy \\
Cost \\
Saving (\$)
\end{tabular} & 14.74 & -88.92 & 232.31 & 154.22 & $\begin{array}{c}\text { Energy } \\
\text { Cost } \\
\text { Saving (\$) }\end{array}$ & 239.71 & 305.49 & 369.83 & 284.74 & 74.93 \\
\hline
\end{tabular}




\subsection{Future Work}

Despite the considerable work on MPC development for controlling HVAC systems, possible research areas that require further investigation/development are summarized as follows:

- Enhancing the second MPC controller process/plant models using gray box modeling techniques. The gray box models take advantage of the qualities of both physics-based and data-driven models; they use physics-based methods for making the model's structure and system performance data to determine the parameters of the model. To be more exact, physics-based models benefit from good generalization capabilities but suffer from insufficient accuracy; whereas, the data-driven models benefit from high accuracy on the training information, but suffer from principle knowledge and physics laws of the system. Gray box models take advantage of good generalization capabilities while compared with data-driven models and benefit from good accuracy while compared with the physics-based models

- Conducting sensitivity analysis to investigate the effects of insulation and thermal mass material on MPC energy cost saving. Since in large cities different materials are used for buildings' structures and buildings can be classified based on their age, a sensitivity analysis can be conducted to measure the productivity of developed MPC controllers on the energy cost saving of different houses/buildings.

- Development of smart learning systems like Fuzzy Logic Learning Systems (FLLS) to optimize MPC performance based on the occupants' behaviors. Since HVAC system consumption is significantly affected by the behavior of occupants, leanings systems that study occupants' behaviors can be integrated to the MPC controller to adjust the indoor set point profile for ahead hours based on the previously recorded activities of occupants.

- Development of Adaptive Calibration Programs for designing simplified MPC process models based on each house characteristics. Using these techniques empowers the MPC controllers to set up and tune their generic house models based on the characteristics and behaviors of each specific house under the effects of outdoor/indoor disturbances.

- Development of new ECSPMs like Away Mode SPM. Integrating the well-developed Away Mode SPM into a MPC controller can significantly reduce HVAC system energy cost by setting appropriate set points during the hours when there is no one in the house. 
Using Levelized Energy Cost instead of Capital Cost Investment for the renewable energy sources. Levelized energy costs include all life-cycle based costs (including fuel, maintenance and capacity factors) associated in producing each unit of energy. 


\section{Appendix I: Intelligent Algorithm Flowchart}

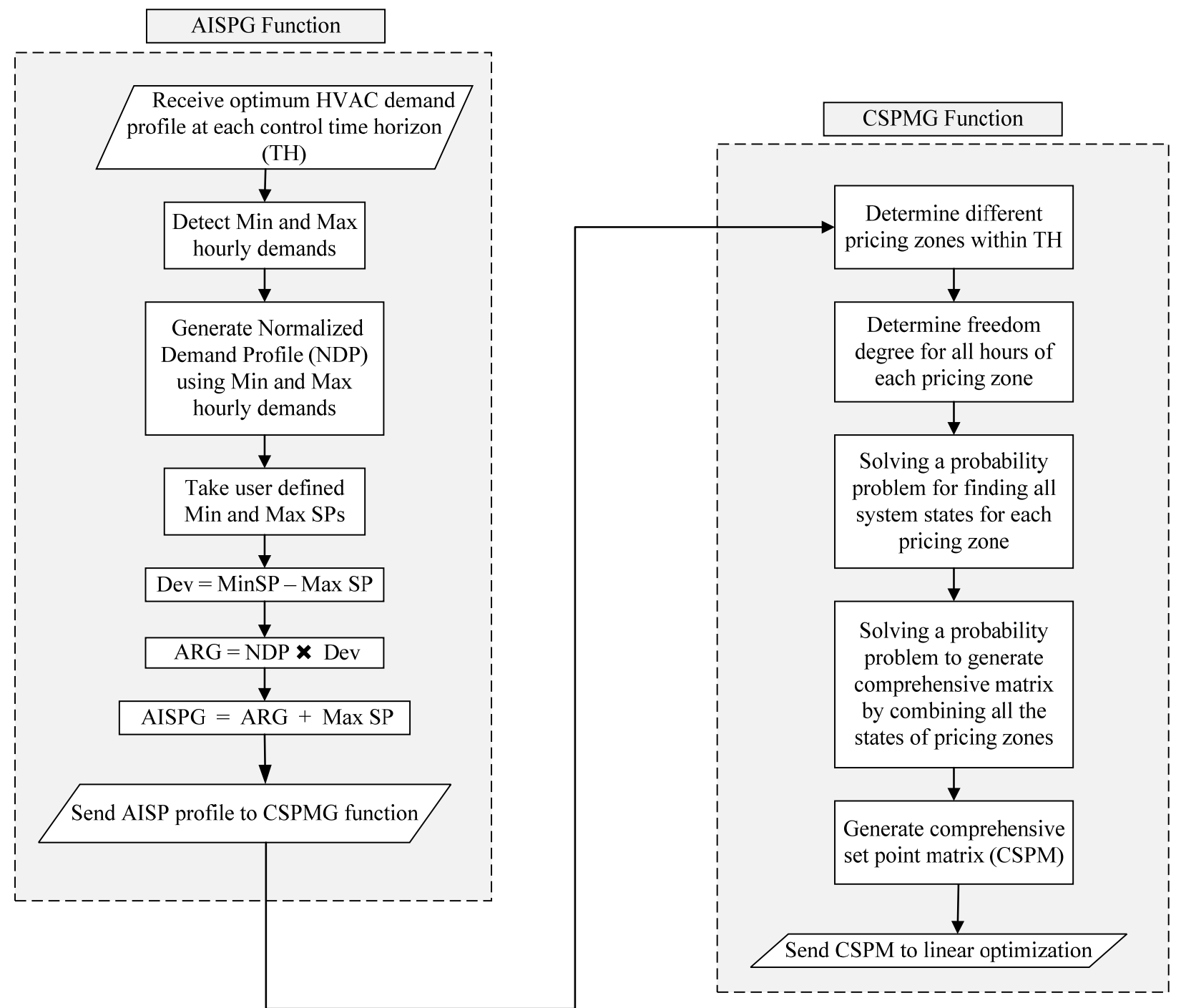

Figure AI-1: Flowchart of intelligent algorithm 
References (Including Chapters 2, 3, 4 and 5)

Chapter 2

2.1. ASHRAE Handbook - Fundamentals, ASHRAE, 2013.

2.2. Sequence of operation for common HVAC system, ASHRAE, 2005.

2.3. Fundamentals of HVAC control systems, ASHRAE, 2011.

2.4. Managing energy and comfort, ASHRAE, 2008.

2.5. Specifying direct digital control systems, ASHRAE, 2005.

2.6. TRNSYS 16 A transient energy simulation program, Mathematical reference: Solar Energy Lab, University of Visconsin-Madison; 2005.

2.7. Kelly, G., Park, C., Clark, D., May, W., "HVACSIM+, A dynamic building/HVAC/control systems simulation program. Proc Workshop HVAC Controls Model Simul”. Seattle, Washington, USA: IBPSA; 1984. p. 175-85.

2.8. EnergyPlus engineering reference: The Reference to EnergyPlus Calculations: DOE; 2013.

2.9. Husaunndee, A., Lahrech, R., Vaezi-Nejad, H., Visier, J., "SIMBAD: A simulation toolbox for the design and test of HVAC control systems", Proc Build Simul. Prague, Czech Republic: IBPSA; 1997. p. 269-76.

2.10. Kalagasidis, AS., Weitzmann, P., Nielsen, TR., Peuhkuri, R., Hagentoft, C-E, Rode, C., “The

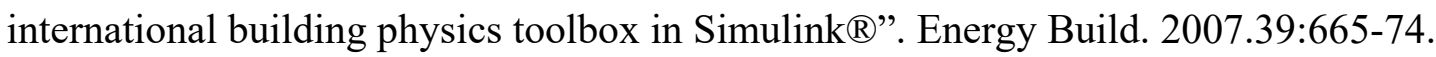

2.11. Kalagasidis, AS., HAM-tools: An integrated simulation tool for heat, air and moisture transfer analyses in building physics [Ph.D Thesis]. Gothenburg, Sweden: Chalmers University of Technology 2004.

2.12. Schijndel, A., Hensen, J., "Integrated heat, air and moisture modeling toolkit in MATLAB ${ }^{\circ}$, Proc 9th Int IBPSA Conf. Montreal, QC, Canada: IBPSA; 2005. p. 1107-11.

2.13. Wemhoner, C., Hafner, B., Schwarzer, K., "Simulation of solar thermal systems with CARNOT blockset in the environment MATLAB® Simulink ${ }^{\circledR ”}$. Proc Eurosun 2000 Conf. Copenhagen, Denmark: ISES; 2000. p. 1-6.

2.14. Mendes, N., Oliveira, G., Araujo, H., Coelho, L., "A MATLAB®-based simulation tool for building thermal performance analysis". Eighth Int IBPSA Conf. Eindhoven, Netherlands: IBPSA; 2003. p. 855-62.

2.15. Novak, PR., Mendes, N, Oliveira, GH., "Simulation of HVAC plants in 2 Brazilian cities

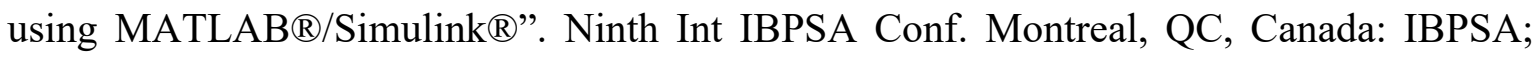
2005. p. 859-66.

2.16. Trcka, M., Hensen, JLM., " Overview of HVAC system simulation”, Autom Constr. 2010;19:93-9.

2.17. Orosa, JA., Oliveira, AC., "Software tools for HVAC research". Adv Eng Software. 2011.42:846-51.

2.18. Riederer, P.," MATLAB ${ }^{\circledR} /$ Simulink ${ }^{\circledR}$ for building and HVAC simulation-State of the art". Ninth Int IBPSA Conf. Montreal, QC, Canada: IBPSA; 2005. p. 1019-26. 
2.19. Wang, S., Ma, Z., "Supervisory and Optimal Control of Building HVAC Systems: A Review", HVAC R Res, 2008, 14 (1): p. 3.

2.20. Naidu, D.S., Craig, G.R., "Advanced control strategies for heating, ventilation, airconditioning, and refrigeration systems - An overview: Part I: Hard control", HVAC R Res, 2011, 17 (1): p. 2-21.

2.21. Afram, Sharifi, "Theory and applications of HVAC control systems. A review of model predictive control (MPC)", Building and Environment, 2014. 72: p 343-355.

2.22. Astrom, K.J., "Theory and applications of adaptive control - A survey", Automatica, 1983. 19 (5): p. 471-486.

2.23. ZlatanoviA, I., et al., "Energy-saving estimation model for hypermarket HVAC systems applications", Energ Buildings, 2011, 43 (12): p. 3353-3359.

2.24. Afram, A., Janabi-Sharifi F., "Review of modeling methods for HVAC systems", Applied Thermal Engineering, 2014, Vol (67), p. 507-519.

2.25. Kalogirou, S.A., "Artificial Neural Networks and Genetic Algorithms in Energy Applications in Buildings". Adv in Build Energ Res, 2009. 3 (1): p. 83-120.

2.26. Jagdev, S., Nirmal, S., "Fuzzy modeling and control of HVAC systems - A review", J of Sci \& Ind Res, 2006. 65: p. 470-476.

2.27. Mirinejad, H., Welch, K.C., Spicer. L., "A review of intelligent control techniques in HVAC systems". IEEE. 2008.

2.28. Dounis, A., Caraiscos, C., "Advanced control systems engineering for energy and comfort management in a building environment - A review", Renew Sust Energ Rev, 2009, 13 (6): p. 1246-1261.

2.29. Bansal, R.C., Pandey, J.C., "Load forecasting using artificial intelligence techniques: a literature survey", Int. J. Computer Applicaitons in Technology, 2005. 22 (2/3): p. 109-119.

2.30. Metaxiotis, K., "Artificial intelligence in short term electric load forecasting: a state-of-theart survey for the researcher", Energy Conversion and Management, 2003.44 (9): p. 15251534.

2.31. Trcka, M., Hensen, J., "Overview of HVAC system simulation”, Autom Constr, 2010.19 (2): p. 93-99.

2.32. Jin, G-Y., Tan, P-Y., Ding, X-D., Koh, T-M., "Cooling coil unit dynamic control of in HVAC system". In: Conf Ind Electron Appl (ICIEA). Beijing, China: IEEE; 2011. p. 942-7.

2.33. Jette, I., Zaheer-uddin, M., Fazio P., "PI-control of dual duct systems: manual tuning and control loop interaction", Energy Convers Manag 1998.39: p. 1471-82.

2.34. Zhang, J., Zhang, K., "A particle swarm optimization approach for optimal design of PID controller for temperature control in HVAC", In: Int Conf Meas Technol Mechatron Autom (ICMTMA). Shanghai, China: IEEE; 2011. p. 230-3.

2.35. Kulkarni, MR., Hong, F., "Energy optimal control of a residential space conditioning system based on sensible heat transfer modeling", Build Environ 2004.39:p.31-8.

2.36. Xu, M., Li, S., Cai, W., "Practical receding-horizon optimization control of the air handling unit in HVAC systems", Ind Eng Chem Res 2005.44: p.2848-55. 
2.37. Zaheer-uddin, M., Tudoroiu, N., "Neuro-PID tracking control of a discharge air temperature system”, Energy Convers Manag 2004.45:p.2405-15.

2.38. Pal, AK., Mudi, RK., " Self-tuning fuzzy PI controller and its applications to HVAC systems". Int J of Comput Cogn 2008.6:p.25-30.

2.39. Soyguder, S., Alli, H. "An expert system for the humidity and temperature control in HVAC systems using ANFIS and optimization with Fuzzy modeling approach". Energy Build 2009.41:p.814-22.

2.40. Lim, D., Rasmussen, BP., Swaroop, D., "Selecting PID control gains for nonlinear HVAC\&R systems", HVAC R Res 2009.15:p.991-1019.

2.41. Wang, Y-G, Shi, Z-G, Cai, W-J. "PID autotuner and its application in HVAC systems. In: Am control Conf. Arlington, Virginia, USA: IEEE. 2001. pp. 2192-6.

2.42. Salsbury, TI., "A survey of control technologies in the building automation industry". In: Proc IFAC 16th world Congr. Prague, Czech Republic 2005. p. 1396.

2.43. Tahersima, F., Stoustrup, J., Rasmussen, H., Nielsen, PG., "Thermal analysis of an HVAC system with TRV controlled hydronic radiator", In: Conf Autom Sci Eng (CASE). Toronto, Ontario, Canada. IEEE; 2010. pp. 756-61.

2.44. Hodgson, DA., "Investigation of a nonlinear controller that combines steady state predictions with integral action [Ph.D thesis]", Fort Collins, Colorado, United States: Colorado State University; 2010.

2.45. Pasgianos, GD., Arvanitis, KG., Polycarpou, P., Sigrimis, N., "A nonlinear feedback technique for greenhouse environmental control", Comput Electron Agric 2003.40:p.15377.

2.46. Hodgson, DA. "Investigation of a nonlinear controller that combines steady state predictions with integral action [Ph.D thesis]", Fort Collins, Colorado, United States: Colorado State University; 2010.

2.47. Anderson, M., Buehner, M., Young, P., Hittle, D., Anderson, C., Jilin, T., et al. "MIMO robust control for HVAC systems". IEEE Tran Control Syst Technol 2008.16:p. 475-83.

2.48. Al-Assadi, SAK, Patel, RV, Zaheer-uddin, M., Verma, MS., Breitinger, J., "Robust decentralized control of HVAC systems using HN-performance measures", J Franklin Inst 2004.341:p.543-67.

2.49. Gouda, MM., Danaher, S., Underwood, CP., "Thermal comfort based fuzzy logic controller". Build Serv Eng Res Technol 2001.22:p.237-53.

2.50. Soyguder, S., Karakose, M., Alli, H., "Design and simulation of self-tuning PID type fuzzy adaptive control for an expert HVAC system", Expert Syst Appl 2009.36:p.4566-73.

2.51. Kusiak, A., Xu, G.," Modeling and optimization of HVAC systems using a dynamic neural network", Energy 2012.42:p.241-50.

2.52. Weiss, MVG., "Adaptive neuro energy management control strategies for HVAC systems in buildings [MASc thesis]". Montreal, Quebec, Canada: Concordia University; 2006. 
2.53. Homod, RZ, Sahari, KSM., Almurib, HAF, Nagi, FH., "Gradient auto-tuned Takagi- Sugeno Fuzzy Forward control of a HVAC system using predicted mean vote index". Energy Build 2012.49:p.254-67.

2.54. Yu, Z, Dexter, A., "Hierarchical fuzzy control of low-energy building systems", Solar Energy 2010.84:p.538-48.

2.55. Liang, J., Du, R., "Thermal comfort control based on neural network for HVAC application", In: Conf control Appl. Toronto, Canada: IEEE; 2005. p. 819-24.

2.56. Henze, GP, Hindman, RE., "Control of air-cooled chiller condenser fans using clustering neural networks", ASHRAE Trans 2002.108:p. 232-44.

2.57. Ben-Nakhi, AE, Mahmoud, MA., "Energy conservation in buildings through efficient A/C control using neural networks". Appl Energy 2002.73:p.5-23.

2.58. Gouda, MM, Danaher, S., "Underwood CP. Quasi-adaptive fuzzy heating control of solar buildings". Build Environ 2006.41:p.1881-91.

2.59. Gregor, P.H., "Experimental Analysis of Model-Based Predictive Optimal Control for Active and Passive Building Thermal Storage Inventory", HVAC\&R Research, 2005, 11 (2): p.189.

2.60. Yuan, S., Perez, R., "Multiple-zone ventilation and temperature control of a single-duct VAV system using model predictive strategy". Energy \& Buildings, 2006, 38 (10): p. 12481261.

2.61. Zhang, D., Barua, R., Fung, A.S., "TRCA-BILD archetype sustainable house-overview of monitoring system and preliminary results for mechanical systems", ASHRAE Trans, 2011, 117 (2) 597-612.

2.62. Crarley, D., Hand, J., Kummert, M., \& Griffith, "Contrasting the Capabilities of Building Energy Perfromance Simulation Programs", 2005, Washington, United States of America.

2.63. Shengwei, W., X. Xinhua, and H., "Gongsheng, Robust MPC for temperature control of airconditioning systems concerning on constraints and multitype uncertainties", Build Serv Eng Res Technol, 2010. 31(1): p. 39-55.

2.64. Ma, J., et al., "Demand reduction in building energy systems based on economic model predictive control". Chemical Engineering Science, 2011, 67 (1): p. 92.

2.65. Cho, S.H., Zaheer-uddin, M., "An experimental study of multiple parameter switching control for radiant floor heating systems". Energy, 1999, 24 (5): p. 433-444.

2.66. Moroşan, P.D., et al., "Building temperature regulation using a distributed model predictive control”. Energy \& Buildings, 2010, 42 (9): p. 1445-1452.

2.67. Li, X, Shi, Z, Hu, S., "A novel control method of a variable volume air conditioning system for indoor thermal environment". In: Int Conf Comput Eng Technol (ICCET). Chengdu, China: IEEE; 2010. p. V2-566-V2-570.

2.68. Salsbury, TI, "A new pulse modulation adaptive controller (PMAC) applied to HVAC systems", Control Eng Pract 2002.10:p.1357-70.

2.69. Seem, JE, "A new pattern recognition adaptive controller with application to HVAC systems", Autom 1998.34:p.969-82. 
2.70. Zaheer-uddin, M, Al-Assadi, SAK, Patel, RV., "Decentralized preview control for multiple disturbance rejection in HVAC systems", Control Eng Pract 1994.2:p.989-1000.

2.71. Morosan, P-D, Bourdais, R., Dumur, D., Buisson, J., "Building temperature regulation using a distributed model predictive control", Energy Build 2010.42:p. 1445-52.

2.72. Freire, Z., Oliveira, G., Mendes, N., "Predictive controllers for thermal comfort optimization and energy savings", Energy and Buildings, 2008.40: p.1353-1356.

2.73. Huang, G., "Model predictive control of VAV zone thermal systems concerning bi-linearity and gain nonlinearity". Control Eng Pract, 2011.19 (7): p. 700-710.

2.74. Karlsson, H., "Application of model based predictive control for water-based floor heating in low energy residential buildings", Building and Environment, 2011, 46 (3): p. 556-569.

2.75. Candanedo, J., Athienitis, A., "Predictive control of radiant floor heating and solar-source heat pump operation in a solar house", HVAC\&R Research, 2011, 17 (3): p. 235-256.

2.76. Li, J., "Dynamic zone modeling for HVAC system control". Int J of Model, Identif and Control, 2010. 9 (1/2): p. 5-13.

2.77. Ma, J., Qin, J., Salsbury, T., Xu, P., "Demand reduction in building energy systems based on economic model predictive control". Chem Eng Sci 2011.67:p.92-100.

2.78. Huang, G., "Model predictive control of VAV zone thermal systems concerning bi-linearity and gain nonlinearity". Control Eng Pract 2011.19:p.700-10.

2.79. Rehrl, J., Horn, M., "Temperature control for HVAC systems based on exact linearization and model predictive control". In: Int Conf Control Appl (CCA). Denver, Colorado, USA: IEEE; 2011. pp. 1119-24.

2.80. Karlsson, H., Hagentoft, C-E., "Application of model based predictive control for waterbased floor heating in low energy residential buildings", Build Environ 2011.46:p.556-69.

2.81. Henze, GP, Kalz, DE, Liu, S, Felsmann C, "Experimental analysis of model-based predictive optimal control for active and passive building thermal storage inventory", HVAC R Res 2005.11:p.189-213.

2.82. Henze, GP, Dodier, RH, Krarti, M., "Development of a predictive optimal controller for thermal energy storage systems". HVAC R Res 1997.3:p.233-64.

2.83. Balan, R., Cooper, J., Chao, K-M, "Stan S, Donca R. Parameter identification and model based predictive control of temperature inside a house", Energy Build 2011.43:p.748-58.

2.84. Siroky J, Oldewurtel F, Cigler J, Privara S. Experimental analysis of model predictive control for an energy efficient building heating system. Appl Energy 2011.88:3079-87.

2.85. Privara, S., Siroky, J., Ferkl, L., Cigler, J., "Model predictive control of a building heating system: the first experience", Energy Build 2011.43:p.564-72.

2.86. Ferkl, L., Siroky, J., "Ceiling radiant cooling: comparison of ARMAX and subspace identification modelling methods", Build Environ 2010.45:p.205-12.

2.87. Wallace, M., et al., "Energy efficient model predictive building temperature control", Chemical Engineering Science, 2012, 69 (1): p. 45-58. 
2.88. Oldewurtel, F., Parisio, A., Jones, CN, Gyalistras, D., Gwerder, M., Stauch, V., et al., "Use of model predictive control and weather forecasts for energy efficient building climate control”, Energy Build 2012.45:p.15-27.

2.89. Elliott, MS. "Decentralized model predictive control of a multiple evaporator HVAC system [MSc thesis]”. College Station, Texas, United States: Texas A\&M University; 2008.

2.90. Xu, M., Li, S. "Practical generalized predictive control with decentralized identification approach to HVAC systems", Energy Convers Manag 2007.48:p.292-9.

2.91. Tazvinga, H., Zhu, B., Xia, X., "Energy dispatch strategy for a photovoltaic-wind-dieselbattery hybrid power system", Solar Energy, 2014.108:p. 412-420.

2.92. Beghi, A., Cecchinato, L., Paggiaro, F., Rampazzo, M., "VAVAC systems modeling and simulation for FDD applications", In: Int Conf Control Autom (ICCA). Santiago, Chile: IEEE; 2011. pp. 800-5.

2.93. Atthajariyakul, S., Leephakpreeda, T., "Real-time determination of optimal indoor-air condition for thermal comfort, air quality and efficient energy usage", Energy Build 2004.36:p.720-33.

2.94. Tashtoush, B., Molhim, M., Al-Rousan, M., "Dynamic model of an HVAC system for control analysis”, Energy 2005.30:p.1729-45.

2.95. Wemhoff, AP, Frank, MV. "Predictions of energy savings in HVAC systems by lumped models",Energy Build 2010.42:p.1807-14.

2.96. Thosar, A., Patra, A., Bhattacharyya, S. "Feedback linearization based control of a variable air volume air conditioning system for cooling applications", ISA Trans 2008.47:p.339-49.

2.97. Vasak, M., Starcic, A., Martincevic, A., "Model predictive control of heating and cooling in a family house", In: Proc 34th Int convention MIPRO. Opatija, Croatia: IEEE; 2011. pp. 739-43.

2.98. Homod, RZ, Sahari, KSM, Almurib, HAF, Nagi, FH. "RLF and TS fuzzy model identification of indoor thermal comfort based on PMV/PPD”. Build Environ 2012.49:p.141-53.

2.99. Soyguder, S., Alli, H., "Predicting of fan speed for energy saving in HVAC system based on adaptive network based fuzzy inference system”, Expert Syst Appl 2009.36:p.8631-8.

2.100. Chen, J., Lian, Z., Tan, L., Zhu, W., Zhang, W., "Modeling and experimental research on ground-source heat pump in operation by neural network", In: Int Conf Comput Distrib Control Intell Environ Monit CDCIEM. Changsha, China: IEEE; 2011. pp. 459-62.

2.101. Ding, L., Lv, J., Li, X., Li, L., "Support vector regression and ant colony optimization for HVAC cooling load prediction", In: Int Symp Comput Commun Control Autom (3CA). Tainan, Taiwan: IEEE; 2010. pp. 537-41.

2.102. Wang, S., Xu, X., Huang, G., "Robust MPC for temperature control of air conditioning systems concerning on constraints and multitype uncertainties", Build Serv Eng Res Technol 2010.31:p.39-55.

2.103. Bi, Q., Cai, W-J, Wang, Q-G, Hang, C-C, Lee, E-L, Sun, Y, et al. “Advanced controller auto-tuning and its application in HVAC systems”, Control Eng Pract 2000.8: p.633-44. 
2.104. Yiu, CMJ. "Statistical modelling and forecasting schemes for air-conditioning system $[\mathrm{Ph} . \mathrm{D}$ thesis]", Hung Hom, Kowloon, Hong Kong: The Hong Kong Polytechnic University; 2008.

2.105. Matlab. Signal smoothing. Signal processing toolbox: MathWorks; 2013.

2.106. Tang, F. "HVAC system modeling and optimization: a data-mining approach [MSc thesis]". Iowa City, Iowa, United States: The University of Iowa; 2010.

2.107. Energy estimating and modeling methods. ASHRAE handbook fundamentals. ASHRAE; 2009.

2.108. Kusiak, A., Li, M., Zhang, Z., "A data-driven approach for steam load prediction in buildings", Appl Energy 2010.87:p.925-33.

2.109. Kumar, M., Kar, IN. "Non-linear HVAC computations using least square support vector machines", Energy Convers Manag 2009.50:p.1411-8.

2.110. Wu, S., Sun, J-Q. "A physics-based linear parametric model of room temperature in office buildings". Build Environ 2012.50:p.19.

2.111. Maasoumy, MH. "Modeling and optimal control algorithm design for HVAC systems in energy efficient buildings [MSc thesis]". Berkeley, California, United States: University of California at Berkeley; 2011.

2.112. Candanedo, JA, Athienitis, AK., "Predictive control of radiant floor heating and solarsource heat pump operation in a solar house", HVAC R Res 2011.17:p. 235-56.

2.113. Wang, S., Ma, Z., "Supervisory and optimal control of building HVAC systems: a review", HVAC R Res 2008.14:p.3-32.

2.114. Ahmad, M.W., Eftekhari, M., "Investigating the performance of a combined solar system with heat pump for houses", Energy and Buildings 2013.63:p. 138-146.

2.115. Rehrl, J., Horn. M., "Temperature control for HVAC systems based on exact linearization and model predictive control", International Conference on Control Applications (CCA). 2011, Denver, CO, USA: IEEE.

2.116. Gregor, P., Henze, R.H.D., Moncef, K., "Development of a Predictive Optimal Controller for Thermal Energy Storage Systems", HVAC\&R Research, 1997.3 (3): p. 233-264.

2.117. Elliott, M.S., "Decentralized model predictive control of a multiple evaporator HVAC system". 2008.

2.118. Xu, M., Li, S., Cai, W., "Practical Receding-Horizon Optimization Control of the Air Handling Unit in HVAC Systems". Industrial \& Engineering Chemistry Research, 2005, 44 (8): p. 2848-2855.

2.119. Platt, G., Ward, J., Wall, J., “Optimal supervisory HVAC control: experiences in Australia”. HVAC R Res 2011.17:p.297-308.

2.120. Canbay, CS. "Optimization of HVAC control strategies by building management systems case study: Ozdilek shopping center [MSc thesis]". Izmir, Turkey: Izmir Institute of Technology; 2003.

2.121.Naidu,DS, Rieger, CG., "Advanced control strategies for heating, ventilation, air conditioning, and refrigeration systems e an overview: part I: hard control." HVAC R Res 2011.17:p.2-21. 
2.122. Ferkl, L., Siroky, J. "Ceiling radiant cooling: comparison of ARMAX and subspace identification modelling methods". Build Environ 2010.45:p.205-12.

2.123. Zhou, G., Krarti, M., Henze, GP, "Parametric analysis of active and passive building thermal storage utilization'. J Sol Energy Eng 2005.127:p.37-46.

Chapter 3

3.1. Mathew, V., Sitaraman, R.K., Shenoy, P., "Reducing energy costs in Internet-scale distributed systems using load shifting", Communication Systems and Networks (COMSNETS), Sixth International Conference, Bangalore, 2014. p. $1-8$.

3.2. Liu, Y., Yuen, C., Member, S., "Peak-to-Average Ratio Constrained Demand-Side Management With Consumer's Preference in Residential Smart Grid", Selected Topics in Signal Processing, IEEE Journal, 2014.8(6): p. 1084 - 1097.

3.3. Radhakrishnan, A, Selvan, M.P., "Load scheduling for smart energy management in residential buildings with renewable sources", Power Systems Conference (NPSC), 2014 Eighteenth National, Guwahati, 2014. p. $1-6$

3.4. Afram, A, Janabi-Sharifi, F, "Gray-box modeling and validation of residential HVAC system for control system design", Applied Energy, 2015. (137): p. 134-150.

3.5. Ma, J, Qin, J, Salsbury, T, Xu, P, "Demand reduction in building energy systems based on economic model predictive control", Chemical Engineering Science, 2011. 67(1): p. 92

3.6. Candanedo, J, Athienitis, A, "Predictive control of radiant floor heating and solar-source heat pump operation in a solar house", HVAC\&R Research, 2011. 17(3): p. 235-256.

3.7. Naidu, D.S, Craig, G.R, "Advanced control strategies for heating, ventilation, airconditioning, and refrigeration systems - An overview: Part I" ,Hard control. HVAC R Res, 2011. 17(1): p. 2-21.

3.8. Weiss, M.V.G., "Adaptive neuro energy management control strategies for HVAC systems in buildings, in Build", Civ and Environ Eng. 2006, Concordia University: Montreal, Quebec, Canada.

3.9. Platt, G, Ward, J, Wall, J, "Optimal supervisory HVAC control: Experiences in Australia", HVAC R Res, 2011. 17(3): p. 297-308.

3.10. Srinivas, K, Ning, L, "Evaluation of Residential HVAC Control Strategies for Demand Response Programs", ASHRAE Trans, 2006. 112: p. 535-546.

3.11. García-Domingo, B., Torres-Ramírez, M., Casa, J., Aguilera, J., "Design of the back-up system in Patio 2.12 photovoltaic installation”, Energy and Buildings, 2014. 83: P 130-139.

3.12. Keshtkar, A, Arzanpour, S, Ahmadi, P, "Smart residential load reduction via fuzzy logic, wireless sensors, and smart grid incentives", Energy and Buildings, 2015. 104: p 165-180.

3.13. Onda, H., Yamamoto, S., Takeshit, H., Okamoto, S., "Peak Load Shifting and Electricity Charges Reduction Realized by Electric Vehicle Storage Virtualization", AASRI Procedia, 2014. 7: p 101-106.

3.14. Boehm, R.F., "An approach to decreasing the peak electrical demand in residences", Energy Procedia, 2012. 14: p 337-342. 
3.15. Castillo-Cagigal, M., Gutierrez, A., Monastrieo, F., Caamano, E., Masa, D., "A semidistributed electric demand-side management system with PV generation for selfconsumption enhancement", Energy Conversion and Management, 2011. 7: p 2659-2666.

3.16. Fernandes, F., Morais, H., Vale, Z.," Dynamic load management in a smart home to participate in demand response events", Energy and Buildings, 2014. 82: p 592-606.

3.17. Beizaee, A, Allison, D., Lomas, K., Foda, E., Loveday, D., "Measuring the potential of zonal space heating controls to reduce energy use in UK homes: The case of un-furbished 1930s dwellings", Energy and Buildings, 2015. 92: p 29-44.

3.18. H.F. Naspolini, H.S.G. Militão, R. Rüther, "The role and benefits of solar water heating in the energy demands of low-income dwellings in Brazil", Energy Conversion and Management, 2010. 51: p 2835-2845.

3.19. Chassin, DP., Stoustrup, J., Agothoklis, P., Djilali, N., "A new thermostat for real-time price demand response: Cost, comfort and energy impacts of discrete-time control without deadband", Applied Energy, 2015. 155: p 816-825.

3.20. Li, J., Poulton, G., Platt, G., Wall, j., James, G., “ Dynamic zone modelling for HVAC system control”, Int J of Model, Identif and Control, 2010. 9(1/2): p. 5-13.

3.21. Nielsen, T.R., Drivsholm, C., "Energy efficient demand controlled ventilation in single family houses", Energy and Buildings, 2010. 42: p 1995-1998.

3.22. Gulden, C.C.S.G., "Optimization of HVAC control strategies by building management systems case study: Özdilek shopping center in Energy Engineering” ,. 2003, Izmir Institute of Technology: Izmir, Turkey.

3.23. Hart, R., "Advanced unitary HVAC control sequence", ASHRAE Transactions, 2012. 118(1): p. 628.

3.24. Reid, H., Callahan, J., Anderson, K., Johanning, P., "Unitary HVAC Premium Ventilation Upgrade", ASHRAE Transactions, 2011. 117(1): p. 517.

3.25. Al-Rabghi, O.M. and M.M. Akyurt, "A survey of energy efficient strategies for effective air conditioning", Energy Conversion and Management, 2004. 45(11): p. 1643-1654.

3.26. Pal, A.K. and R.K. Mudi, "Self-Tuning Fuzzy PI Controller and its Application to HVAC Systems", Int. J. Computer Cognition, 2008. 6(1): p. 25-30.

3.27. Bansal, R.C. and J.C. Pandey, "Load forecasting using artificial intelligence techniques: a literature survey" Int. J. Computer Applications in Technology, 2005. 22(2/3): p. 109-119.

3.28. Kulkarni, M.R. and F. Hong, "Energy optimal control of a residential space-conditioning system based on sensible heat transfer modeling", Building and Environment, 2004. 39: p. 31-38.

3.29. Ouden, C., "Buildings thermal analysis, an electronic mathcad book: A.K. Athienitis, MathSoft, Cambridge, USA", Solar Energy, 1997. 60: p 61.

3.30. Wills, A., Cynthia A. Cruickshank, Ian Beausoleil-Morrison," Application of the ESPr/TRNSYS Co-Simulator to Study Solar Heating with a Single-House Scale Seasonal Storage", Energy Procedia, 2012. 30: p 715-722. 
3.31. Justin C. DeBlois, Melissa M. Bilec, Laura A. Schaefer," Design and zonal building energy modeling of a roof integrated solar chimney", Renewable Energy, 2013. 52: p 241-250.

3.32. Afram, A., Janabi-Sharifi, F., "Theory and application of HVAC control systems - A review of model predictive control (MPC)", Building and Environment, 2014. 72: p. 343-355.

3.33. http://dd.weatheroffice.ec.gc.ca. (Jan 4th, 2015).

3.34. Oldewurtela, F., Parisio, A., Jones, C., Gyalistras, D., Stauch, V., "Use of model predictive control and weather forecasts for energy efficient building climate control", Energy and Buildings, 2012. 45: p. 15-27

3.35. Poulin, L., "Weather Forecast Data An Important Input into Building Management Systems", National Prediction Operations Division, ICEBO 2013, Montreal, Qc. October, 2013.

3.36. Kim, S., Augenbroe, G., "Using the National Digital Forecast Database for model-based building controls", Automation in Construction, 2012. 27: p. 170-182

3.37. Poulin, L., "An NWP forecast information matrix in support of renewable energy applications", CMOS 2013, Saskatoon

3.38. Vernaya, C., Pitavala, S., Blancb, P. "Review of satellite-based surface solar irradiation databases for the engineering, the financing and the operating of photovoltaic system", Energy Procedia, 2014. 57: p. 1383 - 1391.

3.39. Rodriguez, A., antonio, J., Pozo, D., Tovar, J., “An artificial neural network ensemble model for estimating global solar radiation from Meteosat satellite images", Energy, 2013. 61: p. 636- 645 .

3.40. Fung, A., Zhang, D., "The Archetype Sustainable Houses: Overview of Design and Monitoring Systems", Toronto and Region Conservation's Sustainable Technologies Evaluation Program, June 2011.

3.41. Safa, A., Fung, A., Kumar, R., "Performance of two-stage variable capacity air source heat pump: Field performance results and TRNSYS simulation", Energy and Buildings, 2015. 94: p. 80-90.

3.42. Dembo, A., Fung, A.S., Ng, K.L.R., Pyrka, A., "The archetype sustainable house: investigating its potentials to achieving the net-zero energy status based on the results of a detailed energy audit, in: Proceedings of the 1st International High Performance Buildings Conference", 2010. p. 1-8.

3.43. Zhang, D., Barua, R., Fung, A.S., “ TRCA-BILD archetype sustainable house-overview of monitoring system and preliminary results for mechanical systems, ASHRAE Trans, 2011. 117 (2): p. 597-612.

3.44. VIESMANN, Technical Data Manual, Gas-fired wall-mounted condensing boiler 12 to 285 MBH (3.5 to $83.5 \mathrm{~kW})$, Vitodens 200-W Series B2HA.

3.45. https://en.wikipedia.org/wiki/Geography_of_Toronto. (Oct 6th, 2015).

3.46. https://en.wikipedia.org/wiki/List_of_extreme_temperatures_in_Canada. (Oct 6th, 2015).

3.47. ASHRAE 2009, ASHRAE Handbook-Fundamentals, American Society of Heating, Refrigeration and Air-Conditioning Engineers, Inc., Atlanta, GA. 
3.48. Crarley, D., Hand, J., Kummert, M., \& Griffith, " Contrasting the Capabilities of Building Energy Perfromance Simulation Programs", 2005, Washington, United States of America.

3.49. Klein, S., Beckman, W., Mitchell, J., Duffie, J., Duffie, N., Freeman, T., "TRNSYS 16 - A Transient System Simulation Program", 2006, Madison, Wisconsin, U.S.A.

3.50. Kamel, R., Fung, A.S., "Modeling, simulation and feasibility analysis of residential BIPV/T+ASHP system in cold climate—Canada", Energy and Buildings, 2014. 82: p. 758770 .

\section{Chapter 4}

4.1. Xu, X., Taylor, J., Pisello, A., "Network synergy effect: Establishing a synergy between building network and peer network energy conservation effects", Energy and Buildings, 2014. 68: p. 312-320.

4.2. Xu, X., Taylor, J., Pisello, A., Culliga, P., "The impact of place-based affiliation networks on energy conservation: An holistic model that integrates the influence of buildings, residents and the neighborhood context", Energy and Buildings, 2012. 55: p. 637-646.

4.3. Pagani, G., Aiello, M., "The Power Grid as a complex network: A survey", The Electricity Journal, 2013. 26(6): p. 74-78.

4.4. Siano, P., Sarno, D., "Assessing the benefits of residential demand response in a real time distribution energy market”, Applied Energy, 2016. 161: p. 533-551.

4.5. Arteconi A., Ciarrocchi, E., Pan, Q., Carducci, F., "Thermal energy storage coupled with PV panels for demand side management of industrial building cooling loads", Applied Energy (2016)

4.6. Vakiloroaya, V., Somali, B., Fakhar, A., Pishghadam, K., “A review of different strategies for HVAC energy saving", Energy Conversion and Management, 2014. 77: P. 738-754.

4.7. Huang, H., Chen, L., Hu, E., "A new model predictive control scheme for energy and cost savings in commercial buildings: An airport terminal building case study", Building and Environment, 2015. 89: P. 203-216.

4.8. Ghahramani A., Zhang, K., Dutta, K., Yang, Z., Gerber, B., "Energy savings from temperature setpoints and deadband: Quantifying the influence of building and system properties on savings", Applied Energy, 2016. 165: P. 930-942.

4.9. Kim, W., Jeon, Y., Kim, Y., "Simulation-based optimization of an integrated daylighting and HVAC system using the design of experiments method", Applied Energy, 2016. 162: P. 666-674.

4.10. Nazi, W., Wang, Y., Roskilly, T., "Methodologies to Reduce Cooling Load using Heat Balance Analysis: A Case Study in an Office Building in a Tropical Country", Energy Procedia, 2015. 75: P.1269 - 1274.

4.11. Christantonia, D., Flynna, D., Finnb, D., "Modelling of a Multi-purpose Commercial Building for Demand Response Analysis”, Energy Procedia, 2015. 78: P. 2166 - 2171. 
4.12. Kramer, R., Maas, M., Martens, M., Schijndel, A., Schellen, H., "Energy conservation in museums using different setpoint strategies: A case study for a state-of-the-art museum using building simulations", Applied Energy, 2015. 158: P. 446-458.

4.13. Wang, Q., Augenbroe,G.,Kim, J., Gu, L., "Meta-modeling of occupancy variables and analysis of their impact on energy outcomes of office buildings", Applied Energy, 2016. 174: P. $166-180$

4.14. Patteeuw, D., Gregor, P., Helsen, L., "Comparison of load shifting incentives for low-energy buildings with heat pumps to attain grid flexibility benefits", Applied Energy, 2016. 167: P. 80-92.

4.15. Beizaee, A, Allison, D., Lomas, K., Foda, E., Loveday, D., "Measuring the potential of zonal space heating controls to reduce energy use in UK homes: The case of un-furbished 1930s dwellings", Energy and Buildings, 2015. 92: p 29-44.

4.16. Chassin, DP., Stoustrup, J., Agothoklis, P., Djilali, N., "A new thermostat for real-time price demand response: Cost, comfort and energy impacts of discrete-time control without deadband", Applied Energy, 2015. 155: p. 816-825.

4.17. Li, J., Poulton, G., Platt, G., Wall, j., James, G., "Dynamic zone modelling for HVAC system control", Int J of Model, Identif and Control, 2010. 9(1/2): p. 5-13.

4.18. Radhakrishnan, N., Su, Y., Su, R., Poolla, K., "Token based scheduling for energy management in building HVAC systems", Applied Energy, 2016. 173: P. 67-79.

4.19. Griful, S., Jacobsen, R., Nguyen, D., Sorensen, G., "Demand response potential of ventilation systems in residential buildings", Energy and Buildings, 2016. 121: P. 1-10.

4.20. Di Giorgio, A., Liberati, F., "Near real time load shifting control for residential electricity prosumers under designed and market indexed pricing models", Applied Energy, 2014. 128: p. 119-132.

4.21. Kamel, R., Fung, A.S., "Modeling, simulation and feasibility analysis of residential BIPV/T+ASHP system in cold climate-Canada", Energy and Buildings, 2014. 82: p. 758770.

4.22. ASHRAE 2009, ASHRAE Handbook-Fundamentals, American Society of heating, Refrigeration and Air-Conditioning Engineers, Inc., Atlanta, GA

4.23. Zhang, D., Barua, R., Fung, A., "TRCA-BILD Archetype Sustainable House - Overview of Monitoring System and Preliminary Results for Mechanical Systems, ASHRAE Transactions, 2011: p.597-612.

4.24. Safa, A., Fung, A., Kumar, R., "Performance of two-stage variable capacity air source heat pump: Field performance results and TRNSYS simulation", Energy and Buildings, 2015. 94: p. 80-90. 
Chapter 5

5.1. L. Prez-Lombard, J. Ortiz, and C. Pout, “A review on buildings energy consumption information," Energy and Buildings, 2008.40(3): p.394-398.

5.2. Huang, H., Chen, L., Hu, E., " A new model predictive control scheme for energy and cost savings in commercial buildings: An airport terminal building case study", Building and Environment, 89 (2015.89: p. 203-216.

5.3. Afram, A., Janabi-Sharifi, Gray-box modeling and validation of residential HVAC system for control system design, Applied Energy, 2015.137: p. 134-150

5.4. Freire, Z., Oliveira, G., Mendes, N., "Predictive controllers for thermal comfort optimization and energy savings, Energy and Buildings, 2008.40:p. 1353-1356.

5.5. Afram, A., Janabi-Sharifi, F., F., Theory and application of HVAC control systems - A review of model predictive control (MPC), Building and Environment, 2014.72: p. 343355.

5.6. Shengwei, W., X. Xinhua, and H., Gongsheng, Robust MPC for temperature control of airconditioning systems concerning on constraints and multitype uncertainties. Build Serv Eng Res Technol, 2010. 31(1): p. 39-55.

5.7. Ma, J., Qin, J., Salsbury, T., Xu, P., "Demand reduction in building energy systems based on economic model predictive control. Chemical Engineering Science, 2011, 67 (1): p. 92 100.

5.8. Prívara, S., Siroky, J., Ferk1, L., Cigler, J., "Model predictive control of a building heating system: The first experience. Energy \& Buildings, 2011, 43 (2): p. 564-572.

5.9. Moroşan, P.D., Bourdais, B., Dumur, D., Buisson, J., "Building temperature regulation using a distributed model predictive control”, Energy \& Buildings, 2010, 42 (9): p. 1445 1452

5.10. Huang, H., Chen, 1., Hu, E., "new model predictive control scheme for energy and cost savings in commercial buildings: An airport terminal building case study', Building and Environment, 2015.89: p. 203-216

5.11. Hilliarda, C., Swana, L., Kavgica, M., et al., "Development of a whole building model predictive control strategy for a LEED silver community college”, Energy and Buildings, 2016.111: p. 224-232

5.12. Bruni, G., Cordiner, G, Mulone, V. Sinisi,V., "Energy management in a domestic microgrid by means of model predictive controllers", Energy 2016.108: p. 119-131.

5.13. Razmara, M., Maasoumy M., Shahbakhti, M., Robinett, RD., "Optimal exergy control of building HVAC system”, Applied Energy, 2015.156: p. 555-565.

5.14. Lee, Y., Horesha, R., Libertib, L., "Optimal HVAC control as demand response with onsite energy storage and generation system”, Energy Procedia, 2015.78: p. $2106-2111$

5.15. Ascionea, C., Biancoa, N., Stasioa, C., et al., "Simulation-based model predictive control by the multi-objective optimization of building energy performance and thermal comfort", Energy and Buildings, 2016.111: p.131-144. 
5.16. Mirakhorli, A., Dong, B., "Occupancy behavior based model predictive control for building indoor climate-A critical review", Energy and Buildings, 2016.129: p. 499-513.

5.17. Schibuola, L., Scarpa, M., Tambani, C., "Demand response management by means of heat pumps controlled via real time pricing", Energy and Buildings, 2015.90: p. 15-28

5.18. West, S., Ward, J., Wall, J., "Trial results from a model predictive control and optimization system for commercial building HVAC", Energy and Buildings, 2014.72: p. 271-279.

5.19. Dobbs, J., Hencey B., "Model predictive HVAC control with online occupancy model", Energy and Buildings, 2014.82: p. 675-684.

5.20. Afram, A., Janabi-Sharifi, J., "Theory and applications of HVAC control systems -A review of model predictive control (MPC)", Building and Environment, 2014.72: p. 343355.

5.21. Zhang, Y., Zhang, T., Wang, R., et al, "Optimal operation of a smart residential microgrid based on model predictive control by considering uncertainties and storage impacts", Solar Energy, 2015.122: p. 1052-1065.

5.22. Liang, W., Quinte, R., Jia, X., Sun, J., "MPC control for improving energy efficiency of a building air handler for multi-zone VAVs", Building and Environment, 2015.92:p.256268.

5.23. Maasoumy, M., Razmarab, M., Shahbakhtib, M., Vincentelli, A.," Handling model uncertainty in model predictive control for energy efficient buildings", Energy and Buildings, 2014.77: p. 377-392.

5.24. Afram, A., Janabi-Sharifi, J., "Gray-box modeling and validation of residential HVAC system for control system design", Applied Energy, 2015.137: p.134-150.

5.25. Maa, J. Qina, J., Salsbury, T., "Application of economic MPC to the energy and demand minimization of a commercial building", Journal of Process Control, 2014.24: p. 12821291.

5.26. Zhaoa, J, Lama, K.,Ydstieb, B., Loftness, V., “'Occupant-oriented mixed-mode EnergyPlus predictive control simulation", Energy and Buildings, 2016.117: p. 362-371.

5.27. Chen, X., Wang, Q., Srebric, J., "Occupant feedback based model predictive control for thermal comfort and energy optimization: A chamber experimental evaluation", Applied Energy, 2016.164: p. 341-351.

5.28. Kavgic, M., Hilliard, T., Swan, L., "Opportunities for implementation of MPC in commercial buildings", Energy Procedia, 2015.78 : p. 2148 - 2153

5.29. Zakula, T., Armstrong, P., Norford, L., "Modeling environment for model predictive control of buildings", Energy and Buildings, 2014.85: p. 549-559.

5.30. Xinhua, X., Shengwei, W., Gongsheng, H., "Robust MPC for temperature control of airconditioning systems concerning on constraints and multitype uncertainties", Building Service Engineering, 2010.31: p. 39-55.

5.31. Givoni, b., Reinhold, V., "Climate Considerations in Building and Urban", John Wiley INC, 1998. 
5.32. Natural Resources Canada, CanmetENERGY, Varennes (QC) Research Centre, http://www.nrcan.gc.ca/energy/offices-labs/canmet/varennes/5761 (July $\left.2^{\text {nd }} 2016\right)$.

5.33. Bhatia, A., "HVAC Made Easy, A Guide to Heating", PDH Center, 2012.

5.34. ASHRAE Fundamentals Handbook, "nonresidential cooling and heating load calculation ", Chapter 28, 1997.

5.35. Tascikaraoglu, A., Boynuegri, A., Uzunoglu, M., "A demand side management strategy based on forecasting of residential renewable sources: A smart home system in Turkey", Energy and Buildings, 2014.80: p. 309-320.

5.36. Stein, B., "Building technology mechanical and electrical systems", Second edition, John Wiley \& Sons, 1997.

5.37. Zhang, J., Fung, A., Jhingan, S., "Analysis and feasibility study of residential integrated heat and energy recovery ventilator with built-in economizer using an excel spreadsheet program", Energy and Buildings, 2014.75: p. 430-438.

5.38. Sherman M.H. Sherman, "Estimation of infiltration from leakage and climate indicators", Energy and Buildings, 1987.10: p. 81-86.

5.39. Fung, A.S., Guler, B., Aydinalp, M., Ugursal, V.I., "Develop of Canadian Residential Energy End-use and Emission Model", 2000, Halifax, Nova Scotia, CREEDAC.

5.40. Barua, R., assessment and energy benchmarking for two archetype sustainable houses through comprehensive long term monitoring" A thesis presented to Ryerson University, Toronto, Ontario, Canada, 2010.

5.41. Fung, A.S, Zhang, D., "The Archetype Sustainable Houses: Overview of Design and Monitoring Systems", Toronto and Region Conservation's Sustainable Technologies Evaluation Program, June 2011.

5.42. Dembo, A., Fung, A.S., Ng, K.L.R., Pyrka, A., "The archetype sustainable house: investigating its potentials to achieving the net-zero energy status based on the results of a detailed energy audit, in: Proceedings of the 1st International High Performance Buildings Conference", 2010. p. 1-8.

5.43. Safa, A., Fung, A.S, Kumar, R., "Performance of two-stage variable capacity air source heat pump: Field performance results and TRNSYS simulation", Energy and Buildings, 2015.94: p. 80-90.

5.44. Kumar M, Kar IN, "Non-linear HVAC computations using least square support vector machines", Energy Convers Manag, 2009.50: p. 1411-8.

5.45. Zhang, J., Fung, A.S, "Experimental study and analysis of an energy recovery ventilator and the impacts of defrost cycle", Energy and Buildings, 2015.87: p. 265-271.

5.46. Rupayan, B., "Assessment and energy benchmarking for two archetype sustainable houses through comprehensive long term monitoring", 2010, Master of Applied Science, Ryerson University.

5.47. Kamel, R., Fung, A.S., "Modeling, simulation and feasibility analysis of residential BIPV/T+ASHP system in cold climate—Canada", Energy and Buildings, 2014.82: p. 758770. 
5.48. VIESMANN, Technical Data Manual, Gas-fired wall-mounted condensing boiler 12 to $285 \mathrm{MBH}$ ( 3.5 to $83.5 \mathrm{~kW}$ ), Vitodens 200-W Series B2HA.

5.49. Abedi, S. Alimardani, A., Gharehpetian, G., Riahy G., Hosseinian, S., "A comprehensive method for optimal power management and design of hybrid RES-based autonomous energy systems", Renewable and Sustainable Energy Reviews, 2012.16: p. 1577-1587.

5.50. Lam, K., Lai, T., Lo, W., To. W., "The application of dynamic modelling techniques to the grid-connected PV (photovoltaic) systems", Energy, 2012.46: p. 264-274.

5.51. Yu, D., "Power Management of a Grid-Connected Distributed Energy Resource System," 2014, Toronto, Canada, Ryerson University.

5.52. Jansuya, P., Kumsuwan, Y., "Design of MATLAB/simulink modeling of fixed-pitch angle wind turbine simulator," Energy Procedia, 2013.34: p.362 - 370.

5.53. Chung, D., Davidson, C., Fu, R., Ardani, K., Margolis, R., "U.S. Photovoltaic Prices and Cost Breakdowns: Q1 2015 Benchmarks for Residential, Commercial, and Utility-Scale Systems, 2015, U.S. Department of Energy, National Renewable Energy Laboratory.

5.54. Cost of electricity by source, 2016, Wikipedia, https://en.wikipedia.org/wiki/Cost_of_electricity_by_source (July $3^{\text {rd }}$ 2016). 